\title{
Advanced Driver Fatigue Research
}




\section{Foreword}

This report summarizes the findings of the Advanced Driver Fatigue Research project conducted by the Center for Intelligent Systems Research (CISR) of the George Washington University (GWU), and funded by the Federal Motor Carrier Safety Administration (FMCSA) of the U.S. Department of Transportation (USDOT). The goal of this project was to develop an unobtrusive drowsy driver detection system for commercial motor vehicles. CISR previously developed an effective drowsiness detection system for automobiles that relied solely on the driver's steering patterns for input. This project's evaluation of this system confirms that this approach is equally valid for truck drivers, despite the differences between truck and automobile driving. The drowsy driver detection system exhibited acceptable false positive and false negative readings and an ideal warning rate before crashes.

Although the report can be helpful to the general public in understanding fatigue in commercial trucking operations, the report is primarily targeted toward commercial motor carriers and drivers.

This publication is considered a final report and does not supersede another publication.

\section{Notice}

This document is disseminated under the sponsorship of the Department of Transportation in the interest of information exchange. The United States Government assumes no liability for its contents or use thereof.

This report does not constitute a standard, specification, or regulation.

The United States Government does not endorse products or manufacturers. Trade or manufacturers' names appear herein only because they are considered essential to the object of this document. 


\section{Technical Report Documentation Page (Form 1700.7)}

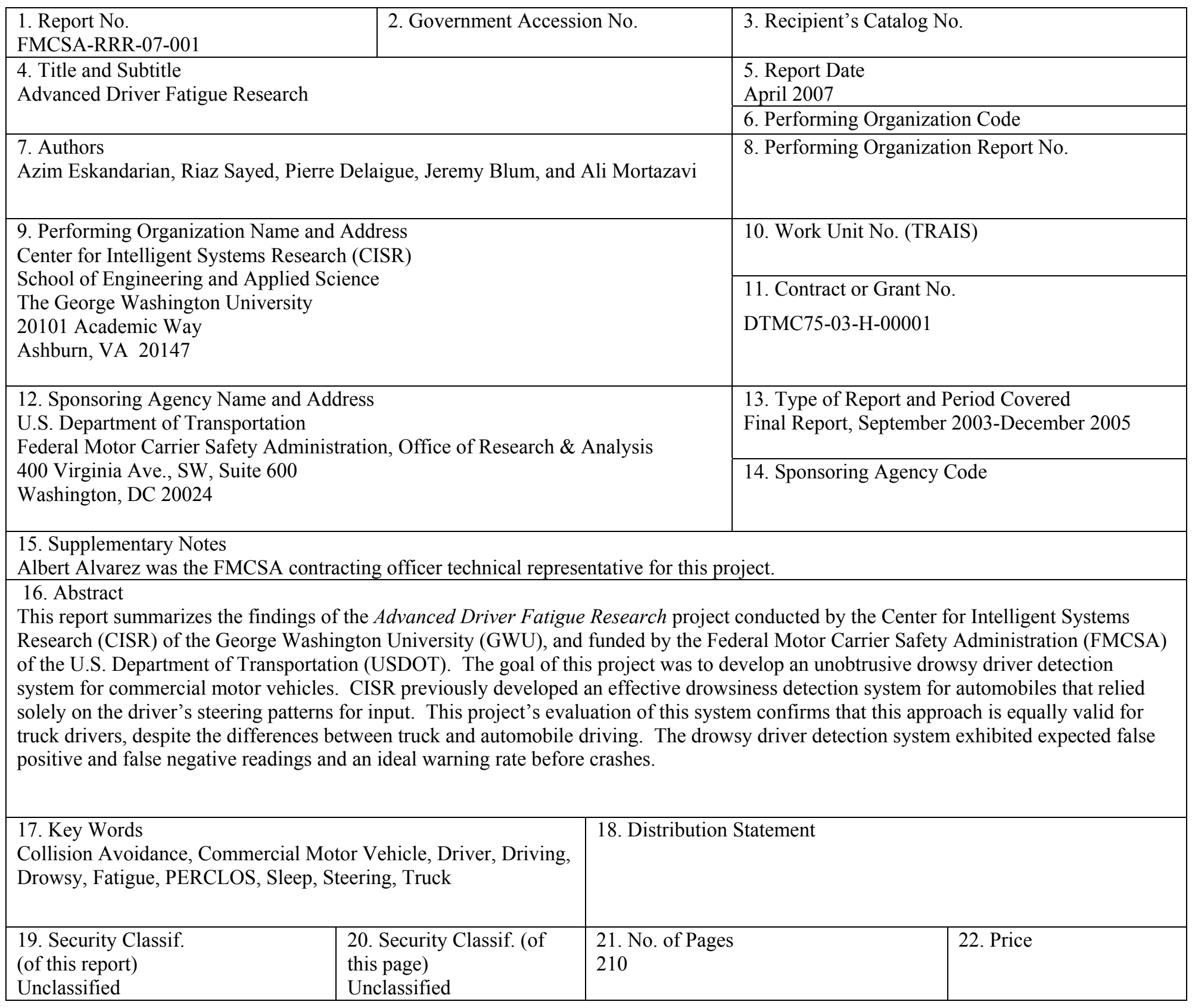




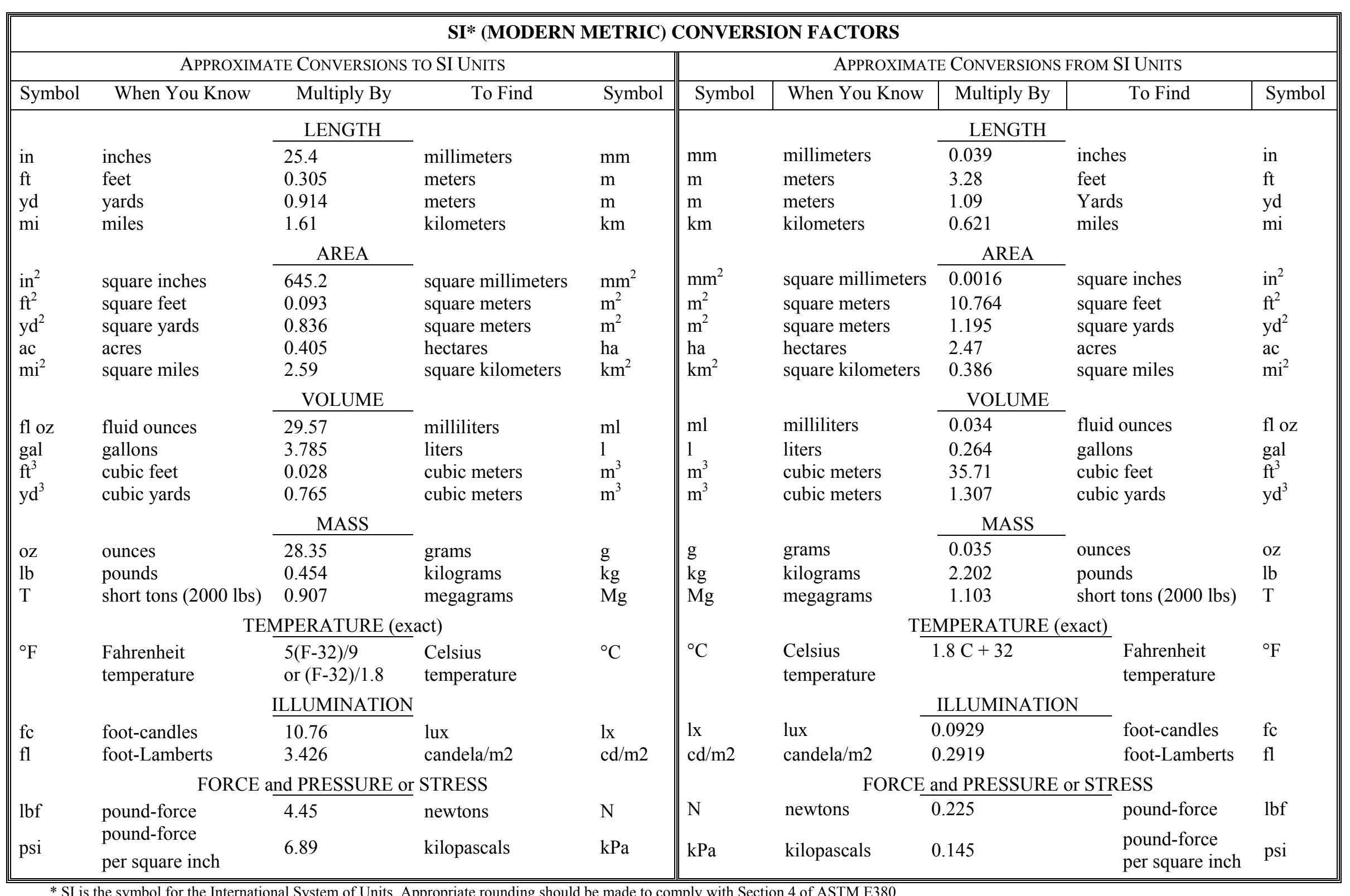

\footnotetext{
* SI is the symbol for the International System of Units. Appropriate rounding should be made to comply with Section 4 of ASTM E380.
} 


\section{TABLE OF CONTENTS}

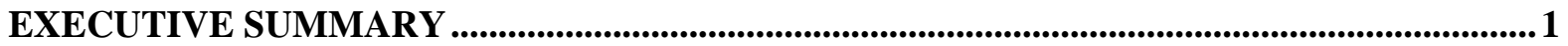

1. INTRODUCTION......................................................................... 2

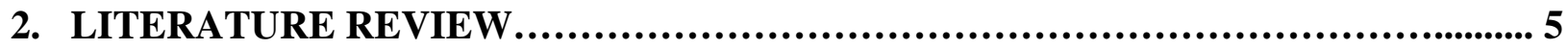

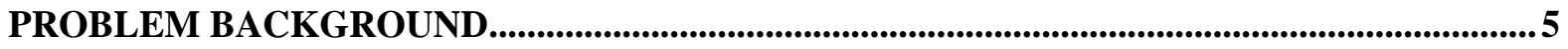

FACTORS CONTRIBUTING TO FATIGUE RELATED ACCIDENTS.....................................6

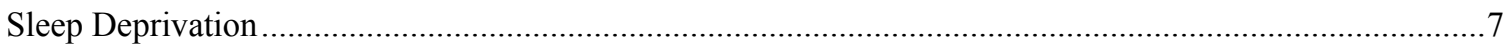

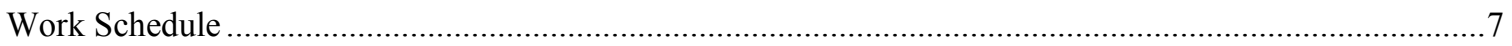

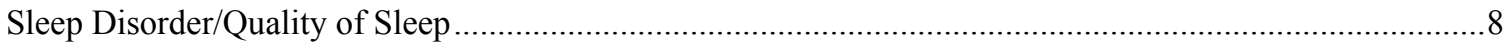

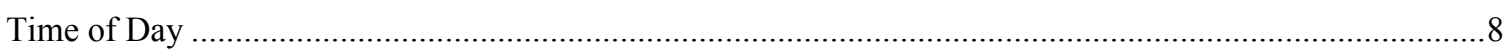

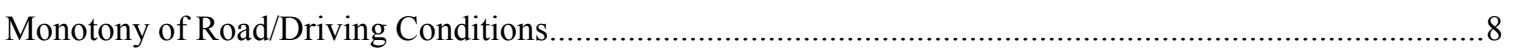

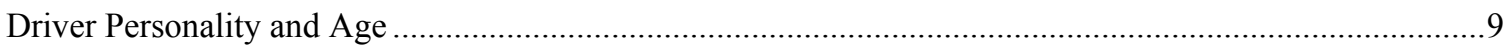

CHARACTERISTICS OF DROWSINESS/FATIGUE RELATED ACCIDENTS .......................9

EARLY ATTEMPTS AT DETECTING DRIVER DROWSINESS ............................................10

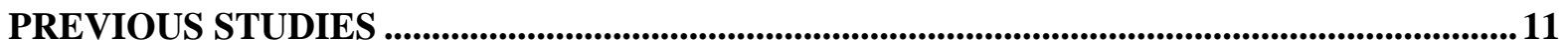

SENSING DRIVER'S PHYSICAL AND PHYSIOLOGICAL PHENOMENON ........................ 11

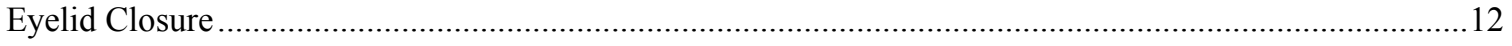

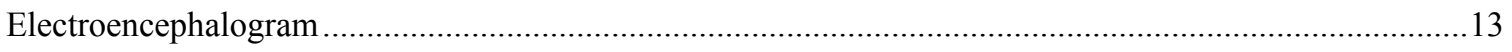

SUITABILITY OF VEHICLE PERFORMANCE OUTPUT FOR DROWSINESS

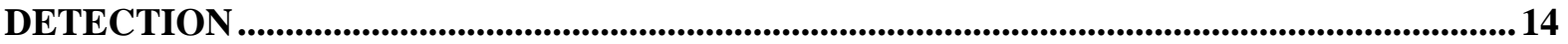

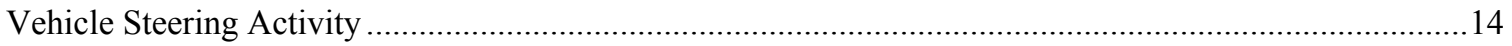

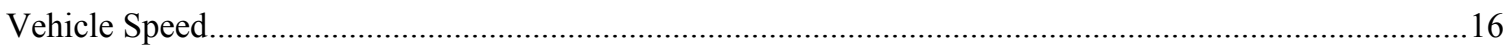

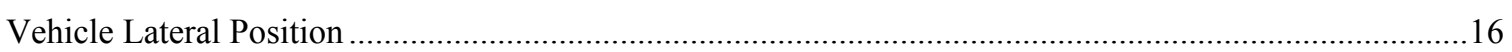

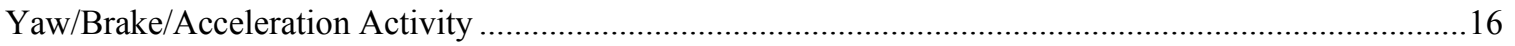

Summary of Suitability of Vehicle Performance Output for Drowsiness Detection .....................................17

3. TRUCK DRIVING SIMULATOR LABORATORY $\ldots \ldots \ldots \ldots \ldots \ldots \ldots \ldots \ldots \ldots \ldots \ldots \ldots . . \ldots 18$

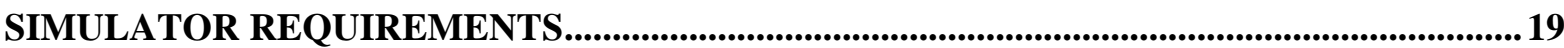

Review of Existing Simulators / Performance Objectives of the Simulator....................................................19

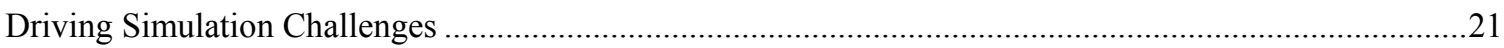

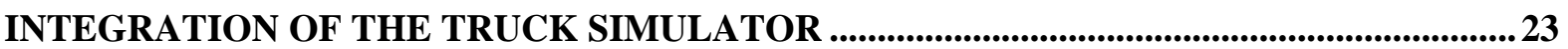

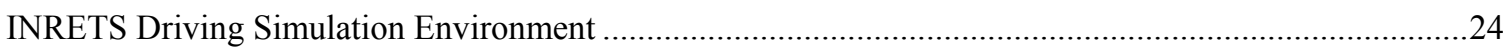

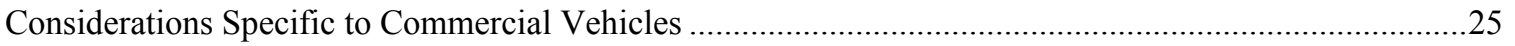

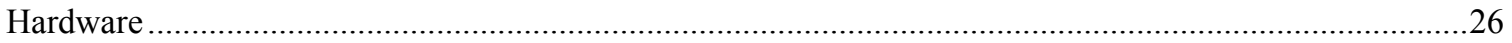

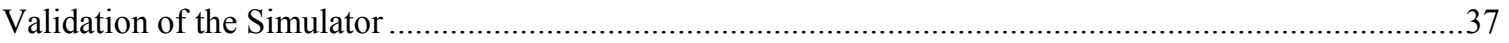


4. EXPERIMENT AND DATA COLLECTION.......................................40

OVERVIEW OF THE DROWSY DRIVING EXPERIMENT.........................................................40

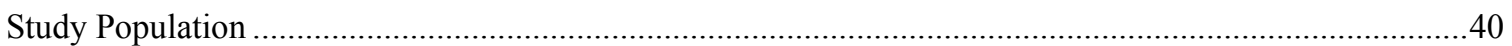

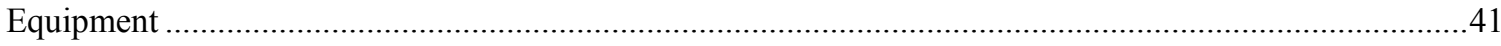

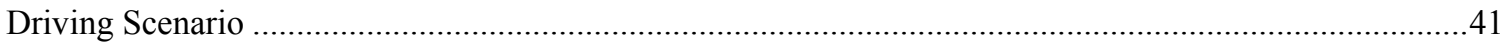

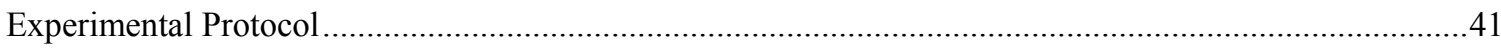

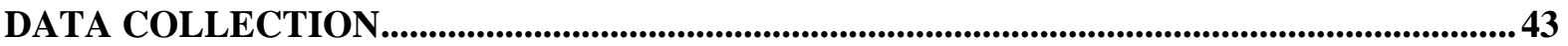

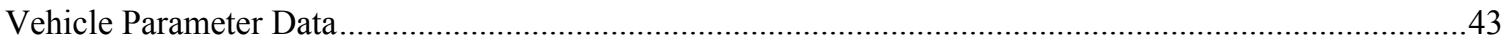

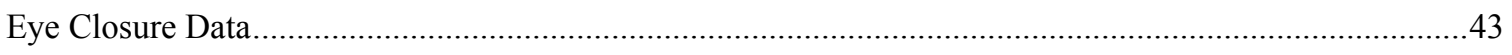

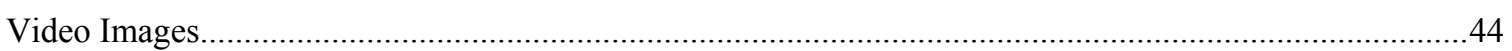

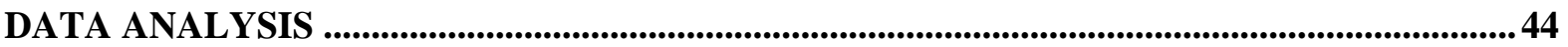

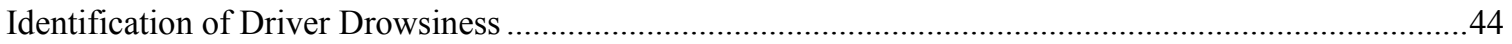

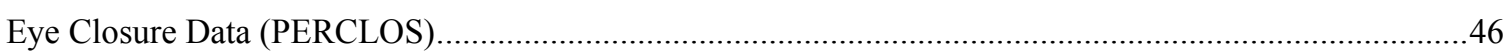

Correlation of Performance Variables with Drowsiness Development ........................................................47

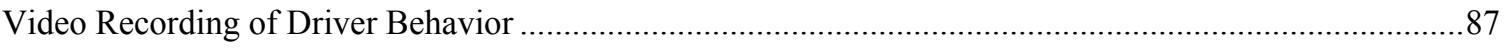

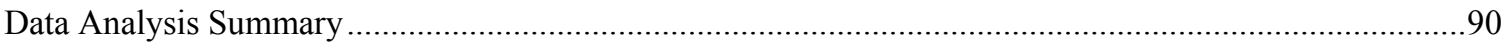

5. DEVELOPMENT OF ALGORITHMS FOR DRIVER DROWSINESS DETECTION

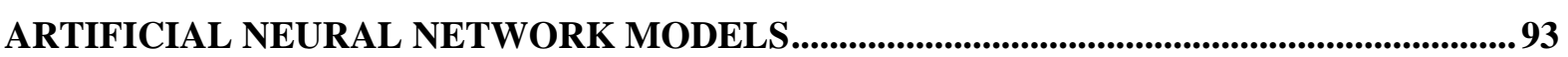

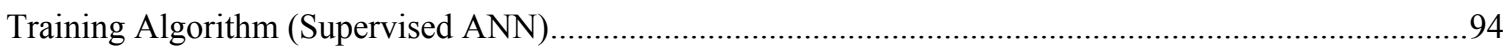

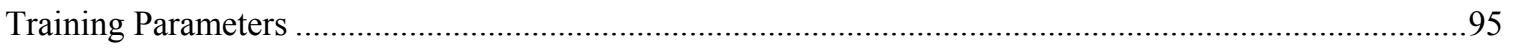

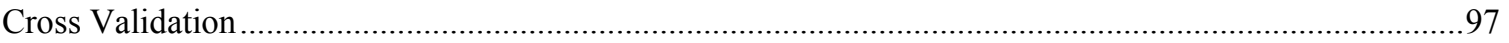

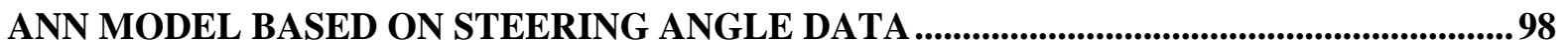

DATA PREPROCESSING AND INPUT DISCRETIZATION (STEERING ANGLE) ..............98

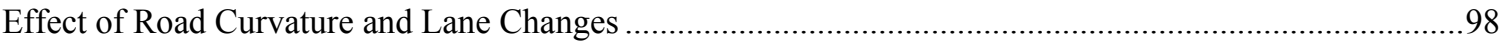

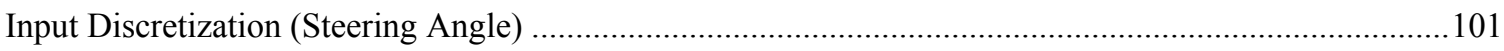

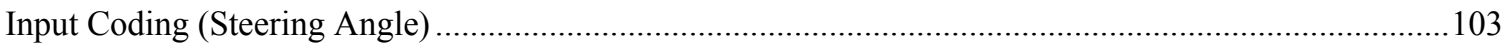

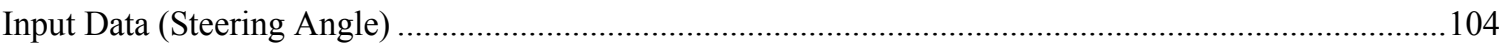

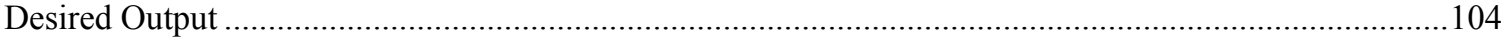

ANN ARCHITECTURE (STEERING ANGLE) ............................................................................ 105

TRAINING DATA SET (STEERING ANGLE) ............................................................................ 106

ANN TRAINING (STEERING ANGLE) ….............................................................................. 106

ANN TESTING (STEERING ANGLE) ......................................................................................... 108

ANN MODEL BASED ON STEERING AND EYE DATA ......................................................... 108

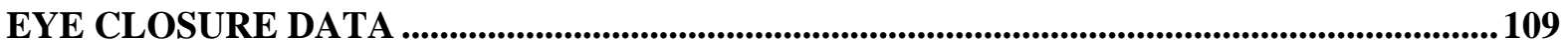

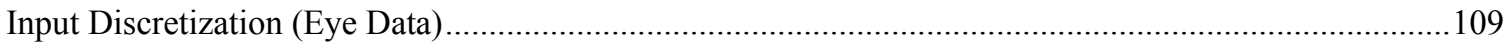

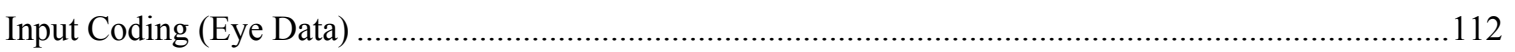

Input Data (Steering + Eye) ......................................................................................................... 112

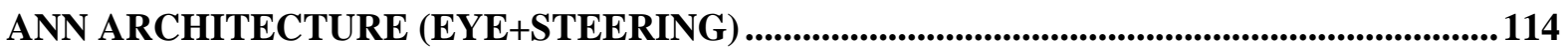




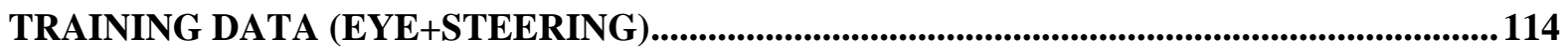

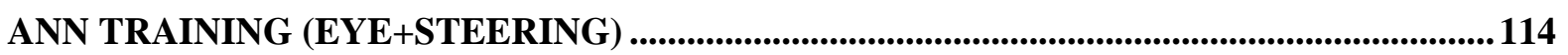

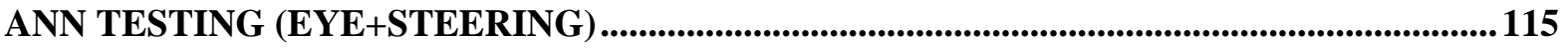

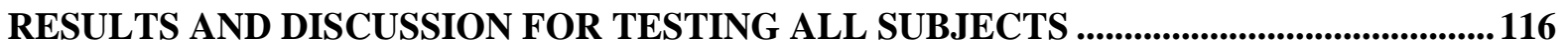

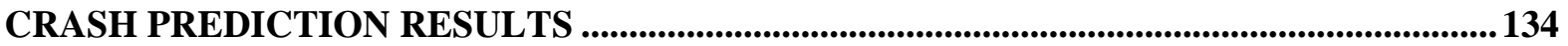

SUMMARY AND COMPARISON WITH PREVIOUS DATA ............................................. 134

6. DRIVER WARNING...............................................................137

WARNING SYSTEMS CHALLENGES ................................................................................138

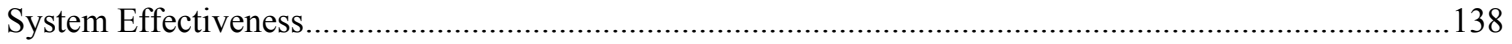

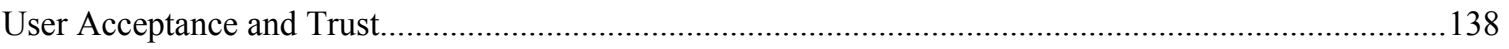

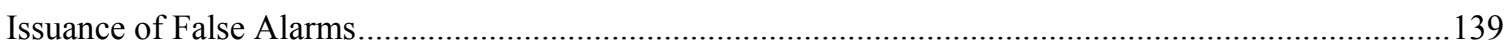

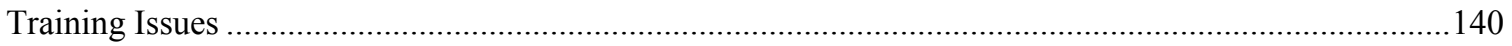

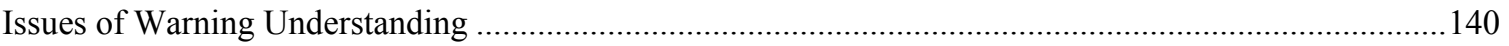

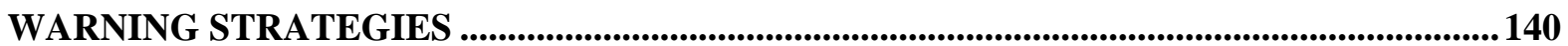

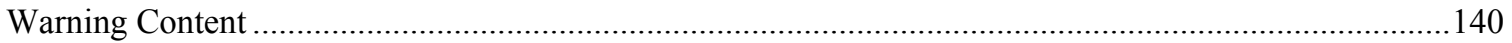

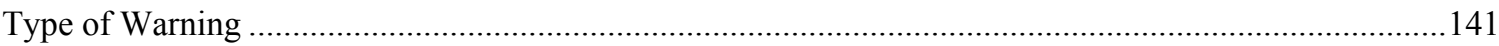

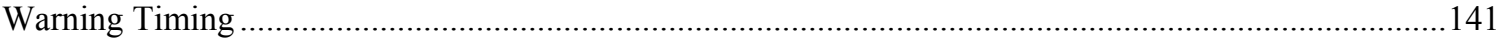

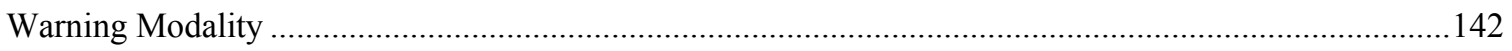

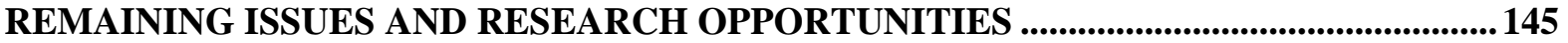

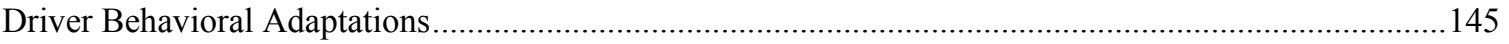

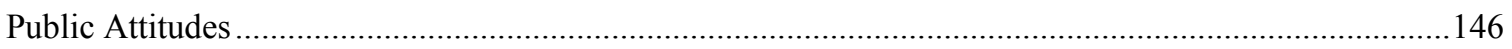

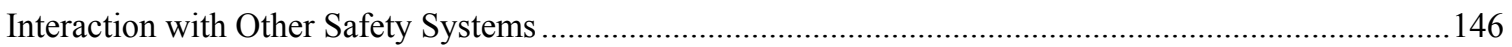

Evaluation of a Driver Drowsiness Warning System in a Driving Simulator ...........................................146

7. CONCLUSIONS AND FUTURE WORK...............................................147

APPENDIX A. RESULTS FROM PREVIOUS ANN MODELS............................150

APPENDIX B. DESIGN, FABRICATION, AND ADAPTATION OF HARDWARE

ELEMENTS FOR CISR TRUCK SIMULATOR.....................................162

LIST OF REFERENCES............................................................. 


\section{EXECUTIVE SUMMARY}

This report summarizes the findings of the Advanced Driver Fatigue Research project conducted by the Center for Intelligent Systems Research (CISR) of the George Washington University (GWU), and funded by the Federal Motor Carrier Safety Administration (FMCSA) of the U.S. Department of Transportation (USDOT). The goal of this project was to develop an unobtrusive drowsy driver detection system for commercial motor vehicles. CISR previously developed an effective drowsiness detection system for automobiles that relied solely on the driver's steering patterns for input. This project's evaluation of this system confirms that this approach is equally valid for truck drivers, despite the differences between truck and automobile driving. The drowsy driver detection system exhibited acceptable false positive and false negative readings and an ideal warning rate before crashes.

A system that relies solely on steering inputs provides a number of benefits over the more common means of detecting drowsiness through eye-tracking. A steering-only detection system is unobtrusive, capable of being implemented inexpensively with a minimal amount of additional sensors and computing power, and immune to problems associated with eye-tracking systems such as performance degradation under low-light conditions or when drivers wear glasses. A steering-only system is based on the hypothesis that people steer differently when they are drowsy. Drowsy driving is marked by a lower vigilance in lane keeping which leads to fewer micro-steering corrections and more macro-steering corrections. Given the variability in driving styles and human behavior, a precise model of fatigued steering behavior is extremely difficult to develop. However, in previous studies, CISR has successfully used Artificial Neural Networks (ANN) to successfully learn patterns of fatigued and non-drowsy steering.

After conducting a literature review of previous research and studies, CISR developed a full-size, fixed-base truck driving simulator laboratory, in partnership with the French National Institute for Transport and Safety Research (INRETS). Experiments were then conducted using fourteen drivers, with both truck driving experience and valid commercial driver's licenses, who participated in the experiment which consisted of three driving sessions for each subject. Each driver participated in a practice session, and then a morning session followed by a late-night driving session. During this night session, most of the subjects experienced one or more episodes of drowsiness.

Two ANN systems were developed to detect driver drowsiness. One of the systems included inputs of both eye closure and steering activity, while the other ANN used only steering activity. Both the steering-with-eye-tracking ANN and the steering-only ANN performed well, at levels

analogous to the previous passenger vehicle detection systems. The steering-only system had an accuracy of $85 \%$ in predicting drowsy intervals with a false alarm rate of $14 \%$. Moreover, it issued a timely warning in $100 \%$ of the collisions that were studied in this project.

The last component of this project involved research into viable drowsy driver detection warning systems. A review of existing literature into drowsy driver warning systems and ITS warning systems, in general, indicated the development of the design goals for a proposed alarm system. The timing, intensity, and nature of the warning system to be integrated as part of the study with the detection algorithm are discussed in this report. 


\section{INTRODUCTION}

This report is submitted by the Center for Intelligent Systems Research (CISR) of the School of Engineering and Applied Science (SEAS) of the George Washington University (GWU) to the Federal Motor Carrier Safety Administration (FMCSA) of the U.S. Department of

Transportation (USDOT). This is the final document and the last of the series of reports that were submitted throughout the duration of the project.

The goal of this project was to develop a drowsy driver detection system for commercial motor vehicles. The goal of this project was met through two specific objectives. The first objective of this study was to arrive at an experimentally validated system for fatigue detection in commercial motor carriers. By monitoring the driver's steering activity and eye closure behavior, the aim for this system is to be able to detect a state of driver drowsiness or fatigue sufficiently early to take preventive measures against potential crashes. The emphasis in this project was on development of an algorithm for an unobtrusive monitoring system which mitigates the need for eye tracking as much as possible. A second objective was to research viable countermeasures, for example, warning systems that minimize the severity of crashes and reduce injuries and fatalities in highway crashes involving trucks.

In previous studies, CISR developed an unobtrusive drowsiness detection system that performed extremely well in experiments with car simulators. This system monitored steering activity to assess a driver's level of alertness. The underlying hypothesis for this system is that driver's steering activity is affected by drowsiness. A drowsy driver will tend to make fewer microsteering corrections and compensate with more macro-steering corrections. The variability in driving behavior both for one person at different times and across multiple drivers precluded the development of a precise analytical, model of steering behavior. However, an Artificial Neural Network (ANN) system was able to learn the relationship between drowsiness and steering behavior. In previous experiments, this system detected the onset of drowsiness for car drivers with a very high degree of accuracy.

However, it was not clear whether this system would also work for drowsiness detection in large commercial vehicles. Significant differences include the additional experience and training of truck drivers, the dynamics of the trucks versus cars, and the different feel of the steering systems in trucks and cars. Consequently, altered driver behavior and steering signals might limit the effectiveness of this approach for tractor trailers.

These differences notwithstanding, this project demonstrated that this approach is equally applicable for the detection of drowsiness in truck drivers. The effectiveness of the system for truck drivers was tested in simulator-based experiments. A truck driving simulator was constructed for the purpose of this project. Designed to produce the realism required for the drowsy driver experiments, this simulator included a full-size cab, 135 degree forward field of view, projected scenes for both side mirrors, force feedback steering wheel, a gearbox, and realistic action for the accelerator, brake, and clutch pedals. The simulated roadway was based on I-70 in the state of Kansas. The simulator includes extensive data acquisition equipment, including instrumentation of all of the controls in the cabin, driver eye tracking, and video 
recording of the subjects from multiple camera angles. Before the experiments were conducted, the simulator was validated for realism by both truck drivers and experts in truck simulators.

Fourteen drivers, with both truck driving experience and valid commercial driver's licenses, participated in the experiment which consisted of three driving sessions for each subject. First, the subjects participated in a practice session, which lasted approximately one hour, in which they became accustomed to driving the simulator. Sometime later, usually the next day, the subject would then participate in a one-hour morning session, which was held between 8:30 and 9:30 am. The purpose of the morning session was to establish a baseline of predominantly nondrowsy steering behavior for each subject. During the day, the subject was instructed to avoid napping and excessive caffeine consumption. That night, a member of the research team would drive the subject from their abode to the simulator. The subject would then participate in a night driving session starting around 1:30 am, with each session lasting for approximately two hours. During this night session, most of the subjects experienced one or more episodes of drowsiness.

The data generated from these experiments was analyzed thoroughly to evaluate inputs for a drowsy driver detection system and performance metrics for the system. This analysis showed a correlation between high amplitude steering corrections and drowsiness. This analysis also indicated that the crashes are strongly correlated with drowsiness. Due both to this correlation and the primary purpose of the drowsy driver warning systems, collision avoidance, the ability of the drowsiness detection system to detect drowsiness in a timely fashion prior to a collision was established as the most important evaluation criterion.

Two ANN systems were developed to detect driver drowsiness. One of the systems included inputs of both eye closure and steering activity, while the other ANN used only steering activity. A small portion of the data from the morning and night sessions was extracted for training and cross validation of these ANNs. The performance of the systems was then evaluated with the remaining data. A key component of both ANNs is the data preprocessing that discretizes the steering signal into a format that is designed to produce a generalized signal that removes the variations in steering strategies across different drivers and different driving platforms.

Both the steering-with-eye-tracking ANN and the steering-only ANN performed well at levels analogous to the previous passenger vehicle detection systems. The steering-with-eye-tracking ANN had an accuracy of $88 \%$ with a false alarm rate of $9 \%$. The steering-only ANN had an accuracy of $85 \%$ with a false alarm rate of $14 \%$.

The steering-only ANN performed extremely well in the most important evaluation criterion, the ability to timely detect and warn drivers before a collision due to drowsy drivers. Although all the crashes were examined, the first two crashes for each subject are the most relevant, because the driver may remain drowsy through multiple later crashes. The system issued a warning in a timely fashion for $100 \%$ of the first two crashes experienced by any of the subjects. On average, 5.6 warnings were issued during the 5 minutes preceding the crash, with the first warning occurring on average 3 minutes and 56 seconds prior to the crash.

Given the success of this system in the simulator experiments, this drowsy driver detection system is ready for development as a prototype system with sensors and computing resources needed for track tests and field operational tests. Since this approach was relatively successful in 
three different sets of simulators with three different simulators, this approach to drowsy driver detection should translate well to real driving conditions.

Chapter 2 of this report consists of a comprehensive review of previous literature and existing systems for the purpose of incorporating the relevant previous findings, eliminating duplication of efforts, and examining the earlier proposed detection and warning systems, with a particular emphasis on truck driving conditions. Factors that contribute to fatigue/drowsiness, measures to counter driver fatigue/drowsiness, previous studies, and surveys are discussed in detail. This chapter corresponds to TASK-1 of this study.

Chapter 3 describes the development of a truck driving simulator laboratory at the CISR, which was used to conduct the experiments for this project. CISR, in agreement with FMCSA, decided to set up a new low-cost truck driving simulator in partnership with the Modeling, Simulation and Driving Simulators (MSIS) research unit of the French National Institute for Transport and Safety Research (INRETS). This new simulator is dedicated to truck driving simulation tasks. CISR constructed the new truck-driving simulator at the GWU campus in Ashburn, VA. Full details about the capabilities, working, and activities performed during construction and assembling of the simulator are discussed. This chapter corresponds to TASK-3 of this study.

Chapter 4 describes the simulator experiments conducted at the CISR truck driving simulator laboratory. During these experiments, truck drivers drove the truck simulator under different levels of fatigue and sleep deprivation. Data was recorded for parameters related to driver eye closure and driving activity. Data recorded in these experiments was used to develop and validate the detection algorithm.

In this study, an ANN was trained to learn the steering behavior of drivers driving under different levels of sleep deprivation in order to identify drowsy driving behavior. The success of the method depends on a unique data-preprocessing scheme, which enables the use of a single ANN for all drivers by incorporating the variability in human driving behavior. Details of the model development and results of the model performance are discussed in Chapter 5. Chapters 4 and 5 correspond to TASK-4 of this study.

The detection algorithm (or system) analyzes data for a specific time interval and then classifies that interval as wake or drowsy. However, when and how should a system warn a driver about the hazardous situation is another topic for driver/human factor research. If the system waits too long, the driver may not have enough time to react and prevent an accident. At the same time, if the alarm goes off when the likelihood of an accident is very low, the driver may get annoyed and ignore the warning or disconnect the system. Similarly, if the alarm is too low, the driver may not notice it and if it is too intense, it may cause panic. Chapter 6 details the proposed alarm system. The timing, intensity, and nature of the warning system to be integrated with the detection algorithm are discussed. This system builds upon the existing data and knowledge gained through previously reported driver warning studies. This section corresponds to TASK-5 of this study.

Appendix A describes the results of previous experiments, which corresponds to TASK-2 of this study. Appendix B provides details of simulator lab development. A list of references cited in the report is also provided. 


\section{LITERATURE REVIEW}

The main objective of this section is to search and review literature related to the detection of driver fatigue and drowsiness. The specific tasks that were performed to meet this objective include:

- Search all the research performed in the field of driver fatigue/drowsiness

- Review the performance and merits and shortcomings of available fatigue/drowsy detection systems

- Review any specific studies or data pertaining to truck driver fatigue/drowsiness

- Review any DOT or driving simulator data available, through FMCSA or other sources

The literature review is organized by the type of technology and methods used for detecting fatigue/drowsiness. A detailed description on the background of the problem of driver fatigue/drowsiness is provided at the beginning. Statistics on the problem size and scope are provided. Various factors that affect driver alertness and causes of driver fatigue are reviewed.

\section{PROBLEM BACKGROUND}

In a 1991 report, the Office of Crash Avoidance Research (OCAR), part of the National Highway Traffic Safety Administration (NHTSA), identified driver drowsiness as one of the leading causes of single and multiple car accidents. NHTSA estimates that 100,000 crashes annually involve driver fatigue resulting in more then 40,000 injuries. The Fatality Analysis Reporting System (FARS) estimates that 1,544 fatalities are due to driver drowsiness related accidents, each year; however, the actual impact of driver drowsiness is most likely much worse than these statistics would indicate. Often it is very difficult to attribute drowsiness as a cause to an accident due to a dearth of physical evidence that would implicate drowsiness. Indeed, it is widely believed that there are a large number of accidents in which the initial cause of the loss of vehicle control is drowsiness but is reported as something else. Recent research confirms that the problem of drowsy driving is indeed more serious than was earlier perceived.

Researchers at Duke University conducted a survey to study the problem of drowsiness in drivers (Tilley et al, 1973). They distributed 1,500 questionnaires at the Durham, North Carolina Department of Motor Vehicle Administration. Out of the 1,500 drivers, 64\% responded that they had become drowsy at some time while driving. More than $7 \%$ said they had fallen asleep while driving for short periods of time. Out of the $64 \%$ who responded that they had experienced drowsiness while driving, more than $31 \%$ said they were not aware of their condition before they actually became drowsy; $10 \%$ of those who experienced drowsiness said they had been involved in one or more accidents due to 
drowsiness or falling asleep. Another 10\% said they had been in a near accident situation due to drowsiness.

The Kanagawa, Japan Prefectural Police also conducted a survey on driver fatigue (Seko, 1984). According to the findings of this survey, $75 \%$ of the drivers admitted that they had driven while drowsy. Seko also reported about another survey conducted by the Shizuoko Prefectural Police in 1973. According to that report, $9 \%$ of all accidents were caused by drowsiness, and $45 \%$ of all traffic fatalities were due to accidents caused by driver drowsiness. According to Planque et al (1991), fatigue is the cause of $26 \%$ of the fatal accidents on the highways of France.

Some of the major findings of the OCAR 1991 report on crash statistics were:

- According to police reports, there were 72,000 crashes (1.1 percent) annually in which driver drowsiness was cited as the primary cause.

- Annually, driver drowsiness was involved in about 14,000 accidents that cause serious injuries (2.9 percent of all serious injuries).

- Accidents involving driver drowsiness caused 1,550 fatalities (3.4 percent of all fatalities).

- These accidents were under reported and the actual number of accidents involving driver fatigue was greater. The Duke, Japanese, and French surveys indicate that the magnitude of this underreporting may be on the order of 10 .

Fatigue has been estimated to be involved in $2 \%$ to $23 \%$ of all crashes (O'Hanlon 1978, Horne and Reyner, 1995), in $4 \%$ to $25 \%$ of single vehicle crashes (Wang and Knipling, 1994 ), in $10 \%$ to $40 \%$ of crashes on long motorways (Shafer, 1993; Dinges, 1995), and in $15 \%$ of single vehicle fatal truck crashes (Wang and Knipling, 1994). Fatigue has been implicated as the most frequent contributor to crashes in which a truck driver is fatally injured (U.S. National Transportation Safety Board [NTSB], 1990).

Crashes that involve a driver falling asleep are on average very serious in terms of injury severity (Pack et al, 1995) and are more likely to occur in sleep-deprived individuals. Accidents involving driver drowsiness have a high fatality rate because the perception, recognition, and vehicle control abilities of the driver reduces sharply while falling asleep. It has been shown by researchers that subjects can not predict when they will have a serious sleep attack (Wylie et al, 1996; Brown, 1997). Driver drowsiness detection technologies may have the ability to avoid a catastrophic accident by warning the driver of his/her drowsiness.

\section{FACTORS CONTRIBUTING TO FATIGUE RELATED ACCIDENTS}

Researchers have identified many factors that can be attributed to the causes of drowsiness related accidents. Factors that influence driver fatigue/drowsiness include greater daytime sleepiness, less sleep, more difficult schedules, more hours of work, a 
driver's age, driver experience, cumulative sleep debt, the presence of a sleep disorder, and the time of day of the accident (Gander and James, 1999; McCartt et al, 2000).

\section{Sleep Deprivation}

The most common cause of drowsiness is a lack of sleep. The effect of sleep deprivation is cumulative and loosing one or two hours of sleep a night can accumulate to cause serious sleep deprivation overtime (Carskadon and Derment, 1981). Sleep disruptions or fragmented sleep causes a loss of sleep and will result in sleep deprivation (Dinges, 1995). Indeed, the loss of one night of sleep can result in extreme sleepiness (Carskadon, 1993).

Many studies have reported the adverse effect of sleep loss on driving. The findings of these studies include:

- Sleeping less then 4 hours per night severely impairs driving performance (Naitoh, 1992).

- Drivers averaging less then 5 hours of sleep per night increase their risk of being in a sleep related crash nearly five times (Stutts, 2003).

- Loss of sleep increases the tendency of falling asleep and reduces driving performance (Wilkinson et al, 1966; Mitler et al, 1997).

- A 1995 NTSB report identified the duration of the driver's last sleep period, the total sleep obtained during the 24 hours preceding the crash, and fragmented sleep patterns as the most important contributing factors towards a drowsiness related single vehicle large truck crashes.

- According to Sweeney et al (1995), the two most important contributing factors in distinguishing between fatigue and non-fatigue related accidents are the duration of the last sleep period and the amount of sleep in the last 24 hours. The duration of continuous wakefulness, acute sleep loss, and the cumulative sleep debt contribute significantly to fatigue related accidents.

\section{Work Schedule}

Many studies point to a driver's work schedule as having a significant impact on driver fatigue, particularly for commercial vehicle operators:

- Researchers like Lin et al (1994) have reported that the total driving time greatly increase the crash risk.

- According to one experimental study, driving performance among truck drivers start declining after 5 hours of driving for drivers with irregular schedules as compared to 8 hours for drivers with regular schedules (Mackie and Miller, 1978).

- Harris et al (1972) found that the driver risk of being involved in a crash increases after 4 hours of continuous driving. 
- Tractor-trailer drivers who violated the hour of service rule are more likely to fall asleep at the wheel (Braver et al, 1992).

- Truck drivers, who split their 8 hours of required off duty time into two shifts, are at increased risk of being involved in a fatal crash (Hertz, 1988).

- Lavie (1986) argues that truck drivers, who work irregular night shifts and are compelled to sleep during the day time, may not be getting the restorative quality of night time sleep.

- Factors associated with sleep-related crashes include working two or more jobs, working night shifts and working more then 60 hours per week (Stutts, 2003).

\section{Sleep Disorder/Quality of Sleep}

Sleep disorders can also have a significant impact on driver performance:

- Individuals with sleep apnea and other sleep disorders that cause excessive daytime sleepiness are at high risk for accidents (Stoohs et al, 1993; Young et al, 1997).

- Drivers involved in sleep crashes are more likely to report that they often or always had problem falling or staying asleep. They are also more likely to report that the overall quality of their sleep is poor (Stutts, 2003).

\section{Time of Day}

Sleep accidents are more likely to occur during the early morning hours from 2 am to 6 am and to a lesser extent during the afternoon from $2 \mathrm{pm}$ to $4 \mathrm{pm}$. Both Mackie and Miller (1978) and Harris (1972) found that a correlation exists between time of day and level of fatigue. Due to circadian rhythms, people feel sleepiness during the afternoon and evening hours, even among people who are not sleep deprived (Dinges, 1995).

Driver schedules that interfere with natural circadian sleep patterns can disturb sleep. Truck drivers who drive during night time are at higher risk of being involved in a crash (Hertz, 1988; Jovanis et al, 1991).

\section{Monotony of Road/Driving Conditions}

A situation is said to be monotonous when the stimuli remain unchanged or change in a predictable manner (McBain, 1970). The monotony of the roadway geometry and environment has been implicated as a cause of driver drowsiness by a number of studies:

- Due to the monotony of highways, highway night drivers are particularly vulnerable to sleep related accidents (Akerstedt and Kecklund, 1994).

- Sleep related accidents may be more common on long stretches of interstate highways and may account for $40 \%$ of fatal accidents (Shafer, 1993). 
- Driving performance degrades at a faster rate on straight road sections than on curves (Desmond and Mathews, 1998).

- $40 \%$ of sleep related accidents occur on highways (McCartt et al, 1996).

- According to one estimate, $30 \%$ of accidents on rural roads are due to driver drowsiness (Fell, 1994).

- In a self reported driver fatigue/drowsiness study, U.S. drivers were found to be more prone to drowsy driving as compared to Norwegian drivers (Sagberg, 1999). Sagberg argues that the cause may be the geometry and environment of U.S. highways. He further argues that the risk of falling asleep is higher on straight, monotonous roads with low traffic, where boredom is more likely to occur.

- A simulator study suggested that fatigue is likely to occur much earlier when driving in a monotonous, low demanding road environment (Thiffault and Bergeron, 2003a).

\section{Driver Personality and Age}

Research has also found a driver's personality and age have a large influence on the susceptibility to drowsiness:

- Many studies have found that there are large individual differences in the susceptibility to become drowsy while driving (Verwey and Zaidel, 2000; Artaud et al, 1994; Wylie et al, 1996). They all argue that some drivers are more affected by study conditions than others.

- Thiffault and Bergeron (2003b) found that drivers who are more sensation seekers may be more sensitive to road monotony and thus more prone to fatigue related driver errors on monotonous, low demanding roads.

- Campagne et al (2004) compared the performance of three age groups in a driving simulator study and found that deterioration of vigilance is correlated with driving errors for drivers aged 60 and above.

\section{CHARACTERISTICS OF DROWSINESS/FATIGUE RELATED ACCIDENTS}

Horne and Reyner (1995) identified criteria by which sleep related vehicle accidents could be identified. These criteria include vehicle running off the road, no sign of braking, no mechanical defect, good weather, and elimination of speeding. NHTSA reported the following statistics on crashes indirectly related to the driver drowsiness/sleepiness: 
- The highest number of crashes occurs during the period from midnight to early morning. More than $40 \%$ crashes occur between $1 \mathrm{am}$ and $7 \mathrm{am}$. The probability of falling asleep is very high during this time interval.

- About $70 \%$ of crashes occur on rural highways with 55 to $65 \mathrm{mph}$ speed limit. This provides a monotonous and calm atmosphere, which is just right for falling asleep.

- The first events that occur in the accident are:

o $64 \%$ are collisions with fixed objects (trees, guardrail, highway sign, etc.), which is another characteristic of sleepy drivers

o $17 \%$ are collisions with another moving vehicle

o $7 \%$ are rollover

o $6 \%$ are collisions with parked vehicles

Crashes related to driver fatigue were classified by Hamouda and Saccomono (1995) who developed an ANN model for identifying patterns in fatigue related accidents. The ANN used various data available from police accident reports to determine if a crash was caused by driver drowsiness or by other non-drowsiness related factors. Similarities in the conditions of drowsiness related accidents could then be identified. The ANN was trained on data from police reports and the database of the province of Ontario.

\section{EARLY ATTEMPTS AT DETECTING DRIVER DROWSINESS}

Early driver drowsiness research used various methods for detection and warning systems. Some of the mechanical devices that were available in the mid-1960s and early 1970s include the following (Hulbert, 1972):

- The electronic Transistor Safety Alarm was a lightweight plastic device that curled around the driver's ear and buzzed when the driver's head nodded.

- The Button Steering Wheel Alarm plugged into the car and was mounted on the steering wheel. An alarm will sound any time the button was released. The device was deemed very impractical.

- The ALERTMASTER was a pedal positioned on the floor to the left of the clutch pedal. Anytime the pedal pressure was released, the horn would sound.

- The Alert-O-Matic was based on the driver response to a light signal presented every 60 seconds. If the driver response (tapping on the horn) was not adequate, an alarm would be set off. 


\section{PREVIOUS STUDIES}

Previous studies have sought to develop algorithms for the detection of drowsiness in drivers. Many of these studies have used cameras or human observers to monitor eye closure for this purpose.

Dingus et al (1985) performed a study to observe the effects of drowsiness on driver performance. They used data from subjects under rested and sleep deprived conditions and developed an algorithm called PERCLOS, which is a measure of the proportion of time that the eyes of a subject are closed over a certain time period, as judged by a human scorer. They reported that best results could be obtained when the performance variable used is the proportion of time when the driver eyes are 80 to 100 percent closed. Dingus et al used eyelid closures as a definition of drowsiness to analyze different performance variables. They performed a correlation analysis between the measure of eyelid closures and other vehicle and driver related measures and reported that a relatively high correlation exists between eyelid closures and lane position measures.

Wierwille et al (1994) conducted a study to determine what variables and combination of variables could be used for the prediction of driver drowsiness. In this study, subjects drove a driving simulator both under rested and sleep deprived conditions. Numerous driver, vehicle, and secondary task performance measures were collected. The measures used in this study were related to seat movement, steering movements, vehicle lateral position, accelerator pedal movements, heading angle, subsidiary task, EEG, and heart rate. Wierwille used PERCLOS and an average drowsiness rating of three observers for each six-minute time interval as measure for drowsiness. To determine the best predictor of drowsiness, Wierwille developed numerous algorithms using multiple regression and discriminant analysis methods. He concluded that no one model will be sufficient under all circumstances and good accuracy could only be achieved by using a combination of models.

\section{SENSING DRIVER'S PHYSICAL AND PHYSIOLOGICAL PHENOMENON}

In the scientific literature, researchers have approached the problem of driver drowsiness detection by using different techniques. These techniques can be broadly classified into two categories based on sensing of driver physical and physiological phenomena and sensing performance output of the vehicle hardware. Each of these techniques is detailed in this section.

A variety of methods are used to sense the physical and physiological phenomena of drivers. These include techniques such as detection by analyzing changes in brain wave or EEG, eye activity, facial expressions, head nodding, body sagging posture, heart rate, pulse rate, skin electric potential, gripping force on steering wheel, and other changes in body activities. 


\section{Eyelid Closure}

Eyelid closure is one of the most obvious approaches to monitoring driver drowsiness. A number of different techniques are available for obtaining this measure.

Erwin (1976) studied various measures to determine whether they were predictive of sleep and reported eyelid closure as the most reliable predictor of the onset of sleep. Haider and Rohmert (1976) evaluated blink rate, while subjects drove a truck simulator for 4 hours, and reported an increase in the blink rate between 80 to $100 \%$. It seems quite obvious that if a driver eyes are closed, the ability to drive a vehicle will be greatly reduced.

Skipper et al (1984) studied the performance of sleep deprived drivers, who performed a $1 \frac{1 / 2}{2}$ hour driving task. The experimenters used a linear potentiometer to manually track the eyelid movements of the drivers. The researchers concluded that eyelid closures could be used as a measure for detecting drowsiness

Ogawa and Shimotani (1997) analyzed data from a driving simulator experiment. They concluded that long duration blinks of half a second or more corresponds to subjective evaluation of sleepiness. The authors define a degree of alertness $(\alpha)$ as

$$
\alpha=\text { number of long duration blinks/total number of blinks. }
$$

The driver was considered drowsy every time the value of $\alpha$ increased above a specified threshold value.

Different techniques have been used to track the eyelid closures. In the Dingus et al and the Hardee et al studies, experimenters manually track the eyelid movements. By looking at the video image on a monitor, an experimenter used a linear potentiometer to track the position of the eyelids.

Ueno et al (1994) used a method based on the Feret's diameter of the eye to track the eyelid closures. The system analyzes the image of driver face taken by a video camera. After separating the eyes from the rest of the facial features, it defines a rectangular window around the eye on the basis of the Feret's diameter of the eye. The maximum number of black pixels along the vertical axis of the window indicates the degree of eye openness and is used, as a basis for judging whether eyes are open or closed.

Electrooculography (EOG) involves the measuring of eye movements through electrodes attached to the skin surrounding the eye. The detection of the eye movements is satisfactory only when the movements are visually unambiguously definable and isolated. Failure rate is very high for atypical eye movements.

Ogawa and Shimotani (1997) used the angle of inclination of eye corners to track the eyelid closures. This angle is steep when eyes are open and shallow when eyes are closed.

Seki et al (1997) developed a method that uses reflection from the retina (bright pupil) to determine whether the eyes are open or closed. In this method, a charge-coupled device 
(CCD) camera, using an infrared light to illuminate a driver's face, captures the driver's facial image, which is then converted into digital images. The pupils are identified on the basis of their geometric features and relative positions using the binary image. The eyes are considered closed when there is no reflection from the retina.

\section{Electroencephalogram}

The Electroencephalogram (EEG) recorded from the human scalp is the most important physiological indicator of the central nervous system activation and alertness. Many researchers have used this physiological indicator to identify the period of drowsiness.

From a state of fully awake to a state of fully asleep, the EEG varies in frequency bands ranging from 0 to 20 hertz. These frequency bands are classified as follows:

- Delta waves ranging from $0-4$ hertz

- Theta waves ranging from $4-8$ hertz

- Alpha waves ranging from $8-12$ hertz

- Beta waves ranging from $13-20$ hertz

An alert mental state is accompanied by fast frequency beta activity, whereas a sleep state is accompanied by slower theta activity. Alpha activity is associated with relaxed experience during which attention is unfocused, showing drowsiness.

Researchers have proposed various methods to extract features from a segment of the raw EEG. In the time domain, average value, standard deviation, and sum of squares of EEG amplitude are most commonly used. In frequency domain, energy content of each band $(\beta, \alpha, \theta, \delta)$, mean frequency, and center of gravity of the EEG spectrum are commonly used. Other models, such as Auto Regressive Moving Average (ARMA) and power spectrum estimation, are also used by some researchers to extract EEG features.

Torsavall and Akerstedt (1987) measured continuous EEG spectra for 11 train drivers. They reported that lapses of attention were preceded by increase in low frequency EEG activity. The researchers also showed that the driver vigilance tends to diminish rapidly after prolonged driving and can be measured by means of spectral analysis of the EEG.

Richardson et $a$. in the European PROMETHEUS project used EEG in conjunction with other variables to find correlation between drowsiness and EEG. Alkerstedt and Gilberg (1990) and Huang et al (1996) used fluctuations in mean frequency of EEG to detect the state of alertness. Generally, EEG is considered suitable for making accurate and quantitative judgments of alertness levels. Wierwille and Ellsworth (1994) found that trained observers could rate the drowsiness level of drivers based on video images of driver faces. 


\section{SUITABILITY OF VEHICLE PERFORMANCE OUTPUT FOR DROWSINESS DETECTION}

An EEG can provide very good detection accuracy, as it is a direct measure of the activity of the central nervous system. However, the problem with an EEG is that it requires the use of electrodes to be attached to the scalp, which makes it very impractical. Eye closure activity can also provide good detection accuracy but capturing eye image unobtrusively can be challenging under certain conditions. Changes in light conditions, correction glasses, angle of face, and other conditions can seriously affect the performance of image processing systems.

Other approaches for detecting driver drowsiness are based on monitoring driver inputs or vehicle output variables during driving. These methods have the advantage of being non-intrusive to the drivers. In this category, the focus of measurement is not on the condition of driver but it is on the performance output of the vehicle hardware. The vehicle control systems that might be monitored for sensing driving operation include the steering wheel, accelerator, and brake pedal. The vehicle parameters that can be measured include the vehicle speed, acceleration, yaw rate, and lateral displacement. Since these techniques allow non-contact detection of drowsiness, they do not give the driver any feeling of discomfort. On the negative side, they are subject to numerous limitations depending on the vehicle type and driving conditions. Wierwille et al (1992) discussed the performance measures as indicators of driver drowsiness in detail. A summary of these measures is presented in the following sections.

\section{Vehicle Steering Activity}

For many years, experiments have been carried out to determine the physical parameters characterizing driving, which could be correlated with EEG parameters that can predict the driver drowsiness. Vehicle steering activity has been cited by many of these studies.

Hulbert (1972) found that the sleep-deprived drivers have a lower frequency of steering reversals (every time the steering angle crosses zero degrees) than that of rested drivers. Researchers like Mast et al (1996) and Dureman and Boden (1972) have found that there is a deterioration of steering performance with drowsiness.

According to Kahneman (1973), effort and Steering Wheel Reversing Rate (SWRR) are linked. He showed that the SWRR decreases under the influence of substances such as alcohol, which reduces driver activation level. Ryder et al (1981) found that the frequency of steering reversals decreases with time on task.

Yabuta et al (1985) hypothesized that when a driver is drowsy or falling asleep, his/her steering behavior becomes more erratic. Yabuta defined this erratic steering behavior as "more frequent steering maneuvers during wakeful periods, and no steering correction for a prolonged period of time followed by a jerky motion during drowsy periods."

Dingus et al (1985) found that several steering related measures, such as steering velocity, steering wheel increment, and low velocity steering, can be used to predict drowsiness. 
Mackie and Wylie (1991) provided a review of patterns of steering wheel movements and vehicle speed. They have affirmed the complexity of the analysis of these two variables and reported that the environmental factors could highly affect the steering precision.

A study conducted by Chaput et al (1990) suggests that there exists some correlation between micro steering movements and drop in vigilance. During high vigilance (alert) periods, small amplitude steering wheel movements are frequent, but during fatigued periods, large amplitude movements are more visible.

Elling and Sherman (1994) analyzed actual driving data from one hour of continuous driving by professional drivers. They reported that steering wheel reversals and standard deviation of steering wheel angle are two measures that show some potential as drowsiness indicators. They also reported that gap-size (i.e., the angle that the steering wheel must be reversed before being counted as a reversal) has a major influence on the reversal rate. Their gap-size function has a dead-band that disregards any extremely small reversals such as those due to road variations.

Fukuda et al (1995) developed a driver drowsiness detection system at the Toyota Motor Company. The authors used steering adjustment time to estimate drowsiness. Their method consists of the following:

- Steering adjustment intervals are calculated at different speeds for alert conditions (learning). These intervals vary with speed and individual behavior but it follows the same pattern.

- The steering adjustment intervals are normalized at $80 \mathrm{~km} / \mathrm{hr}$. These intervals are constantly calculated. Whenever it reaches a threshold value, the driver is classified as drowsy. The value of drowsiness threshold is not constant but it varies with speed. The driving threshold is calculated by taking the product of the mean value of learned steering adjustment intervals in the normal state and the mean value of most recent steering adjustment intervals. The results show good correlation with EEG.

Siegmund et al (1996) conducted an experiment based on the performance of 17 long haul truck drivers under alert and fatigued conditions on a closed circuit track. They presented a steering based set of weighing functions. These functions are based on steering angle and steering velocity. According to the researchers, these weighing functions are correlated with EEGs and subjective evaluations of drivers. According to their findings, phase plots of steering wheel angle verses steering wheel velocity can be used as an indicator of drowsiness.

Sayed and Eskandarian (2001) developed an algorithm, which is based on the ANN learning of driver steering. They trained an ANN model using data from a driving simulator, driven by human subjects under various levels of sleep deprivation. The model identified drowsy and wake steering behavior, calculated over fixed period of time, with good accuracy. 


\section{Vehicle Speed}

Generally, variability in speed has not shown any significant results that can be used to predict drowsiness. Safford and Rockwell (1967) reported no increase in speed variability during a 24-hour driving experiment.

Riemersama et al (1977) recorded vehicle speed during an 8 hour night driving experiment. They reported an increase in the standard deviation of speed, calculated over 45 minute intervals, after the first 3 hours of driving.

Mackie and O'Hanlon (1977) recorded speed in a 6 hour driving experiment, with a 45 minute pause after 3 hours of driving. The researchers reported a regular increase in the standard deviation of speed from the third driving hour.

\section{Vehicle Lateral Position}

Several researchers such as Mast et al (1996) found that the lane tracking ability decreases as the time on task increases. Skipper et al (1984) found that measures related to vehicle lane position could be used to detect drowsiness. Variables such as the number of lane deviations, the standard deviation of lane position, and the maximum lane deviation are found to be highly correlated with eye closures. According to Dingus et al (1985), the mean square of lane deviation and mean square of high pass lateral position show good potential as drowsiness indicators.

Stein (1995) studied the effect of impairment on driving performance in truck drivers. Using data from a simulator experiment, Stein found that the standard deviation of lane position increases remarkably after the driver gets fatigued at 13 hours of driving. The standard deviation of the heading error also began to increase after 13 hours.

Pilutti and Ulsoy (1995) performed experiments on the driving simulator at the Ford Research Laboratory for detecting driver fatigue. The results, reported by the authors, show that only the standard deviation of lateral position show significant change and correspond well with the PERCLOS model.

\section{Yaw/Brake/Acceleration Activity}

Dingus et al (1985) found that the yaw deviation variance and the mean yaw deviation (calculated over a three minute period) show some promise to be considered as drowsiness indicators.

However, several researchers found no relation between drowsiness and vehicle yaw, brake, or acceleration. Safford and Rockwell (1967) analyzed data from a twenty-four hour driving experiment and reported that the accelerator pedal reversals are correlated with driving time; however, according to the literature review conducted by Dingus et al (1985), there is little evidence of any relation between accelerator activity and time or drowsiness. In addition, researchers such as Brown (1966) found no evidence of any correlation between accelerator and drowsiness. 


\section{Summary of Suitability of Vehicle Performance Output for Drowsiness Detection}

The main advantages and disadvantages associated with using performance outputs of the vehicle to measure driver drowsiness include:

- No electrodes and wires are to be attached to the body of the driver.

- No cameras, monitors, light sources, or other devices are to be aimed at the driver.

- No dependence on the environmental and other road conditions.

- Less computational power is required for processing signals such as steering angle, which makes the online processing of data easily achievable.

- Hardware requirement for capturing signal from vehicle components such as steering, throttle, and gas paddle are much less than that required for an image processing or human body signals. These are often much cheaper and readily available.

- Because of the non-obtrusive nature of these methods, they are more likely to be applicable to drivers.

- Due to variation in the dynamics of different types of vehicles, a universal system that will fit all vehicles is very difficult to achieve. These systems must be tuned in for the type of vehicle in use.

- Accuracy may not be very high as compared to EEG monitors, since EEG monitors are constantly attached to the body and a signal is received even if the car is not in motion. 


\section{TRUCK DRIVING SIMULATOR Laboratory}

CISR drowsiness detection models are based on experimental data recorded in a driving simulator. Initial experiments were performed in a simulated environment representing a passenger car. To gather additional data for this research, a driving simulator capable of simulating a truck-driving environment was needed. Three options were investigated to arrive at a solution for truck simulations:

- Modify the existing vehicle simulator, with software from Systems Technology Inc. (STI), at CISR to accommodate truck driving simulation

- Rent or short-term lease a truck simulator at an existing facility or from another vendor

- Purchase a new low cost driving simulator capable of simulating truck driving

After considering each option, FMCSA and CISR agreed to set up a new low-cost truckdriving simulator. This new simulator is entirely dedicated and specific to truck driving simulation tasks. This provides CISR with more flexibility to develop, integrate, and test new drowsiness detection and warning systems. In addition, the new technologies and state-of-the-art graphics of this new system allow CISR to conduct its studies with a very advanced simulation tool.

To set up this new driving simulator, a partnership was established between CISR and the Modeling, Simulation and Driving Simulators (MSIS) research unit of the French National Institute for Transport and Safety Research (INRETS). This agreement combined INRETS globally acknowledged expertise in the development of driving simulation tools with CISR experience in integrating driving simulators. In this project, INRETS provided CISR with free access to its simulation software, while CISR was responsible for acquiring the truck simulator hardware and integrating the new simulator. CISR established the new truck-driving simulator at the GWU campus in Ashburn, VA.

In a preliminary phase, several driving simulators were visited. Based on the performance objectives for the truck simulator, existing alternatives for integrating the new simulator were identified. Technical challenges and potential issues involved in realistic simulations of driving were investigated to guide the selection of the simulator hardware and layout.

The second phase of this task consisted of integrating the new driving simulator. In the following pages, full details of the organization and capabilities of the INRETS simulation tools are provided. Work and activities performed to set up the new simulator are then extensively described, including design, fabrication, construction, assembling, and adaptation tasks. Considerations specific to the simulation of commercial vehicle operations and particular equipment required for the study of driver drowsiness are reviewed. The method used for creating a driving scenario for the drowsiness detection experiments is also presented, and details on the defined scenario are provided. 
Prior to conducting the drowsiness experiments, the new truck simulator was calibrated and validated. The INRETS vehicle dynamic model was initialized with the vehicle parameters of the real truck. The resulting heavy truck driving simulation was then tested and rated by professional truck drivers and experts from the driving simulation and human factors research fields.

\section{SIMULATOR REQUIREMENTS}

\section{Review of Existing Simulators / Performance Objectives of the Simulator}

CISR explored several options for a truck driving simulator. Cost, hardware requirements, and technical challenges of each solution were evaluated and weighted. Three options were investigated to arrive at a solution for truck driving simulations:

- Modify the existing STI vehicle simulator at CISR to accommodate truck driving simulation.

- Rent or short-term lease a truck simulator at an existing facility or from another vendor.

- Purchase a new low cost driving simulator capable of simulating truck driving.

Each of the above three options has its own set of advantages and disadvantages. Option one, modify the existing STI simulator, has many advantages as CISR already has the required hardware and software. However, further investigation found the following significant drawbacks:

- Poor Graphics: Graphic display of simulated driving scenario and other objects are of poor quality and cannot be enhanced. Texture, color, and modeling details are not up to date and do not provide a realistic virtual environment.

- Hardware Requirements: It was found that the entire computer and other hardware would have to be replaced in order to run the upgraded software. This would offset any advantage gained by the fact that CISR already owns the system.

- Gearbox and Clutch: STI was not able to provide hardware for simulating truck gearbox and clutch operation. The gearbox has to be purchased from another company that would have created compatibility problems. Writing an interface would have made the job more expensive and time consuming.

- Poor Steering Feel: The feeling of steering aligning torque and road grip in the STI system is not very realistic particularly for simulating heavy truck steering. The steering system design is more suited for small vehicles and is not compatible with a large diameter steering wheel. This would have required the complete redesign of the steering system hardware and control algorithm; again, a very expensive and time-consuming option. 
- Rear Projection for Side View Mirrors: In its current configuration, the STI simulation only supports three video channels and can be used for front projection only. Two extra channels are required to accommodate side view mirrors, which are a very important component of truck driving simulation.

- Data Acquisition Reliability: The STI system, although capable of acquiring a large amount of date with a variety of options, keeps data in the computer memory and saves it to the hard drive only after the successful completion of the simulation. This delayed storage can cause a loss of data in the event of a simulation system or operating system crash. This possibility makes it very undesirable for experiments such as fatigue detection, which require very long runs and continuous data acquisition.

The second option of renting or short-term leasing of a truck simulator was also explored but was not considered to be a favorable option due to various reasons. The options of leasing a simulator that can be moved to the CISR premises were very limited. None of the available options have the required capability and demonstrated operational ability to do the job. The options of renting a simulator at an existing facility were also considered. The major problems with this option were logistical issues particularly for the experiment subjects; recruitment of truck drivers from a far off location was a big issue. Cost and availability were also an issue. Facilities such as NADS are too expensive and not available for a long period of time.

CISR, in mutual agreement with FMCSA, decided to set up a new low-cost truck driving simulator rather than upgrading its current STI car-driving simulator. This new simulator is entirely dedicated and specific to truck driving simulation tasks. This option provides CISR with more flexibility to develop, integrate and test new drowsiness detection and warning systems. In addition, the new technologies and state of the art graphics of this system will allow CISR to conduct its studies with an advanced simulation tool.

A comprehensive review of existing technologies for commercial motor vehicle simulation was performed. Experiences of other research centers and organizations from both public and private sectors were reviewed. In addition, the research team attended various conferences and events related to driving simulation to gain knowledge on driving simulators integration and to establish contacts with key players of the driving simulation field. Several driving simulators were also visited ranging from state-of-theart installations of multi-million dollar budgets, such as the Ford Virttex and Daimler Chrysler driving simulators, to simulators operated by academic transportation research institutes, including the University of Michigan GlobalSim DriveSafety simulator and PennState STI/SCANeR truck driving simulator. The functional requirements for the new CISR truck driving simulator were assessed.

In concurrence with FMCSA, a partnership was established between CISR and the Modeling, Simulation and Driving Simulators (MSIS) research unit of the French National Institute for Transport and Safety Research (INRETS) to set up a new generation simulator at the GWU campus in Ashburn, VA. 


\section{Driving Simulation Challenges}

Driving simulation is a complex task that requires the following four basic components, Gruening et al (1998):

- Simulation of the physics of the vehicle and its interaction with road surface

- Simulation of the surrounding environment, including other vehicles

- Integration of informative systems and displays that enable subjects to interpret the state of the vehicle model (e.g., sound rendering, instrument panels, motion base, etc.)

- Integration of control devices (e.g., steering wheel, accelerator pedal, brake pedal, shift lever, clutch pedal, and other vehicle controls)

In the generic architecture of a driving simulator, a computing unit calculates the vehicle dynamics parameters according to drivers' inputs. Results for vehicle position, speed, acceleration, heading, and traffic information are then sent to the visual display system. Commands for engine noise and subsidiary simulation sounds are sent to the sound reproduction system. Control feedback signals are sent to a steering torque controller, a pedal reverse force system, and cabin instrument panel displays (e.g. the speedometer and tachometer).

The next sections combine results from previous research and experience gained through the development of the CISR new truck driving simulator to identify factors that can affect driving in a simulated environment. The understanding of the following challenges and potential issues is fundamental for the successful design and implementation of a driving simulator.

\section{Visual Cues and the Simulator Display}

Previous research has proven the importance of image refresh rate and time delay on driver's performance in driving simulators (Hogema, 1997; Sudarasan et al, 1997). Refresh rates of $60 \mathrm{~Hz}$ and higher are usually considered ideal. Visual delay should be minimized as much as possible and compensation techniques can be implemented if necessary to bring the system response to driver's inputs closer to real world driving. Also, the presence of roadside objects has been shown to improve drivers' ability to estimate their speed and therefore enhance their driving performance (Glaser and Fisher, 1997; Levine and Mourant, 1996).

\section{Audio Cues and Simulator Sound}

Sound rendering plays an important role in the immersion feeling of a driving simulator subject. Wind and engine noise contribute to fatigue in drivers who have logged many hours. Sirens and horns divert their attention from the task at hand and traffic noise affect a driver's state of being and decision-making. In addition, squealing tires are indicators that the vehicle is being pushed towards its handling limits. Spatialized sound 
generation replicating both vehicle and traffic sounds, and vibrations transmitted to the vehicle cabin can significantly enhance the overall realism of a driving simulator.

Attention should be paid to not provide the driver with an excessively loud driving environment. Measures of physiological stress in simulators have proven that excessive levels of vehicle noise inside the cab can create discomfort and negative response from the drivers (Kim et al, 2000).

\section{Vestibular Cues and Vehicle Motion}

When handling a vehicle, drivers base their decisions on visual, auditory, and inertial stimuli. In some situations, linear and rotational accelerations due to vehicle motion can significantly guide the driving strategy.

Previous experiments have shown that speed is underestimated when based on visual cues only, resulting in people driving faster in fixed base driving simulators than in moving base driving simulators (Reymond et al, 2001).

On the other hand, CISR believes that careful analysis is necessary when integrating a moving platform in a driving simulator as potential problems may arise. Inappropriate motion rendering can degrade the driving impression and contribute to simulator sickness.

Moreover, the decision to include motion generation in a driving simulator has to be based on the intended function of the new driving simulator. For example, while vehicle motion feedback may be necessary for training applications, it is not essential for driver drowsiness studies involving continuous highway driving.

\section{Driving Stress}

Because subjects are conscious that they can not be exposed to risk as they usually are on the real road, smaller levels of stress can be experienced in a driving simulator. This should either be taken into account when designing the testing protocol of driving simulator experiments or discussed in the experiments results. To mitigate potential effects of reduced stress on drivers' performance in a simulator, countermeasures can be envisaged to increase subjects' stress levels. For example, researchers can refrain from telling the subjects about the task to be performed, or researchers can increase the driving task workload.

\section{Simulator Sickness}

Simulator sickness can be induced by a discrepancy or delay between visual and motion stimuli perceived by subjects. A conflict between the displayed driving scene and the inertial accelerations sensed by the drivers can be experienced both in fixed base driving simulators and in simulators equipped with moving platforms where inappropriate motion rendering can occur.

Previous research has investigated the effect of various factors on simulator sickness. Low refresh rates of the visual display, large field-of-views (Lin et al, 2002; Lee et al, 1997), excessive vehicle velocity (Mourant and Thattacherry, 2000), and sharp curves in 
the driving scenario (Nilsson, 1993) are factors susceptible to contribute to simulator sickness.

Effects that can potentially contribute to or mitigate simulator sickness have to be carefully investigated in the early stages of a driving simulator setup when designing the display system and defining the driving scenario. For example, a fixed reference in the displayed image (e.g., a fixed background scene), can reduce the motion impression and provide subjects with a sense of stability. This simple alternative can help reduce simulator sickness.

\section{Steering Torque Feedback}

In the virtual environment of a driving simulator, a driver controls the vehicle position via inputs to the steering wheel. The simulator's steering system should be capable of instructing the driver about the amount of steering correction to apply and transmitting the driver's steering inputs to the vehicle dynamics program during simulation. Since steering is the main control device available to the driver in a driving simulator, a driver's performance during an experiment greatly depends on the quality of the steering torque feedback. Previous literature has extensively reported the importance of the response of steering feedback systems on driver steering activity in driving simulators (Espié et al, 2003; Godthelp, 1995; Miyamoto et al, 1991; Liu and Chang, 1995; Howe et al, 1997; Jang, 1996; Fujioka et al, 1999; Chen and Ulsoy, 2002).

\section{Driver-Vehicle Performance in Simulator and Driving Simulator Validation}

A driving simulator can only be a valuable research tool if it has been validated for a selected driving application. However, no standard method exists for validating a driving simulator.

CISR believes that thorough validation of a driving simulator should include both a static evaluation of the simulator components and a subjective assessment (e.g., questionnaires filled by professional drivers or simulation experts) of the simulator ability to accurately reproduce the task being studied. According to Radwan (1998), the most prominent dependent variables used in simulator validation studies are driving speed, lateral position, and steering behavior.

\section{INTEGRATION OF THE TRUCK SIMULATOR}

As mentioned previously, a partnership was established between the CISR and the Modeling, Simulation and Driving Simulators (MSIS) research unit of the French National Institute for Transport and Safety Research (INRETS). This agreement associates INRETS' recognized expertise in the development of driving simulation tools with CISR's experience in integrating driving simulators. In this project, INRETS provided the CISR with free access to its simulation software, while CISR was responsible for integrating the new simulator hardware. 


\section{INRETS Driving Simulation Environment}

This set of simulation tools consists of several modules organized in a common architecture, called ARCHISIM. The modules include:

- The vehicle dynamics model receives inputs from the simulator cabin acquisition card and computes the real-time response of the vehicle in a traffic model. The vehicle parameters of the INRETS passenger car model were adjusted to simulate realistic truck dynamics behavior. The consistency of the modified model for driver drowsiness applications was then verified by experts and professional commercial vehicle drivers as described later in this report.

- The traffic model of ARCHISIM is able to simulate realistic driving situations involving tens of vehicles in complex traffic environments. Unlike most existing traffic simulation tools, the computation of traffic in ARCHISIM is not based on car-following algorithms but on a unique method, which evaluates the space available in front of a vehicle to determine its behavior in the simulated traffic. This approach is believed to better imitate how drivers behave in real life situations and, thus, leads to a more realistic replication of traffic. Figure 3.1 shows the traffic simulation interface of ARCHISIM with detailed information of the driven vehicles. The behavior of other vehicles can be determined autonomously or programmed to fit a specific experiment's needs.

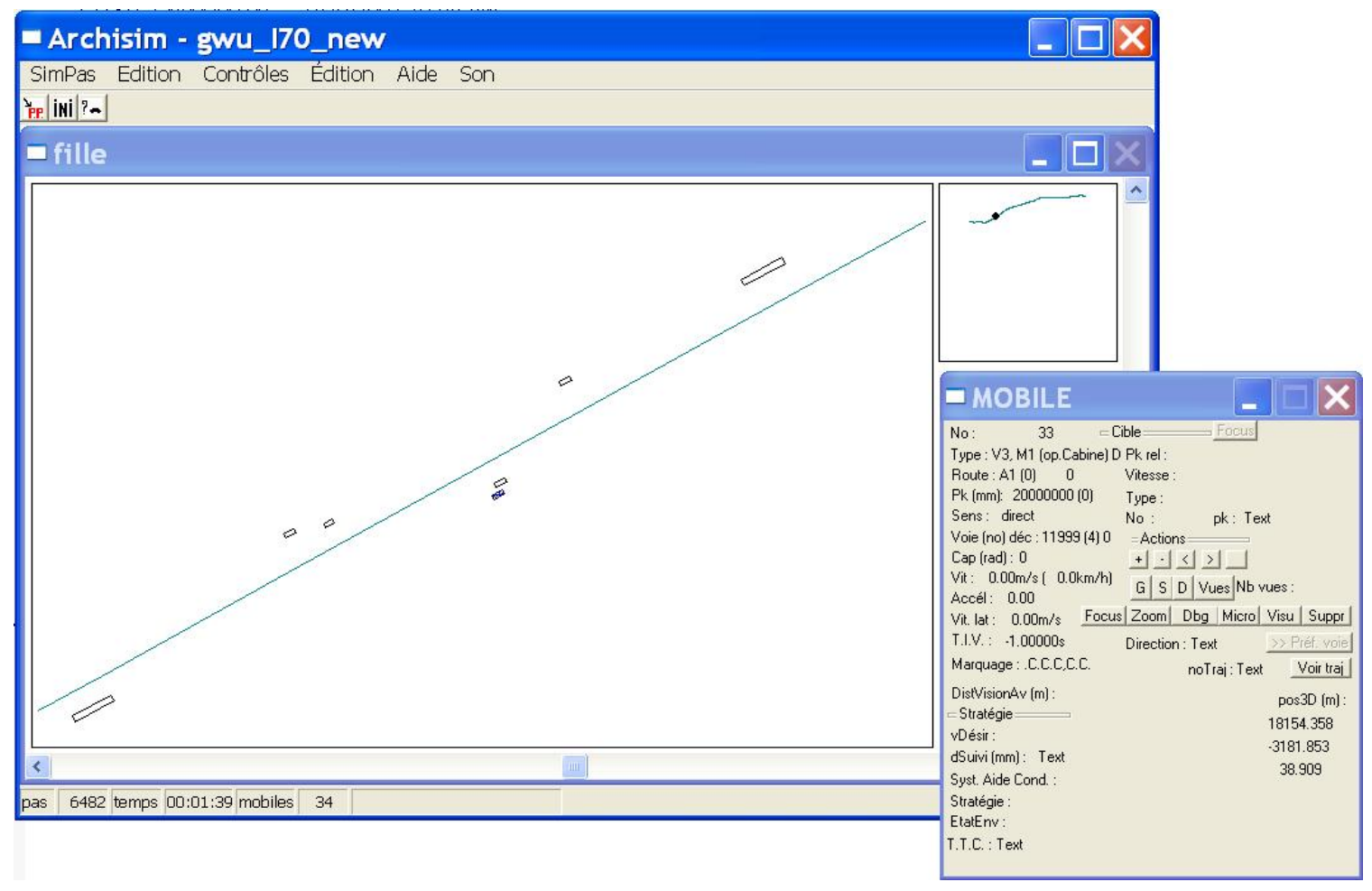

Figure 3.1: Traffic simulation software (Dr2) 
- ARCHISIM enables users to define the sub-components of a driving scenario, the vehicles, traffic signs, roadside objects, surrounding environment, pre-determined traffic events, etc. Actions of the driving scenario can be triggered by the operator during simulation or by various pre-defined traffic conditions, for example, based on distance traveled, time elapsed, vehicle speed, etc.

- INRETS haptic steering feedback algorithm is based on models used in robotic teleoperation (Espié et al, 2003). In this model, the torque generated on the steering shaft is equivalent to the torque generated on the wheel pivot. The induced rolling effect is also taken into account in the computation of the steering torque feedback. More details of the steering feedback calculation method are provided in Appendix B.

- Spatialized sounds from the vehicle engine and surrounding traffic environment are reproduced. Simulated effects include engine sound during vehicle start, vehicle ride, up and down shifting of gears, and the sound of passing vehicles. The car sound samples provided by INRETS were modified for a more realistic truck sound environment. Examples of the changes made to the truck starting sound are shown in Figure 3.2. Their frequency was decreased, their amplitude was increased, and bass sound was added to the original signals to amplify a trembling effect in the cabin equivalent to the normal vibrations in a truck cabin.
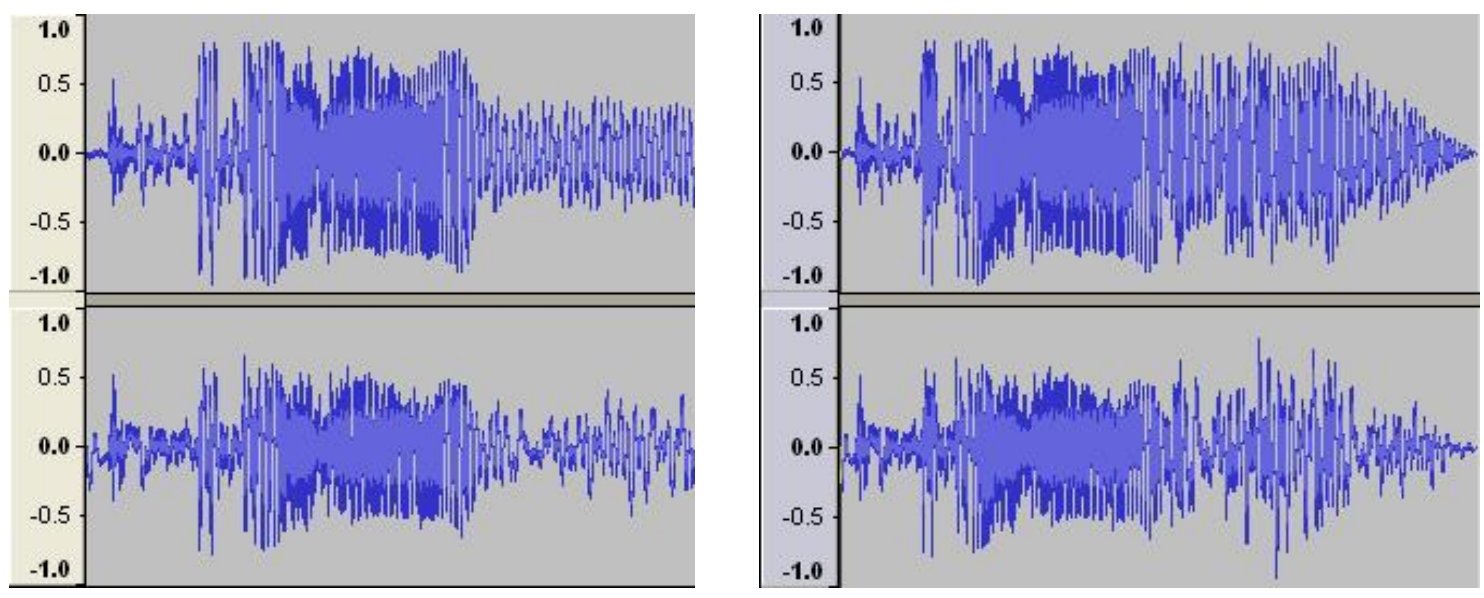

Figure 3.2: INRETS original vehicle start sound and CISR modified truck start sound

\section{Considerations Specific to Commercial Vehicles}

Many aspects of commercial vehicle operation differ from passenger vehicle driving. Features specific to truck driving were identified, and particular issues involved in the duplication of a truck environment were considered, including:

- The height of the truck cabin is critical in the limited space of a laboratory since high cabin roofs can block the images projected on the front screens. This concern guided the selection of the cabin for the new simulator. 
- A hood must be placed in front of the truck cabin to replicate the driving view of most long-nose heavy trucks commonly found on U.S. roads.

- A dominant view of the road ahead should be displayed to reproduce the high driving position of heavy trucks.

- A gearbox must be integrated with both automatic driving and manual driving modes to simulate the different gear shifting modes found in U.S. trucks.

- The sound and vibrations transmitted to the vehicle cabin must reproduce the noisy and trembling environment of a real truck cabin.

- Rearview projection should be available in the simulator cabin to allow drivers to manage the behavior of their trailer as they usually do on the road.

Based on these requirements, the necessary equipment was designed and integrated in the new simulator as detailed in the next section.

\section{Hardware}

In a driving simulator, the driver interacts with a simplified driving environment. Due to physical limitations of the simulator, the driver perceives fewer parameters during simulation than during real world driving. A driving simulator should aim both for replication of an embedded and realistic driving environment and for accurate imitation of the driving task under study. CISR developed its truck driving simulator for driver drowsiness studies according to the organization illustrated in Figure 3.3.

CISR decided not to include a motion base in its new truck simulator. While moving bases can improve the realism of the driving simulation, they are believed to have a limited effect in drowsiness detection experiments, which involve highway driving characterized by low accelerations.

In most low-cost driving simulators, the same computer is responsible for data acquisition from the vehicle cabin; calculation of vehicle dynamics, traffic, and sound models; and generation of force feedback commands for in-vehicle controls. A key point in this simulator is the use of a dedicated micro-controller to manage vehicle cabin interfacing and steering actuator control processes. This additional interface layer, called a Generic Instrumentation Electronic Card (CEGI), receives digital and analog inputs from the vehicle cabin sensors, transmits all measured information to the computing unit of the vehicle dynamics model, controls in-vehicle indicators and dashboard gauges, and sends commands to the steering actuator power module. This second electronic unit then uses Pulse Width Modulation (PWM) to generate steering torque signals to be sent to a DC motor. 


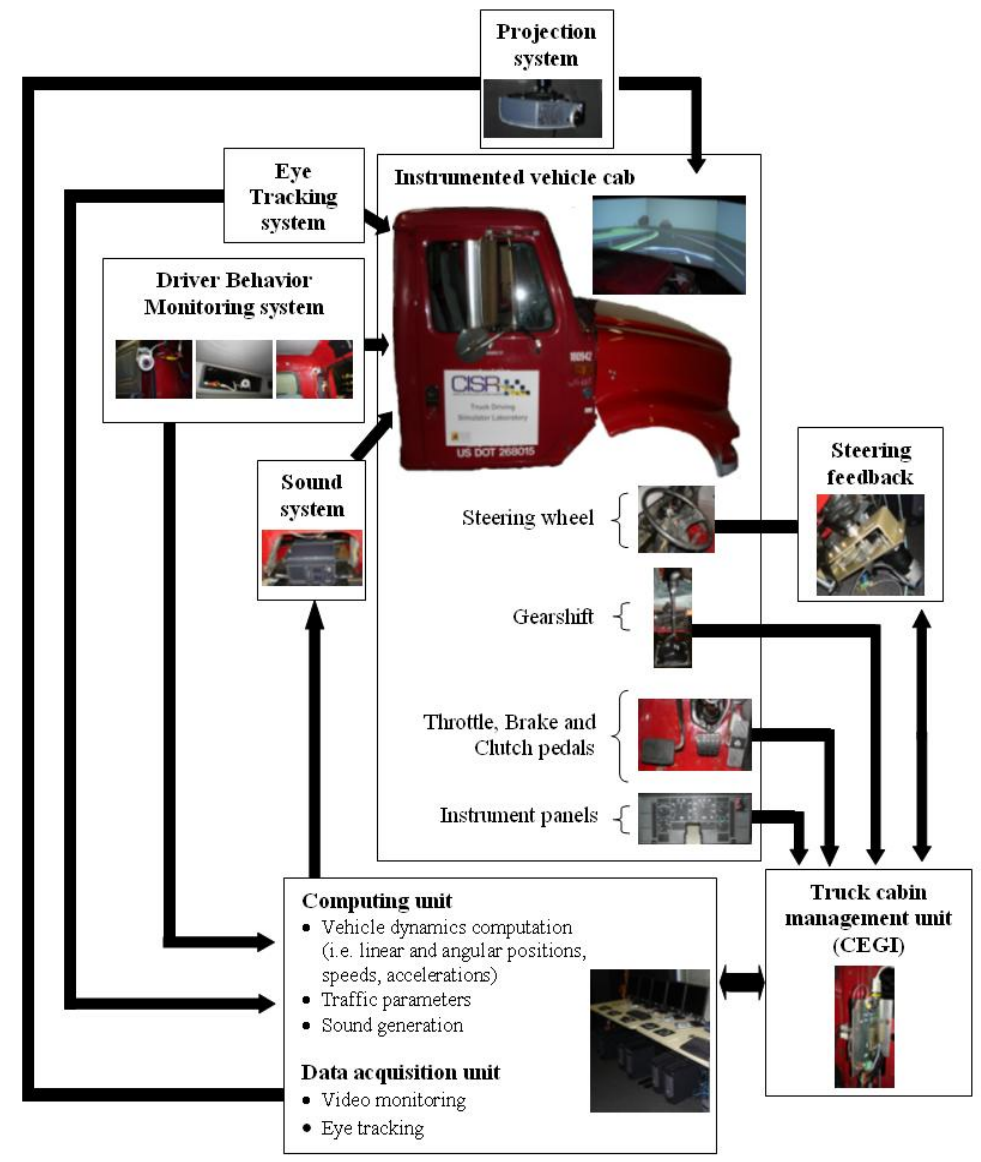

Figure 3.3: A Schematic Diagram of the CISR Truck Driving Simulator

\section{Truck Cabin and Laboratory Layout}

The first task was to prepare and remodel the laboratory to fulfill all requirements of the new driving simulator. The room was entirely painted in black and black shades were installed to enhance the driver's feeling of immersion and improve the contrast of the displayed images. Any objects potentially intersecting with projection beams (including HVAC components, light fixtures, and sprinkler system accessories) were reinstalled to stay clear from the projection path.

The first design challenge in setting up a new driving simulator is to define the layout of the display system and the projectors and screens, around the vehicle cabin in the available laboratory space, as shown in Figure 3.4. The available projecting distance, the width of the displayed scene viewed by the driver, and the lab dimensions guide the selection of vehicle cabin, projectors, and screens, since interference between cabin and projected beams should be avoided. A used International 8100 truck cabin was selected based on its relatively small size and weight in its class (Figure 3.5). Preparation of the cabin and fabrication of a support base are presented in Appendix B. 


\section{Projectors}

The choice of a projector depends on the desired field-of-view (FOV). While a larger FOV produces better immersion of the driving subjects in the virtual environment, it has also been found to contribute to simulator sickness. Compromise values for front projection FOV angles generally range between $110^{\circ}$ and $160^{\circ}$.

Both front and rear projections were required for the truck driving simulator. Three projectors and screens enable a 135-degree wide front vision. The rear vision is created by two projectors and screens, one for each side, which are viewed through the truck side mirrors.

The good contrast ratios, brightness levels, and image sharpness achieved by DLP-based projectors make this technology a good and affordable choice for driving simulation applications.

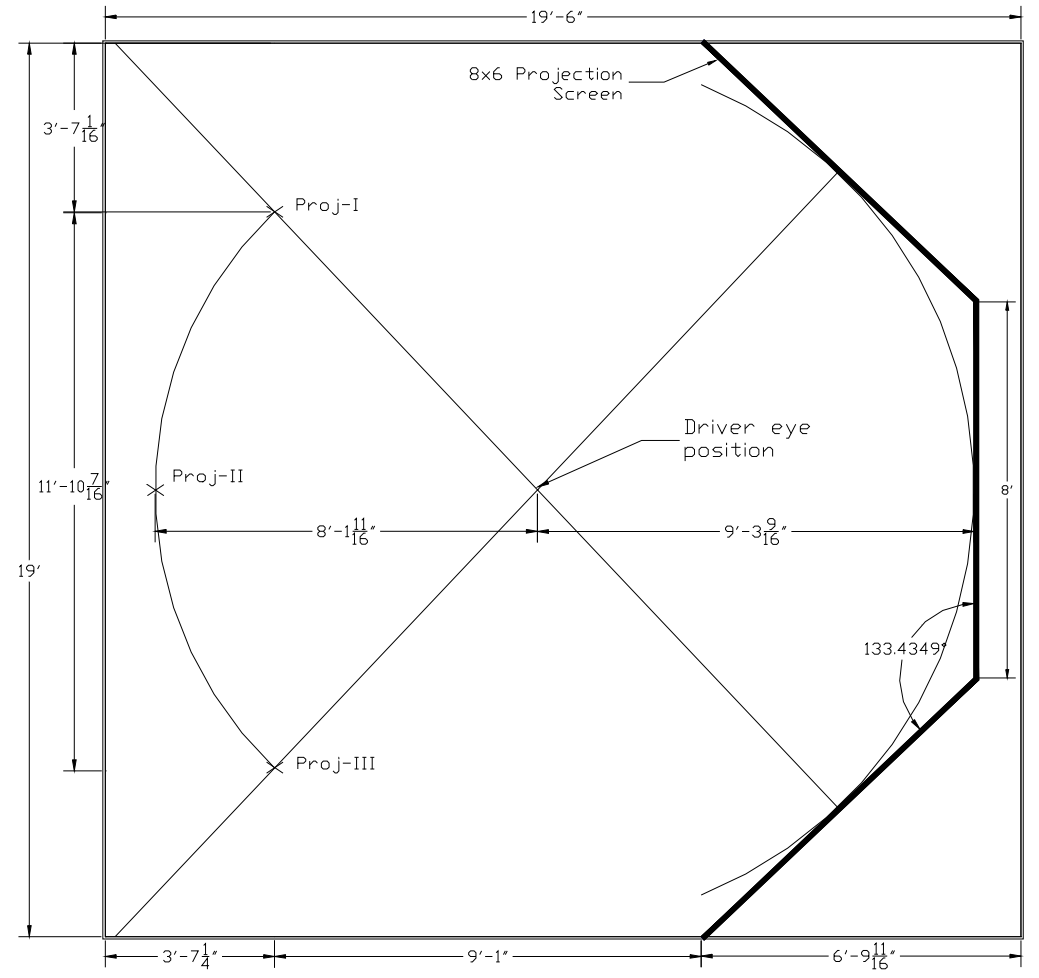

Figure 3.4: General layout of the driving simulator display system 


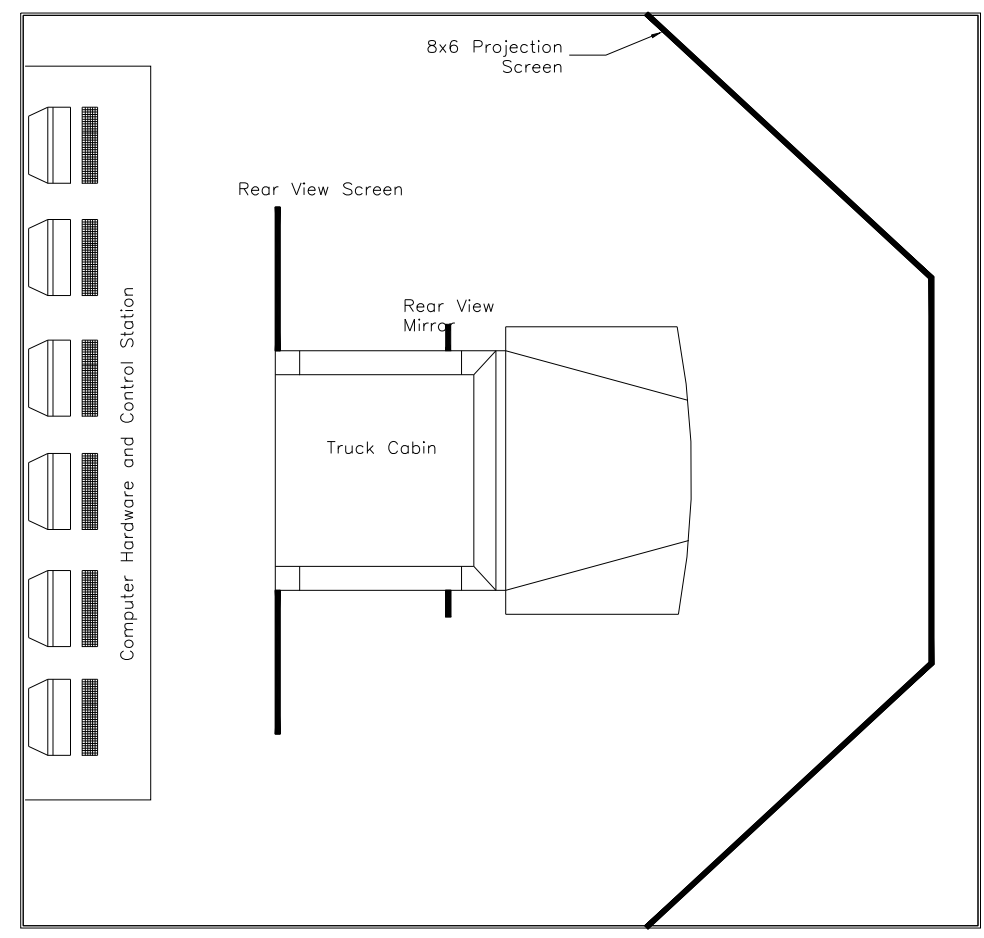

Figure 3.4: General layout of the driving simulator display system (continued)

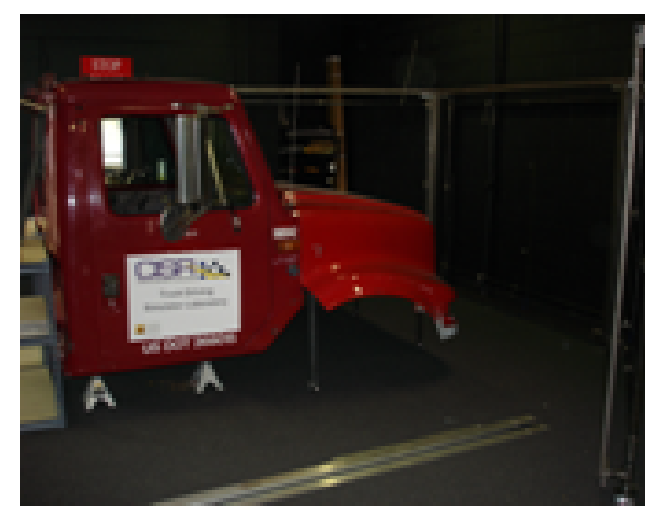

Figure 3.5: Driving simulator truck cabin

\section{Screens}

While curved screens theoretically produce better optic flow rendering, additional precautions are necessary to avoid image deformation and achieve correct alignments at image junctures. On the other hand, borders between screens potentially visible to the drivers are inevitable when using flat screens. An alternative is to place these borders behind the vehicle cabin pillars from the driver's perspective. By not seeing the border of each screen, the driver will have the impression of a single continuous image projected in front of him. 
Consequently, while curved screens represent the best solution for expensive highfidelity driving simulators, flat screens remain a good choice for low to medium fidelity simulators. For the fidelity required for these experiments, the research team believe that a simple screen design using white mat finish non-reflective display materials can achieve acceptable levels of performance.

The following basic rules must be considered to determine the screens positions (Figure 3.6):

- To avoid optical distortions, the driver should sit at equal distances from the center of each screen.

- The distance between the screens and the driver is crucial. Placing the driver too close to the screens results in large angles between the screens that compromise the driver's impression of seeing a single continuous image. On the other hand, placing the driver too far away from the screens limits the width of the scene observed by the driver. Driver's positioning is commonly considered adequate when the driver is placed 9 to 10 feet from the screens. The screens' orientation angles are obtained as a consequence of this requirement.

For the CISR new truck simulator, the research team adopted direct projection for the three front screens and back projection for the two rear screens.

More details on the design and fabrication of the screens are provided in Appendix B.
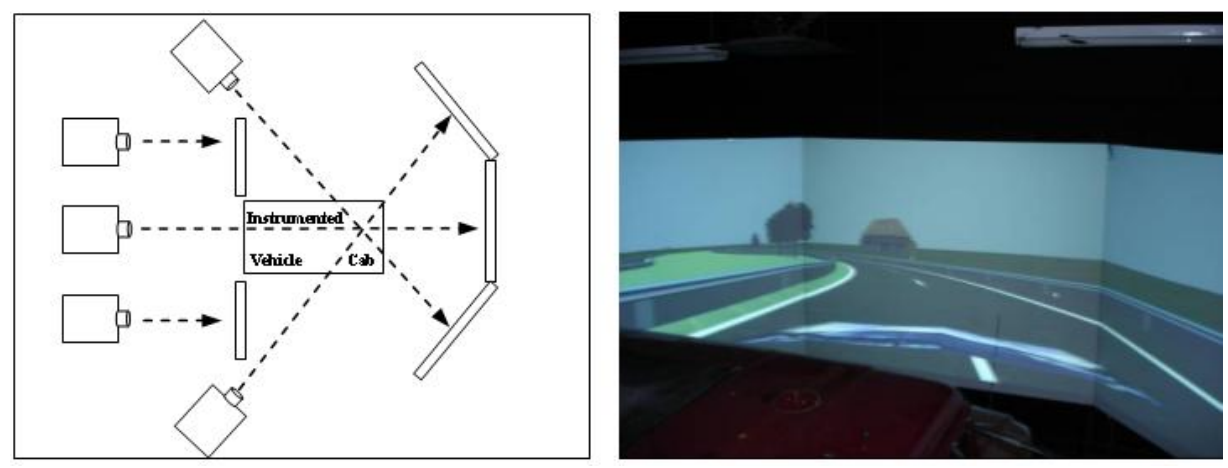

Figure 3.6: Front and rear projection in the truck driving simulator

\section{Computer Hardware}

Creating complex three-dimensional driving scenes involves highly demanding graphic applications that require significant computing power. The performance levels of both the processor and graphic cards are crucial for real-time generation of detailed virtual environments.

Seven networked PCs run this truck driving simulator. Five PCs (Intel Pentium 4 processors at $3.0 \mathrm{GHz}$ with $1024 \mathrm{MB}$ SDRAM) equipped with large memory video cards (128 MB ATI Radeon 9800 Pro) perform the highly demanding graphic computation of the projected three-dimensional driving scenes. One PC is interfaced with the truck cabin 
to compute vehicle dynamics, traffic and sound effects. The last computer manages and records signals from the eye tracker and video monitoring systems.

\section{Steering Feedback System}

Computer controlled DC motors provide one of the best options for steering feel system because they offer high output torques and good response time at reasonable costs. Faros Inc. of France supplied the steering system hardware. A DC motor produces the feedback torque. The CEGI controller card designed by INRETS hosting the steering feedback algorithm sends steering commands to the steering power module. This PulseWidth-Modulation driven unit then generates high current signals to control the torque motor, as shown in Figure 3.7. Delocalized computing of the steering response ensures good update rates of the vehicle response during simulation.
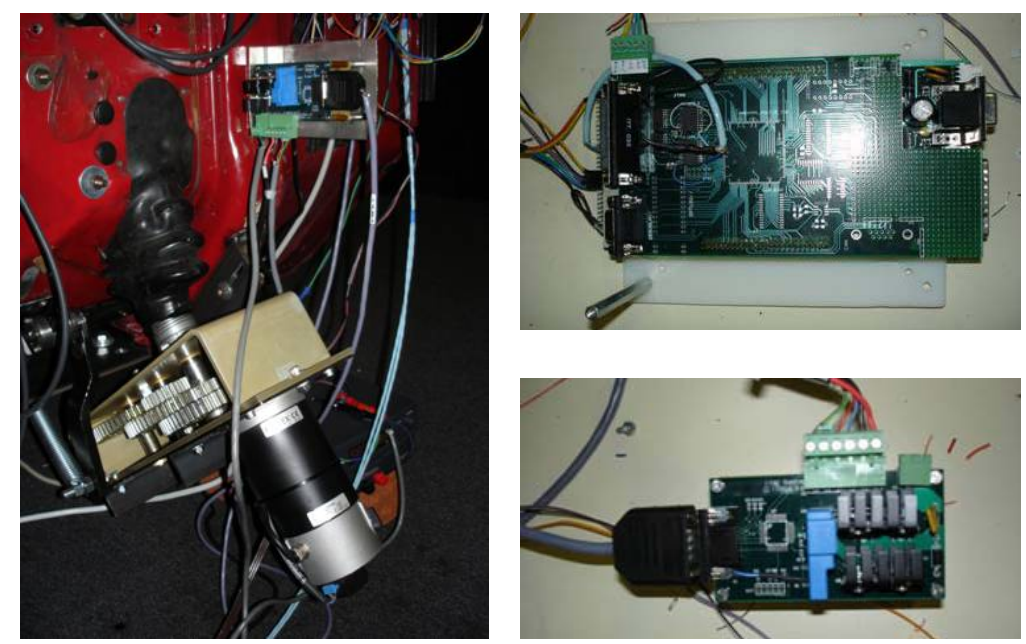

Figure 3.7: Faros steering feedback actuator and gears, INRETS CEGI control unit, and PWM power module

The torque actuator is connected to the steering shaft through a set of spur gears with a 7:1 gear ratio. The large gear ratio allows the amplification of torque ratio. To prevent the steering wheel from turning endlessly, a mechanical stopping mechanism is provided. A steel pin mounted on a gear prevents the steering wheel from turning more than 3.5 revolutions in each direction. Steering alignment errors can produce undesirable steering torque and cause premature wear-out of the steering elements. To compensate for small mounting errors, a flexible coupling is used to connect the steering shaft to the DC motor shaft. More details on the mounting of the steering torque system are described in Appendix B.

In the new truck simulator, the parameters of the steering torque response system were adjusted using subjective evaluations to achieve a realistic steering feel. More details are provided below in the section Validation of the Simulator. 


\section{Gearbox System}

The resistance of the gearbox system to driver's control inputs should be representative of a real transmission. Faros Inc. supplied the manual stick shift Gear Box system, shown in Figure 3.8. The new system is an adaptation of the INRETS gearbox system developed for a car simulator. The system is capable of simulating a 10-speed gear ratio manual stick shift transmission. A set of levers and springs create the feeling of gear shifting. Gear position is determined by a round steel pin locking into one of the six cavities machine in a steel plate. A flip switch located in the lever hand knob determines higher gear ratios. A set of three potentiometers is used for sensing the current gear position. Two potentiometers determine the longitudinal and lateral position of the round steel pin while a third potentiometer, located in the flip switch in the lever, determines the high speed gear position. The shift lever was modified and a mounting frame was fabricated to assemble the gearbox inside the vehicle cabin (see Appendix B).
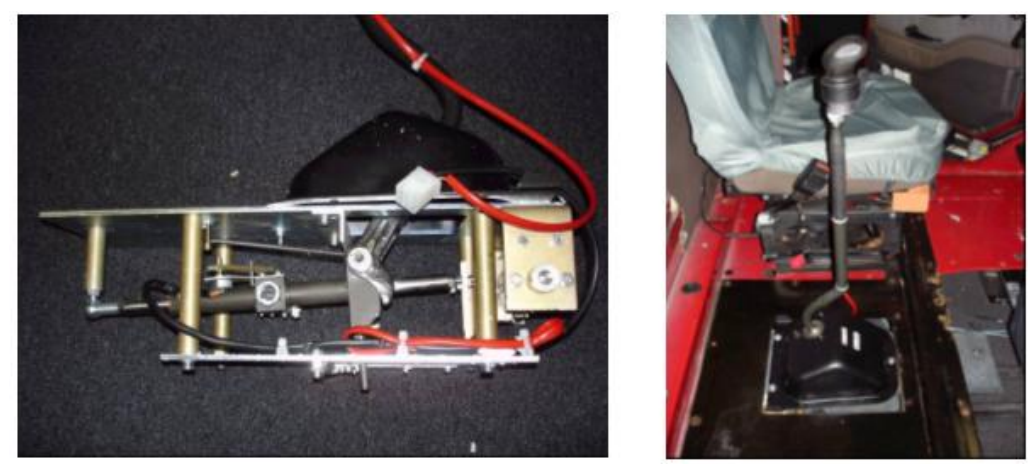

Figure 3.8: Faros Gear Box system and final assembly in simulator cabin

\section{Control Pedals}

To measure the driver's input to the cabin pedals during simulation, optical encoders were mounted on the throttle, brake, and clutch pedals, as shown in Figure 3.9. Incremental encoders with push-pull output were acquired. A resolution of 500 pulses per revolution was selected to ensure precise measurement of the pedal movements.

New pedal mechanisms had to be designed and fabricated to reproduce the pedal feel originally created by hydraulic systems. Passive spring and lever mechanisms were used to generate resistance in the pedals, which is described in detail in Appendix B. The installation of the optical encoders was designed to be transparent to the subjects when driving. Also, the position of the pedals is identical to the original cabin configuration.

\section{Cabin Adaptation and Instrument Interfaces}

Vehicle cabin instruments, controls and switches have to be integrated in the driving simulator and interfaced with the simulation software to reproduce the normal operation of a real vehicle and measure drivers' inputs during an experiment. 

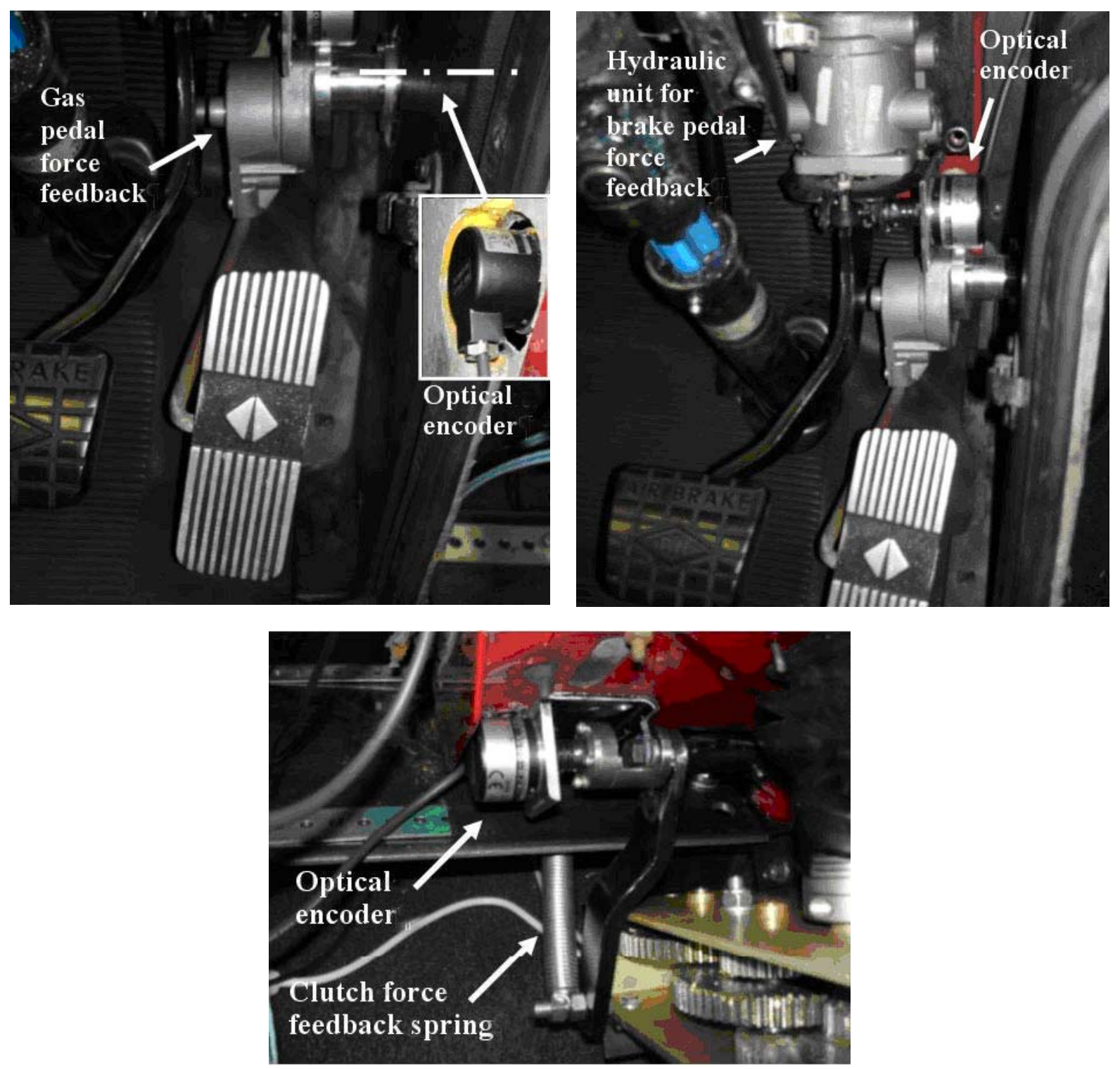

Figure 3.9: Optical encoders mounted on the truck cabin pedals

Electronic circuits and connectors were fabricated for appropriate interfacing of cabin sensors (i.e., optical encoders and potentiometers) and for operation of cabin instruments (i.e., cabin displays and dashboard gauges), as shown in Figure 3.10. Special hardware was also designed to host all communication and control cards.

Details on electronic adaptations and interface layers are provided in Appendix B. 

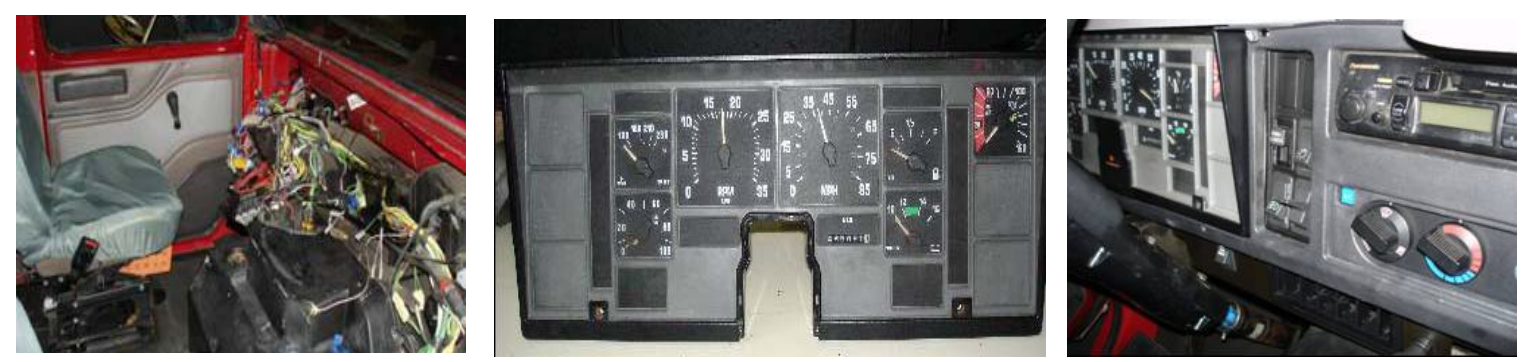

Figure 3.10: Cabin instruments wiring, dashboard gauges and instrument panel

\section{Sound System}

The INRETS sound generation algorithm is capable of generating both vehicle sounds (e.g., engine sounds during vehicle start and acceleration/deceleration) and sounds from surrounding traffic. The INRETS original engine sound samples were edited and modified to better resemble the sound rendering of big diesel truck engines.

A multi-channel sound system composed of five speakers and a subwoofer was installed in the new truck simulator. Each element was strategically placed in the cabin to optimize the spatial sound rendering, which is described in more detail in Appendix B.

\section{Driving Scenario}

Developing the right type of driving environment is crucial to the successful simulation of any driving task. Each element of the driving scenario has to be defined by the researcher according to the experimental protocol. These elements include definition of the roadway, surrounding environment and other static objects, vehicular traffic, all events that will occur during the experiment, and the type of data that needs to be recorded during the experiment. An improper or poorly defined scenario will only produce incorrect and useless data.

Because driving simulation has many limitations in terms of computing and graphic requirements, driving scenarios should be defined based on the unique sequence of tasks and events required to simulate the driving situation being studied.

When studying driver drowsiness, the driving scenario should mainly consist of long and monotonous driving sections that are likely to induce driver boredom and fatigue. Based on statistical data on the characteristics of accidents involving drivers' drowsiness, the following scenario was developed for the truck simulator:

- A rural interstate highway with speed limits of 55 to $65 \mathrm{mph}$ (70\% of crashes occur on this type of highways and within this range of speeds)

- Early morning and nighttime driving (more than 40\% crashes occur between $1 \mathrm{am}$ and $7 \mathrm{am})$

- Very little traffic and other activity in the surrounding areas representing a monotonous and calm atmosphere 
This scenario includes realistic sounds, visual effects, and other pre-defined events regarding road traffic and traffic controls.

CISR decided to develop a driving scenario based on a real interstate rural highway and acquired hard copies of plans and profile drawings from the Kansas Department of Transportation. These drawings cover a 52-mile section of Interstate 70. Alignment, traffic, and other environmental conditions on this highway are very consistent with that required for studies of fatigued/drowsy driving.

In collaboration with INRETS, CISR extracted geometric design information from the drawings (i.e., horizontal and vertical curvatures, cross-section data, marking information, etc.) and created a three-dimensional roadway environment using the OpenGL Performer graphics library from Silicon Graphics. Vehicles, traffic signs, and roadside and background objects were then added to the model, as shown in Figure 3.11. An exhaustive sequence of driving actions and traffic events was defined to complete the driving scenario. More details on the creation of the driving geometric database and three-dimensional objects of the driving scenario are provided in Appendix B.

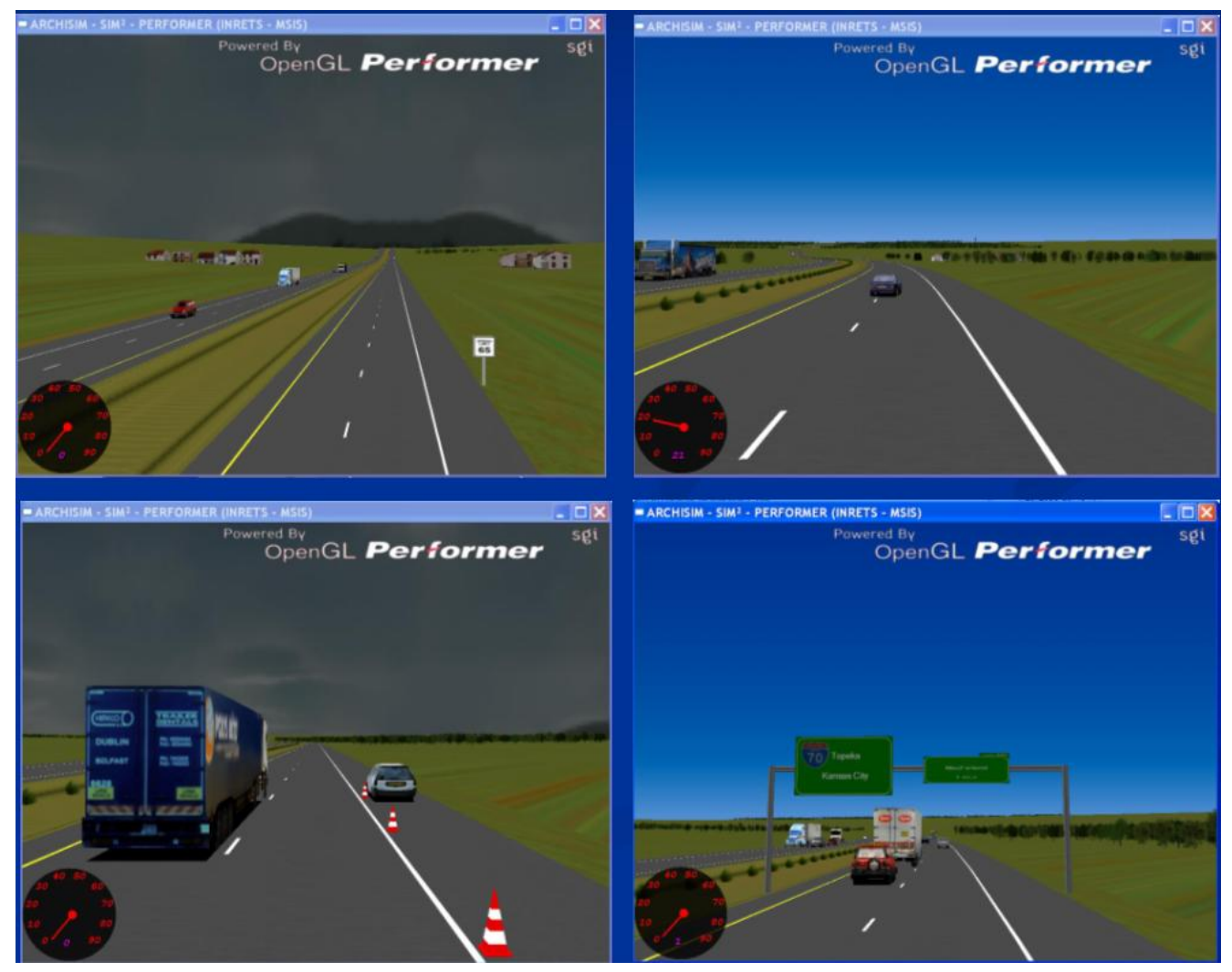

Figure 3.11: 3D scenes from I-70 driving scenario with environment and traffic

\section{Equipment for Data Acquisition and Driver Drowsiness Measures}

Additional hardware was required in the truck driving simulator to measure and record driver's behavior and level of alertness/sleepiness during the drowsiness studies. 
Video images provide valuable information for studying driver behavior. The truck driving simulator is equipped with a digital video monitoring and recording system. Infrared cameras are placed in the truck cabin, as shown in Figure 3.12. These cameras capture images of the driver's face, body, hands, and feet, and displayed the driving scene as viewed from the inside the cabin, shown in Figure 3.13. A digital multiplexer is used to manage all video streams simultaneously. A $500 \mathrm{~GB}$ storage unit and a DVD-burner are used to store the recorded data.

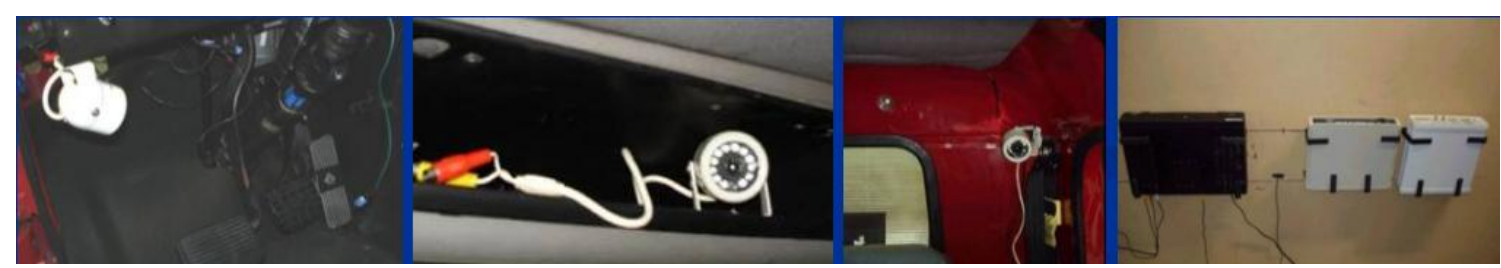

Figure 3.12: Infrared cameras for foot and pedal movements, driver's face and hands movements, and digital multiplexer

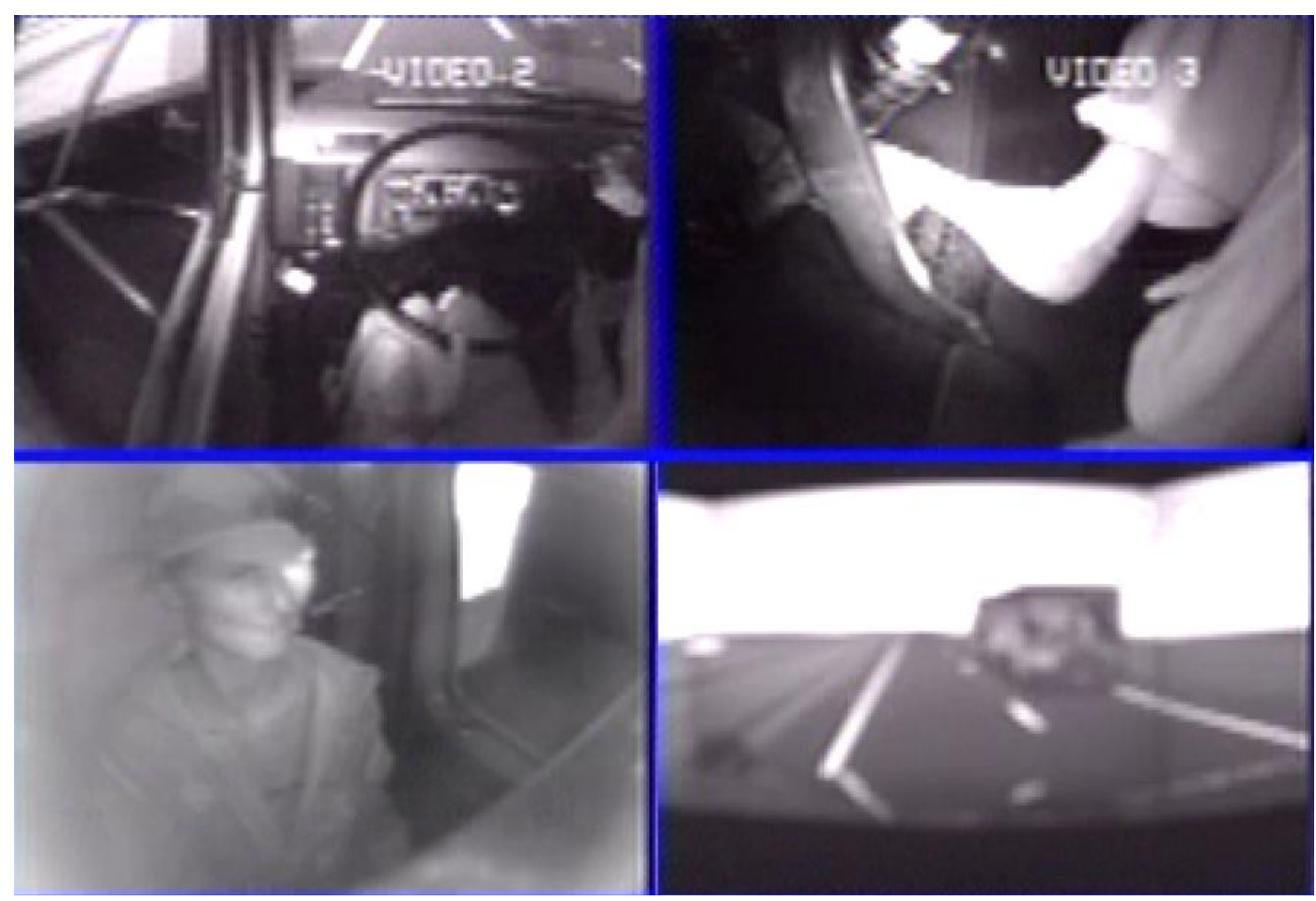

Figure 3.13: Infrared images of driver's body, hands, feet and driving scene

The simulator lab is also equipped with an eye tracking and measuring system. The system measures and records the line of gaze and the pupil diameter of the driver during experiment. Figure 3.14 shows a schematic of the Eye-Head Tracking system.

The beam from a near infrared source illuminates the eye and the optical system focuses an image of the eye onto a solid-state video sensor (eye camera). Both the illumination beam and the eye image are reflected from a visor. The control unit processes the eye 
camera signal to extract the elements of interest (pupil and reflection of the light source on the cornea) and computes both pupil diameter and line of gaze. Pupil and corneal reflection outlines and center cross hairs are displayed on the pupil monitor over the video image of the eye. Eye line of gaze with respect to the helmet is displayed as a cursor or set of cross hairs superimposed on the scene camera video image. Calibration commands and most other interaction with the operator take place through the Eye Tracker Interface PC terminal.

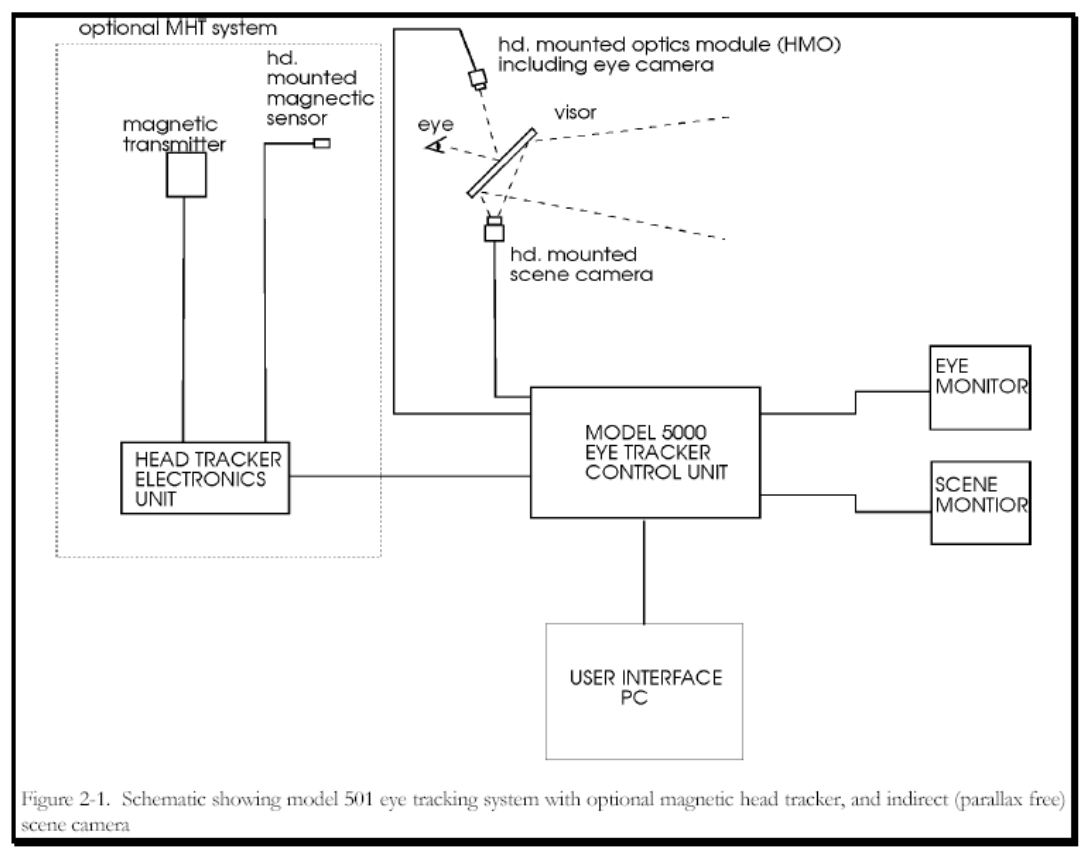

Figure 3.14: Eye tracking system schematic

\section{Validation of the Simulator}

The last phase of the truck driving simulator setup consisted of adjusting the system parameters to achieve realistic truck driving rendering and validating the new simulator for driver drowsiness studies based on subjective evaluations.

\section{Adjustment of the Vehicle Dynamic Model and Simulated Traffic}

To get a realistic truck driving rendering in various vehicle controls, especially the steering, the vehicle parameters of INRETS passenger car model had to be modified. The vehicle inertial parameters, the suspensions and tires constants, and the steering and gearshifting characteristics of an International 8100 truck were entered in an INRETS model. Because the experiments to be conducted in the simulator do not involve driving situations with severe dynamic vehicle responses, this approach, when supplemented by a rigorous verification of the resulting truck behavior, was adequate for this study.

\section{Testing the Simulator}

After completing the installation of hardware and software, the simulator components had to be tested for accuracy, reliability, and authentication of data acquisition. Simulation under fully loaded conditions were performed for prolong periods of time to test the 
system for reliable performance. Potential hardware weak points were identified and replaced by alternative solutions. For example, the gearbox wiring design was modified to prevent future cable breakage.

Many problems can also arise during operation of any distributed and/or networked system due to network congestion, memory usage, or other conflicts, which can cause the entire simulation to crash. The system was tested for all these problems, and modifications and reconfigurations were made for continuous system operation. For example, anomalies in the computation of traffic vehicle interaction were corrected, and problems of computational overload due to excessive graphic demand were solved.

\section{Subjective Evaluation}

Evaluation of the simulator was performed based on subjective evaluation by experienced personal. Because driving feeling differs between heavy commercial vehicles depending on their type, brand and model, size, load, etc., the new simulator had to produce a driving feeling representative of an average heavy truck. CISR asked three professional truck drivers to drive the simulator, and based on their advice, the following parameters were adjusted for a more universal truck driving feeling: steering ratio, maximum steering angle, steering torque, total vehicle load, maximum braking torque, and gear ratio.

CISR then asked other CMV drivers to subjectively evaluate the CISR truck simulator after adjustments to the above parameters were performed. Each of the drivers completed a practice session and was asked to complete a questionnaire regarding performance of various controls and functions of the simulator. Drivers were asked to evaluate the overall realism, responsiveness, and feel of the simulator steering, gearbox, and pedals systems. Additional measures included:

- Sound Intensity

- Sound Direction

- Sound Overall Realism

- Display Graphics

- Display Size/View

- Display Overall Realism

- Instruments Overall Realism

- Scenario Vehicles

- Scenario Roadway
- Scenario Surroundings

- Scenario Traffic

- Scenario Overall Realism

- Cabin Driver Seat

- Cabin Overall Interior

- Cabin Overall Realism

- Side Mirrors Size

- Side Mirrors View

- Side Mirrors Overall Realism

Overall drivers' evaluation averaged 1.8 on a scale of 1 to 5 ( 1 being very close to real truck and 5 being very unrealistic). The overall average response for the steering performance was 1.7. 
The last task in the truck simulator development was the validation of the simulator for the driver's drowsiness studies. CISR invited a panel of experts from the driving simulation and human factors research fields to assess the new laboratory set up for drowsiness studies. The new simulator received very positive feedback from the experts. Minor recommendations were taken into account and corresponding adjustments were implemented in the driving simulator. 


\section{EXPERIMENT AND DATA COLLECTION}

One of the primary objectives of this project was to conduct driving simulator experiments in order to collect data on driver behavior. This data is then used to develop detection model for driver fatigue/drowsiness specific to the truck driving environment. This chapter describes the experiments conducted, data collected, and statistical analysis of the data.

\section{OVERVIEW OF THE DROWSY DRIVING EXPERIMENT}

The experiment was conducted at the CISR Truck Simulator Laboratory located in the George Washington University campus in Ashburn, VA. During this experiment, truck drivers drove a truck simulator under various levels of fatigue and sleep deprived conditions. Data was recorded for parameters related to driver eye activity and vehicle driving performance. Details of the study population, equipment used in the experiment, driving scenario, and experimental protocol are provided in the following sections.

\section{Study Population}

The subject drivers were recruited through advertisements in local newspapers, The Trucker magazine, and by soliciting companies involved in freight and trucking business. More then 40 drivers responded; 20 of them consented and completed the practice session. However, only 14, including two women, were able to complete the actual experiment. All of the subjects were truck drivers with a valid Commercial Drivers License (CDL). The subjects represented a variety of truck driving professionals such as owner operators, company drivers, and freelance part-timers. Driver age ranged from 23 to 60 years with an average of 42 years, as shown in Figure 4.1. Subjects were screened for heavy intake of caffeine, drugs, sleeping disorders, and susceptibility to simulator sickness before being allowed to participate in study. Subjects were generally nonsmokers and low caffeine users, and they did not use stimulants including caffeine throughout the study.

Drivers were selected based on a brief telephone interview after which they visited the laboratory. Each participant was asked to fill out two questionnaires regarding their general health and their susceptibility to simulator sickness. Those who did not fulfill the criteria were screened out. Limited demographic information (e.g., age, gender, driving experience, health, etc.), was also collected. The subjects were required to adhere to a regular sleep routine with at least 8 hours of sleep before the experiment. Prior to any driving session, each subject was required to read and sign an informed consent form and an audio video release form. Brief personal information was collected. At the end of the experiment, subjects were paid $\$ 350$ for their time and efforts. 


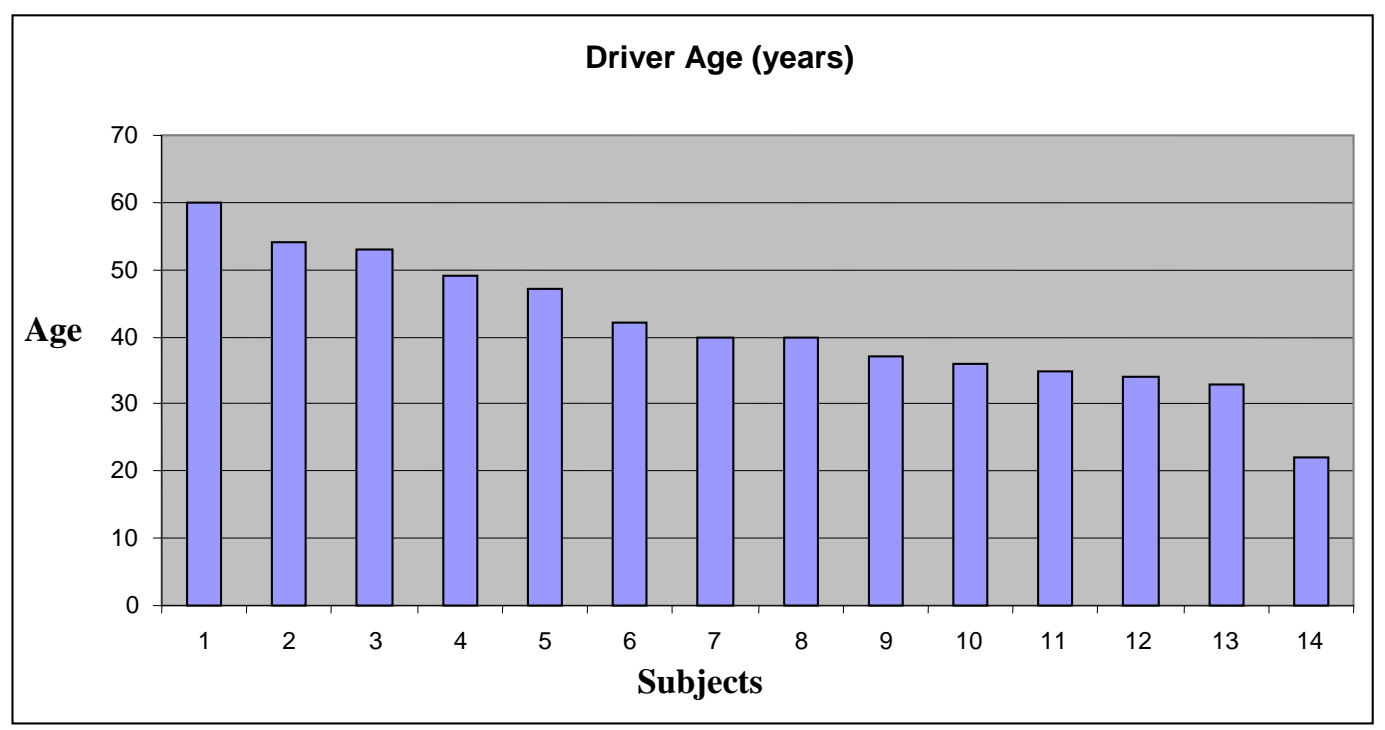

Figure 4.1: Drivers' age

\section{Equipment}

Experiments were conducted in the CISR Truck Driving Simulator Laboratory (TDSL) at The George Washington University campus in Ashburn, VA. TDSL is a high fidelity, fully interactive, fixed base driving simulator. It is organized around a full-size truck $\mathrm{cab}$, with a computer generated image projected onto a 135 degree front screen system. A partnership was established between CISR and the Modeling, Simulation and Driving Simulators (MSIS) research unit of the French National Institute for Transport and Safety Research (INRETS) to develop this simulator. This simulator was built in-house specifically for this study. Details of the simulator are provided in the previous section as part of TASK 3. A head-mounted eye tracking and measuring system from Applied Science Laboratory (ASL) was used to monitor driver eye activity.

\section{Driving Scenario}

The driving scenario consisted of a continuous 52-mile interstate highway. This scenario represents a portion of interstate 70 in the state of Kansas and extends from Junction City to Topeka, KS. Alignment, traffic, and other environmental conditions on this highway are very consistent with that required for fatigued/drowsy driving. The selection of this section of the highway was not based on accident data and no specific accident data was available. Rather, it was the availability of design data and the rural driving environment that lead to the selection of this section of highway. Details about the development of this scenario are provided in the previous section and in Appendix B.

\section{Experimental Protocol}

The protocol consisted of testing the driving performance of truck drivers under fatigued and non-fatigued conditions in a driving simulator. There were a total of three simulator driving sessions: a practice session, a morning session, and a night session. 


\section{Practice Session}

Drivers who responded and showed interest were contacted and briefly interviewed on the phone for preliminary screening. Those who qualified were asked to visit the GWU campus in Ashburn, VA for the informed consent process and a practice driving session. As required by the Institutional Review Board (IRB), drivers were asked to read an inform consent form and, if they agreed, sign the form. They also filled out a questionnaire regarding personal information and simulator sickness. Drivers then took a tour of the simulator laboratory and were briefed about the experiment and operation of the simulator; all the functions and driving controls were explained to them. Each subject was given detailed written instructions of what was required of her/him in the experiment. They were told to follow all the rules of the road and maintain the instructed speed limit. They then drove the simulator for one full length of the scenario and they were scheduled for the actual experimental session on the day of their availability. Each driver was required to complete two experimental driving sessions, a morning session and a night session. Table 4.1 shows the amount of sleep deprivation and time schedule for the two experimental sessions.

Table 4.1: Amount of sleep deprivation

\begin{tabular}{|c|c|c|}
\hline & Morning Session & Night Session \\
\hline $\begin{array}{c}\text { Amount of continuous } \\
\text { wakefulness }\end{array}$ & $1-2$ hours & $18-19$ hours \\
\hline Time schedule & $8: 30 \mathrm{am}-9: 30 \mathrm{am}$ & $1: 30 \mathrm{am}-5: 00 \mathrm{am}$ \\
\hline
\end{tabular}

\section{Morning Session}

Subjects were instructed to have at least 8 hours of sleep the night before their scheduled testing date. They reported to the simulation laboratory or were picked up by a researcher in the morning, 30 minutes before the start of the experiment. In the morning experimental session, subjects drove the simulator for one full length of the scenario (i.e., 52 miles). During this session, subjects were fresh and experienced no fatigue due to sleep deprivation. After completing the morning experimental driving session, they were allowed to carry on with their daily life activity. Subjects were asked to have a limited amount of caffeine intake and no sleep during that day. They then reported back to the laboratory the same night for the night session.

\section{Night Session}

For the night session, subjects were picked up by the researcher from their home or hotel, and they arrived at the laboratory 2 hours before the start of the experiment. Subjects stayed at the laboratory till the start of the experiment and were monitored for falling asleep. A member of the research team remained with the subjects during this time to keep them awake and provide any assistance. The night experimental session started at 1:30 am and continued until 5:00 am or until the driver was too drowsy/fatigued to 
continue driving. Most of the drivers stopped before 5:00 am. The same 52 mile driving scenario was continuously repeated. In this session, subjects were sleep deprived and were susceptible to fall asleep while driving the simulator. After completing the two experimental sessions, subjects were debriefed and asked to fill out a questionnaire regarding their general condition and simulator sickness. Subjects were driven home and were not allowed to drive since they were sleep deprived. To insure the safety and health of the participants, a paramedic was present at the CISR laboratory throughout the experimental session.

\section{DATA COLLECTION}

Three types of data were collected during the experiments: data related to vehicle parameters, eye closure data, and video images. An overview of this data is provided in this section.

\section{Vehicle Parameter Data}

The vehicle parameter data included speed, acceleration, vehicle lane position, steering angle, braking, and heading angle. This data was recorded at a frequency of $20 \mathrm{~Hz}$ from sensors through simulation software and stored as an ASCII file. There were no problems associated with recording except for one subject whose vehicle parameter data was not properly recorded. This subject's data was not used for data analysis and in the model development.

\section{Eye Closure Data}

Eye closure data was recorded at $60 \mathrm{~Hz}$ through equipment by ASL. This system records pupil diameter by capturing reflection from pupil (bright pupil). Subjects have to wear a headband carrying a camera and a source of infrared light. Before the start of an experiment, a researcher adjusts the reflecting glass and camera to get a clear picture of the eye in the field of view of the camera. The system then tracks the brightest image in the frame, which is reflection from the pupil. The system records a value corresponding to pupil diameter but will record a zero value every time it looses track of pupil due to eyelid closure or any other reason. There were many problems associated with eye data recording due to driver behavior and system shortcomings. They include:

- Not performing with two types of glasses. (Glasses with shiny frames, since the reflection from the frames caused it to track the shiny spots rather than the pupil, and glasses with certain types of coated lenses that also caused reflection from the lens rather than the pupil.)

- The movement of drivers' pupils to extreme positions caused the pupil to partially disappear, resulting in the system loosing track of the pupil. This occurred when some drivers looked left or right to check their side mirrors. 
- Misaligning the reflecting mirror, which caused the system to lose track of the eye. This was due to some drivers, who scratched their face or rubbed their eyes, particularly during night sessions.

Every time the system loses track of the pupil, it records a zero value as it does when the eye is closed. Eye closure data for some subjects was not usable while portions of eye data for others had to be discarded. Given the very high volume of recorded data and the availability of video images of the driver's face, discarding erroneous data due to eye tracker system malfunctioning was not an issue for developing the drowsiness detection model and consequently did not affect the final results of this study.

\section{Video Images}

As already detailed in Chapter 3 of this report, a digital video recording system was used to monitor driver's behavior inside the vehicle cabin during experiment. Infrared cameras recorded the driver's face, steering wheel and instrument panel including the driver hands, driver's foot position, and roadway scene from inside the cabin. Data was stored on a separate high capacity hard drive and was later transferred to DVDs and CDs for backup purposes.

\section{DATA ANALYSIS}

Before a drowsy driver detection system can be developed, the data from the experiments is analyzed to identify the potential variables that are correlated with drowsiness. This section first reviews the characteristics of drivers' drowsiness, the manner in which it could be identified, and how other factors such as fatigue or monotony can also play a role in driving performance degradation. Data collected during experiments on drivers and their driving outcomes are then individually analyzed. The objective here is to identify variables correlated with driver drowsiness. Such variables can then be used either as input for a drowsy driver detection system or to detect drowsiness for the evaluation of the performance of such a system.

\section{Identification of Driver Drowsiness}

In order to assess the effectiveness of a drowsiness detection system, it is essential that driver fatigue can be identified. The sleep-wake cycle is a primary factor of driver drowsiness, and it was used to design the parameters of the night session in order to increase the likelihood of drowsiness. Nonetheless, it is essential that the assessment of a drowsiness detection system differentiate between fatigue and monotony, both of which can cause driver performance degradation. This experiment differentiated between driver fatigue and monotony through real-time observations during the experimental sessions, off-line video recording, and eye closure data.

\section{The Sleep-Wake Cycle}

The sleep-wake cycle informed the design of the experimental protocols, which were designed to increase the likelihood of driver fatigue during the night driving sessions. 
The sleep-wake cycle is regulated by homeostatic and circadian factors (NCSDR/NHTSA Expert Panel on Driver Fatigue and Sleepiness, 1998). Homeostatic factors are related to the neurobiological need to sleep while the circadian pace maker is an internal body clock that governs sleeping patterns. The tendency to sleep during sleep cycle is inevitable and intrinsic. Falling into sleep is neurobiological responses of the human brain to sleep deprivation.

According to a survey of truck drivers conducted after the practice driving tests, most of the drivers indicated that their "tiredness peak time" varies between 2 am and $4 \mathrm{am}$. The survey outcome initiated a test schedule modification in starting test time from 12:30 am to 1:30 am. In another survey after night session tests, some drivers also stated that their drowsiness level and dozing off experiences during driving diminished after they passed the "tiredness peak time". In addition, they indicated that their dozing off incidents were cyclic and sporadic experiences.

\section{Differentiating Between Fatigue and Monotony}

As described in the Driving Scenario section in Chapter 3, the simulation scenario was intentionally monotonous to increase the likelihood of driver fatigue. Another consequence of selecting a monotonous driving scenario is that it can decrease drivers' attention and affect their awareness of the driving situation. Driving performance can, therefore, be degraded even for alert drivers. In addition, assuming that drivers' fatigue is related to the time spent at the driving task, fatigue was also considered as one of the major sources of driving performance degradation during the long driving periods of the night sessions.

Thus, there are two possible candidates, drowsiness/fatigue and monotony, which could be responsible for any driving performance degradation. According to the drivers' responses to the questionnaires handed over after completion of each night session, $75 \%$ of tested drivers had moderate to severe fatigue symptoms, while $36 \%$ of the subjects had moderate to severe boredom symptoms after the test. The results suggested that monotonous driving had less influence on driving performance than the fatigue and drowsiness factors. During data analysis, to correlate the various recorded variables to drowsiness, the causes of the driving performance degradation were carefully examined through different indicators, such as the video recordings, to make sure that drowsiness was the dominant effect.

\section{Assessing Driver Drowsiness}

The drowsiness of the tested drivers is identified and validated in three ways:

- Real-time observation during testing: The driver behavior, performance, and eye closure were observed during testing through live video monitors. All behavioral signs indicating a state of drowsiness were observed and recorded. The records were especially useful for identifying driver drowsiness when other methods like eye monitoring experienced measuring problems (see limitations of the eye tracker system in the Eye Closure Data section in this chapter). 
- Off-line video recording: Drowsiness was also observed in video recordings from each driver's face. Driver drowsiness was identified based on subjective judgment from drowsy facial attributes and eye closure observation. This method was used to confirm PERCLOS data.

- Eye closure measures (PERCLOS): PERCLOS quantifies the percentage of time the eye is more than $80 \%$ closed. This measure indicates the intervals of time when the eyes were closed. To check and confirm the eye closure data and avoid corrupted data, the data was validated by other methods, especially by video data.

\section{Eye Closure Data (PERCLOS)}

PERCLOS, the percentage of time the eye is more than $80 \%$ closed, is one of the most widely accepted measures in scientific literature for drowsiness detection. It has been validated using both EEG data and subjective evaluation. One of the limitations of the PERCLOS model is that its prediction is good only when using large time intervals. Moreover, PERCLOS does not take into account the variability in human behavior as blinking activity can significantly differ between individuals.

In addition, as discussed in the section on Eye Closure Data in this chapter, the technology used to monitor eye motion can present some limitations and generate invalid results. In this experiment, some portions of the eye data had to be discarded for some drivers because the eye tracker system lost track of the eye pupil during the experiment. Figure 4.2 displays PERCLOS 30-minute average for each subject. According to the figure:

- Each subject who crashed and/or displayed drowsy behavior based on the video data, shows significant difference between the morning and night eye closure data plots.

- During all morning sessions, the value of PERCLOS was below 12\%. Eye closure varies among drivers. Some people tend to have longer eye closure during alert driving than during drowsy driving. Therefore, the threshold for differentiating between alert and drowsy states for each subject can be based on the average of each subject's eye closure during the morning session. Table 4.2 shows the PERCLOS average for the subjects with no data recording problems.

- The morning plots did not vary significantly with respect to time.

- PERCLOS 30-minute average curves for night sessions had local maxima and minima indicating that eye closure did not steadily increase throughout the night session. 
Table 4.2: Mean PERCLOS for morning sessions

\begin{tabular}{|c|c|}
\hline Subject No & Mean PERCLOS \\
\hline 2 & 0.06 \\
\hline 8 & 0.03 \\
\hline 9 & 0.12 \\
\hline 10 & 0.07 \\
\hline 14 & 0.06 \\
\hline 16 & 0.04 \\
\hline 17 & 0.01 \\
\hline 19 & 0.08 \\
\hline 20 & 0.1 \\
\hline Average & $\mathbf{0 . 0 6}$ \\
\hline
\end{tabular}

\section{Correlation of Performance Variables with Drowsiness Development}

A statistical analysis of the data collected during the experiment helped to study the relationship between the different driver performance variables and the development of drowsiness. Variables that are highly correlated could be either appropriate inputs for a drowsiness detection system or enable the validation of the system by distinguishing between drowsy versus non-drowsy behavior. For example, steering activity at the onset of drowsiness would be an appropriate input for the system, if a correlation with drowsiness exists. On the other hand, the occurrence of an accident would not be an appropriate input, since the purpose of the system is to avoid accidents. If it is correlated with drowsiness, though, accidents could be an appropriate criterion to differentiate between drowsy and non-drowsy behavior. Table 4.3 shows a list of variables for which data was collected during the experiment. The analysis of these variables was completed in two phases. 


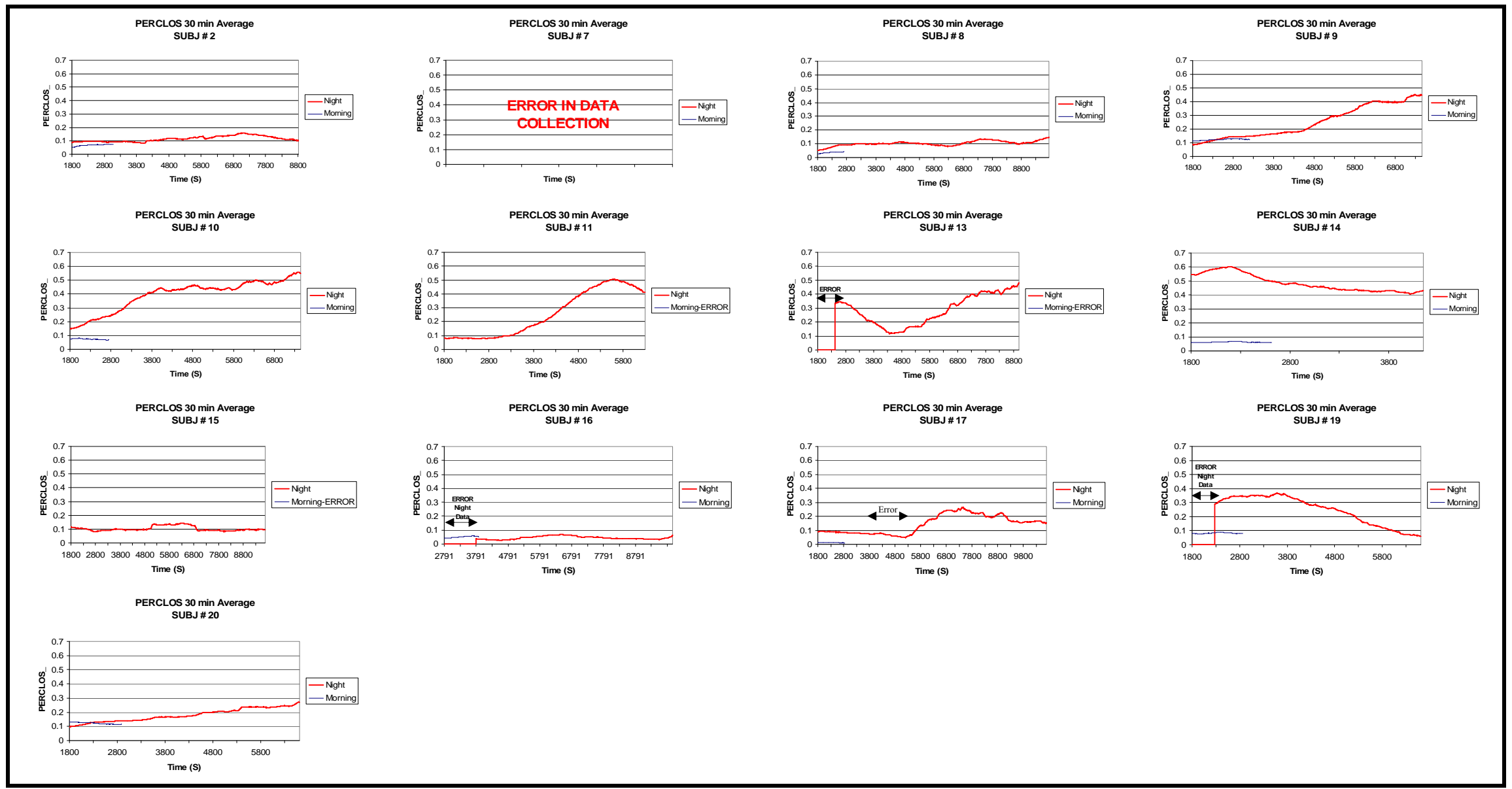

Figure 4.2: PERCLOS 30-minute average for morning and night sessions 
Table 4.3: List of recorded variables

\begin{tabular}{|c|c|}
\hline VARIABLE & DESCRIPTION \\
\hline SUBJ\# & Subject number from 1 to 20 \\
\hline AGE & Age of the subject in years \\
\hline GENDER & Subject gender (male/female) \\
\hline $\mathrm{ACC}$ & Vehicle acceleration \\
\hline ACC MEAN & Mean vehicle acceleration \\
\hline SPEED & Instantaneous speed \\
\hline SPEED MEAN & Mean speed \\
\hline SPEED STDEV & Speed standard deviation \\
\hline LATERALP & Lateral displacement from road centerline \\
\hline LATERALD & Lateral displacement from center of driving lane \\
\hline LATERALD_MEAN & $\begin{array}{l}\text { Mean absolute lateral displacement (from center of driving } \\
\text { lane) }\end{array}$ \\
\hline LATERALD_STDEV & $\begin{array}{l}\text { Absolute lateral displacement standard deviation (from center } \\
\text { of driving lane) }\end{array}$ \\
\hline HEAD & Absolute vehicle heading angle \\
\hline HEAD_MEAN & Mean vehicle heading angle \\
\hline HEAD_STDEV & Vehicle heading angle standard deviation \\
\hline GAS & Percentage of full throttle opening \\
\hline GAS_MEAN & Mean percentage of full throttle opening \\
\hline GAS STDEV & Percentage of full throttle opening standard deviation \\
\hline BRAKE TAP & Number of brake pedal taps/application \\
\hline T_CROSS & $\begin{array}{l}\text { Total driving time on or beyond the left/right shoulder or } \\
\text { center line }\end{array}$ \\
\hline T_CROSS_L & Total driving time on or beyond the left shoulder line \\
\hline T_CROSS_C & $\begin{array}{l}\text { Total driving time on or beyond the dashed line between travel } \\
\text { lanes without using the turning signal }\end{array}$ \\
\hline T_CROSS_R & Total driving time on or beyond the right shoulder line \\
\hline N_CROSS & Total number of left/right shoulder or center line crossings \\
\hline N_CROSS_L & Total number of left shoulder line crossings \\
\hline N_CROSS_C & $\begin{array}{l}\text { Total number of the dashed line between travel lanes crossings } \\
\text { without using the turning signal }\end{array}$ \\
\hline N_CROSS_R & Total number of the right shoulder line crossings \\
\hline TTC & Time to collide the leading vehicle \\
\hline CRASH_RD_L & Number of left run-off-road crashes \\
\hline CRASH_RD_R & Number of right run-off-road crashes \\
\hline CRASH_V & Number of crashes with another vehicle or barrier \\
\hline STEER & Steering wheel angle value \\
\hline STEER_E & Steering wheel angle instantaneous power \\
\hline STEER_P_MEAN & Mean steering wheel angle instantaneous power \\
\hline STEER_P_STDEV & Steering wheel angle instantaneous power standard deviation \\
\hline PERCLOS & Proportion of time that the eyes are closed in a second \\
\hline MDL & Lane mostly driven \\
\hline
\end{tabular}


In the first phase, the general trend of each variable was studied by analyzing total mean, total standard deviation, 30-minute average, and 30-minute standard deviation graphs. Thirty minute averaging was selected to smooth the data series by averaging every $30-$ minute data intervals into a data point immediately following the interval. The same process was also applied for the standard deviation graphs which depict the standard deviation for each 30-minute interval. This procedure makes it easier to spot trends and eliminates data volatilities and short time fluctuations. The 30-minute window was chosen because it creates smoother curves comparing to other smaller time lengths. Note that 30-minute average or standard deviation graphs, starts at 1,800 seconds (30 minutes) as no 30-minute base data is available before 30 minutes of simulation.

In the next phase of the analysis, if the general trend of a variable showed a distinguishable behavior change between morning and night sessions, the correlation of that variable to driver drowsiness was also examined. The variation of a particular variable was studied during selected drowsy periods to establish any correspondence between this variable and drowsiness.

This analysis identifies two variables that are particularly useful for the development of a drowsy driver detection system. Steering activity is highly correlated with drowsiness and would make an appropriate input for such a system. The occurrence of an accident is also highly correlated with drowsiness and is especially useful for the evaluation of the ability of a drowsy driver detection system to identify fatigued drivers.

\section{Age and Gender}

Two out of thirteen drivers who participated in this study were females. Therefore, no statistically significant conclusion can be made on the effect of gender on drivers' drowsiness. Since drivers' ages were not uniformly distributed over age categories, the potential effect of age on drowsiness likewise could not be analyzed.

\section{Speed}

Among 77\% of the subjects, mean speed (SPEED_MEAN) for the night session shows a slight increase comparing to the morning session while speed standard deviation (SPEED_STDEV) indicates no significant trend, as shown in Table 4.4. Instantaneous speed (SPEED) 30-minute average graphs for all subjects on Figure 4.3 display neither a significant nor a drowsiness-correlated change during night sessions.

Speed limit signs were posted along the entire road in the simulation scenario, governing drivers' traveling speed strategies. However, in Figure 4.3, a slight increase of mean speed was observed during night sessions as compared to morning sessions. This increase was not significant to a level that can clearly discriminate between the drowsy and awake states.

The testing sessions were terminated when drivers were too sleepy to continue driving. Therefore, the amount of data collected for each subjects was different. Figure 4.3 and other figures in the following sections show different time scales as the time driven in the simulator differed for each driver tested. 
Table 4.4: Speed mean and standard deviation for morning and night sessions

\begin{tabular}{|c|c|c|c|c|}
\hline & \multicolumn{2}{|c|}{ SPEED_MEAN (mph) } & \multicolumn{2}{c|}{ SPEED_STDEV (mph) } \\
\hline SUBJ\# & Morning & Night & Morning & Night \\
\hline 2 & 62.0 & 65.1 & 4.9 & 4.0 \\
\hline 7 & 55.0 & 59.5 & 7.6 & 7.2 \\
\hline 8 & 70.2 & 72.5 & 5.1 & 5.4 \\
\hline 9 & 59.5 & 59.3 & 5.6 & 3.8 \\
\hline 10 & 69.6 & 76.3 & 10.1 & 5.6 \\
\hline 11 & 67.1 & 72.5 & 6.3 & 7.8 \\
\hline 13 & 66.0 & 63.8 & 4.9 & 4.3 \\
\hline 14 & 72.9 & 73.8 & 9.8 & 13.2 \\
\hline 15 & 62.2 & 66.4 & 6.9 & 5.9 \\
\hline 16 & 66.2 & 76.1 & 7.2 & 8.5 \\
\hline 17 & 66.9 & 70.5 & 6.9 & 2.7 \\
\hline 19 & 66.7 & 69.3 & 8.3 & 8.1 \\
\hline 20 & 66.2 & 64.9 & 5.0 & 2.6 \\
\hline Average & $\mathbf{6 5 . 4}$ & $\mathbf{6 8 . 5}$ & $\mathbf{6 . 8}$ & $\mathbf{6 . 1}$ \\
\hline
\end{tabular}




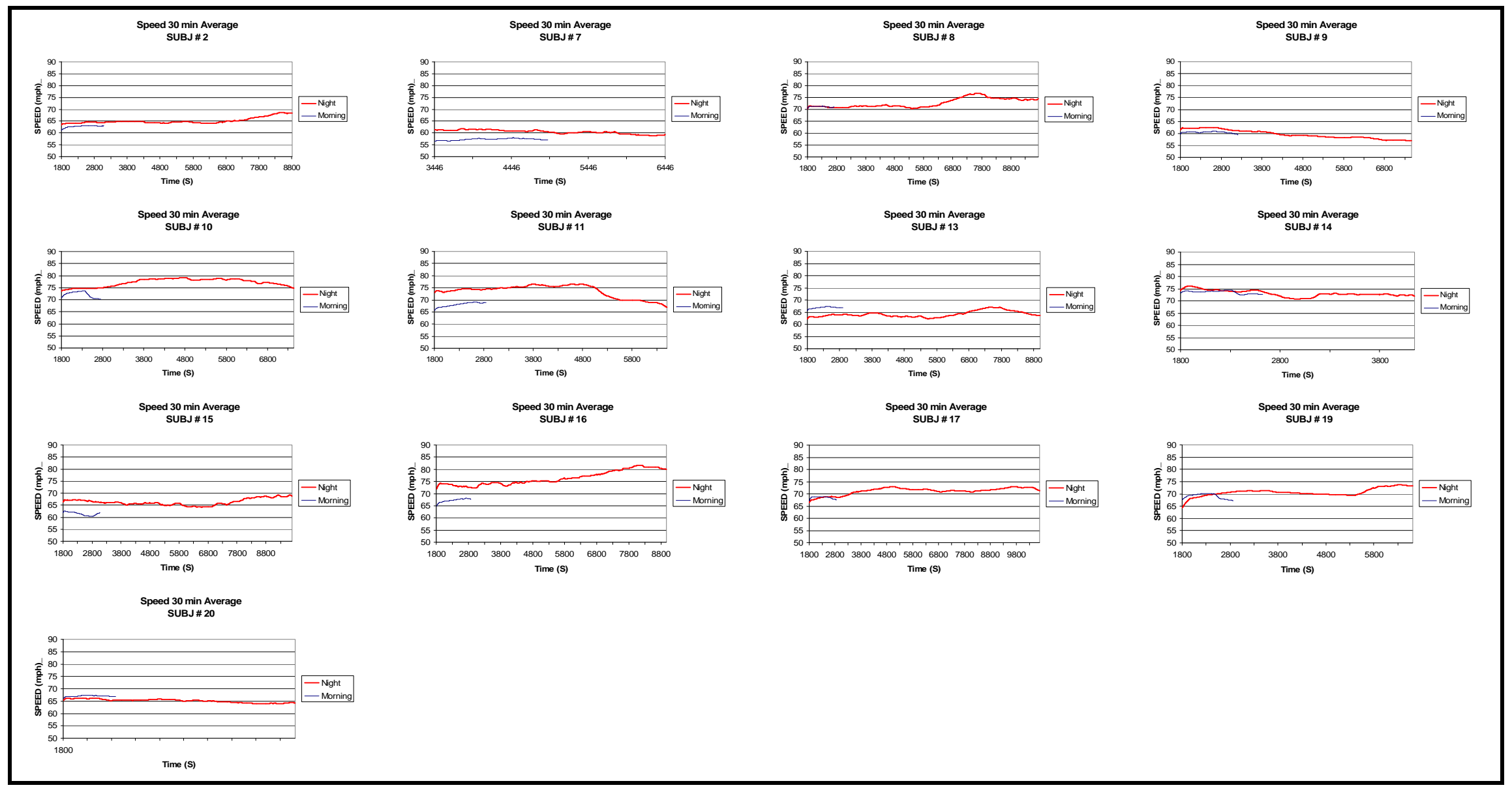

Figure 4.3: Speed 30-minute average for morning and night sessions 


\section{Gas/Throttle Pedal}

Because of the direct relation between speed and throttle opening, like vehicle speed, throttle opening cannot be used as a drowsiness indicator. The mean of throttle opening (GAS_MEAN as a percentage of full throttle) is shown in Table 4.5, and 30-minute average of throttle opening is shown in Figure 4.4. For 85\% of the drivers, both of these variables show a slight increase during the night sessions compared to the corresponding morning sessions but, like speed, this change did not arrive at a significant level. The standard deviation of the throttle opening (GAS_STDEV) showed no specific trends, as shown in Table 4.5. For most drivers, the 30-minute average of throttle opening follows a similar trend as the 30-minute average of speed because of the direct correlation between the gas pedal position and speed value.

Table 4.5: Throttle opening mean and standard deviation for morning and night sessions

\begin{tabular}{|c|c|c|c|c|}
\hline & \multicolumn{2}{|c|}{ GAS_MEAN (\% of full throttle) } & \multicolumn{2}{c|}{ GAS_STDEV (\% of full throttle) } \\
\hline SUBJ\# & Morning & Night & Morning & Night \\
\hline 2 & 47 & 48 & 12 & 10 \\
\hline 7 & 33 & 42 & 10 & 19 \\
\hline 8 & 50 & 59 & 9 & 14 \\
\hline 9 & 45 & 52 & 11 & 11 \\
\hline 10 & 57 & 64 & 12 & 11 \\
\hline 11 & 60 & 68 & 14 & 21 \\
\hline 13 & 51 & 47 & 10 & 7 \\
\hline 14 & 63 & 73 & 20 & 28 \\
\hline 15 & 47 & 51 & 14 & 20 \\
\hline 16 & 50 & 65 & 13 & 21 \\
\hline 17 & 58 & 72 & 10 & 15 \\
\hline 19 & 62 & 65 & 19 & 16 \\
\hline 20 & 50 & 48 & 14 & 12 \\
\hline Average & $\mathbf{5 1 . 8}$ & $\mathbf{5 8}$ & $\mathbf{1 3}$ & $\mathbf{1 5 . 8}$ \\
\hline
\end{tabular}




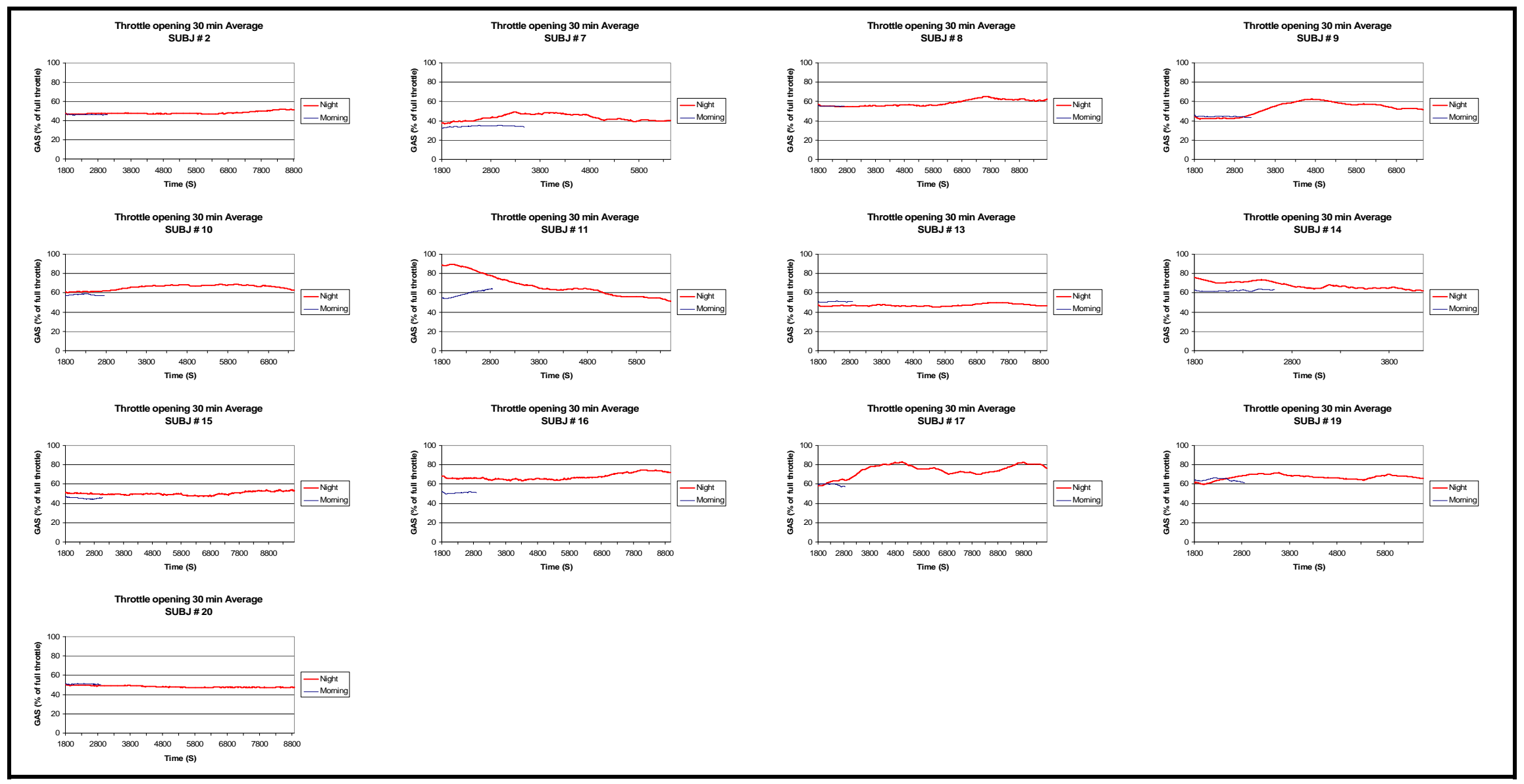

Figure 4.4: Throttle opening 30 -minute average for morning and night sessions 


\section{Vehicle Acceleration}

There is no significant change in the vehicle acceleration means (ACC_MEAN), (Table 4.6), and vehicle acceleration 30-minute averages (Figure 4.5) between morning and night sessions. This observation suggests no overall correlation between vehicle acceleration and driver drowsiness. Also, vehicle acceleration strongly depends on traffic and road geometry implying more challenging issues for removing these external effects to study the influence of drowsiness on vehicle acceleration.

Table 4.6: Vehicle acceleration mean and standard deviation for morning and night sessions

\begin{tabular}{|c|c|c|c|c|}
\hline & \multicolumn{2}{|c|}{ ACC_MEAN $\left(f t / s^{2}\right)$} & \multicolumn{2}{c|}{ ACC_STDEV } \\
\hline SUBJ\# & Morning & Night & Morning & Night \\
\hline 2 & 0.03 & 0.01 & 0.39 & 0.26 \\
\hline 7 & 0.20 & 0.13 & 0.39 & 0.92 \\
\hline 8 & 0.03 & 0.10 & 0.36 & 0.39 \\
\hline 9 & 0.03 & 0.01 & 0.30 & 0.23 \\
\hline 10 & 0.04 & 0.01 & 0.04 & 0.46 \\
\hline 11 & 0.04 & 0.04 & 0.59 & 0.52 \\
\hline 13 & 0.03 & 0.01 & 0.36 & 0.23 \\
\hline 14 & 0.04 & 0.04 & 0.95 & 1.31 \\
\hline 15 & 0.03 & 0.01 & 0.46 & 0.52 \\
\hline 16 & 0.03 & 0.01 & 0.36 & 0.75 \\
\hline 17 & 0.03 & 0.01 & 0.36 & 0.46 \\
\hline 19 & 0.03 & 0.02 & 0.56 & 0.33 \\
\hline 20 & 0.03 & 0.01 & 0.43 & 0.36 \\
\hline Average & 0.05 & 0.03 & 0.43 & 0.52 \\
\hline
\end{tabular}

\section{Braking Tap/Application}

The number of brake pedal applications (BRAKE_TAP) showed no significant variation in the alert and sleep deprived driving sessions. This variable had the value of zero for most of the time. Therefore, the number of brake applications will not provide useful information for a drowsiness detection algorithm.

\section{Crashes}

Four different variables with respect to crashes were analyzed as possible indicators of drowsiness: types of crashes, number of crashes, crash time, and crash point. Since crash prevention is the goal of a drowsiness detection/warning program, crashes would not be an appropriate input for such a system. However, crashes are useful for our data analysis because they can serve as a clear indicator of the onset of drowsiness. 


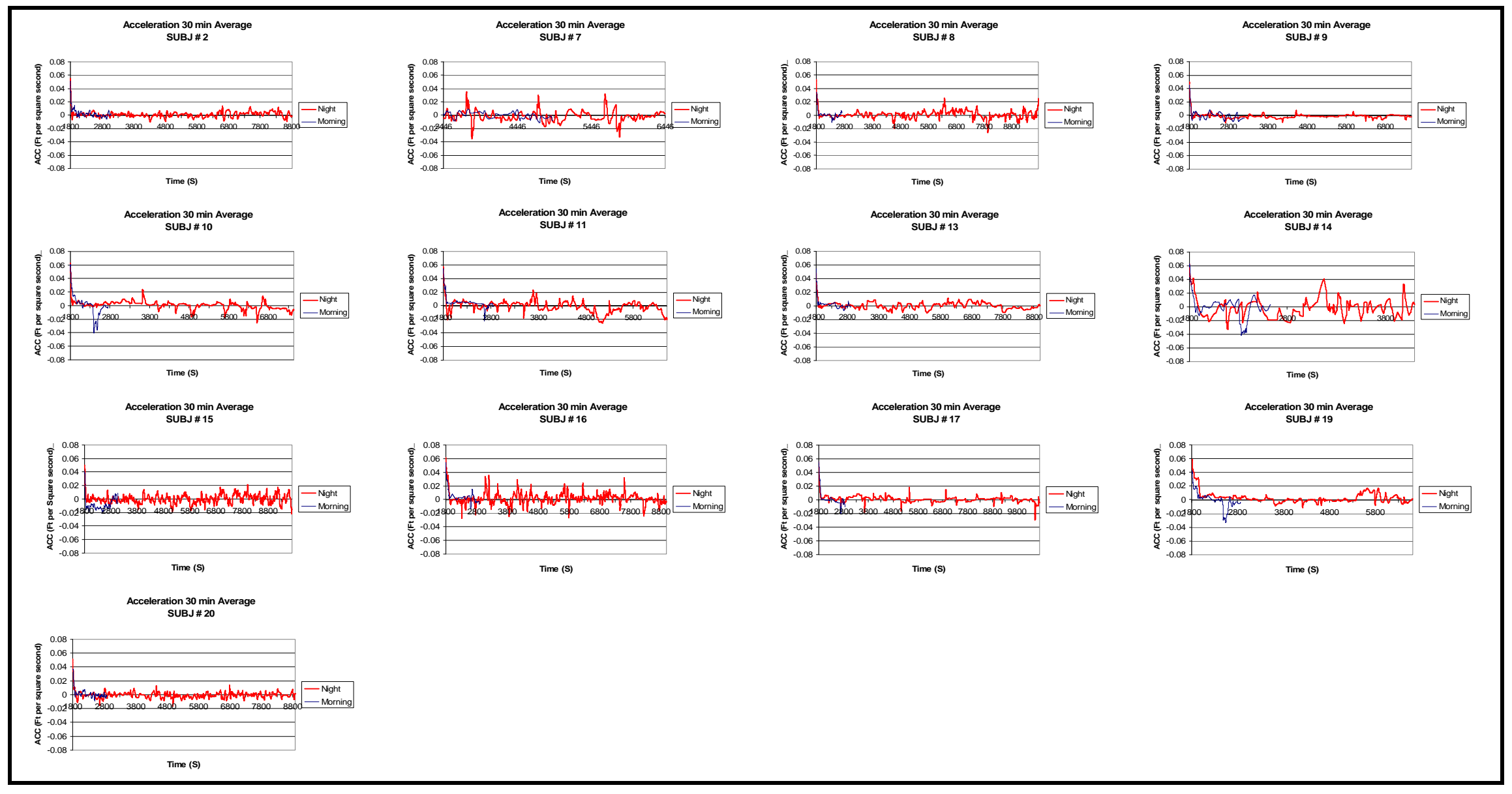

Figure 4.5: Acceleration 30-minute average for morning and night sessions 


\section{Types of Crashes}

Two types of crashes were defined in this project: run-off-road and collision crashes. According to test observations, eye data, and video recordings, drowsiness was the only cause of all the crashes during night sessions, which strongly implies the correlation between crashes and sleep deprivation. Ninety one percent of the night crashes were the result of run-off road incidents.

\section{Number of crashes}

Table 4.7 displays the number and side of the crashes during the night and morning sessions for each subject. As expected, the number of crashes heavily increased during the night sessions, especially for the drivers who experienced more doze-offs. There were very few drivers who experienced crashes during the morning session. These accidents were the result of driver inattention but not drowsiness.

Table 4.7: Number and type of crashes for morning and night sessions

\begin{tabular}{|c|c|c|c|c|c|c|c|}
\hline & \multicolumn{3}{|c|}{ Night } & \multicolumn{2}{c|}{ Morning } \\
\hline SUBJ\# & MDL & CRASH_RD_L & CRASH_RD_R & CRASH_V & Total & CRASH_RD & CRASH_V \\
\hline 2 & Right & 0 & 0 & 0 & 0 & 0 & 0 \\
\hline 7 & Right & 0 & 9 & 1 & 10 & 1 & 0 \\
\hline 8 & Left & 1 & 0 & 0 & 1 & 0 & 0 \\
\hline 9 & Right & 2 & 2 & 1 & 5 & 0 & 0 \\
\hline 10 & Left & 28 & 19 & 2 & 49 & 0 & 0 \\
\hline 11 & Right & 7 & 1 & 0 & 8 & 1 & 0 \\
\hline 13 & Right & 5 & 7 & 2 & 14 & 0 & 0 \\
\hline 14 & Left & 23 & 6 & 5 & 34 & 1 & 0 \\
\hline 15 & Right & 0 & 0 & 0 & 0 & 0 & 0 \\
\hline 16 & Right & 1 & 0 & 0 & 1 & 0 & 0 \\
\hline 17 & Right & 5 & 4 & 1 & 10 & 0 & 0 \\
\hline 19 & Left & 8 & 2 & 1 & 11 & 0 & 0 \\
\hline 20 & Right & 0 & 1 & 0 & 1 & 0 & 0 \\
\hline \multicolumn{2}{|l|}{ Average } & $\mathbf{6 . 1}$ & $\mathbf{4}$ & $\mathbf{1}$ & $\mathbf{1 1 . 1}$ & $\mathbf{0 . 2}$ & $\mathbf{0}$ \\
\hline
\end{tabular}

About $1 / 3$ of the drivers who mostly drove on the right lane had more left side run-offroad crashes (CRASH_RD_L). Similarly, the drivers with the preference of left lane driving had more left side run-off-road crashes (CRASH_RD_L) than right side run-off road crashes (CRASH_RD_R). Overall, more left side run-off-road crashes are experienced than right side run-off-road crashes.

\section{Crash time}

Figures 4.6 and 4.7 illustrate crash times for each subject. It should be noted that the design of the simulation scenario allowed the driver to continue driving after a crash without interrupting the simulation. According to the video data, after each crash, there was a significant increase in the driver's alertness level. Consequently, stopping the driving scenario after a crash might give the driver a chance to recover from drowsiness and delay the onset of the next sleep experience. The research team decided not to alert 
the drowsy drivers during their crashing maneuvers to collect more degraded driving performance data. Drivers were warned of their drowsiness by a buzzer installed inside the vehicle cabin behind their seat, when they reached the boundary of the threedimensional scene defined in the simulation software. Attempting to drive beyond this boundary could lead to computing error and simulation termination.

As a result, drowsy drivers sometimes crashed several times during one drowsiness event. Successive crashes could, therefore, occur in short time intervals. The minimum time between consecutive crashes of the same type was 13 seconds (subject 14). As shown by the eye and video data, during the intervals when there were concentrated numbers of crash incidents, the drivers showed more drowsy behaviors and experienced more dozeoffs. In addition, crash incidents were not uniformly distributed over time meaning periods of consecutive crash incidents proceeded by time intervals with no crash. This confirms the concept that drowsiness is not a continuous phenomenon and can occur sporadically.

\section{Crash point as an indicator of drowsiness}

Assuming that drowsiness was the cause of the accidents during night sessions, crash points can be used as a reliable reference for highlighting the drivers' drowsiness. Therefore, the first data ranges selected for data analysis were the intervals immediately before crashes. The performance of the drowsy driver detection system in these intervals is a key criterion for the evaluation of the system effectiveness. This analysis is provided in the next chapter.

\section{Drowsiness Effect on Steering Wheel Angle and Vehicle Lateral Position}

Driving can be described as a closed loop system in which the driver performs as a controller. During normal driving, the road and traffic information is perceived and analyzed by the driver, who sends a corrective command to the controlling devices, including the steering wheel, gas pedal and brake pedal, in order to keep the vehicle on the desired path and speed. Driving performance degradation due to drowsiness occurs in two consecutive phases. In the first phase, the so-called impaired phase, the driver decision making system is impaired, and the driver cannot smoothly control the vehicle, which in our analogy means that the controller is performing with a large error. The result is zigzag driving in which the driver cannot smoothly follow the desired trajectory. The effect of this phase is obvious in steering and lateral position data. During zigzag driving, vehicle lateral displacement and steering wheel angle standard deviation amounts increase with respect to normal driving values. This phenomenon may occur on and off before dozing off. In the next phase, the dozing off phase, the closed loop system becomes an open loop system causing the vehicle to continue its path without any correction as a result of the driver's dozing off. This can be traced by constant steering 


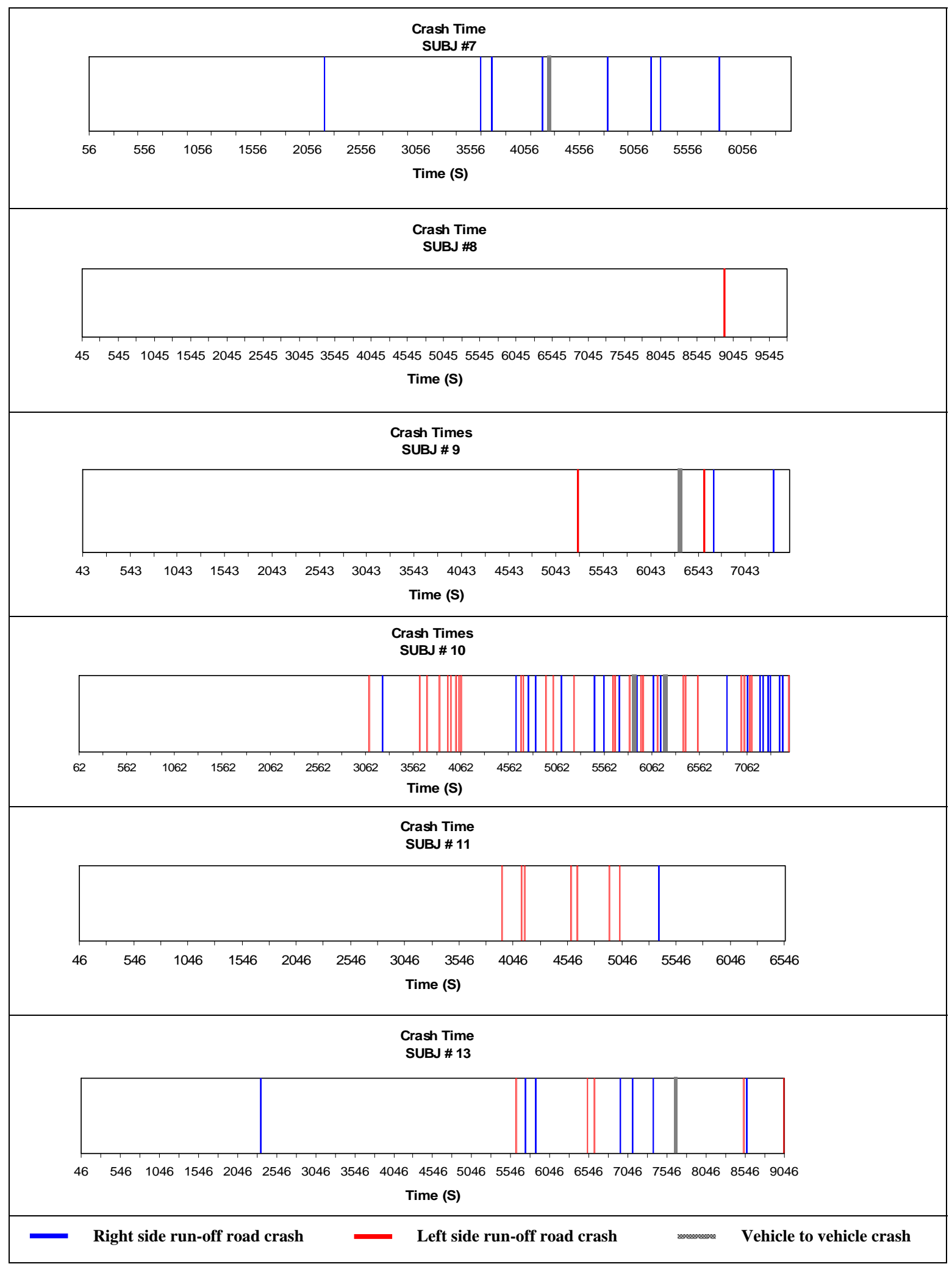

Figure 4.6: Crash times for night sessions (Subjects 7-13) 


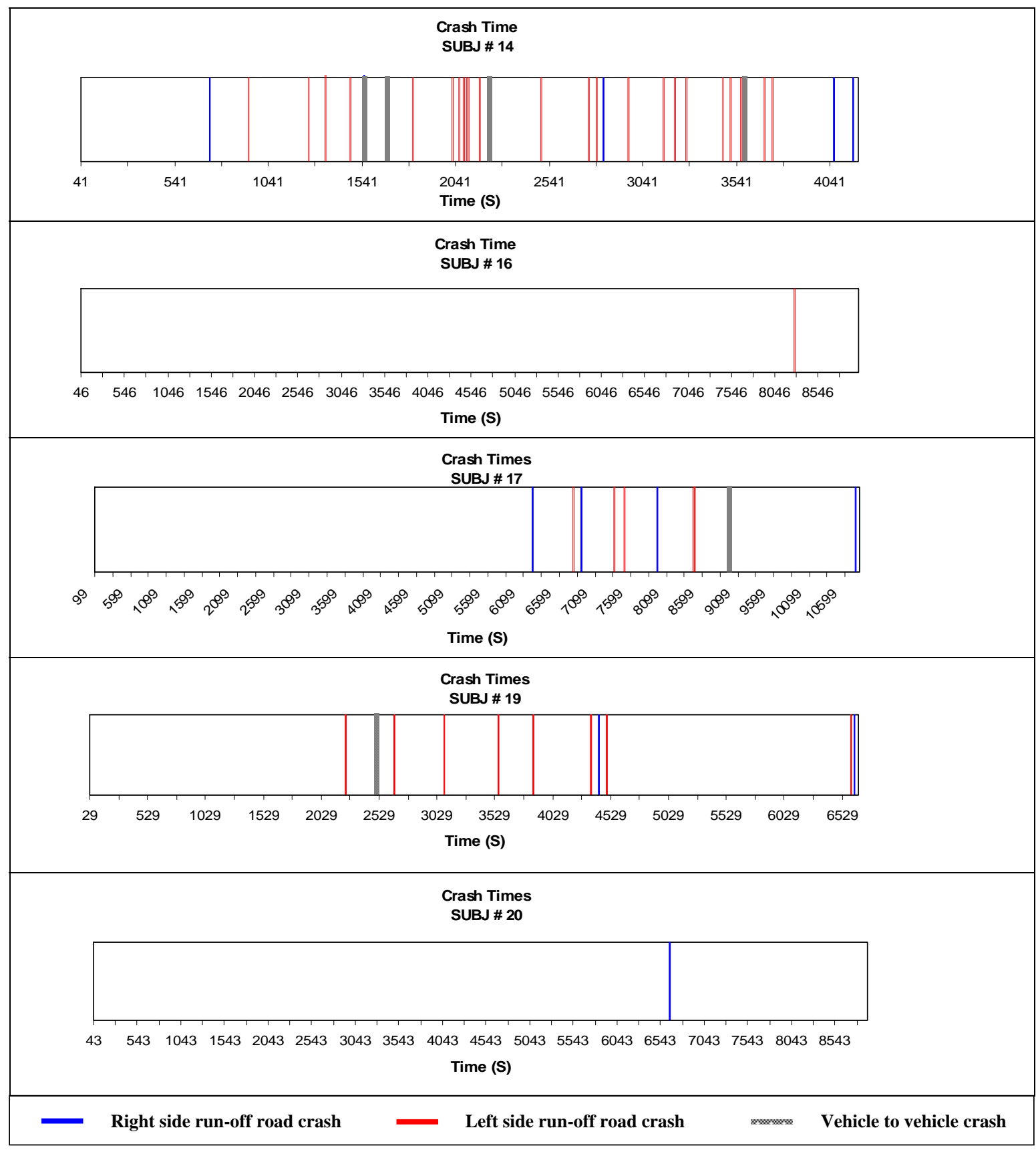

Figure 4.7: Crash times for night sessions (Subjects 14-20) 

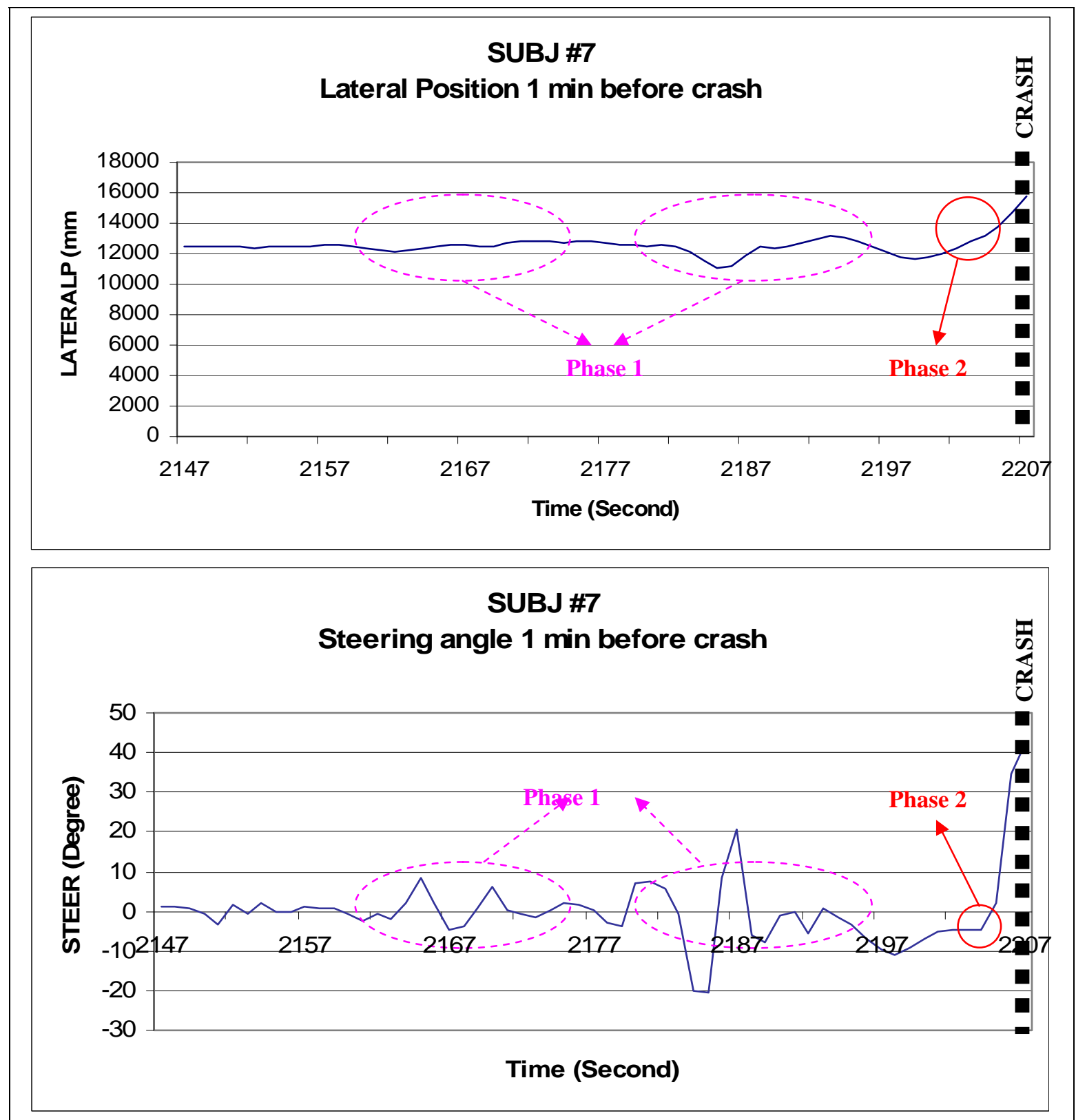

Figure 4.8: Vehicle lateral position and steering angle one minute before crash

values over a short period of time and increasing values in lateral displacement data. Most vehicle drifts and run-off road crashes occur during this phase. In some occasions, drivers who dozed off were lucky enough to continue driving on a straight path without any drift or run-off-road crash. This event is more likely to happen in the simulation environment than in real world driving because of the road cross slope, which can make a vehicle drift to the side, is not modeled in the simulation. Figure 4.8 displays the described signs of driving performance degradation in the lateral position and steering data one minute before one of the subject's first crash. 


\section{Lateral Displacement}

Lateral displacement is defined as the absolute normal distance between the vehicle center of gravity and the current lane's centerline. As previously described, the drivers were allowed to continue driving without any interruption after each crash. This created unrealistic values of lateral displacement after run-off-road crashes affecting the data analysis results. To avoid that, these values were replaced by the value of vehicle's lateral displacement at the time of the crash.

The lateral displacement mean and standard deviation values for morning and night sessions are listed in Table 4.8. All the subjects demonstrated significantly higher lateral displacement means (LATERALD_MEAN) and standard deviations (LATERALD_STDEV) for night sessions, which confirms our observations about the drowsiness effect on the vehicle's lateral position.

Table 4.8: Lateral displacement mean and standard deviation during morning and night sessions

\begin{tabular}{|c|c|c|c|c|c|c|}
\hline & \multicolumn{2}{|c|}{ LATERALD_MEAN (ft) } & \multicolumn{3}{c|}{ LATERALD_STDEV } \\
\hline SUBJ\# & Morning & Night & $\begin{array}{c}\text { \% } \\
\text { increase }\end{array}$ & Morning & Night & $\begin{array}{c}\text { \% } \\
\text { increase }\end{array}$ \\
\hline 2 & 0.8 & 1.2 & 35 & 0.7 & 1.0 & 29 \\
\hline 7 & 1.3 & 1.9 & 30 & 0.9 & 1.6 & 42 \\
\hline 8 & 1.4 & 1.6 & 9 & 1.0 & 1.1 & 10 \\
\hline 9 & 1.1 & 1.5 & 29 & 0.9 & 1.4 & 39 \\
\hline 10 & 1.3 & 2.4 & 45 & 1.0 & 2.9 & 66 \\
\hline 11 & 1.3 & 2.0 & 34 & 1.0 & 1.5 & 33 \\
\hline 13 & 1.1 & 1.3 & 20 & 0.9 & 1.6 & 44 \\
\hline 14 & 1.7 & 2.8 & 40 & 1.1 & 2.5 & 56 \\
\hline 15 & 1.6 & 1.7 & 10 & 0.8 & 0.8 & 3 \\
\hline 16 & 1.3 & 1.7 & 24 & 0.9 & 1.2 & 29 \\
\hline 17 & 0.9 & 1.5 & 38 & 0.9 & 1.2 & 21 \\
\hline 19 & 1.5 & 1.8 & 18 & 1.1 & 1.6 & 34 \\
\hline 20 & 1.0 & 1.3 & 25 & 0.8 & 1.2 & 38 \\
\hline Average & $\mathbf{1 . 2}$ & $\mathbf{1 . 7}$ & $\mathbf{2 9}$ & $\mathbf{0 . 9}$ & $\mathbf{1 . 5}$ & $\mathbf{4 0}$ \\
\hline
\end{tabular}

The 30-minute average and 30-minute standard deviation graphs of the lateral displacement (Figures 4.9 and 4.10) show higher values for a majority of the night sessions compared to the morning sessions. These graphs, therefore, indicate that the drivers experienced both higher average deviations and more variability in their lane keeping results during night driving than during morning driving. Moreover, lateral displacement standard deviation values for night sessions vary while the morning values are almost constant for most drivers. This variation suggests that drivers' ability to control the lateral position of their vehicle in the traveling lane degrades during the night session. Drowsiness not only increases the average lane deviation but also the variability of drivers' lane keeping performance. 


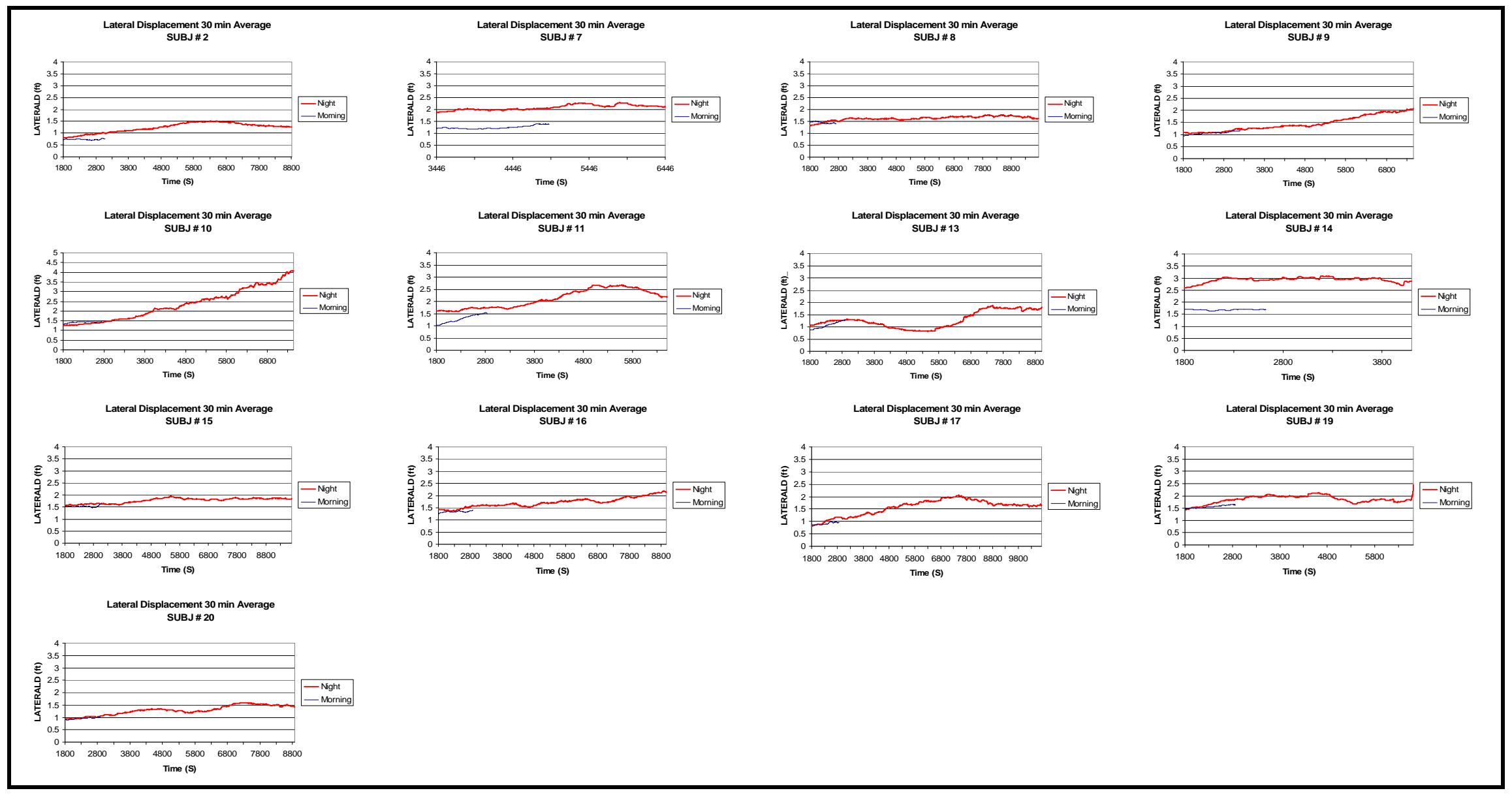

Figure 4.9: Lateral displacement 30-minute average for morning and night sessions 


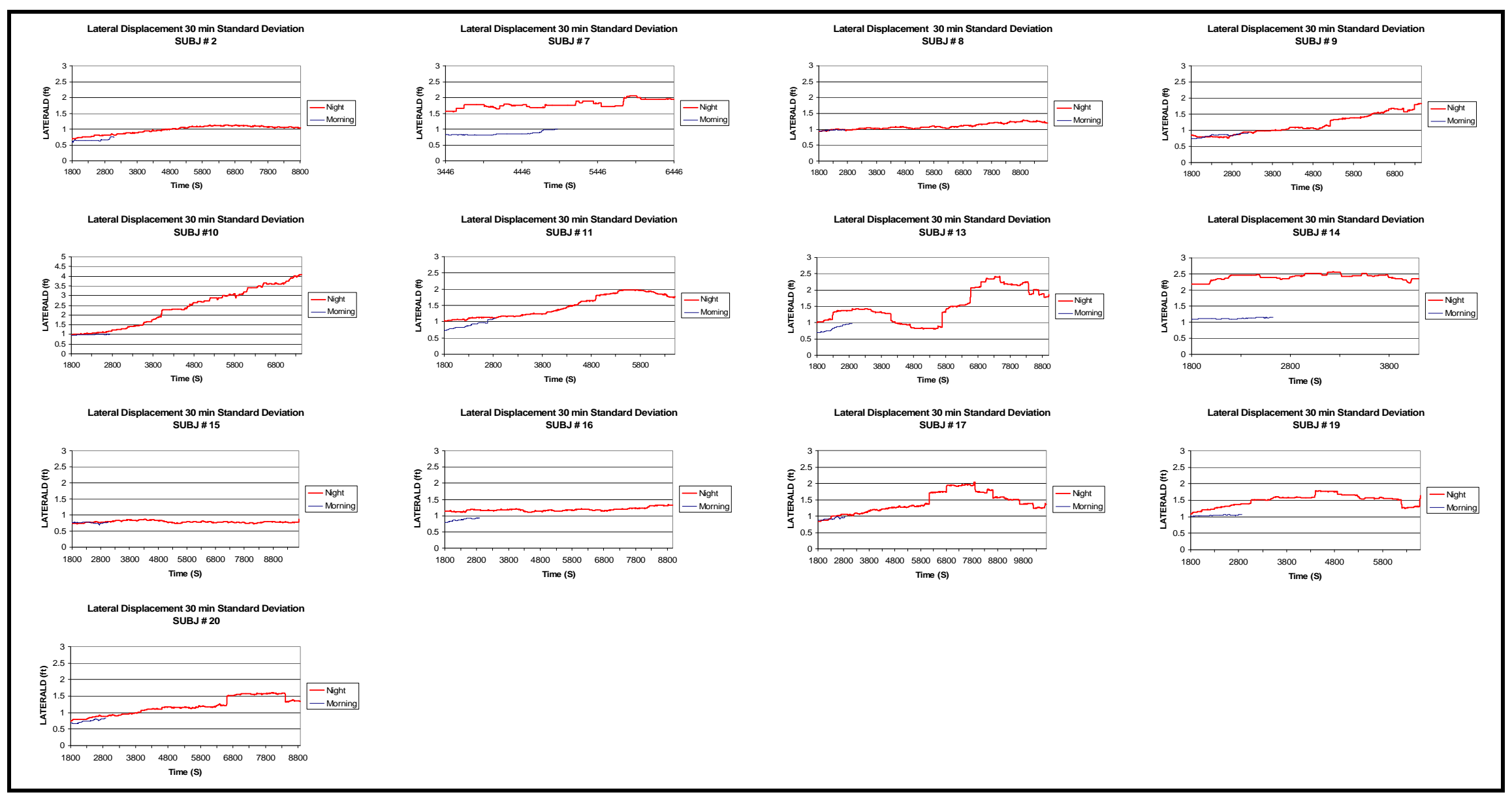

Figure 4.10: Lateral displacement 30-minute standard deviation for morning and night sessions 
The morning (normal) lateral displacement average varies among the drivers due to different individual driving styles. Some people have a higher average lateral displacement during their normal driving. Nonetheless, this almost constant lateral displacement curve for morning driving is assumed as a baseline for alert or normal driving performance.

Figure 4.11 displays PERCLOS and lateral displacement 30 min average and standard deviation for a sample of subjects to help the reader easily compare the graphs. This comparison illustrates that there is no direct one to one correlation with the PERCLOS data. However, when a subject's lateral displacement standard deviation curve has an increasing trend with respect to the corresponding morning curve, the PERCLOS 30-min average graph also displays higher values comparing to its alert baseline.

To have a better understanding of lateral displacement behavior while drivers are drowsy, one minute histograms of the lateral displacement during the 5 minutes before crash is illustrated in Figure 4.12 for approximately $10 \%$ of the total of crashes (12 crashes). The five minute interval before a crash is chosen in order to guarantee the prevalence of a driver impaired phase. Similar histograms randomly chosen from five minutes of morning driving data are also plotted in Figure 4.12 to show an example of lateral displacement variation under normal (alert) conditions. Histograms from morning sessions are not meant to be directly compared to histograms from night sessions, but can provide insight into normal baseline driving data.

In each one-minute data window, mean, standard deviation, and median of the steering signals are depicted. The x-axis shows lateral displacement bins (intervals). The number of lateral displacement values that fall into each bin range is counted and displayed as a bar.

These graphs indicate the following:

- The night session histograms show a higher incidence of large lateral displacement compared to the morning sessions.

- The night sessions had higher mean and standard deviation values than the morning baseline.

- $\quad 92 \%$ of the analyzed crash data had higher standard deviation values during the minute intervals immediately preceding the crash, when compared to other night session intervals. 


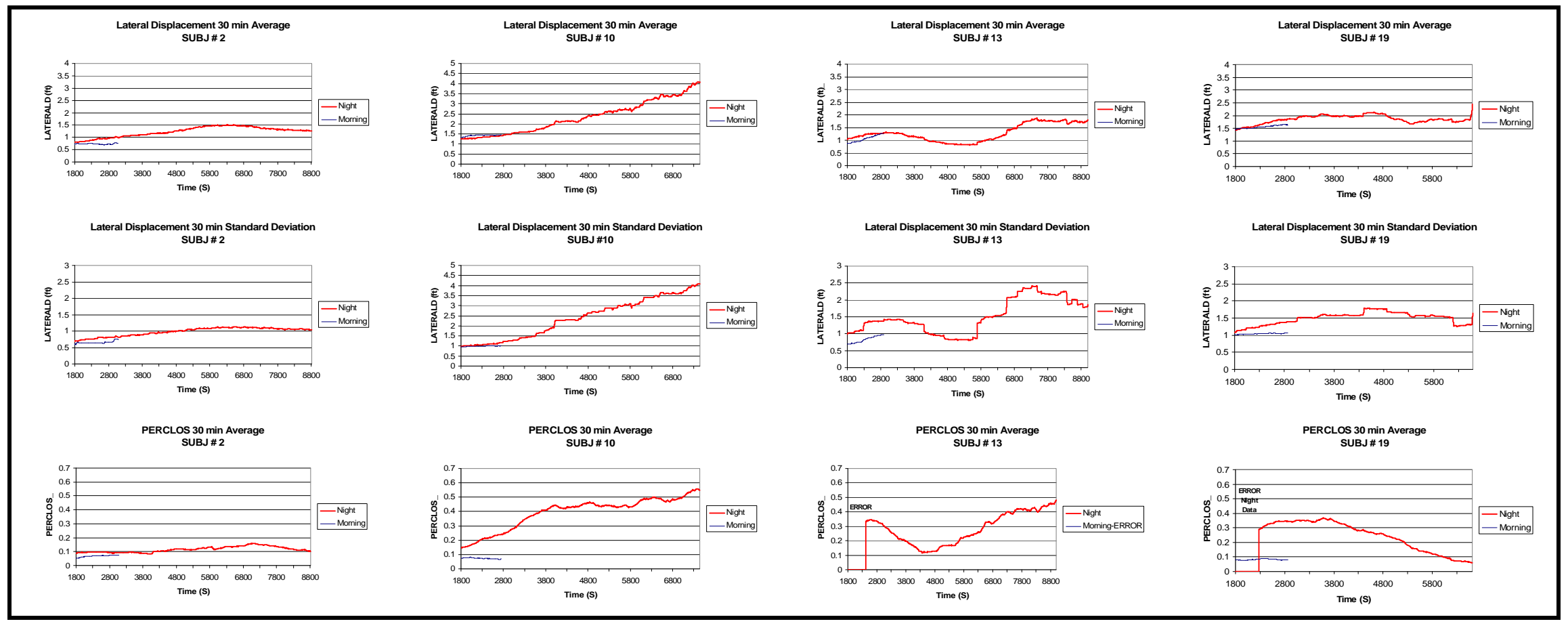

Figure 4.11: Comparison of lateral displacement graphs and PERCLOS 
SUBJECT \# 7

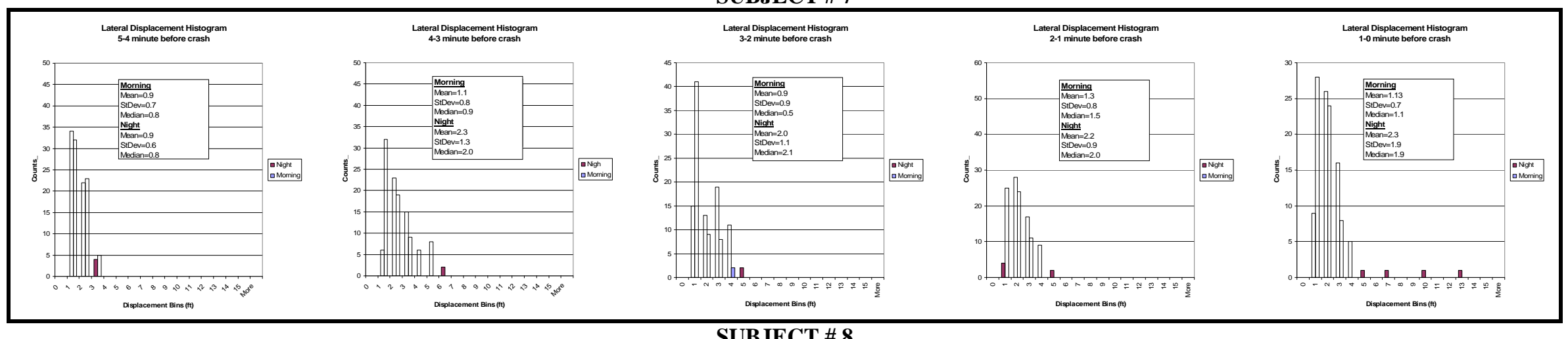

SUBJECT \# 8

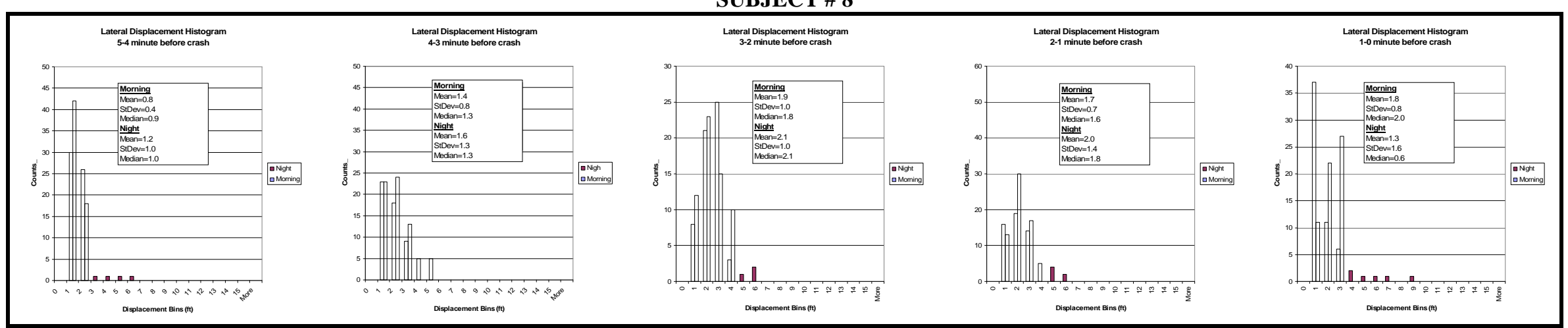

SUBJECT \# 9

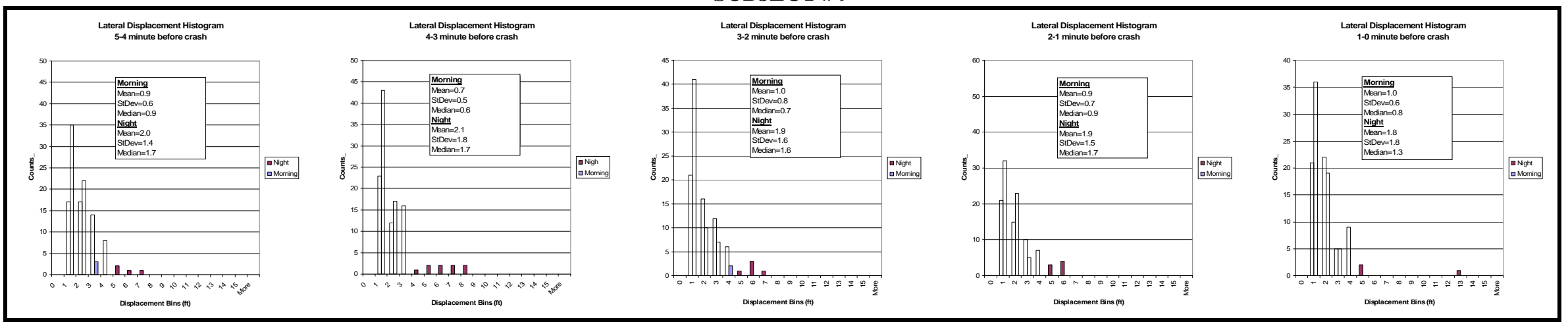

Figure 4.12: Lateral displacement signal and lateral displacement power 1 to 5 minutes before a crash compared to baseline data from morning sessions 
SUBJECT \# 10

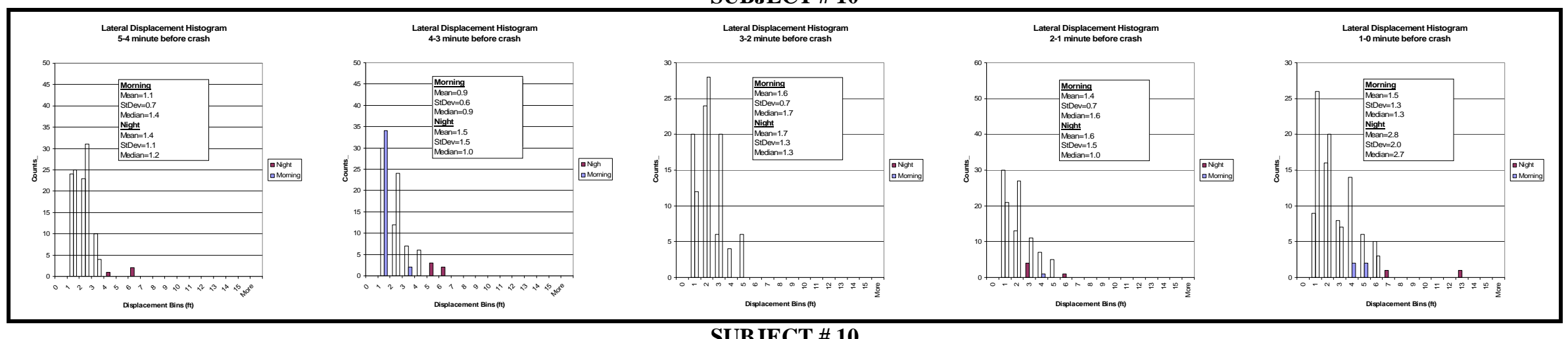

SUBJECT \# 10

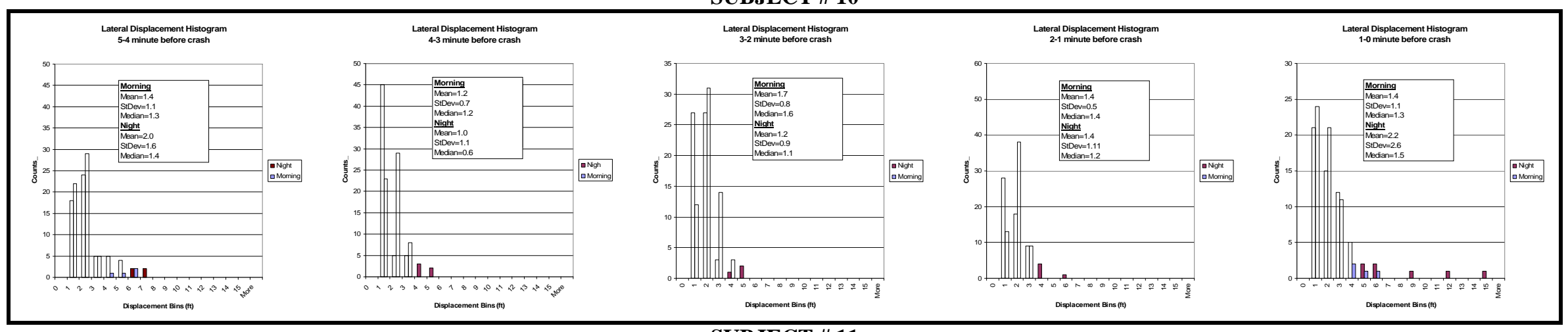

SUBJECT \# 11

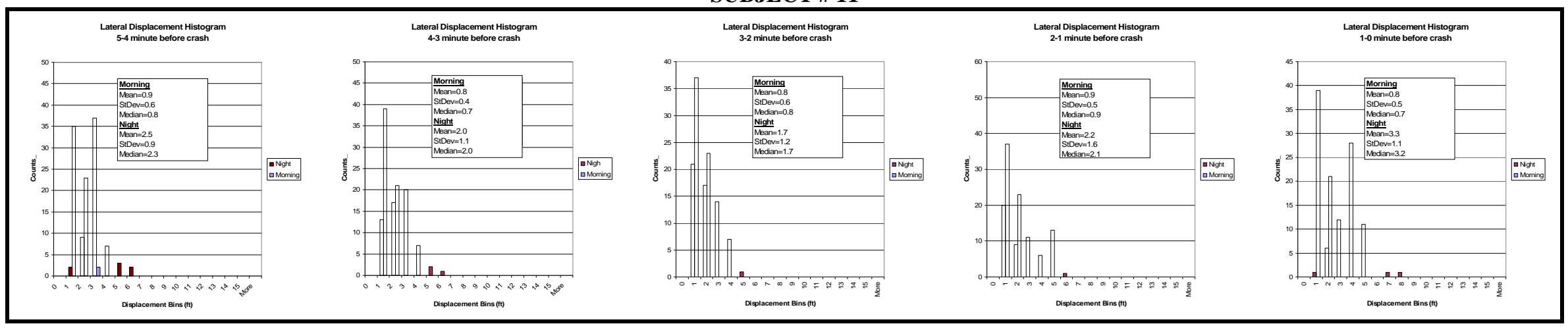

Figure 4.12 (continued) 
SUBJECT \# 13
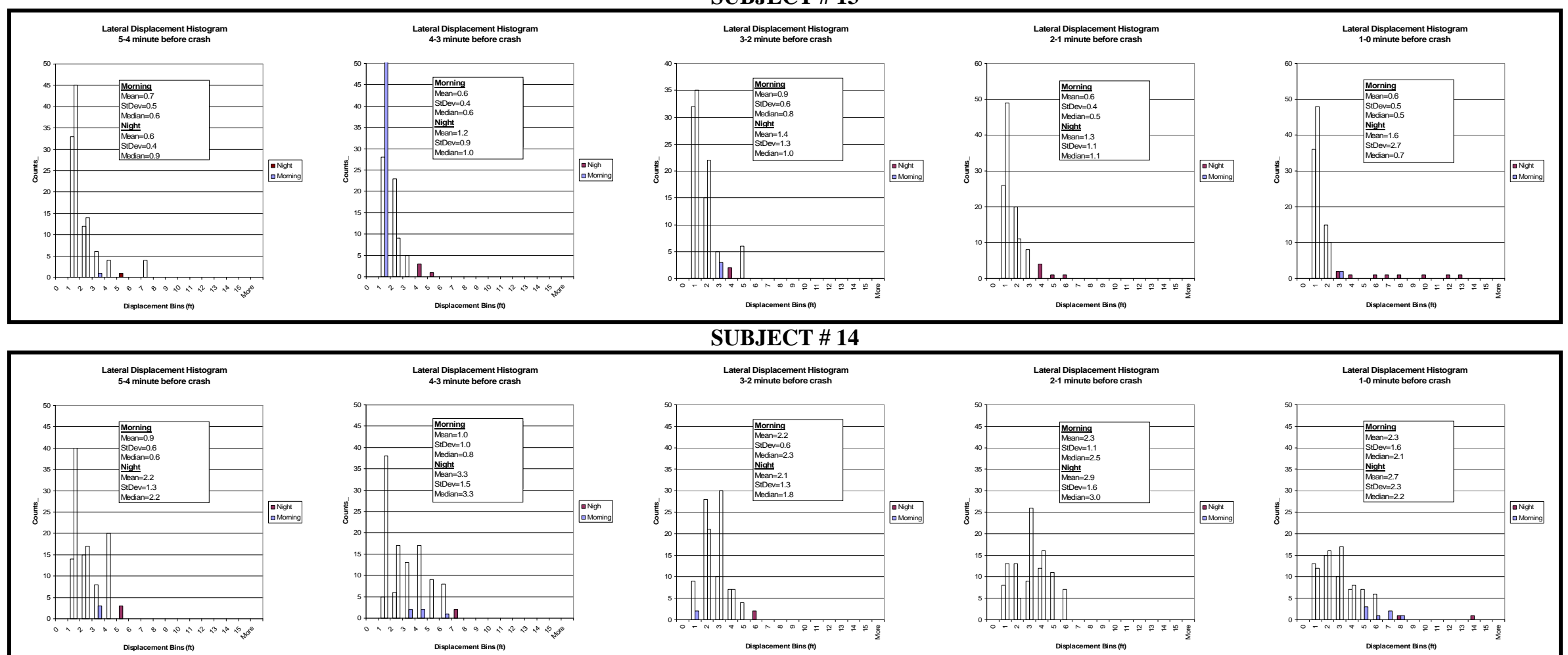

Lateral Displacement tistogram
2-1 minute betore crash

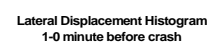

SUBJECT \# 15
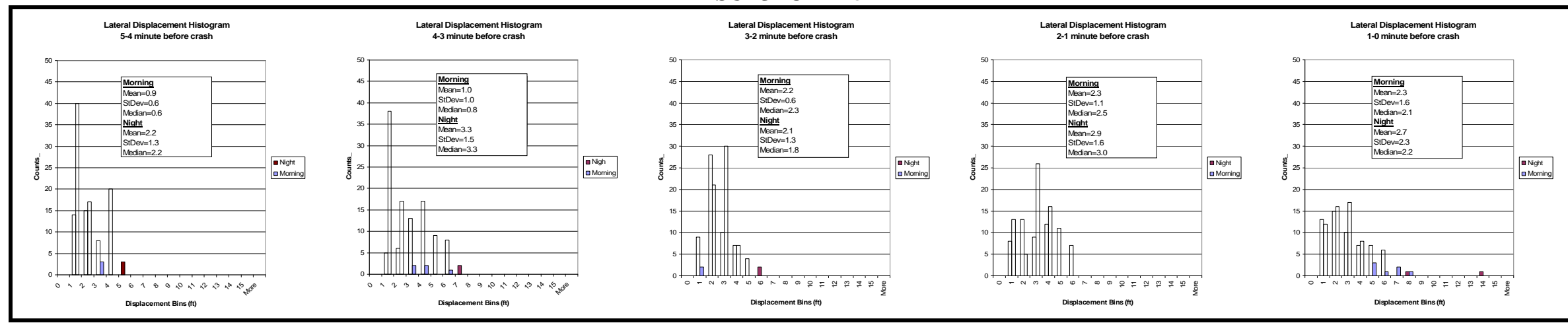

Figure 4.12 (continued) 
SUBJECT \# 16

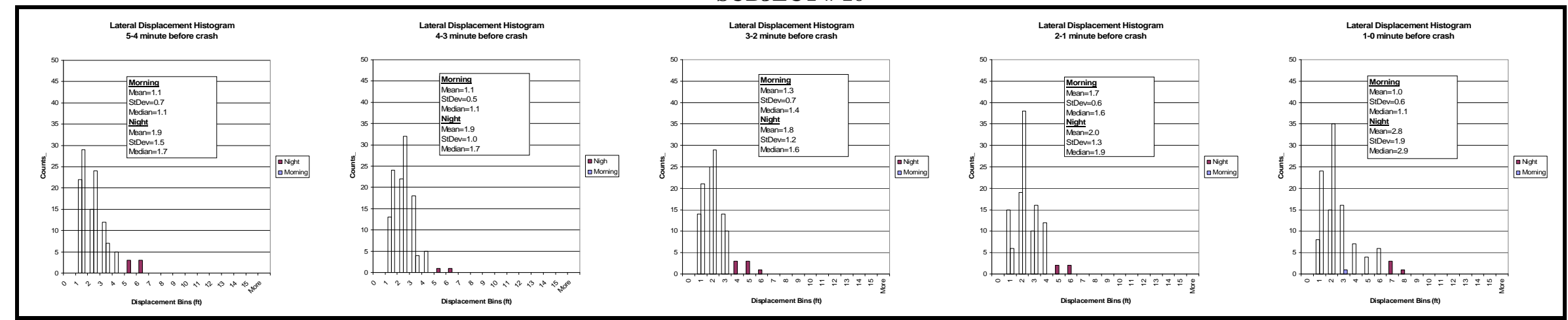

SUBJECT \# 17
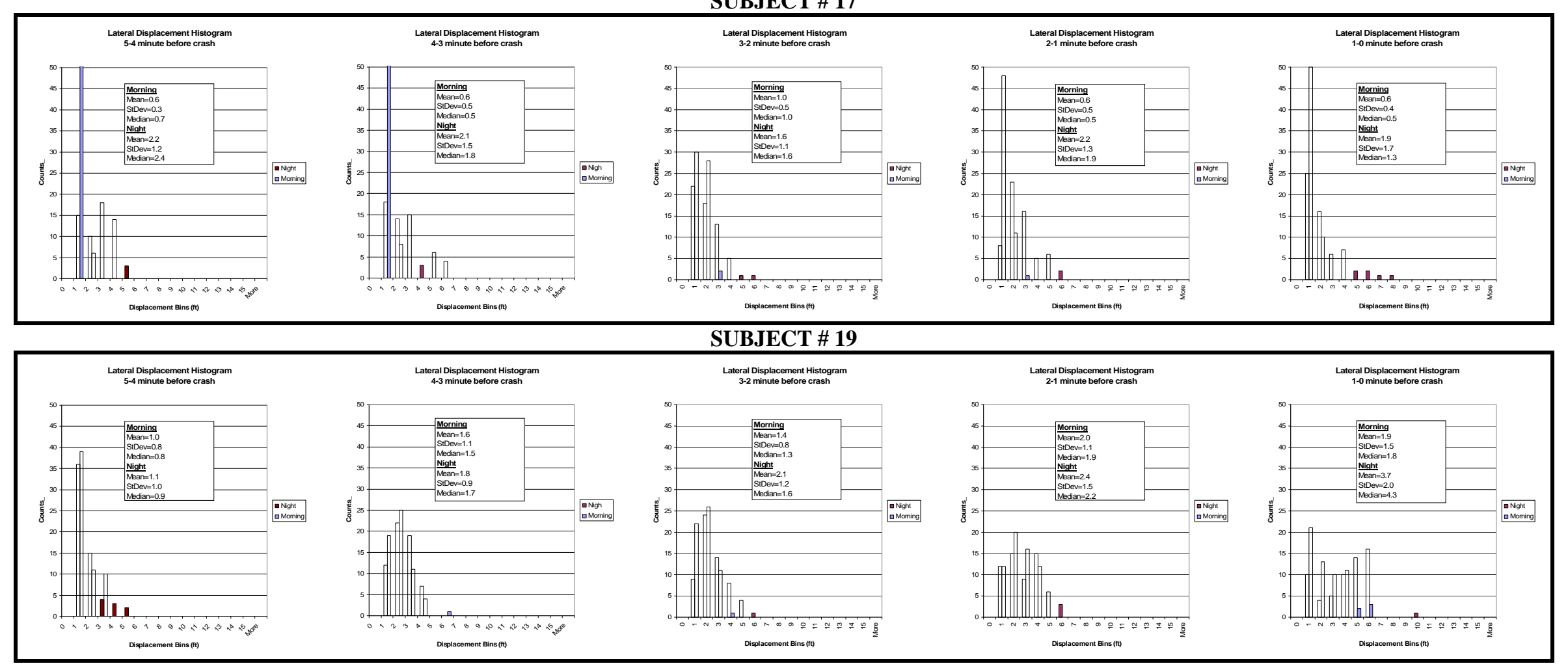

Figure 4.12 (continued) 
The results show that lateral displacement variability is related to the amount of drowsiness in the subjects suggesting that it can be used in as a good measure in a drowsy driver detection system.

Another way of examining a driver's performance and responsiveness is to study the number of marking line crossings (left, center, and right lines) and the total time that the vehicle was driven on or beyond the marking lines. The simulated roadway was a four lane, divided highway with continuous left and right shoulder marking lines and dashed center line. Line crossing occurs when one of the vehicle tires touches the shoulder line. The number of center line crossings is defined as the number of times that the vehicle crossed the dashed line without using the turning signals. Table 4.9 displays the values for left, center, and right line crossings, as well as the lane mostly driven by each subject.

All the drivers who drove mostly on the left lane had more left line crossings than right line crossings. For right lane drivers, left and right line crossings were more balanced, with approximately the same number of left line crossings (45\%) than right line crossings (55\%). Most of left line crossings occurred when passing a vehicle on the left.

\section{Number of Lane Changes}

Figure 4.13 displays the number of lane change (N_LANE_CH) per 30 minutes during morning and night sessions for different subjects. Generally, N_LANE_CH values increase over the periods that the drivers feel drowsy. This variable shows good correlation with the sleep deprivation. However, the variability of this parameter is not significant for short time intervals. Therefore, it cannot be used as an indicator in the drowsiness detection system. 
Table 4.9: Time the vehicle was driven beyond marking lines and number of line crossings during morning and night sessions (bold values represent night sessions)

\begin{tabular}{|c|c|c|c|c|c|c|c|c|c|}
\hline \multirow[b]{2}{*}{ SUBJ\# } & \multicolumn{4}{|c|}{$\begin{array}{l}\text { TOTAL DRIVING TIME ON OR BEYOND MARKING } \\
\text { LINE(Second) }\end{array}$} & \multicolumn{4}{|c|}{ TOTAL NUMBER OF CROSSINGS } & \multirow{2}{*}{$\begin{array}{c}\text { LANE } \\
\text { MOSTLY } \\
\text { DRIVEN } \\
\end{array}$} \\
\hline & T_CROSS_L & T_CROSS_C & T_CROSS_R & TOTAL & N_CROSS_L & N_CROSS_C & N_CROSS_R & TOTAL & \\
\hline 2 & $36 / 142$ & $57 / 770$ & $18 / 268$ & $111 / 1180$ & $3 / 22$ & $5 / 63$ & $2 / 44$ & $10 / 129$ & Right \\
\hline 7 & $0 / 1$ & $19 / 133$ & $630 / 2010$ & $649 / 2144$ & $0 / 1$ & $6 / 29$ & $7 / 31$ & 13 / 61 & Right \\
\hline 8 & $367 / 1082$ & $198 / 1384$ & $7 / 51$ & $572 / 2517$ & $8 / 49$ & $9 / 62$ & $2 / 15$ & $19 / 126$ & Left \\
\hline 9 & $45 / 195$ & $117 / 551$ & $141 / 858$ & $303 / \mathbf{1 6 0 7}$ & $3 / 16$ & $16 / 89$ & $16 / 84$ & 35 / 189 & Right \\
\hline 10 & $141 / 966$ & $227 / 884$ & $8 / 622$ & $376 / 2472$ & $14 / 75$ & $16 / 110$ & $3 / 40$ & $33 / 225$ & Left \\
\hline 11 & 294 / 1089 & 187 / 1296 & $0 / 108$ & $481 / 2493$ & $6 / 44$ & $7 / 57$ & $0 / 13$ & $13 / 114$ & Right \\
\hline 13 & $36 / 150$ & $231 / 788$ & $31 / 425$ & $298 / 1363$ & $5 / 26$ & $10 / 70$ & $5 / 41$ & $20 / 137$ & Right \\
\hline 14 & $478 / 1211$ & $208 / 659$ & $1 / 211$ & 687 / 2081 & $17 / 52$ & $19 / 74$ & $1 / 23$ & $37 / 149$ & Left \\
\hline 15 & 185 / 1047 & 449 / 1369 & $0 / 4$ & 634 / 2420 & $6 / 33$ & $7 / 35$ & $0 / 1$ & 13 / 69 & Right \\
\hline 16 & 87 / 886 & $281 / 1672$ & $2 / 28$ & $370 / 2586$ & $6 / 73$ & $8 / 81$ & $1 / 7$ & $15 / 161$ & Right \\
\hline 17 & $221 / 1464$ & $15 / 511$ & $1 / 270$ & $237 / 2245$ & $5 / 49$ & $5 / 72$ & $1 / 40$ & $11 / \mathbf{1 6 1}$ & Right \\
\hline 19 & 244 / 988 & $342 / 1053$ & $18 / 119$ & $604 / 2160$ & $11 / 49$ & $16 / 69$ & $5 / 26$ & $32 / 144$ & Left \\
\hline 20 & $70 / 123$ & $27 / 770$ & $2 / 298$ & 99 / 1191 & $6 / 22$ & $7 / 67$ & $1 / 46$ & 14 / 135 & Right \\
\hline Average & $169.5 / 718.8$ & 181.4 / 910.8 & $66.1 / 405.5$ & $417 / 2035$ & 6.9 / 39.3 & $10.1 / 67.5$ & $3.4 / 31.6$ & $20.4 / 138.5$ & Right \\
\hline
\end{tabular}




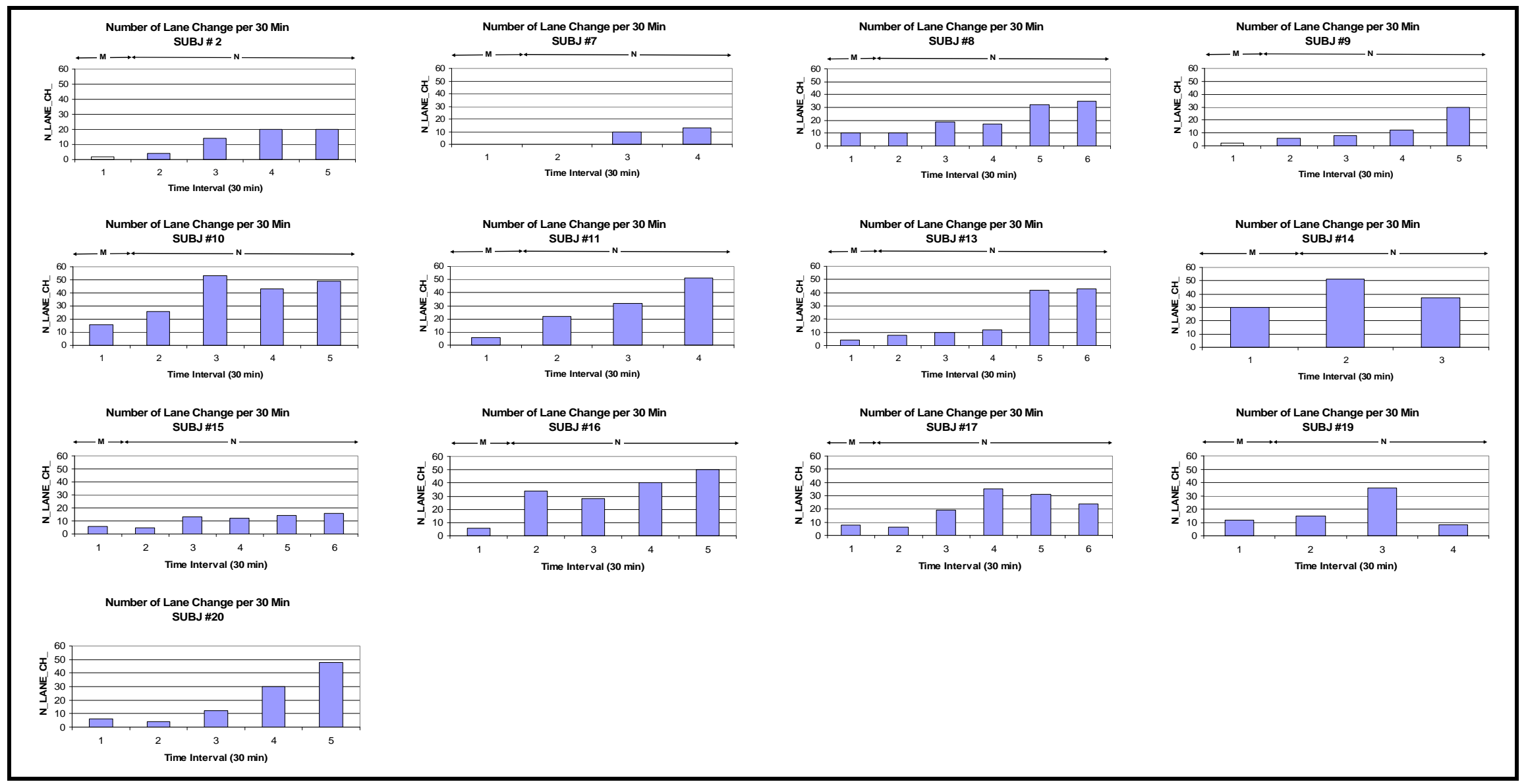

Figure 4.13: Number of lane change per 30 minutes during Morning $(M)$ and Night $(N)$ sessions for each subject 


\section{Heading Angle}

Table 4.10 shows vehicle heading angle mean (HEADING_MEAN) and standard deviation (HEAD_STDEV) values for each subject during the night and morning sessions. Figure 4.14 also displays heading angle (HEAD) 30-minute average graphs. Given that lateral position is related to the vehicle heading angle, the conclusions made for the lateral position are also applicable to the heading angle.

Table 4.10: Vehicle heading angle mean and standard deviation for morning and night sessions

\begin{tabular}{|c|c|c|c|c|}
\hline & \multicolumn{2}{|c|}{ HEAD_MEAN (Degree) } & \multicolumn{2}{c|}{ HEAD_STDEV } \\
\hline SUBJ\# & Morning & Night & Morning & Night \\
\hline 2 & 9 & 11 & 10 & 14 \\
\hline 7 & 18 & 21 & 34 & 27 \\
\hline 8 & 9 & 11 & 11 & 14 \\
\hline 9 & 13 & 19 & 13 & 24 \\
\hline 10 & 11 & 19 & 16 & 46 \\
\hline 11 & 7 & 14 & 11 & 19 \\
\hline 13 & 13 & 18 & 14 & 29 \\
\hline 14 & 13 & 26 & 18 & 26 \\
\hline 15 & 7 & 9 & 10 & 13 \\
\hline 16 & 9 & 14 & 11 & 19 \\
\hline 17 & 8 & 17 & 11 & 19 \\
\hline 19 & 11 & 15 & 13 & 21 \\
\hline 20 & 9 & 15 & 10 & 17 \\
\hline Average & 10 & 16 & 14 & 22 \\
\hline
\end{tabular}




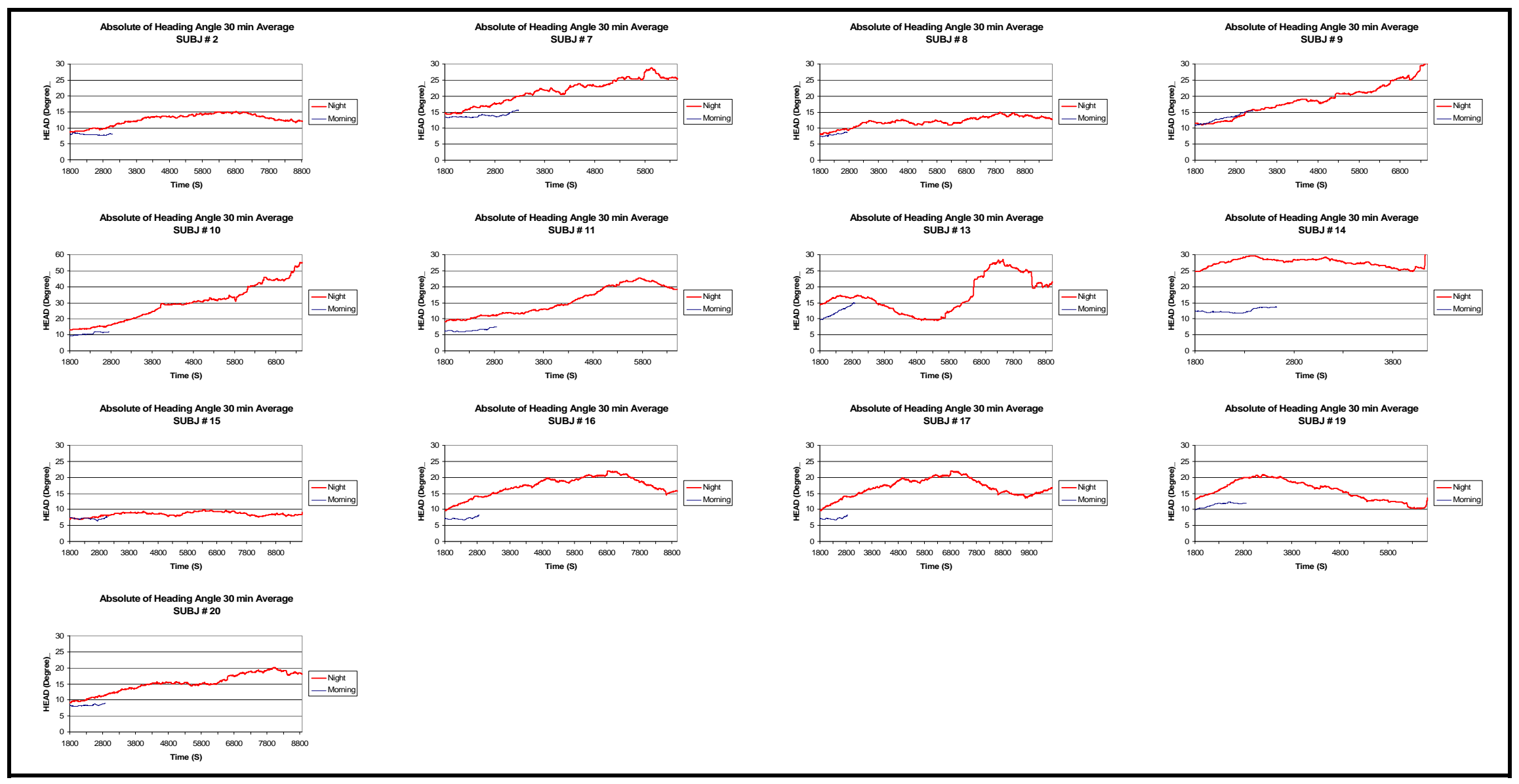

Figure 4.14: Vehicle heading angle 30-minute average for morning and night sessions 


\section{Steering Wheel Angle}

Since the steering angle data contains positive and negative values, its mean and standard deviation does not demonstrate any trend. Thus, the steering angle instantaneous power, defined as the steering angle squared, is used to analyze the effect of drowsiness on the steering performance. Using steering signal power for analysis provides two advantages over using the signal absolute value. First, it represents a meaningful physical characteristic of the steering angle signal. Second, it amplifies the large steering corrections associated with driver drowsiness and, therefore, increases the difference between alert and drowsy steering results.

In curved sections of the roadway, drivers' steering wheel inputs do not only correct for vehicle lateral deviations but also for the road curvature. The portion of steering angle intended to compensate for road curvature was subtracted from the original steering angle data. The process of eliminating road curvature is discussed in the section on Effect of Road Curvature in Chapter 5. Table 4.11 shows steering angle power mean (STEER_P_MEAN) and standard deviation (STEER_P_STDEV) for each subject during the morning and night sessions. This table shows that during the night sessions, the steering angle power mean and standard deviation have higher values when compared to morning values.

Table 4.11: Steering angle power mean and standard deviation for morning and night sessions

\begin{tabular}{|c|c|c|c|c|c|c|}
\hline & \multicolumn{2}{|c|}{ STEER_P_MEAN (Degree ${ }^{2}$ ) } & \multicolumn{2}{c|}{ STEER_P_STDEV (Degree $\left.{ }^{2}\right)$} \\
\hline SUBJ\# & Morning & Night & $\begin{array}{c}\mathbf{\%} \\
\text { increase }\end{array}$ & Morning & Night & $\begin{array}{c}\text { \% } \\
\text { increase }\end{array}$ \\
\hline 2 & 6.4 & 9.1 & 30 & 25.0 & 30.1 & 20 \\
\hline 7 & 22.5 & 55.9 & 60 & 191.0 & 179.8 & $-10^{*}$ \\
\hline 8 & 4.9 & 8.6 & 40 & 24.4 & 43.0 & 40 \\
\hline 9 & 6.5 & 20.8 & 70 & 20.1 & 91.4 & 80 \\
\hline 10 & 7.3 & 41.2 & 80 & 26.5 & 152.1 & 80 \\
\hline 11 & 3.3 & 18.0 & 80 & 15.9 & 67.5 & 80 \\
\hline 13 & 7.6 & 14.9 & 50 & 27.6 & 61.1 & 50 \\
\hline 14 & 5.8 & 26.6 & 80 & 21.2 & 97.7 & 80 \\
\hline 15 & 4.1 & 6.2 & 30 & 12.2 & 18.8 & 40 \\
\hline 16 & 5.3 & 19.4 & 70 & 20.2 & 63.6 & 70 \\
\hline 17 & 5.7 & 31.2 & 80 & 19.8 & 66.8 & 70 \\
\hline 19 & 8.0 & 14.5 & 40 & 35.1 & 91.0 & 60 \\
\hline 20 & 4.9 & 13.5 & 60 & 21.1 & 51.2 & 60 \\
\hline Average & $\mathbf{7 . 1}$ & $\mathbf{2 1 . 5}$ & $\mathbf{6 0}$ & $\mathbf{3 5 . 4}$ & $\mathbf{7 8 . 0}$ & $\mathbf{6 0}$ \\
\hline
\end{tabular}

Figures 4.15 and 4.16 display 30-minute average and 30-minute standard deviation graphs. Night curves illustrate increasing trends during the session while morning curves show almost constant trends during experiment. From this evidence alone, it could be concluded that sleep deprivation affected the steering performance. Moreover, according to the PERCLOS graphs (Figure 4.2) and video data, throughout those periods when the steering angle power mean and standard deviation showed an increasing trend, the drivers 


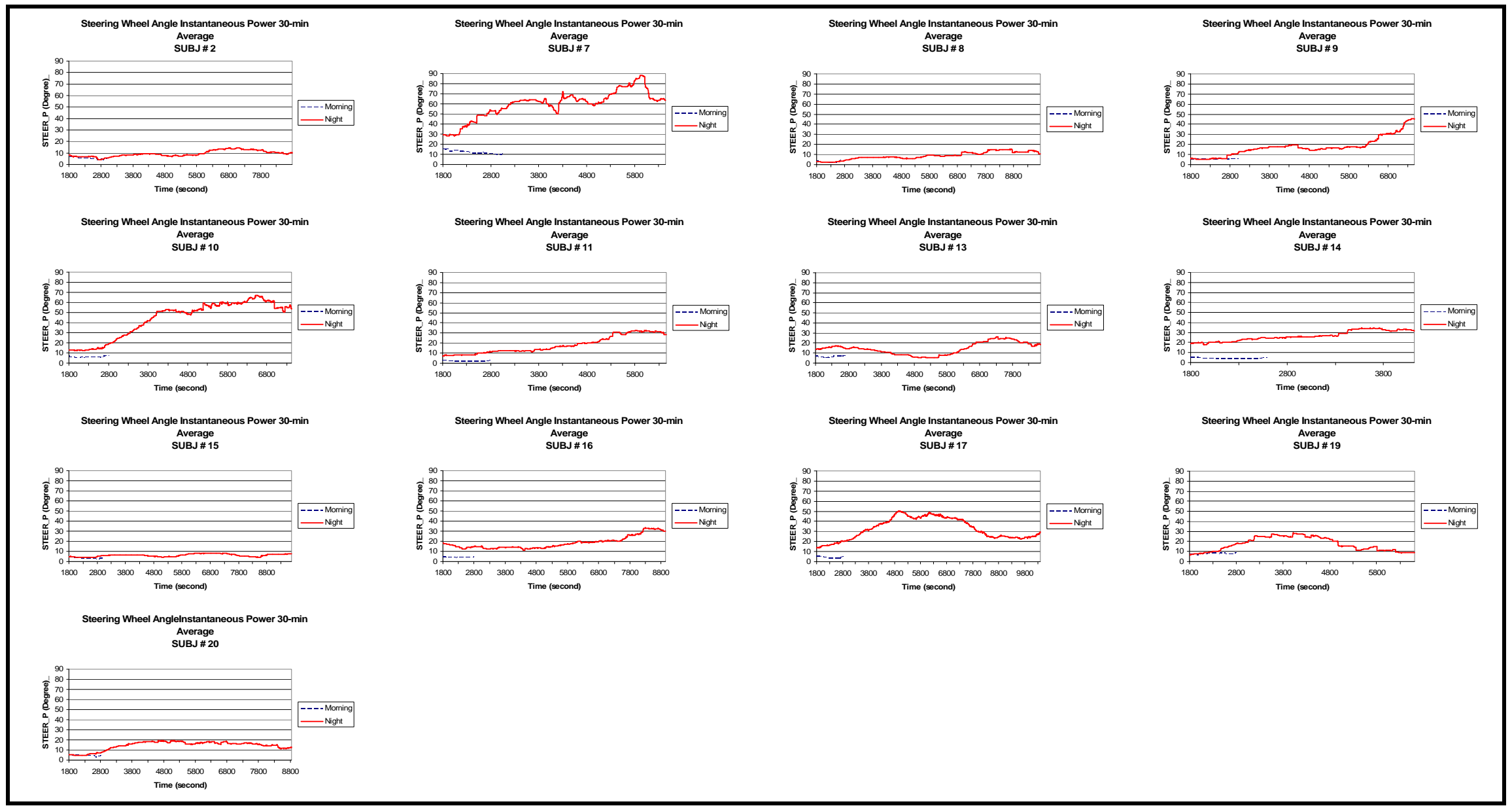

Figure 4.15: Steering wheel angle 30-minute average for morning and night sessions 


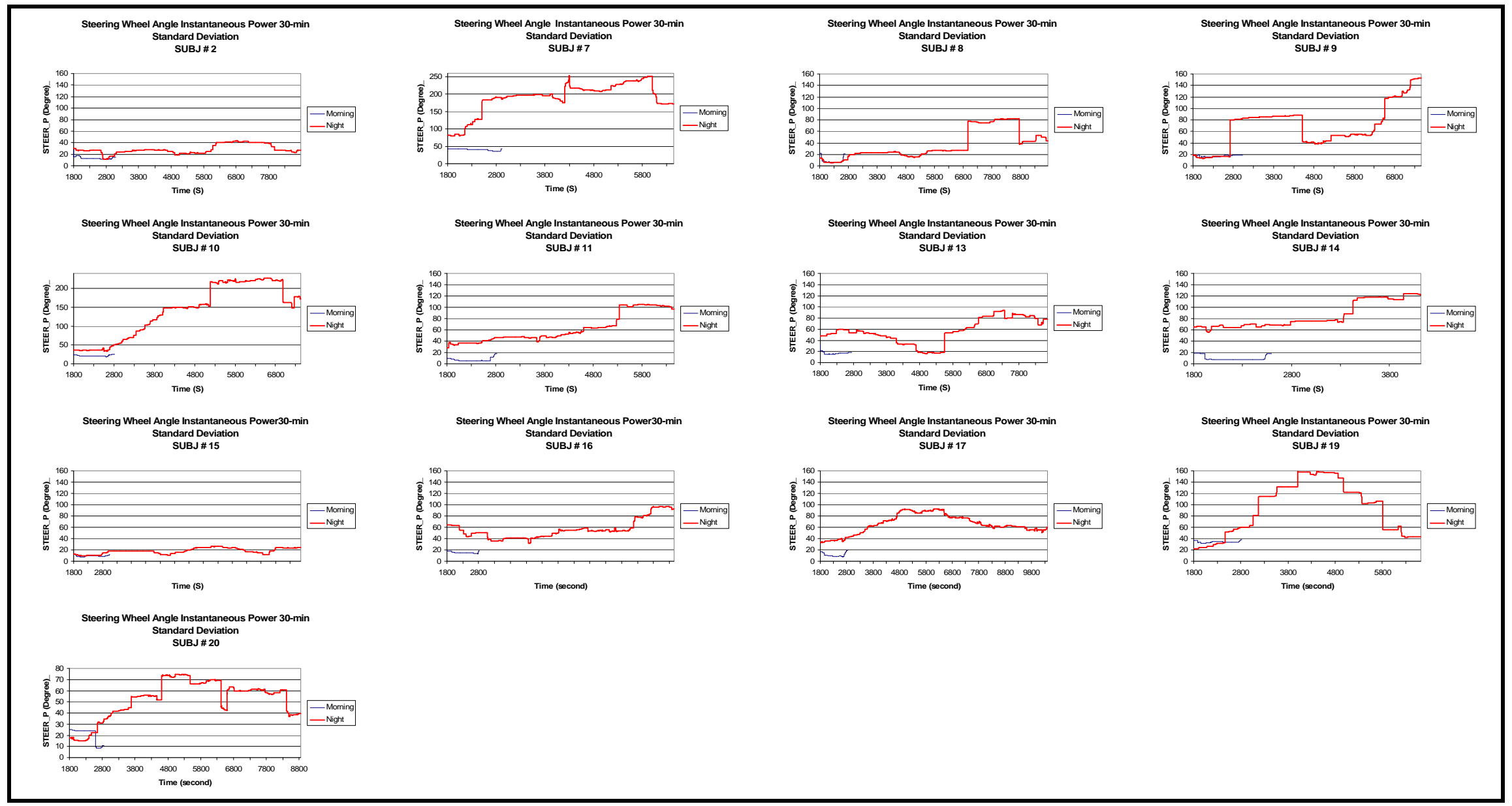

Figure 4.16: Steering wheel angle 30-minute standard deviation for morning and night sessions 
had more prevalence of drowsiness, which confirms the correlation between the steering angle power and drowsiness. Figure 4.17 shows the steering angle 30 min average and standard deviation as well as PERCLOS data for several subjects at the same time. The graphs show a good correlation between the steering and PERCLOS data.

As previously explained, during the impaired phase, the drowsy driver is less sensitive to small corrections and the number of micro-steering wheel adjustments decreases. As a result, the steering angle signal oscillates with large amplitudes and thus has a high power content. Figure 4.18 shows one-minute steering angle power histograms 5 minutes before 12 different crash points, a sample of approximately $10 \%$ of the total crashes. A five-minute time interval before a crash time is chosen in order to guarantee a prevailing driver impaired phase. One-minute steering power histograms randomly chosen from 5 minutes of morning driving data are also plotted in Figure 4.18 to show an example of normal (alert) steering behavior. Since the five-minute intervals selected for both series of data do not correspond to similar driving situations, a direct comparison of both morning and night histograms is not possible. Morning histograms only provide an indication of normal variation of steering angle power, to better assess specific trends in the night data.

In each graph, steering angle power mean, standard deviation, and median are also depicted. In contrast with morning figures, night session histograms show that the steering signals reached higher power values, suggesting the presence of larger steering corrections during the night session. Also, the mean of the steering signal power increases for night driving as compared to morning driving. This indicates that not only were the macro-steering corrections larger during night driving, but the average steering correction was also higher. In addition, during last minute driving before crash (minutes $1-0$ ), only $50 \%$ of the steering data had higher mean and standard deviation values comparing to the other four windows (minutes 5-1), indicating the presence of phase 2 .

The occurrence of high amplitude steering has a random nature; a period of steering macro-movements can be followed by a micro-adjustment interval. In some occasions, the alert drivers' steering signal histograms may contain high power bins because of drivers' inattention that leads to large steering adjustments. Moreover, throughout the night sessions, there were few one-minute window histograms comprising only low power bins, which substantiate the spontaneous nature of impaired phase phenomenon.

On the other hand, during the short dozing off periods, the steering angle signal suddenly flattens with almost constant values. This phenomenon is the result of the described open loop system in which the dozed off driver is not sending any correcting feedback to the steering system. Figure 4.19 highlights this incident for a sample of approximately $10 \%$ of the total number of crashes ( 12 crashes). The dozing-off periods were confirmed by the eye and video data. As shown in the figure, immediately before each crash point, the signal shape is flat. Meanwhile, there were some other steering constant value intervals following a sudden steering move, so called 'drift and jerk'. Drift and jerk phenomenon is the result of a quick recovery from a doze-off to avoid a crash. As described before, on some occasions during the simulation, the driver was fortunate to keep the steering wheel in a correct position keeping the vehicle on the right path during drowsy driving. 


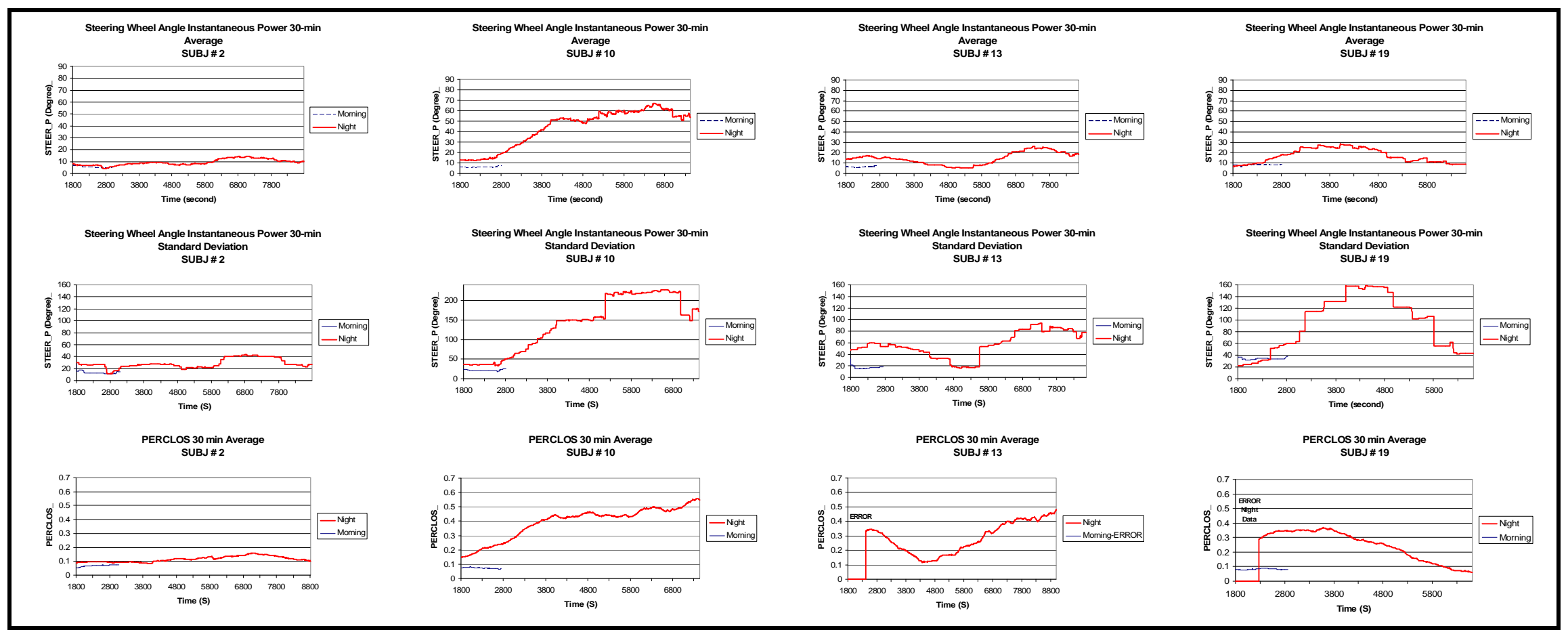

Figure 4.17: Comparison of steering wheel angle graphs and PERCLOS 
SUBJECT \# 7

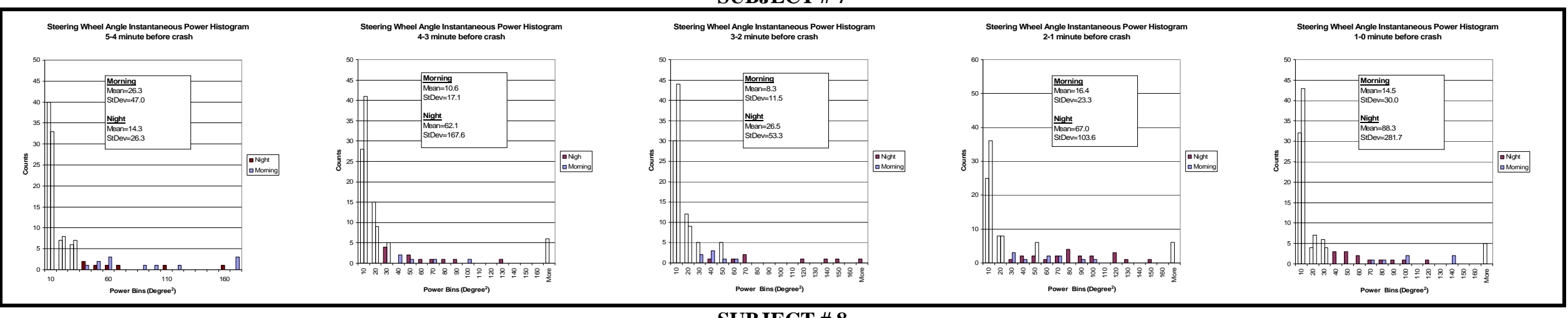

SUBJECT \# 8

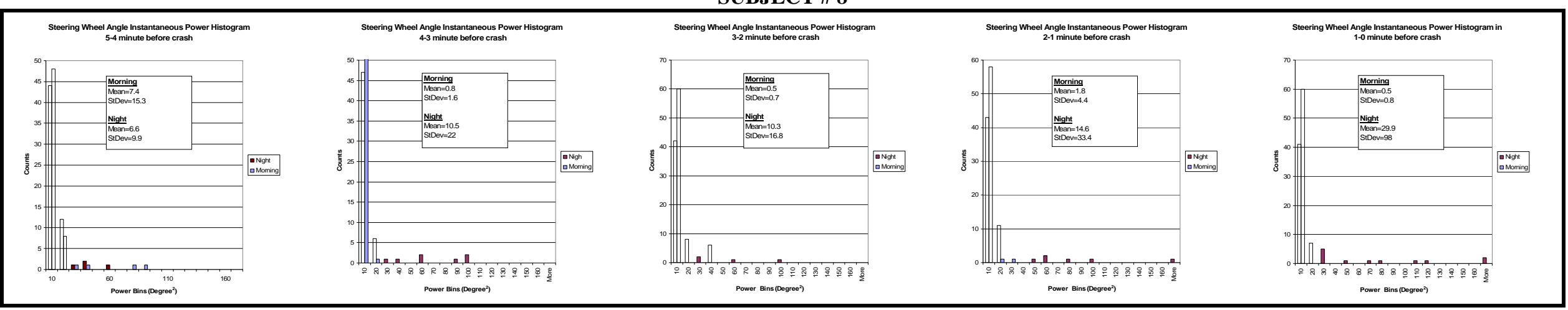

SUBJECT \# 9

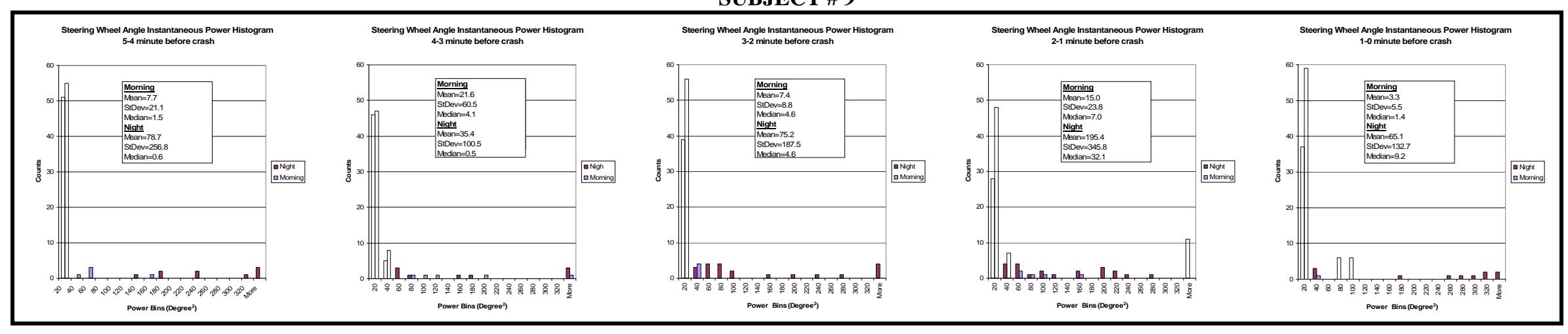

Figure 4.18: Steering wheel angle power 1 to 5 minutes before crash compared to baseline data from morning sessions 
SUBJECT \# 10
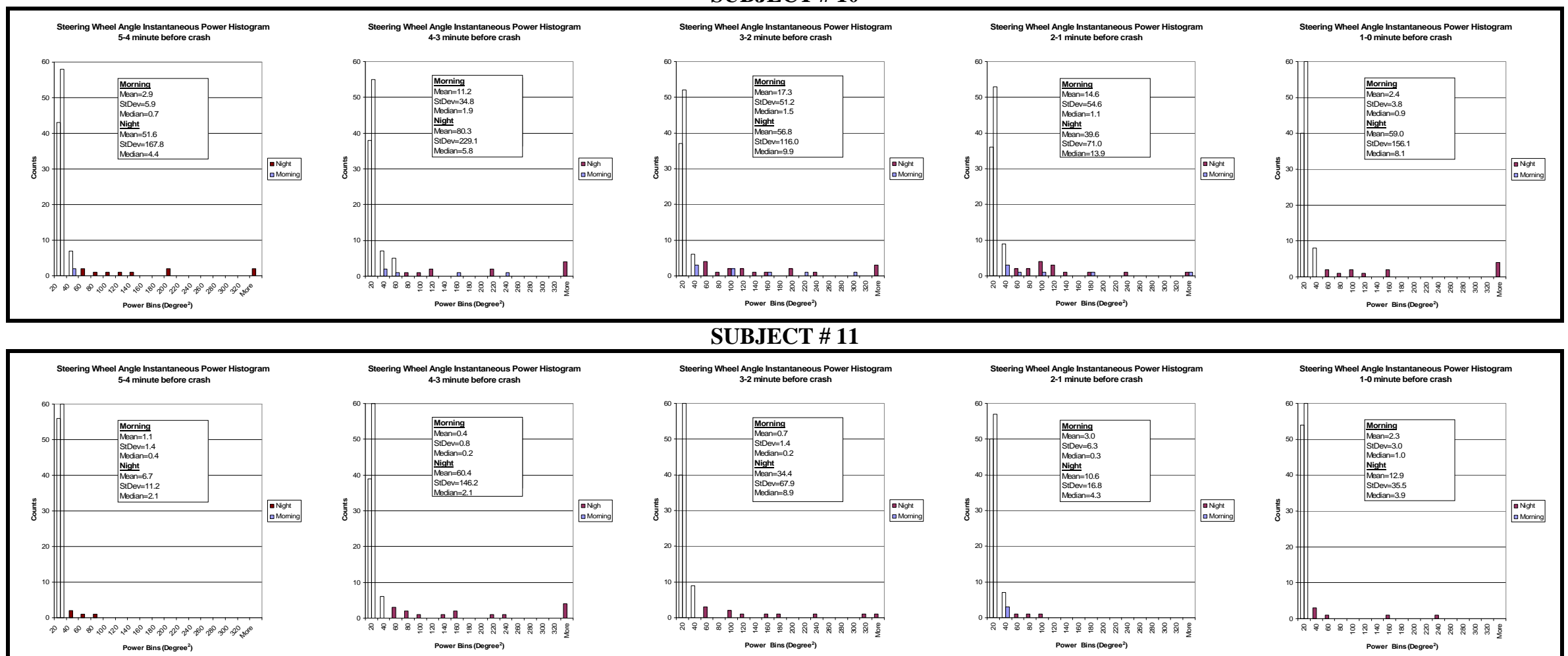

SUBJECT \# 11
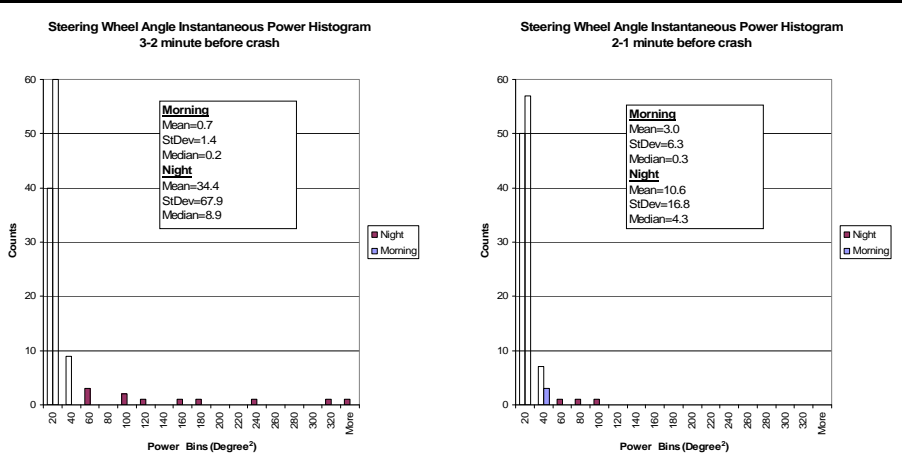

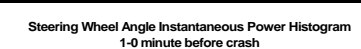

UBJECT \# 11
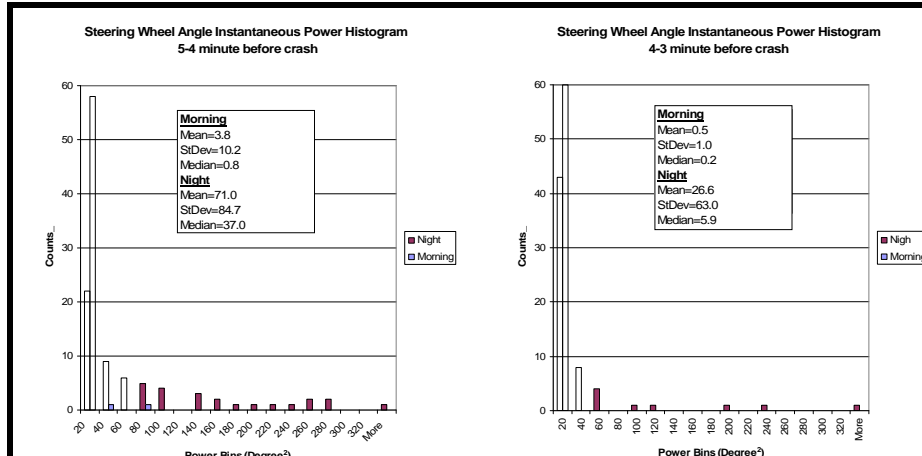

Steering Wheel Angle Instantaneous Power Histogram
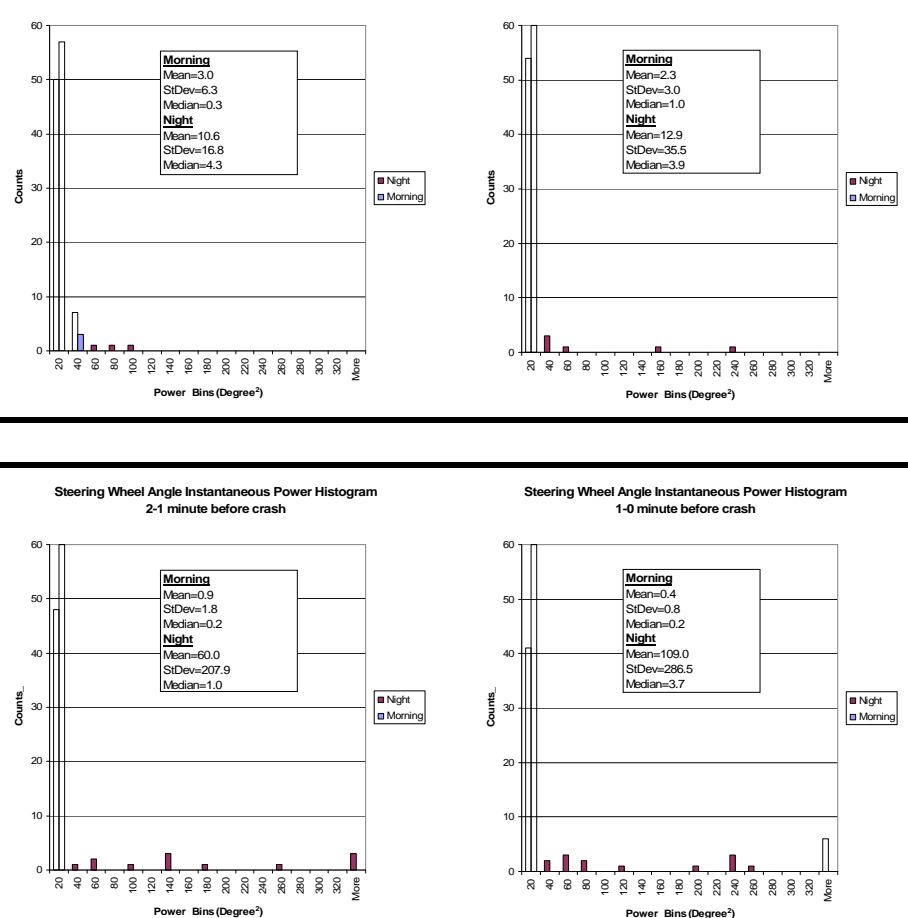

Power ans (Dogree?)

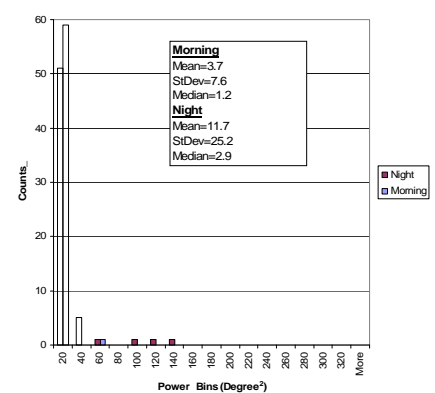

Power Bins (Degree?)

Power Bins (Degree)

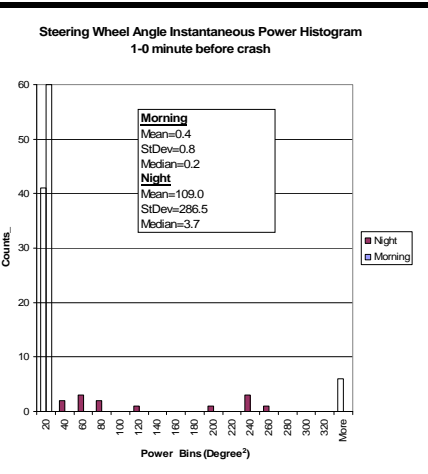

Figure 4.18 (continued) 
SUBJECT \# 13
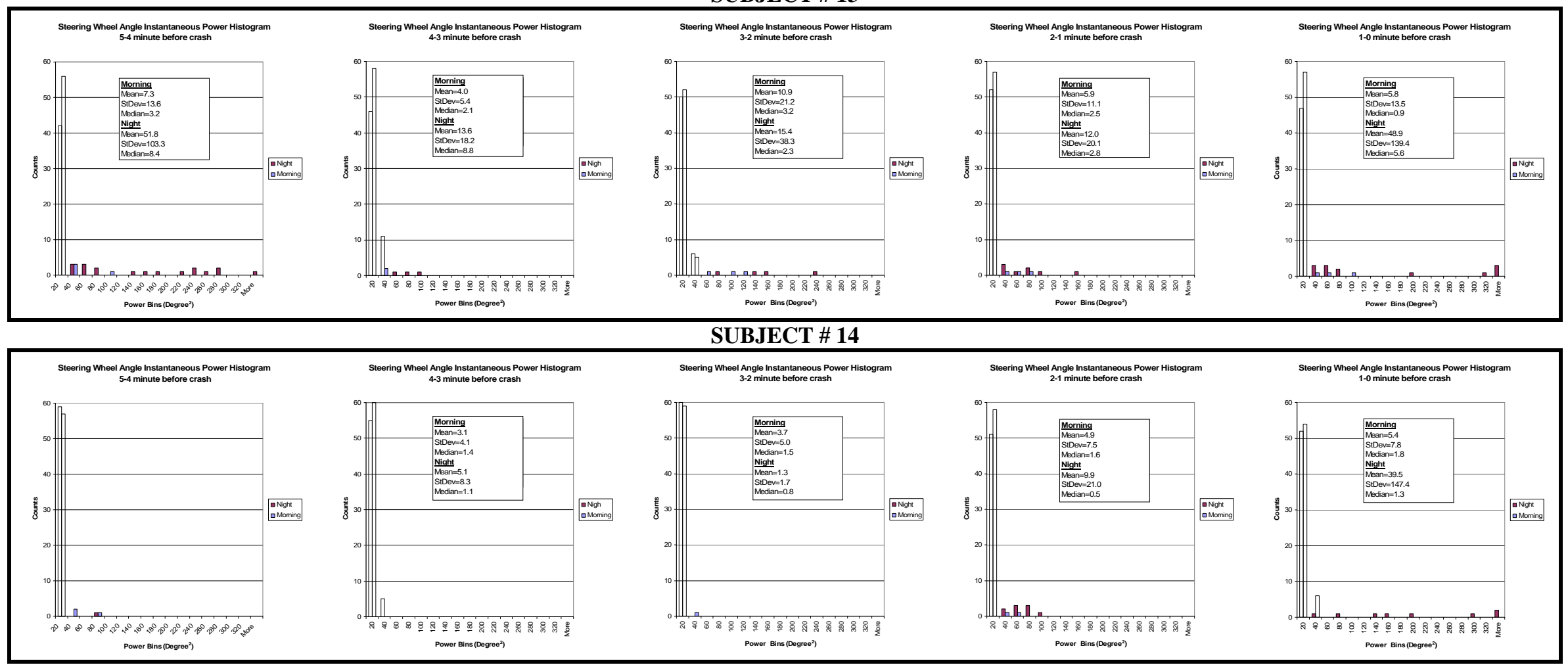

Steering Wheel Angle instantaneous Power Histogram
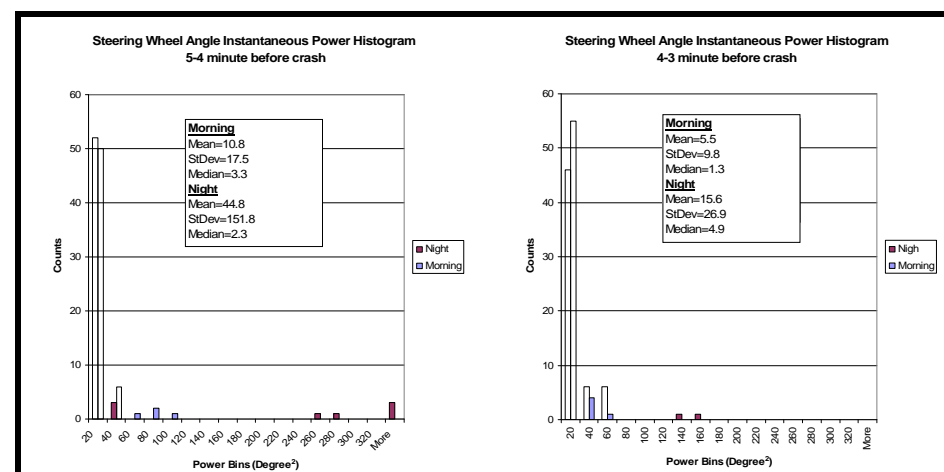

SUBJECT \# 16

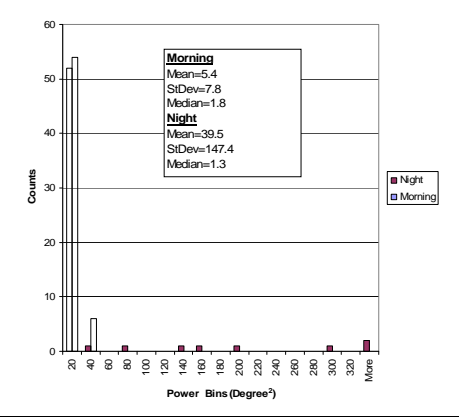

Seering Wheel Angle Instantaneous Power Histogra
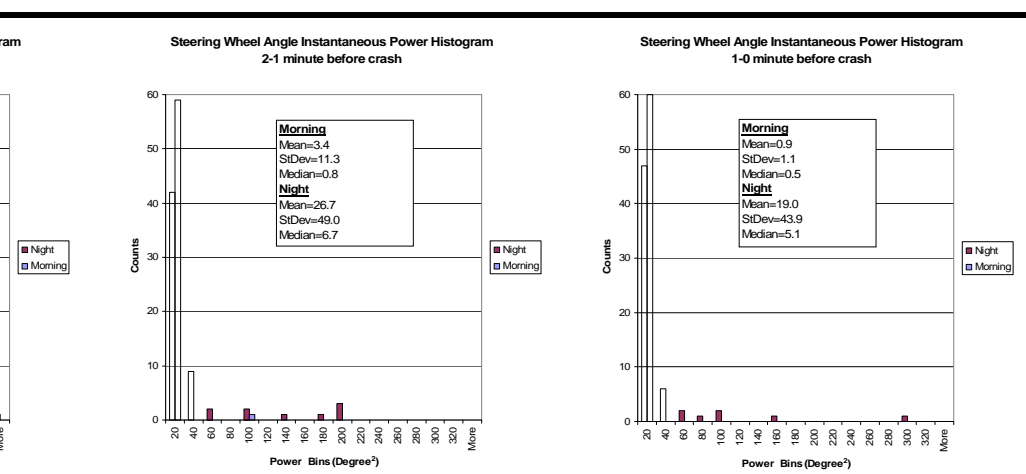

Figure 4.18 (continued) 
SUBJECT \# 17
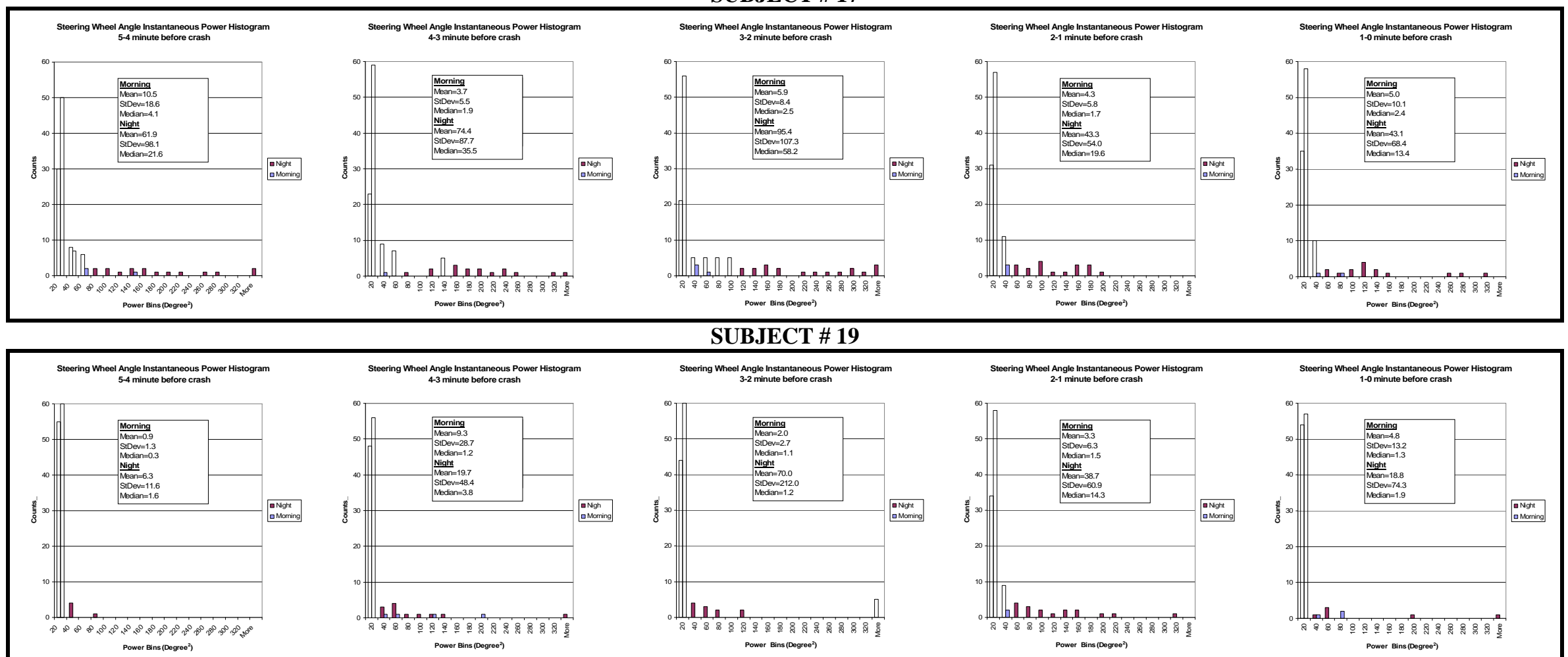

Steering Wheel Angle Intantaneouus Power Histogram
1.- minute before crash

UBJECT \# 20
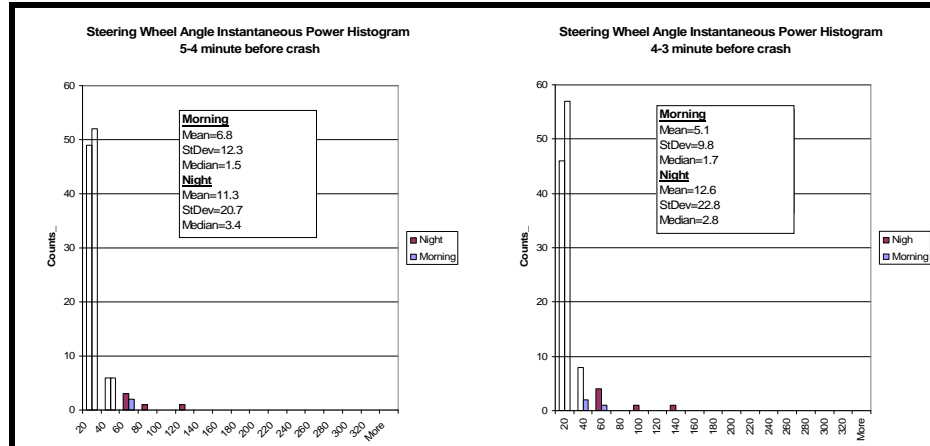

Steering Wheel Angle intantaneous Power Histogram
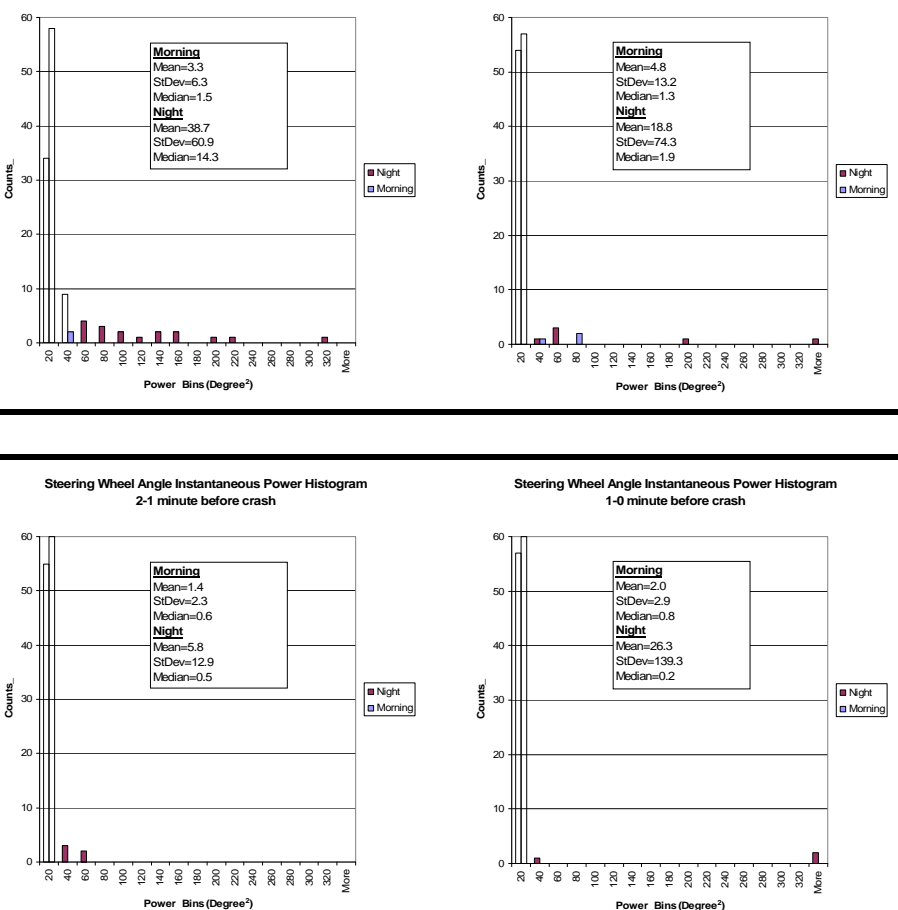

Power Bins (Degree')

Power Bins (Degree)
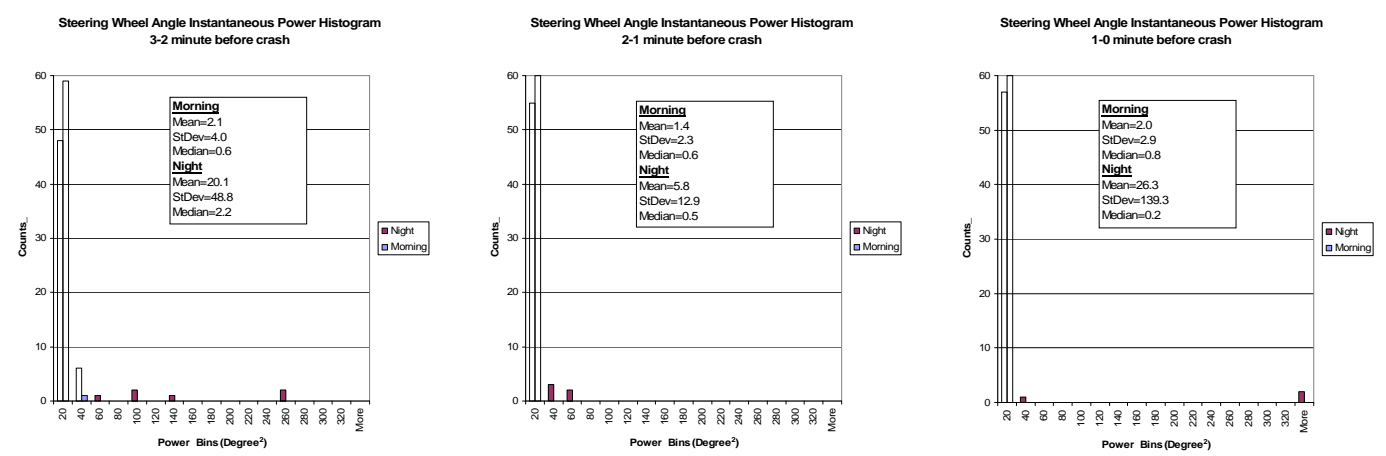

Figure 4.18 (continued) 


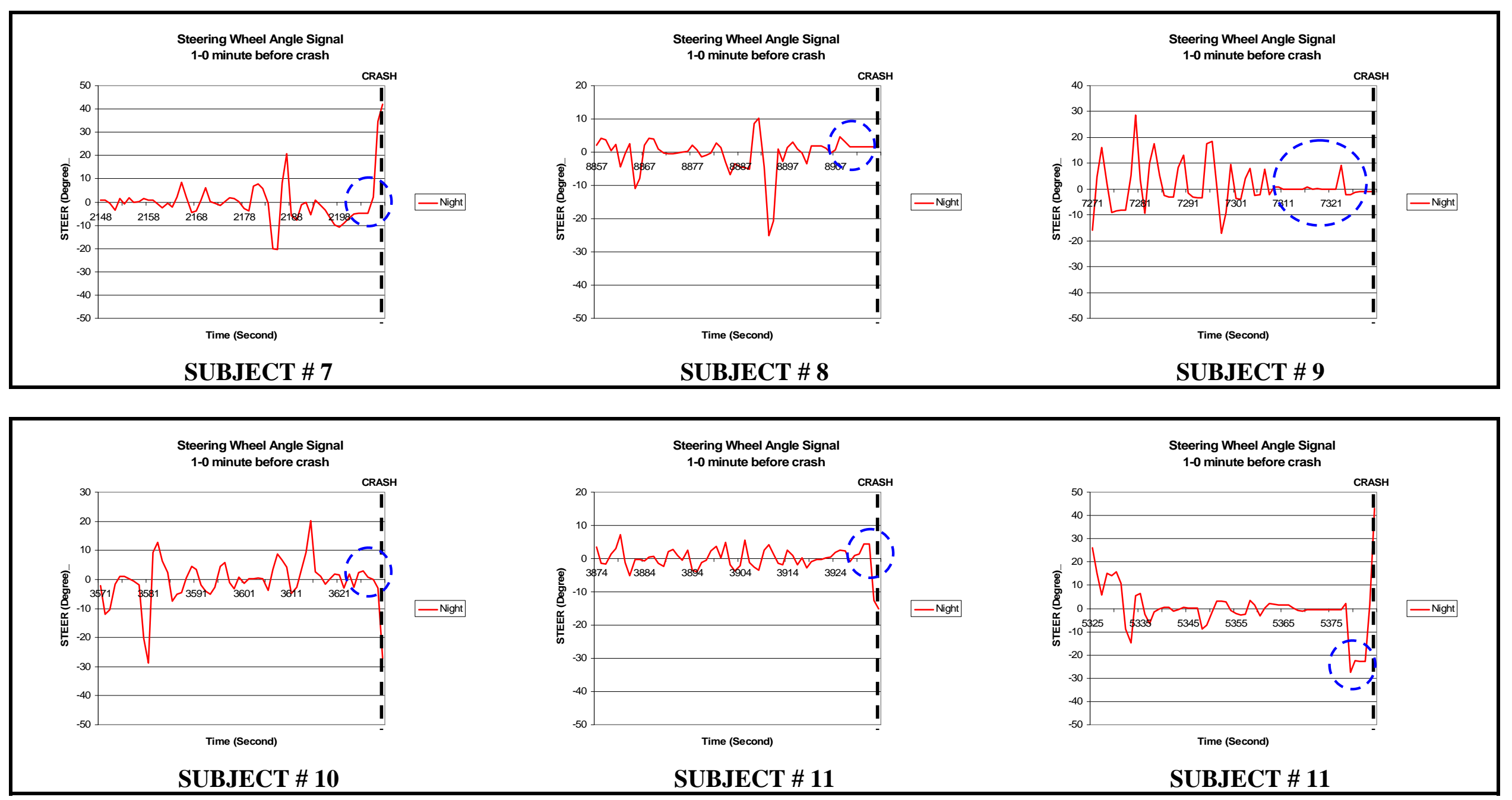

Figure 4.19: Dozing off periods (marked by circles) during one minute before a crash for each subject 

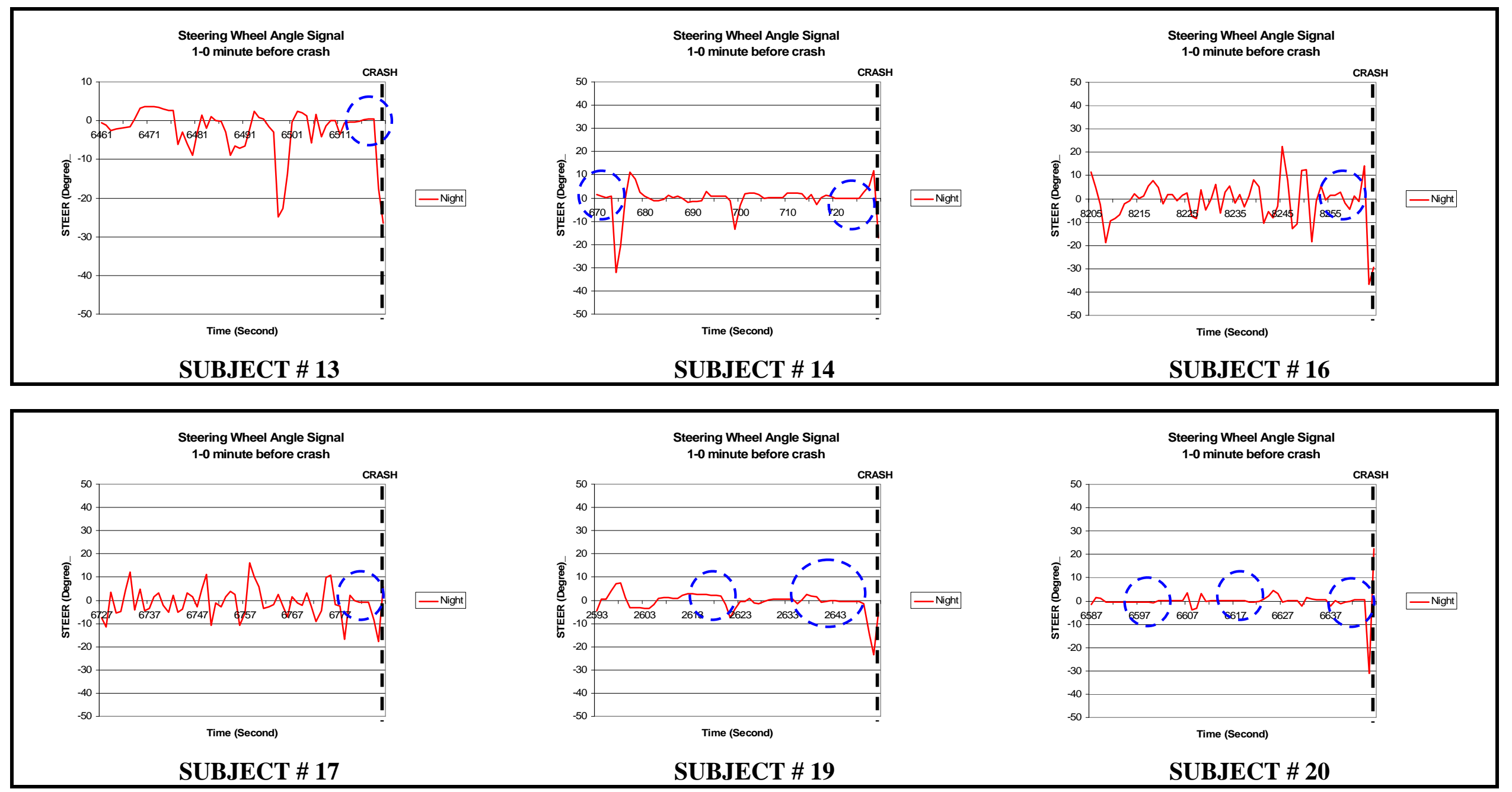

Figure 4.19 (continued) 


\section{Video Recording of Driver Behavior}

Infrared cameras placed inside the vehicle cabin continuously monitored the motion of drivers' hands, body, feet, and face during testing. A total of 26 hours of video stream was recorded during experiment. Comprehensive analysis of this large volume of video data was outside of the scope of this study. However, analysis of the video recordings for two of the tested drivers was performed to give the reader an insight of the available data and possible information that can be drawn from it.

Figure 4.20 shows images of drivers in advanced drowsiness states recorded during experiment.

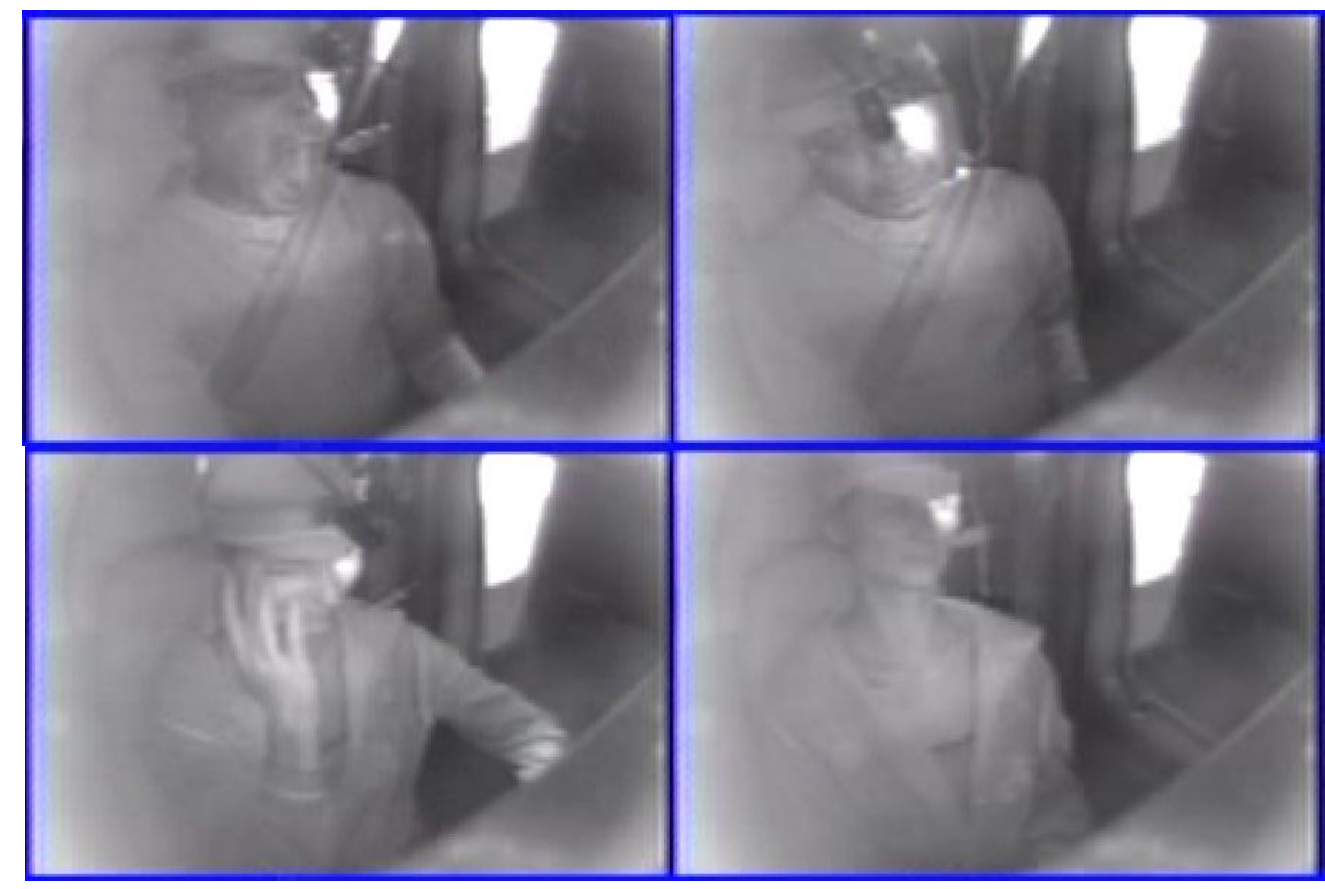

Figure 4.20: Screenshots of drowsy drivers (top left: driver yawning, top right: driver sleeping, bottom left: driver scratching eyes, bottom right: driver with eyes closed)

To analyze the video data, the research team classified drivers' actions in several categories, as listed in Table 4.12. These categories include head and body postures, signs of boredom, mirror checks, driver interaction, and signs of fatigue.

Since drivers drove longer in night sessions than in morning sessions, the number of occurrences of each driver's behavioral variable was normalized by time. Values in Table 4.12 are per hour of driving. Bold numbers are used for night sessions data. Analysis of this data is intricate due to the varying driver behaviors and driving strategies. 
Table 4.12: Drivers' behavioral variables identified through video data (number of occurrences per hour)

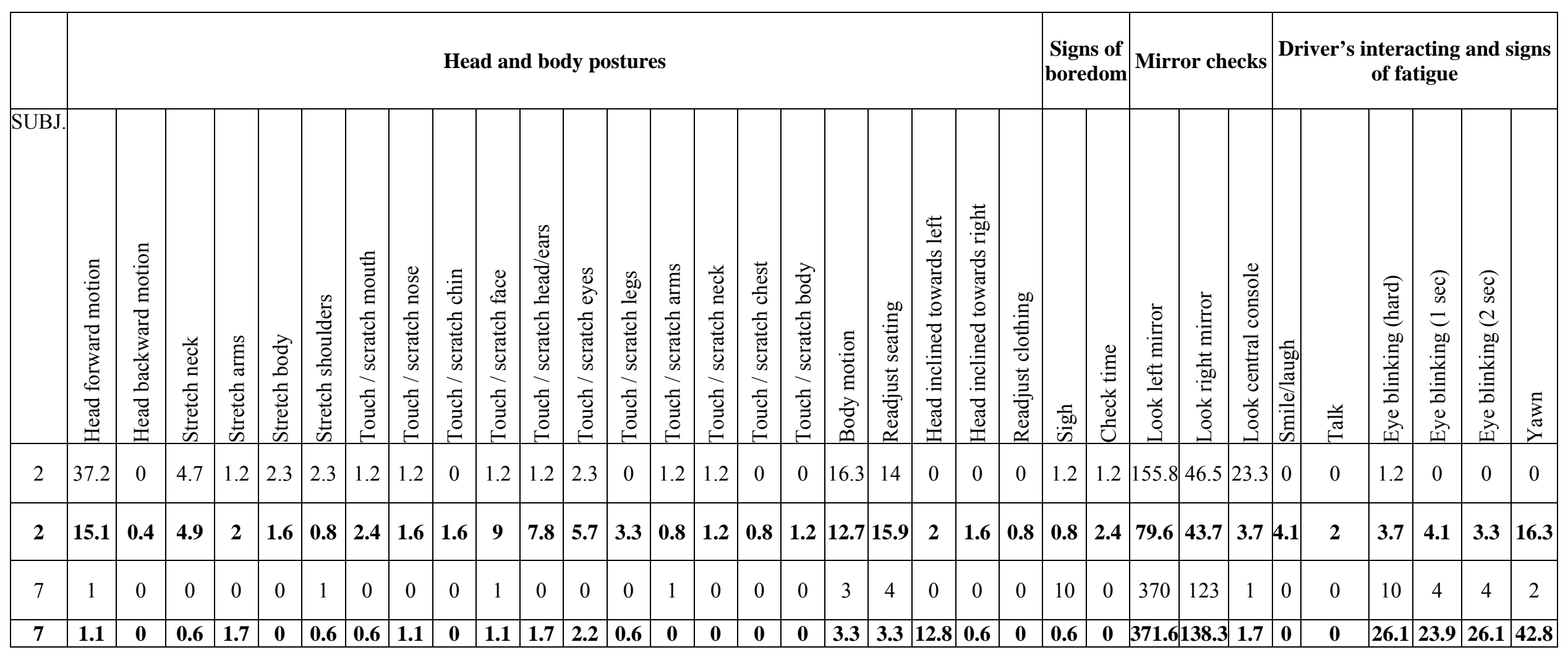




\section{Head and Body Posture}

The head and body posture of a driver can be indicative of drowsiness. Driver No. 2 and Driver No. 7, for example, exhibited a range of this type of behavior.

Driver No. 2 showed a high number of head motions during testing. For example, this driver exhibited a reflexive head nod after checking her side mirrors. Her head motions are significantly less frequent during night session than morning session, implying a possible correlation with drowsiness. The number of times Driver No. 2 touches or scratches her chin, face, head, ears, eyes, and legs significantly increased in the night session comparing to the morning session. These behaviors were especially frequent during drowsy periods and can be interpreted as actions used by Driver No. 2 to fight against fatigue.

Similar observations could be made for Driver No. 7 with smaller differences between night and morning driving. However, Driver No. 7 adopted a different behavior during drowsy periods as he was very often inclined to turn his head to the left to relieve muscular tension from his neck. Seating positions did not change for both drivers between morning and night sessions. Drivers readjusted their seating on a regular basis after some time spent behind the wheel. This behavior does not appear to be related to drowsiness/fatigue but is only a function of time spent driving.

\section{Signs of Boredom}

The video of the drivers can also provide some capability to differentiate between fatigue and monotony. Driver No. 2 checked the time more frequently during night session as compared to morning session, especially at the end of the experiment. Driver No. 7 sighed more during the morning session. Although boredom usually depends on the time spent at task, it is complex to analyze many factors external to the driving simulation that can affect drivers' boredom. Driver No. 7 could, for example, have shown signs of impatience during the morning session because he knew he had to go to his regular work day after testing, while for the night session he was not preoccupied by his work schedule after the experiment.

\section{Mirror Checks}

Observations of drivers' behaviors inside the truck cabin confirmed the importance of rear vision for truck driving simulation, as drivers spontaneously checked their side mirrors on a very regular basis when driving. Mirror checking activity decreased during night driving for Driver No. 2 possibly indicating that drowsiness can affect drivers' mirror checking activity. No change could be observed between morning and night sessions for Driver No. 7.

\section{Driver Interaction and Signs of Fatigue}

For both drivers, the videos confirm that eye blinking activity radically increases during drowsy periods. More eye blinks and longer eye blinking times could be observed during the night sessions comparing to the morning sessions. Also, episodes of yawning were more frequent during night sessions, especially for a few minutes before drowsy periods. 
Yawning, if it could be easily detected, would probably be a good input for a drowsiness detection system.

The drivers' hands position on the steering wheel also revealed interesting patterns. Depending on their fatigue level, drivers seemed to place their hands differently on the steering wheel. It could, for example, be observed that alert drivers would mostly drive with their hands placed in the right "10:2" position, as shown at the top of Figure 4.20. As shown in the bottom of the figure, drowsy drivers, on the other hand, tend to adopt a more relaxed position. For example, when drowsy, these subjects drove with both hands at the bottom of the steering wheel, with the right hand steering and the left arm on the side window, or leaning on the steering wheel and driving with the forearms.

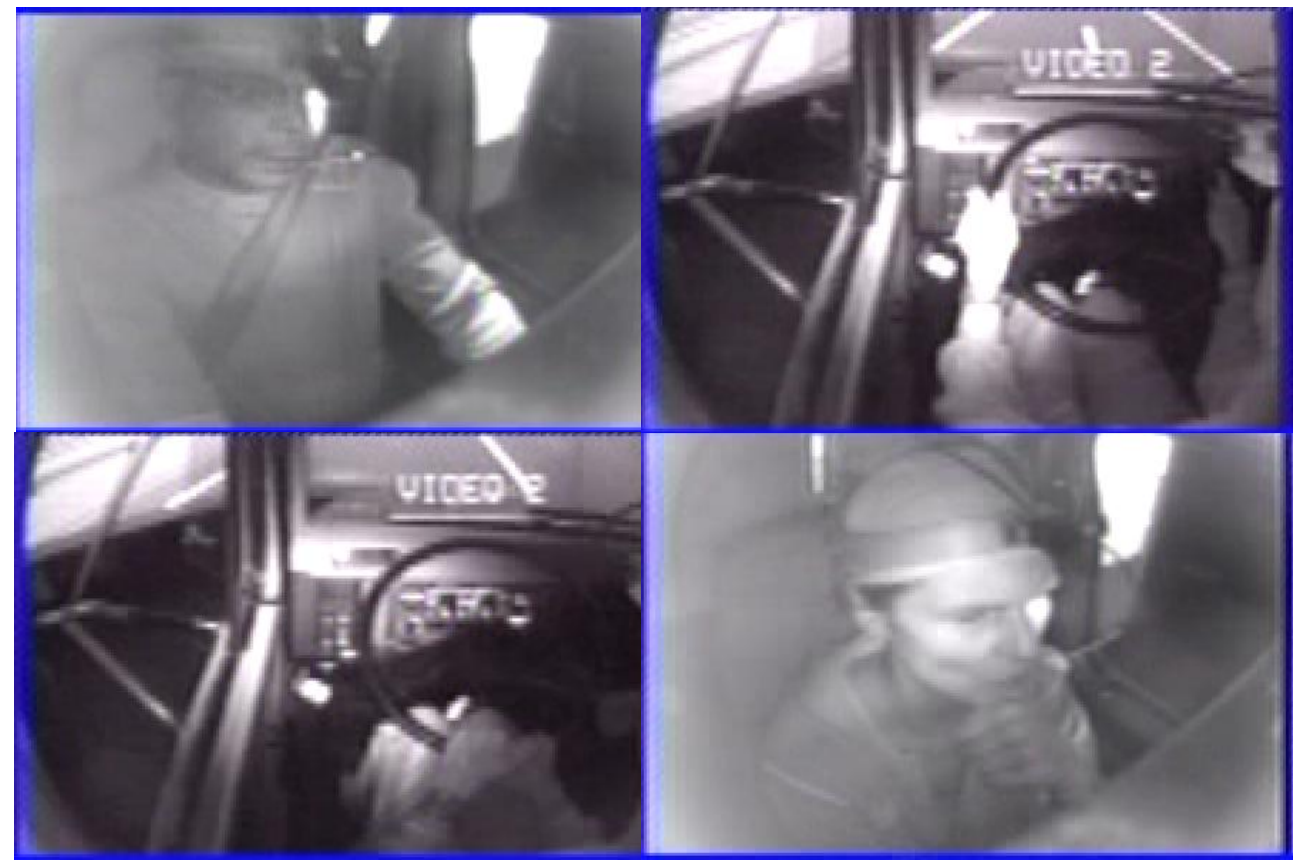

\section{Figure 4.20: Examples of drivers' hands positions on the steering wheel depending on their fatigue level}

Although this video data was primarily collected in this study to help the research team identify drowsy steering data to be used in the Artificial Neural Network detailed in the next chapter of this report, the authors believe that these video recordings contain additional and valuable information that would require more work to be analyzed.

\section{Data Analysis Summary}

The four variables of measures, PERCLOS, vehicle crash, vehicle lateral displacement, and steering wheel angle, showed some correlation with the driver's drowsiness. These variables or measures could be potentially used for drowsiness detection purposes. As mentioned earlier (see section on Eye Closure Data and PERCLOS in this chapter), PERCLOS is not an ideal candidate since capturing eye image is an intrusive process and the measuring methods may present limitations. On the other hand, unobtrusive measurements of lateral displacement and steering wheel angle position can be good 
candidates to evaluate drowsiness in a detection system. The number of crashes directly relates to the driver's drowsiness; however, this data are not suitable for use in a collision prevention system, but is a useful indicator in this study for the onset of sleep episodes.

Variations in vehicle lateral position are a direct consequence of the variations in steering angle. Therefore, only one of them should be used as a drowsiness measure. Steering angle was selected due to the ease of data acquisition.

Sensing of vehicle lateral position is a very complicated process as compared to sensing of steering angle. Two techniques can be cited as examples. In the first method, two video cameras are mounted on front of the vehicle to capture the image of road marking lines. The image is then digitized and processed by an algorithm to determine the position of the vehicle with respect to the center of the lane. In the second method, a magnetic sensor detects the vehicle position with the help of magnets buried in the roadway. Both methods require expensive hardware while sensing of steering angle only needs a potentiometer in the steering column. Capturing of a road image can be very challenging under various road and weather conditions. In addition, putting magnets in the highways across the nation will be a very expensive and is therefore infeasible. Steering can be very accurately detected irrespective of the outside conditions. Therefore, a system based on vehicle lateral displacement will be very costly and complicated. On the other hand, steering-based systems will require negligible maintenance as compared to systems based on vehicle lateral displacement. 


\section{DEVELOPMENT OF ALGORITHMS FOR DRIVER DROWSINESS DETECTION}

The technical approach for the development of the driver drowsiness detection algorithm is based on applying Artificial Neural Networks (ANN) to learn driver steering performance. Measuring driver performance is very challenging due to the complexity, variability, and highly non-linear nature of human behavior. Establishing an exact relationship between a driver's steering activity and driver state of alertness is very difficult as it involves learning human behavior. Adaptive learning techniques, such as ANNs, which can learn from examples and predict the output without knowing the exact relationship between input and output, are ideally suited for modeling human behavior. These techniques present significant advantages over conventional statistical and mathematical methods for capturing the details of human behavior and establishing precise equations relating steering angle activity to drowsiness.

ANNs have been studied and utilized in numerous scientific and engineering fields. One of the main advantages of ANNs is that they infer solutions from data without prior knowledge of the patterns in the data; they extract the patterns empirically, even if the equation between the inputs and the outputs does not exist. This characteristic is very important because in most practical cases, where the exact input-output relationship is very difficult to establish. ANNs also have the ability to generalize (i.e., they respond with a reasonable accuracy to patterns that are broadly similar to the original training patterns). Generalization is very useful because real world data is noisy, distorted, and often incomplete. ANNs are nonlinear, that is, they can solve some complex problems more accurately than linear techniques do. Nonlinear behavior is common but can be very difficult to handle mathematically.

In this study, ANNs were trained to learn the steering behavior of drivers driving under different levels of sleep deprivation in order to identify drowsy driving behavior. The success of the method depends on a unique data-preprocessing scheme, which enables the use of a single ANN for all drivers, by incorporating the variability in human behavior. Two models were developed - one with steering only data and the second one with steering and eye closure data.

Both the steering-with-eye-tracking ANN and the steering-only ANN performed well, at levels analogous to the previous passenger vehicle detection systems. The steering-witheye-tracking ANN had an accuracy of $88 \%$ with a false alarm rate of $9 \%$. The steeringonly ANN had an accuracy of $85 \%$ with a false alarm rate of $14 \%$.

The remainder of this chapter provides a detailed description and evaluation of the ANNs used for the drowsy driver detection system. Specifically, this chapter provides background on artificial neural networks; the data preprocessing scheme used for these ANNs; the neural network architectures; details of their training, validation, and testing; and an evaluation of the drowsy driver detection system in the truck simulator experiment. 


\section{ARTIFICIAL NEURAL NETWORK MODELS}

The architecture of the neural network used in this model is shown in Figure 5.1. The network consists of three layers of neurons.

Input layer: The main function of this layer is input fanning. There is no weight vector associated with the input layer. The number of elements is set equal to the dimension of the input vector $\boldsymbol{X}(\boldsymbol{n})$, as explained in the next section. This layer accepts the input and passes it on to the next layer without any processing.

Hidden layer: This layer is connected to the input layer and output layer by a full synapse. The number of neurons in this layer is selected based on a sensitivity analysis. Input to this layer is the output of the input layer multiplied by the $\mathrm{n}$-dimensional weight vectors of the hidden layer neurons. The output of this layer is an n-dimensional vector.

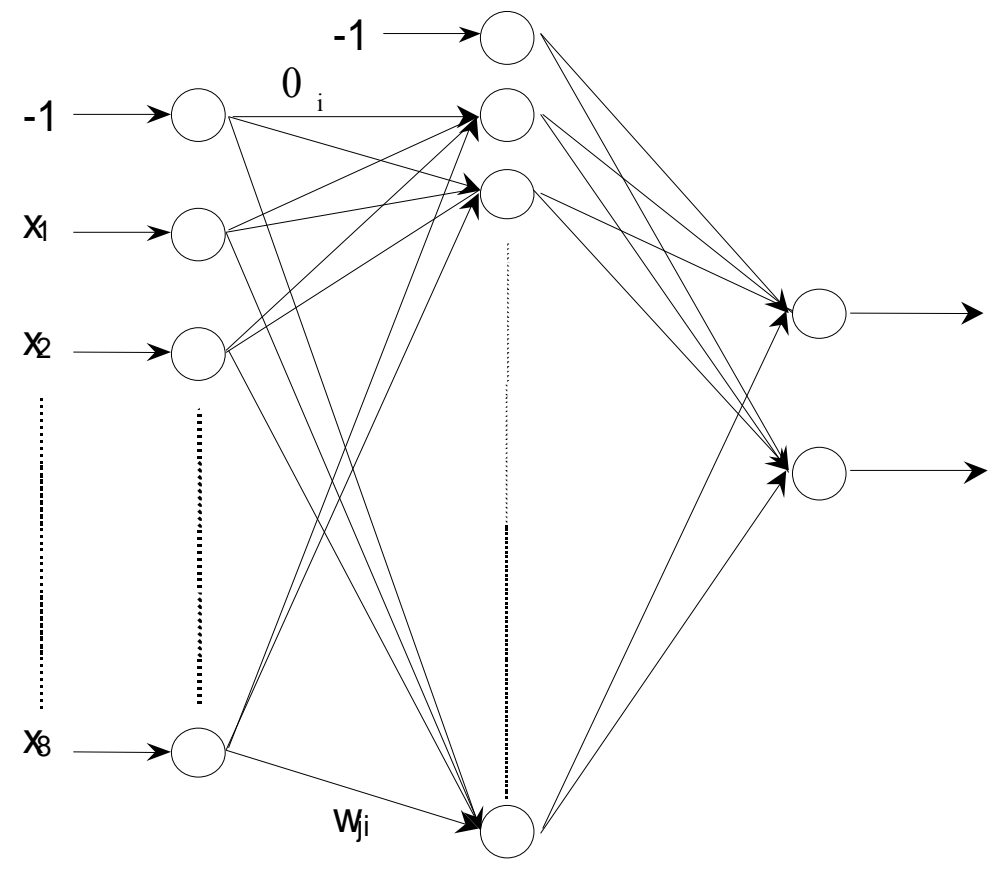

Input Layer Hidden Layer Output Layer

Figure 5.1: Artificial neural network architecture

Output layer: The number of neurons in this layer corresponds to the number of dimensions of the desired output vector. Input to the neurons of this layer is the ndimensional vector (output of hidden layer) multiplied by the n-dimensional weight vector of the output layer neurons. During training, the output layer is provided with the desired output vector. The output of this layer is an n-dimensional vector. 
These layers are connected by full synapse (i.e., each input layer neuron is connected to each neuron of the hidden layer and similarly the hidden layer is fully connected to the output layer). The first neuron with -1 as input in Figure 5.1 represents the threshold or bias.

\section{Training Algorithm (Supervised ANN)}

For training, the network weights and thresholds are updated using an error back propagation-learning algorithm. This algorithm is summarized as follows:

$$
\begin{aligned}
& \mathrm{W}_{\mathrm{ji}}=\text { Synaptic weight connecting neurons } \mathrm{i} \text { and } \mathrm{j} . \\
& \mathrm{v}_{\mathrm{j}}=\text { Internal activity of neuron } \mathrm{j} \text {. } \\
& \mathrm{y}_{\mathrm{j}}=\text { Output of neuron } \mathrm{j} \text {. } \\
& \mathrm{n}=\text { Iteration Number }
\end{aligned}
$$

1. Initialize the synaptic weights and the thresholds to small random numbers.

2. Present the network with an epoch of training exemplars.

3. Apply input vector $X(n)$ to the input layer and the desired response $D(n)$ to the output layer of neurons. The output of each neuron is calculated as follows:

$$
\mathrm{y}_{\mathrm{j}}(\mathrm{n})=\varphi\left(\mathrm{v}_{\mathrm{j}}(\mathrm{n})\right)
$$

$\varphi()$ is the activation function, which maps the neuron's input infinite domain to a prespecified range.

$$
v_{j}(n)=\sum_{i=0}^{p} w_{j i}(n) x_{i}(n)
$$

where

$\mathrm{p}=$ No. of neurons in the layer

$\mathrm{x}_{\mathrm{i}}(\mathrm{n})$ is input to neuron $\mathrm{i}$

For the output layer, the error signal is computed as:

$$
\mathrm{e}_{\mathrm{k}}(\mathrm{n})=\mathrm{d}_{\mathrm{k}}(\mathrm{n})-\mathrm{o}_{\mathrm{k}}(\mathrm{n})
$$

where $o_{k}(n)$ is the output of neuron $i$ in the output layer, and $d_{k}(n)$ is the desired output for neuron $i$ in the output layer. 
4. Adjust the synaptic weights and thresholds of the network by back propagating the error through the network. Weights are adjusted by the amount $\Delta \mathrm{w}$ given by equation (5.4).

$$
\Delta w_{j i}=\frac{1}{N} \sum_{n=1}^{N} \delta_{j}(n) x_{i}(n)
$$

where $\delta_{\mathrm{j}}(\mathrm{n})$ is the local gradient given by:

$$
\begin{aligned}
\delta_{\mathrm{k}}(\mathrm{n}) & =\mathrm{e}_{\mathrm{k}}(\mathrm{n}) \varphi^{\prime}\left(\mathrm{v}_{\mathrm{k}}(\mathrm{n})\right) \quad \text { for the output layer } \\
\delta_{\mathrm{j}}(\mathrm{n}) & =\varphi^{\prime}\left(\mathrm{v}_{\mathrm{j}}(\mathrm{n})\right) \sum_{\mathrm{k}=1}^{\mathrm{m}} \delta_{\mathrm{k}}(\mathrm{n}) \mathrm{w}_{\mathrm{kj}}(\mathrm{n}) \quad \text { for the hidden layer } \\
\mathrm{m} & =\text { No. of neurons in the output layer. } \\
\mathrm{N} & =\text { No. of training sets in one epoch. }
\end{aligned}
$$

The new weights are given by:

$$
w_{j i}(n+1)=w_{j i}(n)+\eta \Delta w_{j i}(n)+\alpha \Delta w_{j i}(n-1)
$$

where $\eta$ is the learning rate parameter. The value of this parameter affects the convergence rate and is determined experimentally, and $\alpha$ is the momentum constant. Typically the value is between 0.1 and 0.9 . This term enables the training process to skip local minima and move toward the global minima.

5. Iterate the computation by presenting new epochs of training examples until the mean square error (MSE) computed over entire epoch achieves a minimum value. MSE is given by:

$$
\operatorname{MSE}=\frac{1}{2 \mathrm{~N}} \sum_{\mathrm{n}=1}^{\mathrm{N}} \sum_{\mathrm{k}=1}^{\mathrm{m}} \mathrm{e}_{\mathrm{k}}^{2}(\mathrm{n})
$$

\section{Training Parameters}

There are many parameters that affect the training performance of an ANN. Some of these parameters are discussed. For more details, please refer to Haykin, S. (1994).

\section{Transfer Function}

The transfer function, also called activation function, maps the neuron's input (possibly) infinite domain to a pre-specified range. Although the number of activation functions can 
be infinite, four famous functions are regularly employed for most engineering applications:

i- Linear Function: The linear function produces a linearly modulated output from the input $x$ as described by the relation:

$$
f(x)=a x
$$

where $x$ ranges over the real numbers, and $a$ is a real scalar.

ii- Step Function: The step function, produces only two values, $b$ and - $c$. If the input $x$ equals or exceeds a predefined value $\theta$, then the function produces the value $b$; otherwise it produces the value $-c$, where $b$ and $c$ are positive scalars. Mathematically this function is described as:

$$
f(x)=\left\{\begin{array}{cc}
b & \text { if } x \geq \theta \\
-c & \text { if } x<\theta
\end{array}\right.
$$

For the assignments $b=1, c=0$, and $\theta=0$, the step function becomes the binary step function. It can be bi-directional binary step function if $c=1$.

iii- Ramp Function : The ramp function is a combination of the linear and step functions. It places upper and lower bounds on the values that the function produces and allows a linear response between the bounds. These saturation points are symmetric around the origin and are discontinuous at the points of saturation. The ramp function is defined as:

$$
f(x)=\left\{\begin{array}{cc}
\gamma & \text { if } x \geq \gamma \\
x & \text { if }|x|<\gamma \\
-\gamma & \text { if } x \leq-\gamma
\end{array}\right.
$$

Where $\gamma$ is the saturation value for the function.

iv- Sigmoid Function: The sigmoid function is a S-shaped continuous version of the ramp function and is a bounded, monotonic, non decreasing function that provides a graded, nonlinear response within a prespecified range. The most common sigmoid function is the logistic function and described mathematically as:

$$
f(x)=\frac{1}{1+e^{-a x}}
$$

Where $a$ is a positive scalar called steepness factor (usually $a=1$ ). The logistic function provides an output value from 0 to 1 . When $a$ becomes $\infty$ in equation (5.12), the slope of the sigmoid function between 0 and 1 becomes infinitely steep and, in effect, it becomes the unipolar step function. An alternative to the logistic sigmoid function is the hyperbolic tangent function:

$$
f(x)=\tanh (a x)
$$


This is a special case of the logistic function. The hyperbolic tangent function shown in Figure 5.2 is symmetric about the origin and preferred for this reason in many engineering applications.

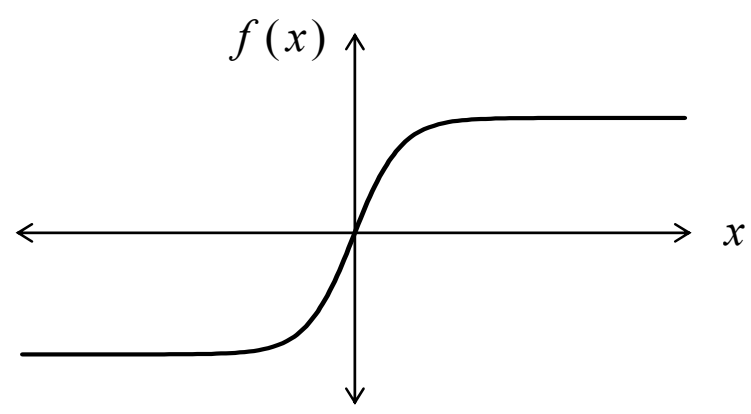

Figure 5.2: Hyperbolic Tangent Function

\section{Effect of Learning Rate}

The effectiveness and convergence of the error back-propagation learning algorithm depend significantly on the value of the learning rate $\eta$. While gradient descent can be an efficient method for obtaining the weight values that minimize the output error, error surfaces frequently possess properties that make the procedure slow to converge.

When broad minima yield small gradient values, then a larger value of $\eta$ will result in a more rapid convergence. However, for problems with steep and narrow minima, a small value of $\eta$ must be chosen to avoid overshooting the solution. This leads to the conclusion that $\eta$ should be chosen experimentally for each problem. Some successful adaptive techniques have been reported in the literature to adaptively change the learning rate during the training process in order to accelerate the convergence and therefore reduce the training time for the BP networks (Haykin, 1994).

\section{Effect of the Momentum Term}

The momentum term allows the network to respond not only to the local gradient, but also to the recent trends in the error surface. This term enables the training process to skip the local minima and move toward the global minimum of the error surface. Typically, $\alpha$ is chosen between 0.1 and 0.9 . The momentum term technique is recommended for problems that converge too slowly or for cases when learning is difficult to achieve.

\section{Cross Validation}

During training, there is always a tendency for the network to memorize the training data and thus fail to generalize beyond this training data. The result of this memorization is that the network performs well only on the data on which it is trained. If it is presented with a data that does not exactly match the training set, the network classification accuracy will be greatly reduced. In order to keep the network from memorizing the 
training data, a separate set of input-output examples is used for cross validation. Every time the weights are updated, MSE calculated from the cross validation data set (which is not a part of the training set) has to be compared with the MSE calculated from the training data set. At any point during training, if the cross validation MSE exceeds the training MSE, training must be stopped because this is an indication of network memorization. A set of data points should be set aside for cross validation during training.

\section{ANN MODEL BASED ON STEERING ANGLE DATA}

This Artificial Neural Network model was trained on data from steering angle only. Data from the steering angle was preprocessed and converted into a vector state representing $\mathrm{n}$ seconds of steering activity before being presented to the model. Training, data preprocessing, and testing phases are discussed in this section. Figure 5.3 shows a schematic of the ANN model based on steering angle data.

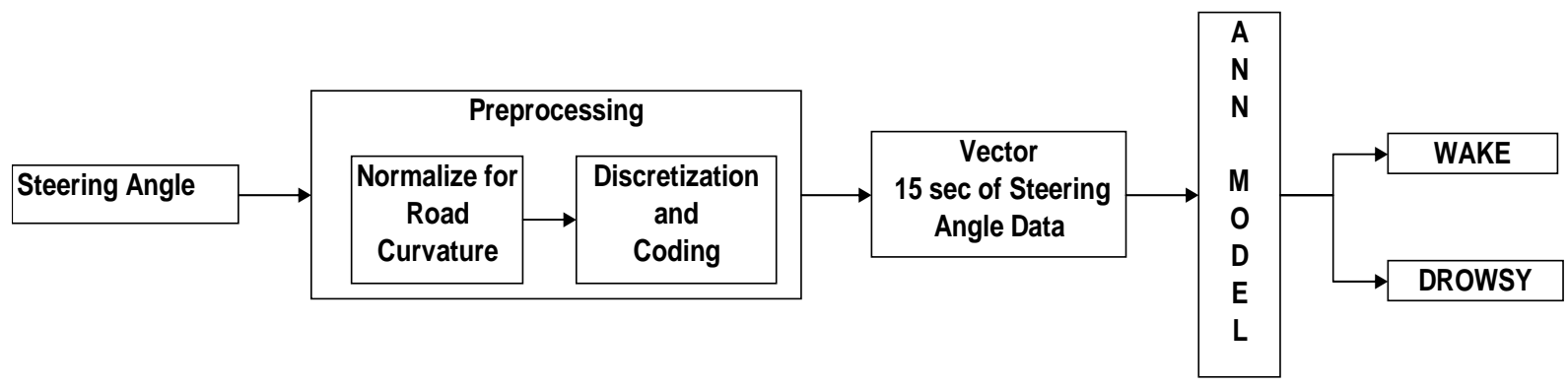

Figure 5.3: ANN model based on steering angle

\section{DATA PREPROCESSING AND INPUT DISCRETIZATION (STEERING ANGLE)}

The successful training of an artificial neural network depends on selecting and preparing proper set of the input data for training. Both steering angle and eye closure data were preprocessed before presenting to the ANN, which allowed a vector state presentation. The data was preprocessed in two steps before presenting to the ANN. In the first step, effect of road curvature was normalized. In the second step, the data was discretized and coded to allow a vector state presentation of the steering wheel angle.

\section{Effect of Road Curvature and Lane Changes}

Road horizontal geometry normally includes two types of geometric sections, straight lines and curves. To simulate the real driving conditions, the test scenario used in the experiment included both straight and curve sections. The steering angle signal recorded in the experiment, therefore, contains two different types of waveforms. In the straight 
sections, the waveforms consist only of the constant steering adjustments to keep the vehicle in the center of the lane, while in the curve sections, the waveforms contain road curvature, in addition to the component of steering adjustments for lane keeping. Data from the curve sections has to be normalized for the effect of curvature. Figure 5.4 shows the two waveforms and the signal after removing the curvature effect.

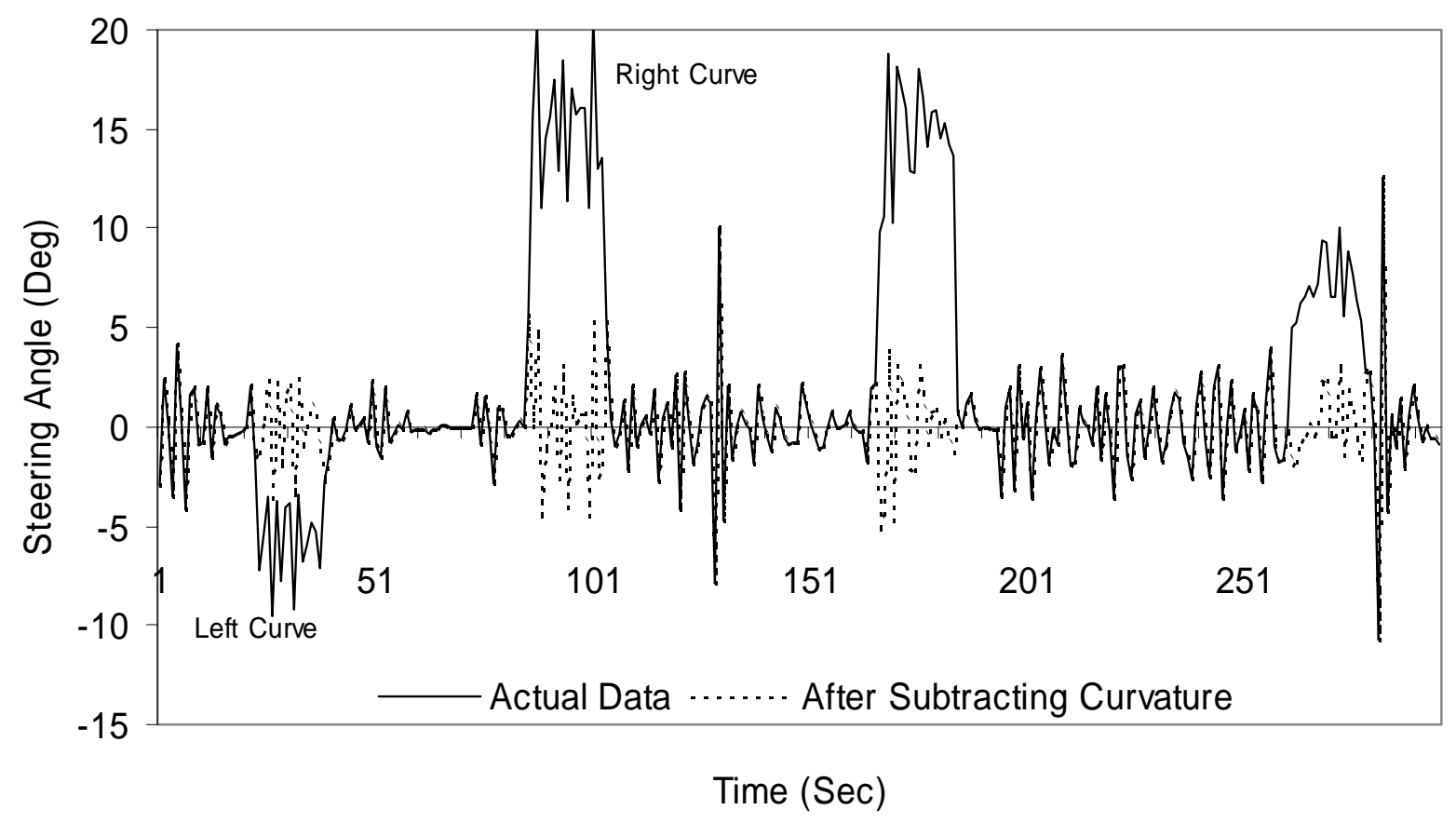

Figure 5.4: Steering angle signal

The effect of curvature was normalized by subtracting the road curvature from the steering angle in the curve sections. This procedure also removed the effect of lane changes from the steering data. The procedure involved is explained as follows:

If four or more consecutive data points are of the same sign (positive for right turn and negative for left turn) and their sum is greater than or equal to 15 (absolute) degrees, then all these points were assumed to be from a curve or portion of a curve section. The mean value of these data points was than subtracted from each of these points. Mathematically, it can be formulated according to the following model: 
Let $\mathrm{s}_{1}, \mathrm{~s}_{2}, \mathrm{~s}_{3}, \mathrm{~s}_{4}, \ldots \ldots \ldots \mathrm{s}_{\mathrm{n}-1}, \mathrm{~s}_{\mathrm{n}}$ be consecutive points of steering wheel angle data

IF $\operatorname{sgn}\left(\mathrm{s}_{1}\right)=\operatorname{sgn}\left(\mathrm{s}_{2}\right)=\operatorname{sgn}\left(\mathrm{s}_{3}\right)=\operatorname{sgn}\left(\mathrm{s}_{4}\right)=\ldots \ldots \ldots \ldots \ldots \operatorname{sgn}\left(\mathrm{s}_{\mathrm{n}-1}\right) \neq \operatorname{sgn}\left(\mathrm{s}_{\mathrm{n}}\right)$

$\operatorname{AND}\left(\mathrm{s}_{1}\right)+\left(\mathrm{s}_{2}\right)+\left(\mathrm{s}_{3}\right)+\left(\mathrm{s}_{4}\right)+\ldots \ldots \ldots \ldots \ldots . . . . . .\left(\mathrm{s}_{\mathrm{n}-1}\right) \geq 15$

THEN, FOR

$\mathrm{i}=1$ to $\mathrm{n}-1$

$$
s_{i}=s_{i}-\frac{\sum_{j=1}^{n-1} s_{j}}{n-1}
$$

Table 5.1 shows an example of this procedure.

Table 5.1: Example of steering angle correction in curve section

\begin{tabular}{|c|c|c|}
\hline Steering angle & Mean & Corrected angle \\
\hline 23.5 & \multirow{14}{*}{23.5} & 0 \\
\hline 23 & & -0.5 \\
\hline 24 & & 0.5 \\
\hline 23 & & -0.5 \\
\hline 22.5 & & -1 \\
\hline 23 & & -0.5 \\
\hline 23.5 & & 0 \\
\hline 23 & & -0.5 \\
\hline 23.5 & & 0 \\
\hline 24 & & 0.5 \\
\hline 23 & & -0.5 \\
\hline 25 & & 1.5 \\
\hline 22.5 & & -1 \\
\hline 24 & & 0.5 \\
\hline
\end{tabular}

More and more of the modern vehicles are equipped with navigation systems with GPS and GIS (Geographic Information System) assistance. The road curvature information can be obtained in advance and proper compensation made when the curve section is actually reached. Also, lane changes can be identified in real-time by the drivers' activation of the turning signals. Steering data could then be compensated for the effect of lane change maneuvers. 


\section{Input Discretization (Steering Angle)}

In step two, the one-dimensional steering angle data was discretized and coded into an eight-dimensional vector.

$$
I(T)=\left[\mathrm{i}_{1}, \mathrm{i}_{\left.2, \ldots \ldots ., \mathrm{i}_{8}\right]}\right]
$$

Discretization is performed according to the following scheme:

Let $\mathrm{r}_{1}, \mathrm{r}_{2}, \ldots \ldots ., \mathrm{r}_{8}$ each represent a discrete range of steering wheel angle. These ranges are defined as follows:

$$
\begin{aligned}
& -\sum_{\mathrm{k}=\mathrm{i}}^{4} \mathrm{p}_{\mathrm{k}}>\mathrm{r}_{\mathrm{i}} \geq-\sum_{\mathrm{k}=\mathrm{i}-1}^{4} \mathrm{p}_{\mathrm{k}} \quad \text { for } \mathrm{i}=1 \rightarrow 4 \\
& \sum_{\mathrm{k}=9-\mathrm{i}}^{4} \mathrm{p}_{\mathrm{k}} \leq \mathrm{r}_{\mathrm{i}}<\sum_{\mathrm{k}=8-\mathrm{i}}^{4} \mathrm{p}_{\mathrm{k}} \quad \text { for } \mathrm{i}=5 \rightarrow 8
\end{aligned}
$$

where $\mathrm{p}_{\mathrm{k}},(\mathrm{k}=0 \ldots 4)$ are constants.

These constants were used to define the discretization ranges. Some drivers are more sensitive to vehicle lateral position and make very accurate corrections to the steering for lane keeping while others are less sensitive and make less accurate corrections. The result is a low amplitude signal for more sensitive drivers and relatively high amplitude signal for less sensitive drivers. Larger values for $\mathrm{p}_{\mathrm{k}}$ will make the discretization ranges wider to accommodate large amplitude while small values will make them shorter for small amplitudes.

With:

p0 $>90$ degree (upper limit on steering angle)

$\mathrm{p} 1, \mathrm{p} 2$, and $\mathrm{p} 3$ are selected according to the driving behavior of individual driver p4 $=0$ (lower limit of steering angle) 
Table 5.2 shows these ranges specifically for $\mathrm{p}_{1}=\mathrm{p}_{2}=\mathrm{p}_{3}=1$. Table 5.3 shows the values of the constants used for discretization.

Table 5.2: Ranges for $\mathbf{p} 1=\mathbf{p} 2=\mathbf{p} 3=1$

\begin{tabular}{|c|c|}
\hline$\underline{\mathbf{r}}_{\mathbf{i}}$ & $\underline{\text { Range }}$ \\
\hline $\mathrm{r}_{1}$ & $-3>\mathrm{r}_{1} \geq-93$ \\
\hline $\mathrm{r}_{2}$ & $-2>\mathrm{r}_{2} \geq-3$ \\
\hline $\mathrm{r}_{3}$ & $-1>\mathrm{r}_{3} \geq-2$ \\
\hline $\mathrm{r}_{4}$ & $0>\mathrm{r}_{4} \geq-1$ \\
\hline $\mathrm{r}_{5}$ & $0 \leq \mathrm{r}_{5}<1$ \\
\hline $\mathrm{r}_{6}$ & $1 \leq \mathrm{r}_{6}<2$ \\
\hline $\mathrm{r}_{7}$ & $2 \leq \mathrm{r}_{7}<3$ \\
\hline $\mathrm{r}_{8}$ & $3 \leq \mathrm{r}_{8}<93$ \\
\hline
\end{tabular}

Table 5.3: Values of constants used for discretization

\begin{tabular}{|c|c|c|c|}
\hline Subject \# & $\mathbf{P}_{\mathbf{1}}$ & $\mathbf{P}_{\mathbf{2}}$ & $\mathbf{P}_{\mathbf{3}}$ \\
\hline 2 & 4 & 4 & 4 \\
\hline 7 & 7 & 7 & 7 \\
\hline 8 & 4 & 4 & 4 \\
\hline 9 & 4.5 & 4.5 & 4.5 \\
\hline 10 & 6 & 6 & 6 \\
\hline 11 & 4 & 4 & 4 \\
\hline 13 & 5 & 5 & 5 \\
\hline 14 & 4 & 4 & 4 \\
\hline 15 & 4 & 4 & 4 \\
\hline 16 & 4 & 4 & 4 \\
\hline 17 & 4.5 & 4.5 & 4.5 \\
\hline 19 & 5 & 5 & 5 \\
\hline 20 & 4.5 & 4.5 & 4.5 \\
\hline 21 & & & \\
\hline & & & \\
\hline 19 & & & \\
\hline 19 & & 4 & 4 \\
\hline
\end{tabular}




\section{Input Coding (Steering Angle)}

After defining the discrete ranges, steering angle was coded into an eight-dimensional vector $\mathrm{I}(\mathrm{T})$. The coding was performed according to the following scheme:

If the amplitude of steering angle falls within the range represented by $r_{i}$ then the $i^{\text {th }}$ component of $\mathrm{I}(\mathrm{T})$ was set equal to 1 and the remaining 7 components were set equal to zero. For example, if the steering angle is 2.5 degrees and $p_{1}=p_{2}=p_{3}=1$, then it will fall in the range represented by $r_{7}\left(2 \leq r_{7}<3\right)$, therefore, only the $7^{\text {th }}$ component of I(T) will be 1 and all other components will be 0 , i.e.

$$
I=\left[\begin{array}{l}
0 \\
0 \\
0 \\
0 \\
0 \\
0 \\
1 \\
0
\end{array}\right]
$$

Table 5.4 shows input discretization and coding for a set of values of steering angle.

Table 5.4: Discretization and coding of steering angle data for $\mathrm{p} 1=\mathrm{p} 2=\mathrm{p} 3=1$

\begin{tabular}{|c|c|c|c|c|c|c|c|c|c|}
\hline \multirow{2}{*}{$\begin{array}{l}\text { Time } \\
\text { T sec }\end{array}$} & \multirow{2}{*}{$\begin{array}{l}\text { Steering } \\
\text { Angle }\end{array}$} & \multicolumn{8}{|c|}{$I(T)=\left[\mathbf{i}_{1}, \mathbf{i}_{2}, \ldots \ldots \ldots \ldots, \mathbf{i}_{8}\right]$} \\
\hline & & $\mathbf{i}_{1}$ & $\mathbf{i}_{2}$ & $\mathbf{i}_{3}$ & $\overline{\mathbf{i}_{4}}$ & $\overline{\mathbf{i}_{5}}$ & $\mathbf{i}_{6}$ & $\mathbf{i}_{7}$ & $\overline{\mathbf{i}_{8}}$ \\
\hline 1 & 1.1 & 0 & 0 & 0 & 0 & 0 & 1 & 0 & 0 \\
\hline 2 & -3.1 & 1 & 0 & 0 & 0 & 0 & 0 & 0 & 0 \\
\hline 3 & -2.2 & 0 & 1 & 0 & 0 & 0 & 0 & 0 & 0 \\
\hline 4 & 0.8 & 0 & 0 & 0 & 0 & 1 & 0 & 0 & 0 \\
\hline 5 & -1.7 & 0 & 0 & 1 & 0 & 0 & 0 & 0 & 0 \\
\hline 6 & 3.0 & 0 & 0 & 0 & 0 & 0 & 0 & 1 & 0 \\
\hline 7 & 1.1 & 0 & 0 & 0 & 0 & 0 & 1 & 0 & 0 \\
\hline 8 & -3.1 & 1 & 0 & 0 & 0 & 0 & 0 & 0 & 0 \\
\hline 9 & -2.2 & 0 & 1 & 0 & 0 & 0 & 0 & 0 & 0 \\
\hline 10 & 0.8 & 0 & 0 & 0 & 0 & 1 & 0 & 0 & 0 \\
\hline 11 & -1.7 & 0 & 0 & 1 & 0 & 0 & 0 & 0 & 0 \\
\hline 12 & 3.0 & 0 & 0 & 0 & 0 & 0 & 0 & 1 & 0 \\
\hline 13 & 0.8 & 0 & 0 & 0 & 0 & 1 & 0 & 0 & 0 \\
\hline 14 & -1.7 & 0 & 0 & 1 & 0 & 0 & 0 & 0 & 0 \\
\hline 15 & 3.0 & 0 & 0 & 0 & 0 & 0 & 0 & 1 & 0 \\
\hline & $X(n)$ & 2 & 2 & 3 & 0 & 3 & 2 & 3 & 0 \\
\hline
\end{tabular}




\section{Input Data (Steering Angle)}

After coding, vector $I(T)$ was summed over $n$ data points to get the input vectors $\mathbf{X}(\mathbf{n})$.

$$
X(n)=I(T)+I(T+1)+I(T+2)+\cdots \cdots \cdots \cdot \cdot I(T+n-1)
$$

Data was collected at $20 \mathrm{~Hz}$ and was averaged over a one second interval. This signal downsampling did not affect the performance of the neural network and allowed the model to achieve similar results with smaller amounts of data. For $n=15, \mathbf{X}(\mathbf{n})$ now represents 15 seconds of steering activity.

Capturing individual human behavior is a complex phenomenon. Selection of proper values for these constants $\left(\mathrm{p}_{1}, \mathrm{p}_{2}\right.$, and $\left.\mathrm{p}_{3}\right)$ is a topic for further research. Further experiments, with a more diverse population of drivers, are required to study the individual behavior. With more knowledge of individual behavior, we may be able to classify the drivers into groups with similar behavior. With limited data and a population, which is not very diverse, it was not possible to classify drivers based on individual performance. The values of these constants were selected based on visual observation of data and by trial and error.

By changing the values of these constants, the sensitivity of the system can be adjusted. Smaller values will make the system more sensitive and may result in increase number of false alarms while large values will make it less sensitive. In a real life situation, one will start with a high sensitive system and gradually adjust the values until one is comfortable with the number of false alarms.

It can be seen from Table 5.3 that the values of $\mathrm{p}_{1}=\mathrm{p}_{2}=\mathrm{p}_{3}$ ranged from 4 to 5 for the majority of the drivers who participated in this study. This input discretization scheme combines $n$ seconds of steering angle changes (i.e., driver performance) and allows a vector state representation of this variable. This vector state also incorporates a measure for sensitivity of the individual driver steering, which enables the system to use the same network (ANN) for different drivers. By adjusting the sensitivity level for different types of drivers, the need for training a separate ANN for each driver was eliminated.

\section{Desired Output}

An artificial neural network requires a set of training examples consisting of an input and a desired output. The driving intervals represented now by $\mathbf{X}(\mathbf{n})$ are classified into two classes, drowsy and awake. Since no subjective evaluation or any other criteria for classification was available, the driver was assumed to be drowsy if:

- He presented signs of advanced fatigue from test observations (see section on analysis of in-vehicle video recordings in previous chapter)

- His eye closure data reflected a blinking activity associated with drowsiness

These are good indicators of the driver performance. These were considered as qualifiers to indicate sleepy (drowsy) state. For the ANN training purpose, the outcome was classified as drowsy when these conditions were met. 
Most of the data from the end of the night sessions was classified as drowsy. Data from morning session was classified as awake, based on the observed data and lack of sleep deprivation for the drivers. The desired output is a two-dimensional vector, $\mathbf{D}(\mathbf{n})$. Each dimension represents each class.

Table 5.5 shows desired output vector. Table 5.6 shows the format of the final input data to the network.

Table 5.5: Desired output vector

\begin{tabular}{|l|c|c|}
\hline & Awake & Drowsy \\
\hline D(n) & {$\left[\begin{array}{ll}1 & 0\end{array}\right]$} & {$\left[\begin{array}{ll}0 & 1\end{array}\right]$} \\
\hline
\end{tabular}

Table 5.6: Example of input data (steering only)

\begin{tabular}{|c|c|c|c|c|c|c|c|c|c|}
\hline \multicolumn{9}{|c|}{$\mathbf{x}(\mathbf{n})$} & \multicolumn{3}{c|}{ D(n) } \\
\hline $\mathbf{x}_{\mathbf{1}}$ & $\mathbf{x}_{\mathbf{2}}$ & $\mathbf{x}_{\mathbf{3}}$ & $\mathbf{x}_{\mathbf{4}}$ & $\mathbf{x}_{\mathbf{5}}$ & $\mathbf{x}_{\mathbf{6}}$ & $\mathbf{x}_{\mathbf{7}}$ & $\mathbf{x}_{\mathbf{8}}$ & Sleep & Awake \\
\hline 2 & 2 & 3 & 0 & 3 & 2 & 3 & 0 & $\mathbf{0}$ & $\mathbf{1}$ \\
\hline 0 & 1 & 0 & 14 & 0 & 0 & 0 & 0 & $\mathbf{1}$ & $\mathbf{0}$ \\
\hline 2 & 0 & 5 & 4 & 3 & 1 & 0 & 0 & $\mathbf{1}$ & $\mathbf{0}$ \\
\hline 0 & 0 & 2 & 3 & 9 & 1 & 0 & 0 & $\mathbf{0}$ & $\mathbf{1}$ \\
\hline 0 & 0 & 0 & 10 & 5 & 0 & 0 & 0 & $\mathbf{0}$ & $\mathbf{1}$ \\
\hline 0 & 5 & 3 & 6 & 1 & 0 & 0 & 0 & $\mathbf{1}$ & $\mathbf{0}$ \\
\hline 1 & 4 & 1 & 3 & 4 & 0 & 1 & 1 & $\mathbf{1}$ & $\mathbf{0}$ \\
\hline 1 & 5 & 2 & 0 & 5 & 1 & 1 & 0 & $\mathbf{1}$ & $\mathbf{0}$ \\
\hline
\end{tabular}

\section{ANN ARCHITECTURE (STEERING ANGLE)}

The network architecture for this model was similar to the three layer, feed forward network shown in Figure 5.1. Table 5.7 shows a summary of the network configuration.

Table 5.7: Summary of ANN architecture

\begin{tabular}{|c|c|c|c|}
\hline \multicolumn{3}{|c|}{ No. of Neurons } & \multirow{2}{*}{ No of Weights } \\
\cline { 1 - 2 } Input Layer & Hidden layer & Output layer & \\
\hline 8 & 21 & 2 & 210 \\
\hline
\end{tabular}




\section{TRAINING DATA SET (STEERING ANGLE)}

For training to be successful, equal proportions of examples from both classes (awake and drowsy) should be used. Data from 3 minutes after the start of the experiment and 3 minutes before end of the experiment was discarded. The minimum data available from the morning session for every subject was approximately 30 minutes. Since all data from the morning session was classified as awake, only 30 minutes of data from selected portions of night driving was used for training. This corresponds to 100 exemplars from morning and 100 from the night. The number of input data required for training is roughly three times the number of weights in the network. The same number is required for testing network performance during training and about one third of this amount is required for cross validation. Data from 7 subjects (1,400 exemplars) was combined into one file, randomized, and then divided into three files. Table 5.8 shows the number of exemplars in each file.

Table 5.8: Number of exemplars in each data set

\begin{tabular}{|c|c|c|c|}
\hline Number of Weights & Training File & Cross Validation & Testing File \\
\hline 210 & 600 & 200 & 600 \\
\hline
\end{tabular}

It is important to note that the purpose of the training file at this point is to test its performance during the iterative training process. The network is tested further using data from all subjects.

\section{ANN TRAINING (STEERING ANGLE)}

Training an ANN requires selecting the right architecture and optimum (close to optimum, best values) for the various training parameters. The ANN training was performed multiple times with varying parameters until the best results were obtained. For more detail analysis and understanding of ANNs, please refer to Haykin, S. (1994). Table 5.9 summarizes the training performance of the best or optimal ANN architecture.

Table 5.9: ANN performance during training

\begin{tabular}{|c|c|c|}
\hline \multirow{2}{*}{ Best Networks } & \multicolumn{2}{|c|}{ Steering Only Model } \\
\cline { 2 - 3 } & Training & Cross Validation \\
\hline Epoch \# & 100 & 100 \\
\hline Minimum MSE & 0.0638 & 0.0559 \\
\hline Final MSE & 0.0638 & 0.0559 \\
\hline
\end{tabular}


The Steering Angle ANN had the following training characteristics:

- The network was trained in multiple passes. Each pass lasted for a total of 1,000 epochs or when the cross validation error exceeded the training error.

- The network weights and thresholds were updated in batch mode (i.e., weights were updated after presentation of one complete epoch of data).

- Input vector was normalized within $(0,1)$ range before presenting to the network.

- The tan hyperbolic activation function, with output range from -1 to 1 , was applied to neurons of the hidden and the output layers. The hyperbolic tangent function shown in Figure 5.2 is symmetric about the origin and preferred for this reason in many engineering applications.

- Values for the learning rate parameter $(\eta)$ were kept variable through out the training process. Starting with a large value and gradually decreasing to a small value.

- Momentum factor $(\alpha)$ was also kept variable during training.

Figure 5.5 shows Mean Square Error (MSE) for training and cross validation of the network. These graphs indicate the network performance during training. The cross validation error always stayed below the training error showing the network generalization. The smooth drop of MSE indicates that the ANN performed very well.

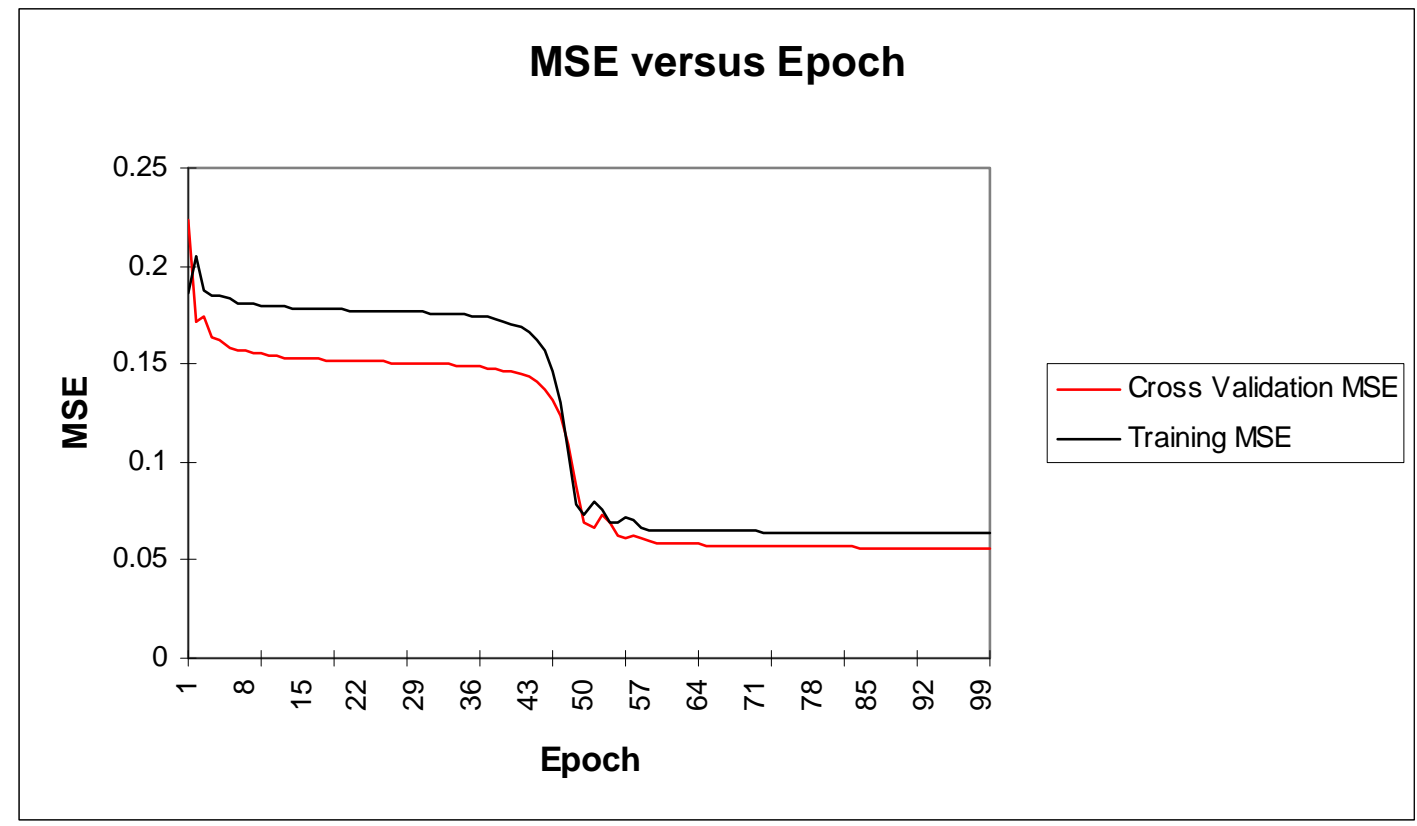

Figure 5.5: ANN training performance 


\section{ANN TESTING (STEERING ANGLE)}

During the training process, the ANN was tested after each iteration to evaluate its performance. Table 5.10 shows network testing performance for the best network weights. The test data set (test file) contained 600 input vectors (each vector representing 15 seconds of driving). The test data was not used in training or cross validation; in other words, the network had never seen this data before.

Table 5.10: Test results

\begin{tabular}{|l|c|c|}
\hline Output/Desired & AWAKE & DROWSY \\
\hline WAKE & 288 & 40 \\
\hline DROWSY & 37 & 235 \\
\hline \% CORRECT & 89 & 85 \\
\hline
\end{tabular}

The network correctly identified 235 out of a total of 275 drowsy intervals (i.e., an accuracy of $85 \%$ ). There were 37 "false alarms", intervals that were in the awake class but were misclassified as drowsy. Also, 40 intervals that were in the drowsy class were misclassified by the network as awake.

\section{ANN MODEL BASED ON STEERING AND EYE DATA}

This model is based on combined data from steering angle and eye closures. The purpose is to see the correlation between the two data sets and to see if the steering model can be further improved by adding eye closure data to the model. Steering data was preprocessed the same way as that of the steering angle model while eye data was processed as explained in the following section. Figure 5.6 shows the schematic of this model.

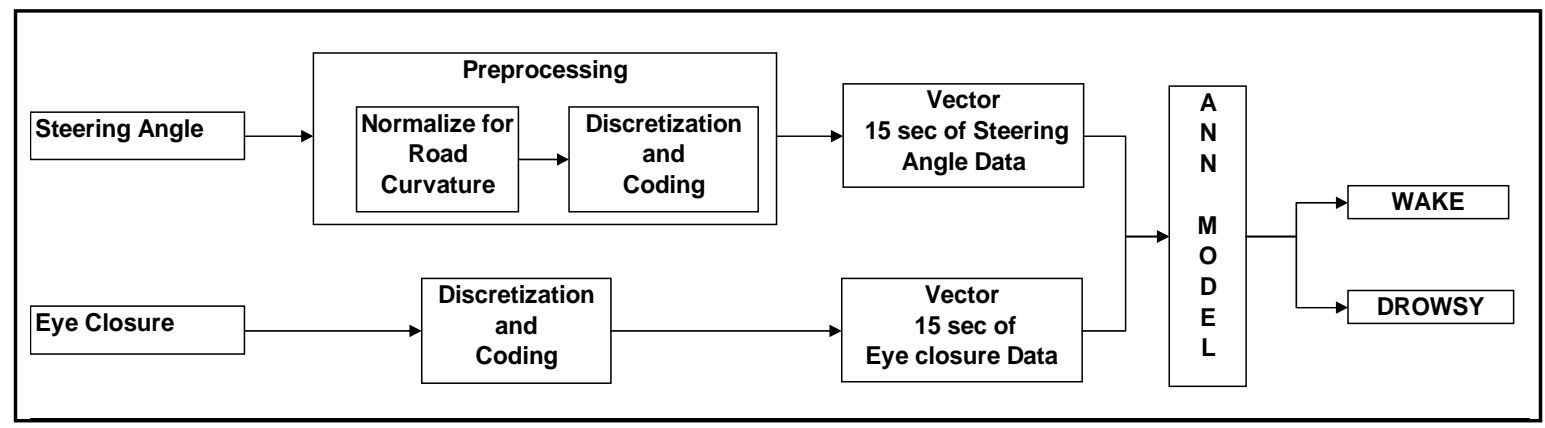

Figure 5.6: ANN model based on steering angle and eye closure 


\section{EYE CLOSURE DATA}

As explained earlier, the eye tracking system records the pupil diameter, and a zero value means that the eyelid is closed. The data was recorded at $60 \mathrm{~Hz}$ during the experiment. In order to match with the steering angle data, it was reduced to $1 \mathrm{~Hz}$, by using a new variable called $C_{i}$, where

$$
\mathbf{C}_{\mathbf{i}}=\text { Number of zeros in one second of data (60 data points). }
$$

This data, which now represent one second of eye closures, was used in further analysis. An example of a small portion of the eye closures data file is shown in Table 5.12.

\section{Input Discretization (Eye Data)}

$\mathrm{C}_{\mathrm{i}}$, which now represents one second of eye closures, was discretized into a fourdimension vector according to the following scheme:

Let $r_{9}, r_{10}, r_{11}$, and $r_{12}$ each represent a discrete range. These ranges are defined as follows:

$$
\sum_{\mathrm{k}=18-\mathrm{i}}^{9} \mathrm{p}_{\mathrm{k}} \leq \mathrm{r}_{\mathrm{i}}<\sum_{\mathrm{k}=17-\mathrm{i}}^{9} \mathrm{p}_{\mathrm{k}} \quad \text { for } \mathrm{i}=9 \rightarrow 12
$$

where $\mathrm{p}_{\mathrm{k}},(\mathrm{k}=5 \ldots 9)$ are constants.

These constants were used to define the discretization ranges. The blink rate (frequency of blinking) and blink duration (amount of time the eye is continuously closed) are highly dependent on individual behavior. The constants $\mathrm{p}_{\mathrm{k}}$ are used to accommodate the variations in blink rate and blink duration. Larger values of $p_{k}$ will make the discretization ranges wider to accommodate large duration and high frequency, while smaller values will make them shorter for low frequency and shorter duration blinks.

With:

$$
\begin{aligned}
& \mathrm{p}_{5}>60 \text { (upper limit is } 60 \mathrm{~Hz} \text { ) } \\
& \mathrm{p}_{6}, \mathrm{p}_{7} \text {, and } \mathrm{p}_{8} \text { are selected according to the driving behavior of individual driver } \\
& \mathrm{p}_{9}=0 \text { (no closures) }
\end{aligned}
$$

Table 5.11 shows these ranges, specifically for $\mathrm{p}_{6}=\mathrm{p}_{7}=\mathrm{p}_{8}=6$

Table 5.11: Ranges for $p_{6}=p_{7}=p_{8}=6$

\begin{tabular}{|c|c|}
\hline$\underline{\mathbf{r}}_{\mathbf{i}}$ & $\underline{\text { Range }}$ \\
\hline $\mathrm{r}_{9}$ & $0 \leq \mathrm{r}_{9}<6$ \\
\hline $\mathrm{r}_{10}$ & $6 \leq \mathrm{r}_{10}<12$ \\
\hline $\mathrm{r}_{11}$ & $12 \leq \mathrm{r}_{11}<18$ \\
\hline
\end{tabular}




\begin{tabular}{|l|l|}
\hline $\mathrm{r}_{12}$ & $18 \leq \mathrm{r}_{12}<78$ \\
\hline
\end{tabular}


Table 5.12: Example of Eye closure data

\begin{tabular}{|c|c|c|c|c|c|c|c|c|c|c|c|c|c|c|c|c|c|c|c|c|c|c|c|c|c|c|c|c|c|c|c|c|c|c|c|c|c|c|c|c|c|c|c|c|c|c|c|c|c|c|c|c|c|c|c|c|c|c|}
\hline $\begin{array}{c}\mathrm{T} \\
\text { sec }\end{array}$ & \multicolumn{57}{|c|}{ Pupil Diameter $(60 \mathrm{~Hz})$} & \\
\hline 1 & 92 & & 100 & 94 & 89 & 89 & 90 & 89 & 89 & 89 & 90 & 90 & 89 & 89 & 89 & 89 & 91 & 90 & 89 & 888 & 888 & \begin{tabular}{l|l}
89 & 88
\end{tabular} & 89 & 89 & 8988 & 88 & 88 & 878 & 878 & $37 \mid 8$ & 78 & 786 & 86 & 686 & 86 & 85 & & 86 & & 96 & 898 & 89 & 89 & 88 & 89 & 89 & 89 & 89 & 89 & 89 & 89 & 89 & 90 & 0 & 0 & 0 & 0 & 4 \\
\hline 2 & 0 & 0 & 0 & 0 & 0 & 78 & 89 & 90 & 91 & 92 & 91 & 92 & 92 & 92 & 92 & 93 & 92 & 95 & 93 & 94 & 94 & \begin{tabular}{l|l}
93 & 93
\end{tabular} & 93 & 96 & \begin{tabular}{l|l}
93 & 93
\end{tabular} & \begin{tabular}{l|l}
3 & 95 \\
\end{tabular} & 93 & & $93 \mid 9$ & 939 & 49. & $\begin{array}{ll}3 & 94\end{array}$ & 494 & \begin{tabular}{l|l}
4 & 99
\end{tabular} & 0 & 90 & 95 & 92 & 93 & 93 & 92 & 91 & 93 & 92 & 93 & 92 & 0 & 92 & 91 & 91 & 90 & 91 & 91 & 90 & 90 & 90 & 90 & 7 \\
\hline 3 & 90 & 88 & 89 & 89 & 89 & 89 & 88 & 89 & 89 & 88 & 88 & 89 & 89 & 88 & 88 & 88 & 88 & 88 & 88 & 88 & 90 & \begin{tabular}{l|l}
88 & 88
\end{tabular} & 88 & 88 & \begin{tabular}{l|l}
89 & 89
\end{tabular} & 989 & 89 & & \begin{tabular}{l|l|l}
88 & 8
\end{tabular} & \begin{tabular}{l|l|l}
39 & 9
\end{tabular} & 39 & 688 & 888 & 886 & 86 & 86 & 86 & 87 & 86 & 87 & & 86 & 86 & 86 & & 86 & 86 & 86 & 85 & 85 & 85 & 85 & 84 & 84 & 83 & 85 & 87 & 0 \\
\hline 4 & 81 & 81 & 81 & 81 & 81 & 80 & 80 & 0 & 80 & 80 & 79 & 0 & 0 & 0 & 0 & 0 & 0 & 0 & 0 & 79 . & 79 . & $\begin{array}{ll}79 & 80\end{array}$ & 0 & 80 & \begin{tabular}{l|l}
80 & 80
\end{tabular} & 080 & 80 & \begin{tabular}{l|l}
80 & 8
\end{tabular} & \begin{tabular}{l|l|l}
80 & 8
\end{tabular} & $\begin{array}{lll}30 & 8\end{array}$ & $\begin{array}{lll}0 & 8\end{array}$ & 080 & 080 & \begin{tabular}{l|l}
0 & 80
\end{tabular} & 80 & 80 & 80 & 81 & 80 & 81 & 80 & 0 & 0 & 0 & 0 & 0 & 0 & 0 & 0 & 0 & 80 & 80 & 80 & 81 & 80 & 0 & 81 & 18 \\
\hline 5 & 81 & 81 & 81 & 81 & 81 & 81 & 81 & 81 & 81 & 81 & 94 & 95 & 89 & 88 & 89 & 88 & 87 & 87 & 87 & 87 & 888 & \begin{tabular}{l|l}
87 & 87
\end{tabular} & 87 & 88 & 8993 & 388 & 87 & \begin{tabular}{|l|l}
87 & 8
\end{tabular} & 868 & \begin{tabular}{l|l}
37 & 8
\end{tabular} & \begin{tabular}{l|l|l}
6 & 8
\end{tabular} & 686 & 686 & 686 & 85 & 87 & 85 & 85 & 85 & 85 & 858 & 85 & 85 & 85 & 85 & 85 & 86 & 85 & 86 & 86 & 86 & 85 & 85 & 85 & 85 & 85 & 86 & 0 \\
\hline 6 & 86 & 86 & 85 & 85 & 85 & 87 & 85 & 88 & 90 & 85 & 84 & 83 & 83 & 83 & 83 & 0 & 83 & 83 & 83 & 838 & 82 & \begin{tabular}{l|l|l}
83 & 82
\end{tabular} & 282 & 82 & \begin{tabular}{l|l}
81 & 80
\end{tabular} & \begin{tabular}{l|l|l|}
0 & 80
\end{tabular} & 80 & \begin{tabular}{l|l}
79 & 8
\end{tabular} & \begin{tabular}{l|l}
80 & 7
\end{tabular} & \begin{tabular}{l|l|l}
79 & 75
\end{tabular} & 97 & $\begin{array}{lll}9 & 83\end{array}$ & 376 & 677 & 76 & 76 & 76 & 76 & 76 & 76 & 767 & 76 & 76 & 75 & 76 & 76 & 76 & 76 & 76 & 76 & 77 & 76 & 76 & 77 & 77 & 77 & 77 & 1 \\
\hline 7 & 77 & 78 & 77 & 78 & 78 & 78 & 78 & 78 & 78 & 77 & 77 & 77 & 77 & 77 & 77 & 77 & 77 & 81 & 88 & 83 & 83 & \begin{tabular}{l|l|l}
82 & 82
\end{tabular} & 20 & 0 & \begin{tabular}{l|l}
0 & 0
\end{tabular} & 0 & 0 & \begin{tabular}{|l|l}
81 & 8
\end{tabular} & \begin{tabular}{l|l|l}
82 & 8
\end{tabular} & \begin{tabular}{l|l}
82 & 8
\end{tabular} & 28 & $\begin{array}{l}3 \\
3\end{array} 82$ & 282 & 82 & 82 & 81 & 82 & 82 & 82 & 82 & 82 & 82 & 82 & 81 & 82 & 88 & 88 & 78 & 76 & 76 & 76 & 76 & 76 & 76 & 75 & 75 & 75 & 6 \\
\hline 8 & 75 & 75 & 75 & 74 & 75 & 75 & 74 & 74 & 74 & 75 & 74 & 77 & 73 & 73 & 73 & 73 & 73 & 73 & 73 & 73 & 0 & \begin{tabular}{l|l|l}
73 & 73
\end{tabular} & 373 & 73 & \begin{tabular}{l|l}
75 & 74
\end{tabular} & $\begin{array}{l}474 \\
\end{array}$ & 74 & 747 & $74 \mid 7$ & $74 \mid 7$ & 47 & $\begin{array}{ll}475 \\
\end{array}$ & 575 & 575 & 75 & 75 & 76 & 75 & 76 & 76 & 76 - & 76 & 76 & 77 & 76 & 76 & 76 & 76 & 76 & 76 & 76 & 76 & 76 & 76 & 76 & 76 & 76 & 1 \\
\hline 9 & 76 & 76 & 76 & 77 & 76 & 0 & 76 & 76 & 77 & 77. & & 77 & 77 & 77 & 77 & 0 & 78 & 0 & 85 & 84 & 838 & \begin{tabular}{l|l|l}
82 & 82
\end{tabular} & 282 & 83 & \begin{tabular}{l|l|l}
82 & 82
\end{tabular} & 282 & 82 & \begin{tabular}{l|l}
85 & 8
\end{tabular} & \begin{tabular}{l|l|l}
82 & 8
\end{tabular} & \begin{tabular}{l|l|l}
83 & 8.
\end{tabular} & 38. & $\begin{array}{lll}3 & 83\end{array}$ & $\begin{array}{lll}3 & 83\end{array}$ & $\begin{array}{l}382 \\
\end{array}$ & 82 & 84 & 83 & 83 & 85 & 83 & 80 & 80 & 79 & 80 & 79 & 80 & 79 & 78 & 78 & 77 & 78 & 78 & 78 & 77 & 82 & 78 & 72 & 3 \\
\hline 10 & 72 & 71 & 71 & 71 & 71 & 71 & 71 & 71 & 70 & 71 & 71 & 70 & 71 & 71 & 73 & 72 & 72 & 72 & 72 & 72 & 72 & \begin{tabular}{l|l}
72 & 72
\end{tabular} & 272 & 72 & \begin{tabular}{l|l|l}
72 & 81
\end{tabular} & 180 & 78 & 777 & \begin{tabular}{l|l|l}
78 & 7
\end{tabular} & \begin{tabular}{l|l|l}
76 & 78
\end{tabular} & 877 & $\begin{array}{lll}7 & 77\end{array}$ & \begin{tabular}{l|l}
7 & 78
\end{tabular} & \begin{tabular}{l|l}
8 & 77
\end{tabular} & 78 & 78 & 78 & 78 & 78 & 79 & 80 & 79 & 80 & 79 & 80 & 79 & 80 & 80 & 80 & 80 & 80 & 80 & 81 & 85 & 78 & 78 & 77 & 0 \\
\hline 11 & 72 & 71 & 71 & 71 & 71 & 71 & 71 & 71 & 70 & 71 & 71 & 70 & 71 & 71 & 73 & 72 & 72 & 72 & 72 & 72 & 72 & \begin{tabular}{l|l}
72 & 72
\end{tabular} & 272 & 72 & \begin{tabular}{l|l|l}
72 & 81
\end{tabular} & 180 & 78 & 777 & \begin{tabular}{l|l|l}
78 & 7
\end{tabular} & \begin{tabular}{l|l|l}
76 & 7
\end{tabular} & 87 & $\begin{array}{lll}7 & 77\end{array}$ & $\begin{array}{ll}778 \\
\end{array}$ & 877 & 78 & 78 & 78 & 78 & 78 & 79 & 80 & 79 & 80 & 79 & 80 & 79 & 80 & 80 & 80 & 80 & 80 & 80 & 81 & 85 & 78 & 78 & 77 & 0 \\
\hline 12 & 72 & 71 & 71 & 71 & 71 & 71 & 71 & 71 & 70 & 71 & 71 & 70 & 71 & 71 & 73 & 72 & 72 & 72 & 72 & 72 & 72 & \begin{tabular}{l|l}
72 & 72
\end{tabular} & 272 & 72 & $\begin{array}{lll}72 & 81\end{array}$ & 180 & 78 & 777 & \begin{tabular}{l|l|l}
78 & 7
\end{tabular} & \begin{tabular}{l|l|l}
76 & 78
\end{tabular} & $87^{7}$ & $\begin{array}{lll}7 & 77\end{array}$ & $\begin{array}{l}778 \\
78\end{array}$ & 877 & 78 & 78 & 78 & 78 & 78 & 79 & 80 & 79 & 80 & 79 & 80 & 79 & 80 & 80 & 80 & 80 & 80 & 80 & 81 & 85 & 78 & 78 & 77 & 0 \\
\hline 13 & 72 & 71 & 71 & 71 & 71 & 71 & 71 & 71 & 70 & 71 & 71 & 70 & 71 & 71 & 73 & 72 & 72 & 72 & 72 & 72 & 72 & \begin{tabular}{l|l}
72 & 72
\end{tabular} & 272 & 72 & \begin{tabular}{l|l|l}
72 & 81
\end{tabular} & 180 & 78 & $\begin{array}{ll}77 & 7\end{array}$ & \begin{tabular}{l|l|l}
78 & 7
\end{tabular} & $76 \mid 78$ & 87 & 777 & 778 & \begin{tabular}{l|l}
8 & 77
\end{tabular} & 78 & 78 & 78 & 78 & 78 & 79 & 80 & 79 & 80 & 79 & 80 & 79 & 0 & 0 & 0 & 0 & 0 & 80 & 81 & 85 & 78 & 78 & 77 & 4 \\
\hline 14 & 72 & 71 & 71 & 71 & 71 & 71 & 71 & 71 & 70 & 71 & 71 & 70 & 71 & 71 & 73 & 72 & 72 & 72 & 72 & 72 & 72 & \begin{tabular}{l|l}
72 & 72
\end{tabular} & 272 & 72 & \begin{tabular}{l|l|l}
72 & 81
\end{tabular} & 180 & 78 & 777 & \begin{tabular}{l|l|l}
78 & 7
\end{tabular} & 7678 & 87 & 777 & \begin{tabular}{l|l|l}
7 & 78
\end{tabular} & 87 & 78 & 78 & 78 & 78 & 78 & 79 & 80 & 79 & 80 & 79 & 80 & 79 & 80 & 80 & 80 & 80 & 80 & 80 & 81 & 85 & 78 & 78 & 77 & 0 \\
\hline 15 & 72 & 71 & 71 & 71 & 71 & 71 & 71 & 71 & 70 & 71 & 71 & 70 & 7 & 71 & 73 & 7 & 72 & 72 & 72 & 72 & 72 & \begin{tabular}{l|l}
72 & 72
\end{tabular} & 272 & 72 & \begin{tabular}{l|l|l}
72 & 81
\end{tabular} & 10 & 0 & 0 & \begin{tabular}{l|l}
0 & 0
\end{tabular} & \begin{tabular}{l|l}
0 & 0
\end{tabular} & 0 & 77 & 78 & \begin{tabular}{l|l}
8 & 77
\end{tabular} & 78 & 78 & 78 & 78 & 78 & 79 & 80 & 79 & 80 & 79 & 80 & 79 & 80 & 80 & 8 & & 8 & 80 & 81 & 85 & 7 & 78 & 77 & 7 \\
\hline
\end{tabular}




\section{Input Coding (Eye Data)}

After defining the discrete ranges, eye data (which is now represented by $\mathrm{C}_{\mathrm{i}}$ ) was coded into a four-dimensional vector $E(T)$ as follows.

If the value of $C_{i}$ falls within the range represented by $r_{i}$, then the $i^{\text {th }}$ component of $E(T)$ was set equal to 1 , and the remaining three components were set equal to zero. For example, if $\mathrm{C}_{\mathrm{i}}=7$ and $\mathrm{p}_{6}=\mathrm{p}_{7}=\mathrm{p}_{8}=6$, then it will fall in the range represented by $\mathrm{r}_{10}(6$ $\leq \mathrm{r}_{9}<12$ ). Therefore, only the 2 nd component of $E(T)$ is 1 while all other components are zero, such as,

$$
E=\left[\begin{array}{l}
0 \\
1 \\
0 \\
0
\end{array}\right]
$$

Table 5.13 shows input discretization and coding for a set of $\mathrm{C}_{\mathrm{i}}$ values.

Table 5.13: Discretization and coding of eye closure data for $p_{2}=p_{3}=p_{4}=6$

\begin{tabular}{|c|c|c|c|c|c|}
\hline \multirow{2}{*}{$\begin{array}{c}\text { Time } \\
\text { T sec }\end{array}$} & \multirow{2}{*}{$\mathrm{Ci}$} & \multicolumn{4}{|c|}{$\boldsymbol{E}(\mathbf{T})=\left[\mathbf{e}_{\mathbf{1}}, \mathbf{e}_{\mathbf{2}}, \mathbf{e}_{\mathbf{3}}, \mathbf{e}_{\mathbf{4}}\right]$} \\
\cline { 3 - 6 } & & $\mathbf{e}_{\mathbf{1}}$ & $\mathbf{e}_{\mathbf{2}}$ & $\mathbf{e}_{\mathbf{3}}$ & $\mathbf{e}_{\mathbf{4}}$ \\
\hline 1 & 4 & 1 & 0 & 0 & 0 \\
\hline 2 & 7 & 0 & 1 & 0 & 0 \\
\hline 3 & 0 & 1 & 0 & 0 & 0 \\
\hline 4 & 18 & 0 & 0 & 1 & 0 \\
\hline 5 & 0 & 1 & 0 & 0 & 0 \\
\hline 6 & 1 & 1 & 0 & 0 & 0 \\
\hline 7 & 6 & 0 & 1 & 0 & 0 \\
\hline 8 & 1 & 1 & 0 & 0 & 0 \\
\hline 9 & 3 & 1 & 0 & 0 & 0 \\
\hline 10 & 0 & 1 & 0 & 0 & 0 \\
\hline 11 & 0 & 1 & 0 & 0 & 0 \\
\hline 12 & 0 & 1 & 0 & 0 & 0 \\
\hline 13 & 4 & 1 & 0 & 0 & 0 \\
\hline 14 & 0 & 1 & 0 & 0 & 0 \\
\hline 15 & 7 & 0 & 1 & 0 & 0 \\
\hline
\end{tabular}

\section{Input Data (Steering + Eye)}

The eight-dimensional steering angle vector $I(T)$ and the four-dimensional eye closure vector $E(T)$ were combined to form a twelve dimension vector $J(T)$ as shown in Table 5.14. After coding, vector $J(T)$ was summed over an interval of $n$ seconds to get the input vector $\mathbf{X}(\mathbf{n})$. Each input vector $\mathbf{X}(\mathbf{n})$ then represents $n$ seconds of driving interval.

$$
\mathbf{X}(\mathbf{n})=J(T)+J(T+1)+J(T+2)+\cdots \cdots \cdots \cdot \cdots(T+n-1)
$$


Table 5.14: Twelve dimension vector $\mathrm{J}(\mathrm{T})$

\begin{tabular}{|c|c|c|c|c|c|c|c|c|c|c|c|c|c|}
\hline \multicolumn{10}{|c|}{ X(n) } \\
\hline $\mathrm{x} 1$ & $\mathrm{x} 2$ & $\mathrm{x} 3$ & $\mathrm{x} 4$ & $\mathrm{x} 5$ & $\mathrm{x} 6$ & $\mathrm{x} 7$ & $\mathrm{x} 8$ & $\mathrm{x} 9$ & $\mathrm{x} 10$ & $\mathrm{x} 11$ & $\mathrm{x} 12$ & SLEEP & WAKE \\
\hline 2 & 2 & 3 & 0 & 3 & 2 & 3 & 0 & 11 & 3 & 1 & 0 & 0 & 1 \\
\hline 0 & 1 & 0 & 14 & 0 & 0 & 0 & 0 & 9 & 2 & 3 & 1 & 1 & 0 \\
\hline 2 & 0 & 5 & 4 & 3 & 1 & 0 & 0 & 0 & 1 & 5 & 9 & 1 & 0 \\
\hline 0 & 0 & 2 & 3 & 9 & 1 & 0 & 0 & 11 & 4 & 0 & 0 & 0 & 1 \\
\hline 0 & 0 & 0 & 10 & 5 & 0 & 0 & 0 & 11 & 3 & 1 & 0 & 0 & 1 \\
\hline 0 & 5 & 3 & 6 & 1 & 0 & 0 & 0 & 8 & 3 & 2 & 2 & 1 & 0 \\
\hline 1 & 4 & 1 & 3 & 4 & 0 & 1 & 1 & 7 & 3 & 2 & 3 & 1 & 0 \\
\hline 1 & 5 & 2 & 0 & 5 & 1 & 1 & 0 & 10 & 1 & 1 & 3 & 1 & 0 \\
\hline
\end{tabular}

Data was averaged for one second intervals. For $\mathrm{n}=15, \mathbf{X}(\mathbf{n})$ now represents 15 seconds of steering and eye closure activity. Table 5.15 shows a portion of the input file.

Table 5.15: Example of input file for ANN (eye+steering)

\begin{tabular}{|c|c|c|c|c|c|c|c|c|c|c|c|c|c|c|}
\hline \multirow{3}{*}{$\begin{array}{l}\text { Time } \\
\text { T sec }\end{array}$} & \multirow{3}{*}{$\mathbf{C i}$} & \multirow{3}{*}{$\begin{array}{c}\text { St. } \\
\text { angle }\end{array}$} & \multicolumn{12}{|c|}{$J(T)$} \\
\hline & & & \multicolumn{8}{|c|}{$I(T)=[i 1, i 2, \ldots \ldots \ldots \ldots . . . i 8]$} & \multicolumn{4}{|c|}{$E(T)$} \\
\hline & & & i1 & $I 2$ & i3 & i4 & i5 & i6 & i7 & i8 & e1 & $e 2$ & $e 3$ & e4 \\
\hline 1 & 4 & 1.1 & 0 & 0 & 0 & 0 & 0 & 1 & 0 & 0 & 1 & 0 & 0 & 0 \\
\hline 2 & 7 & -3.1 & 1 & 0 & 0 & 0 & 0 & 0 & 0 & 0 & 0 & 1 & 0 & 0 \\
\hline 3 & 0 & -2.2 & 0 & 1 & 0 & 0 & 0 & 0 & 0 & 0 & 1 & 0 & 0 & 0 \\
\hline 4 & 18 & 0.8 & 0 & 0 & 0 & 0 & 1 & 0 & 0 & 0 & 0 & 0 & 1 & 0 \\
\hline 5 & 0 & $\begin{array}{l}-1.7 \\
\end{array}$ & 0 & 0 & 1 & 0 & 0 & 0 & 0 & 0 & 1 & 0 & 0 & 0 \\
\hline 6 & 1 & 3 & 0 & 0 & 0 & 0 & 0 & 0 & 1 & 0 & 1 & 0 & 0 & 0 \\
\hline 7 & 6 & 1.1 & 0 & 0 & 0 & 0 & 0 & 1 & 0 & 0 & 0 & 1 & 0 & 0 \\
\hline 8 & 1 & $\begin{array}{l}-3.1 \\
\end{array}$ & 1 & 0 & 0 & 0 & 0 & 0 & 0 & 0 & 1 & 0 & 0 & 0 \\
\hline 9 & 3 & -2.2 & 0 & 1 & 0 & 0 & 0 & 0 & 0 & 0 & 1 & 0 & 0 & 0 \\
\hline 10 & 0 & 0.8 & 0 & 0 & 0 & 0 & 1 & 0 & 0 & 0 & 1 & 0 & 0 & 0 \\
\hline 11 & 0 & $\begin{array}{l}-1.7 \\
\end{array}$ & 0 & 0 & 1 & 0 & 0 & 0 & 0 & 0 & 1 & 0 & 0 & 0 \\
\hline 12 & 0 & 3 & 0 & 0 & 0 & 0 & 0 & 0 & 1 & 0 & 1 & 0 & 0 & 0 \\
\hline 13 & 4 & 0.8 & 0 & 0 & 0 & 0 & 1 & 0 & 0 & 0 & 1 & 0 & 0 & 0 \\
\hline 14 & 0 & $\begin{array}{l}-1.7 \\
\end{array}$ & 0 & 0 & 1 & 0 & 0 & 0 & 0 & 0 & 1 & 0 & 0 & 0 \\
\hline 15 & 7 & 3 & 0 & 0 & 0 & 0 & 0 & 0 & 1 & 0 & 0 & 1 & 0 & 0 \\
\hline \multicolumn{3}{|l|}{$\mathrm{X}(\mathrm{n})$} & 2 & 2 & 3 & 0 & 3 & 2 & 3 & 0 & 11 & 3 & 1 & 0 \\
\hline
\end{tabular}




\section{ANN ARCHITECTURE (EYE+STEERING)}

The combined ANN model was based on both steering angle and eye closure data. The network architecture is similar to that used in the steering only model with the following modifications:

- The input layer has 12 neurons corresponding to the input vector $\mathbf{X ( n )}$

- The hidden layer has 23 neurons.

\section{TRAINING DATA (EYE+STEERING)}

Data from eight subjects, which make a total of 1,600 driving intervals (as explained earlier), was randomized and then divided into three separate files (Table 5.16):

- Training file, which includes a set of 900 input-output exemplar vectors

- Cross-validation file, which includes a set of 150 input-output exemplar vectors

- Testing file, which includes a set of 550 input-output exemplar vectors

These files contain input vectors $\mathbf{X}(\mathbf{n})$ and output vectors $\mathbf{D ( n )}$, each representing 15 seconds of driving.

Table 5.16: Number of exemplars in each data set (eye+steering)

\begin{tabular}{|c|c|c|c|}
\hline Number of Weights & Training File & Cross Validation & Testing File \\
\hline 322 & 900 & 150 & 550 \\
\hline
\end{tabular}

\section{ANN TRAINING (EYE+STEERING)}

The training was performed in a similar way as explained earlier in the section on steering angle model. The same training parameters and cross validation techniques were used as that in the steering only model.

Table 5.17 shows the ANN training performance. Figure 5.7 is a graph of the Mean Square Error (MSE) for training and cross validation of the network and indicates the network performance during training. The smooth drop of MSE indicates successful training of the ANN. 
Table 5.17: ANN performance during training (eye+steering)

\begin{tabular}{|c|c|c|}
\hline Best Networks & Training & Cross Validation \\
\hline Epoch \# & 500 & 500 \\
\hline Minimum MSE & 0.0433 & 0.0407 \\
\hline Final MSE & 0.0433 & 0.0407 \\
\hline
\end{tabular}

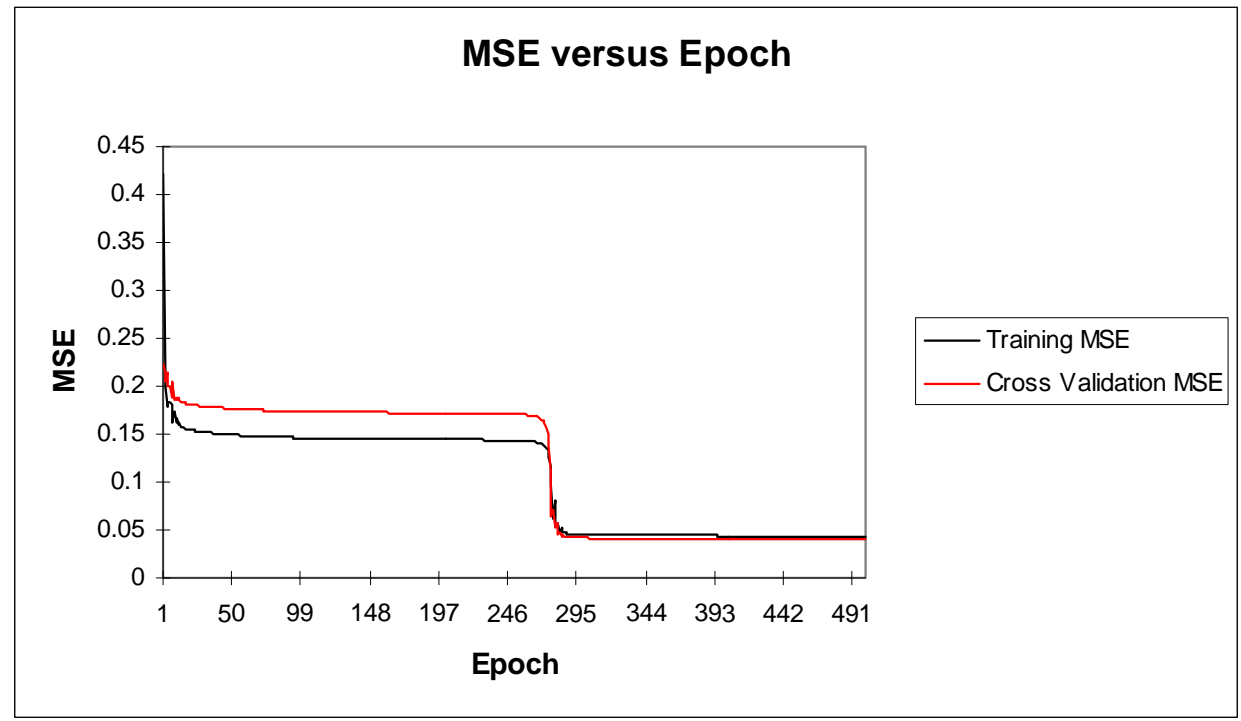

Figure 5.7: ANN training performance (eye+steering)

\section{ANN TESTING (EYE+STEERING)}

During the training process, ANN was tested after each iteration to test its performance. Table 5.18 shows network testing performance for the best network weights. The test data set (test file) contained 190 input vectors, each representing 15 seconds of driving. The test data was not used in training or cross validation (i.e., the network had never seen this data before).

Table 5.18: Test results (eye+steering)

\begin{tabular}{|c|c|c|}
\hline Output/Desired & AWAKE & DROWSY \\
\hline WAKE & 246 & 34 \\
\hline DROWSY & 24 & 246 \\
\hline \% CORRECT & 91 & 88 \\
\hline
\end{tabular}


The network correctly identified 246 out of a total of 280 drowsy intervals, an accuracy of $88 \%$. There were 24 "false alarms" in which intervals were in the awake class but were misclassified as drowsy. Also, 34 intervals that were in the drowsy class were misclassified by the network as awake.

\section{RESULTS AND DISCUSSION FOR TESTING ALL SUBJECTS}

Data from only eight subjects was used for training and initial testing. This data was mixed and randomized and did not represent a clear picture of driver behavior through the whole driving session. In this section, the results of the ANN models for all tested drivers during both morning and night sessions are presented. These results show a continuous picture of driver behavior throughout the driving sessions.

The data from both morning and night sessions was presented to the ANN for each of the driver. Overall, the performance of the drowsy driver detection systems was extremely good, as shown in Table 5.19. Both drowsy driver detection systems experienced some false alarms, in which the ANN misclassified some of the awake data as drowsy. Both systems produced an acceptable accuracy rate of between 85 and $88 \%$.

Table 5.19: False Alarm Rate and Accuracy

\begin{tabular}{|c|c|c|}
\hline & Steering & Steering +Eye \\
\hline False Alarms & $\mathbf{1 4 \%}$ & $\mathbf{9 \%}$ \\
\hline Accuracy & $\mathbf{8 5 \%}$ & $\mathbf{8 8} \%$ \\
\hline
\end{tabular}

Figures 5.8 through 5.20 show ANN outputs for all the subjects. It should be noted, because of the problems associated with the current eye tracker system, discussed in Eye Closure Data, some portions of the eye data are discarded from the results. These sections are hatched on the figures.

- The first graph is the area graph showing the fraction of time when the eye was closed in a 15 second time interval

- The second bar graph is output of the ANN, based on the steering only model

- The third bar graph is the output of the ANN, using both eye and steering angle data

In Figure 5.8, the x-axis for the ANN output represents 15 seconds time intervals. Each red bar means the ANN is classifying that particular interval as drowsy while the rest are classified as awake. 


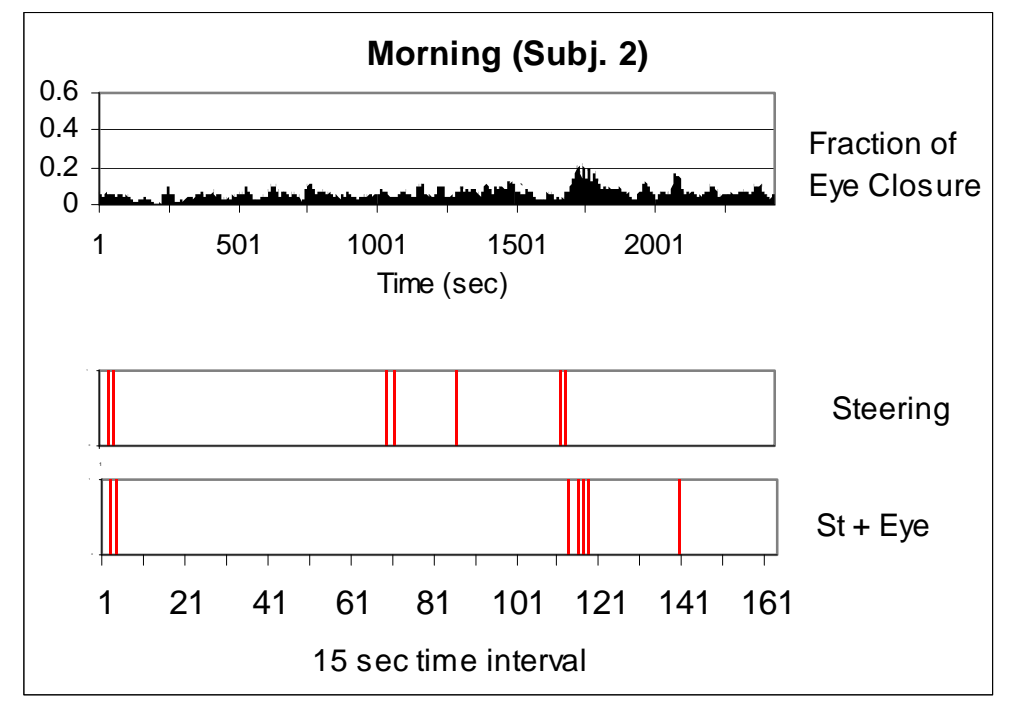

The first graph shows the fraction of the time that the eye was closed during a 15-second time interval. The second and third graph shows the ANN output for the steering only model and combined steering and eye model respectively. Each bar represents a 15 -second time interval that was classified as 'Drowsy' by the ANN.

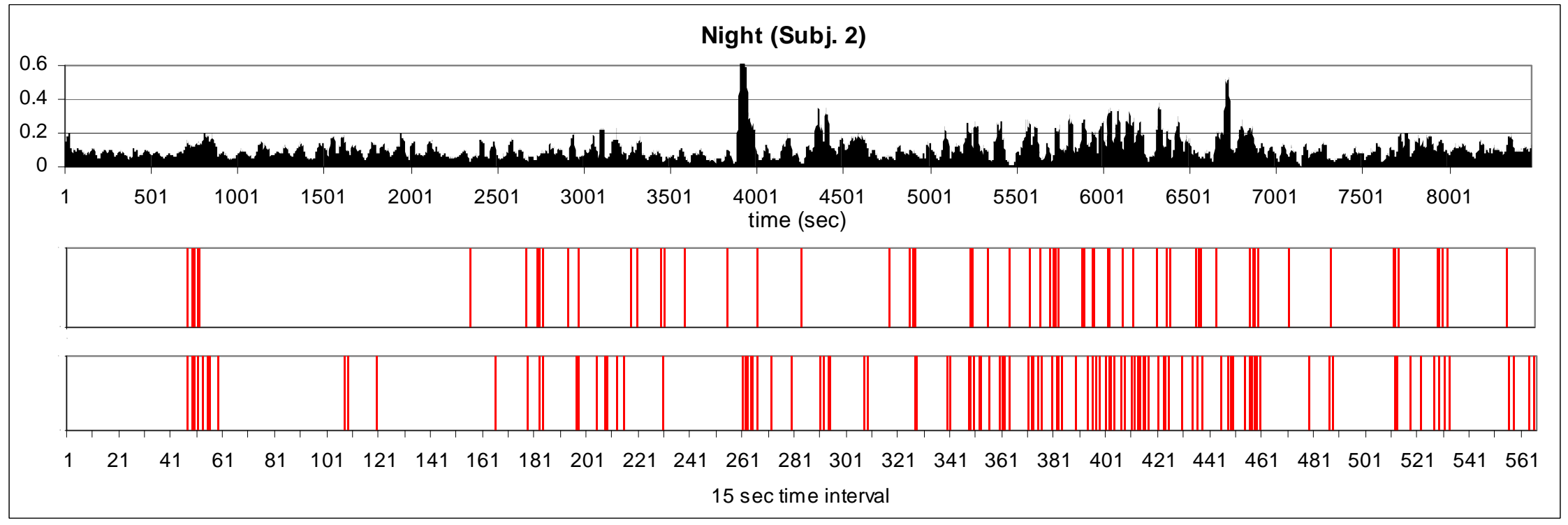

Figure 5.8: ANN output for subject 2 


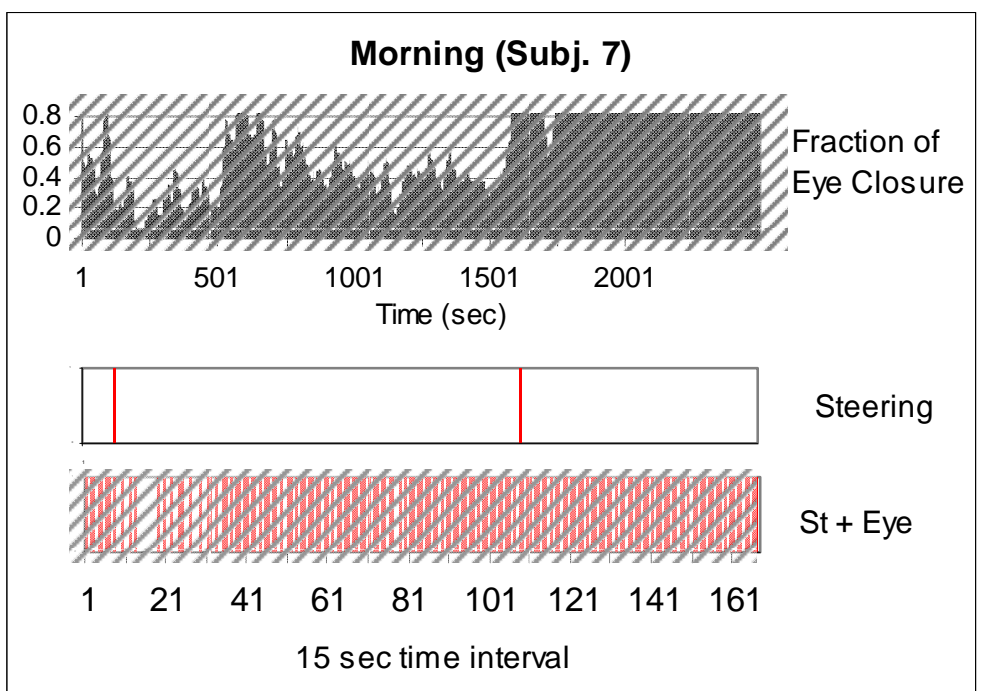

The first graph shows the fraction of the time that the eye was closed during a 15 -second time interval. The second and third graph shows the ANN output for the steering only model and combined steering and eye model respectively. Each bar represents a 15-second time interval that was classified as 'Drowsy' by the ANN.

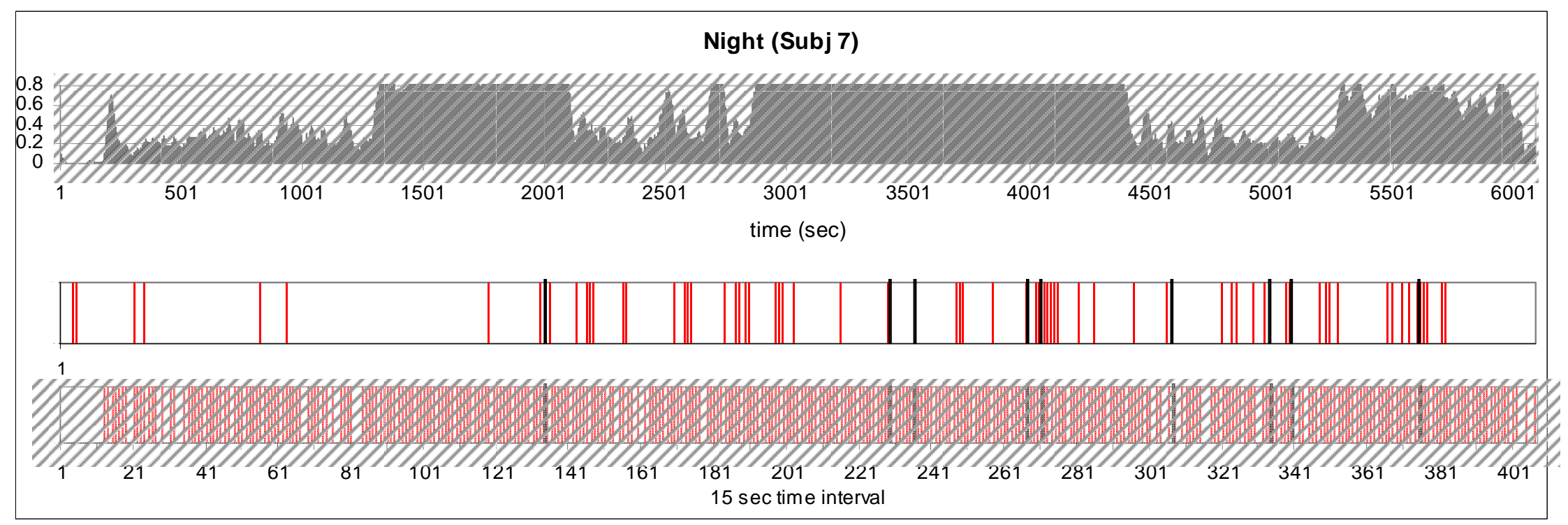

Figure 5.9: ANN output for subject 7 


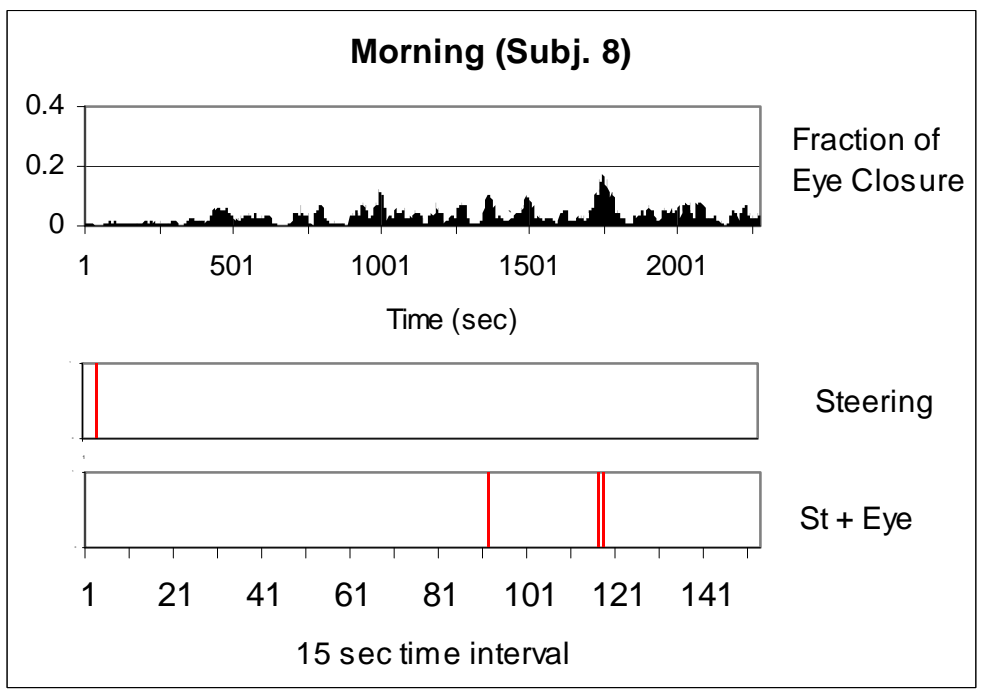

The first graph shows the fraction of the time that the eye was closed during a 15-second time interval. The second and third graph shows the ANN output for the steering only model and combined steering and eye model respectively. Each bar represents a 15 -second time interval that was classified as 'Drowsy' by the ANN.

\section{Night (Subj 8)}

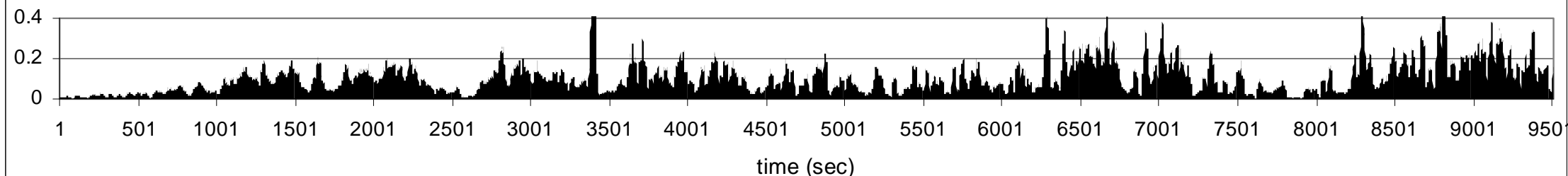

time $(\mathrm{sec})$

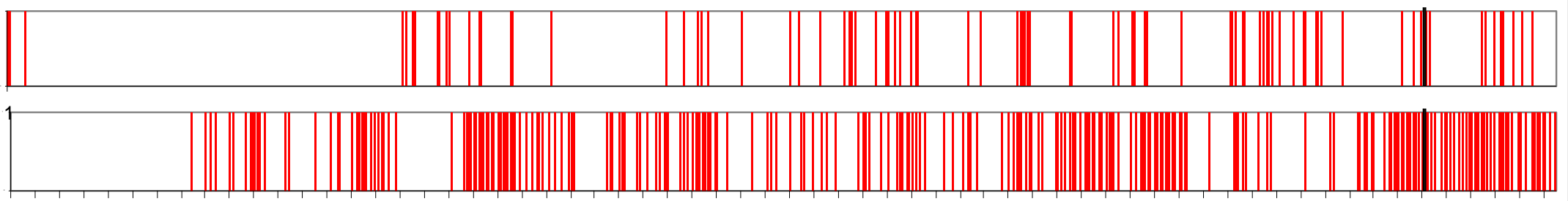

$1 \quad 2141 \quad 61 \quad 81101121141161181201221241261281301321341361381401421441461481501521541561581601621$ 15 sec time interval

Figure 5.10: ANN output for subject 8 


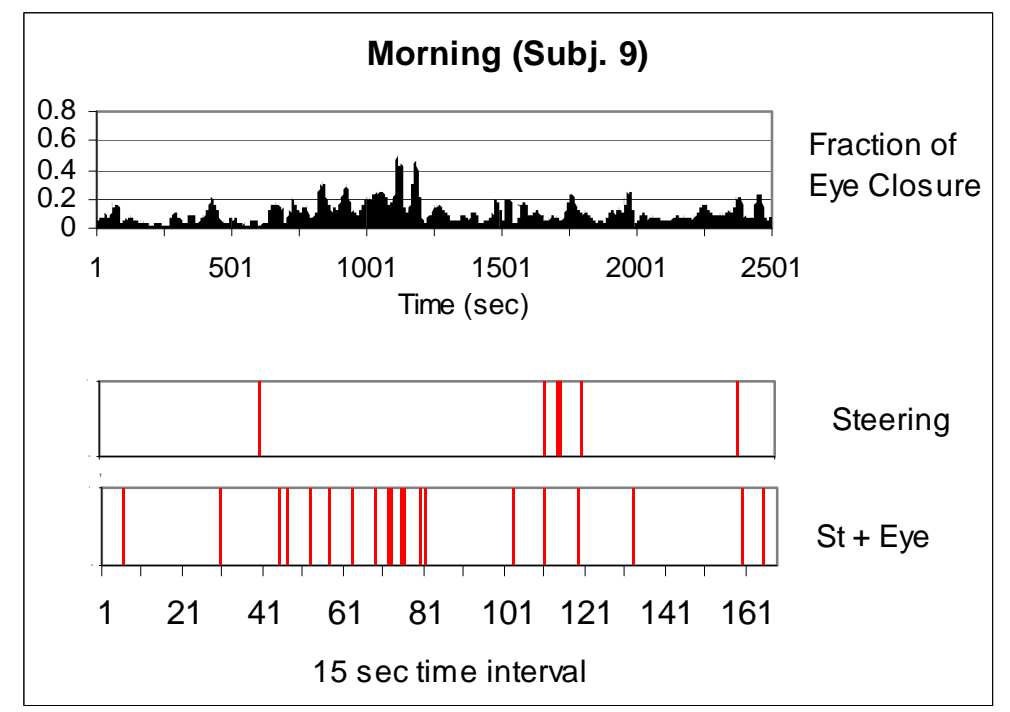

The first graph shows the fraction of the time that the eye was closed during a 15 -second time interval. The second and third graph shows the ANN output for the steering only model and combined steering and eye model respectively. Each bar represents a 15 -second time interval that was classified as 'Drowsy' by the ANN.

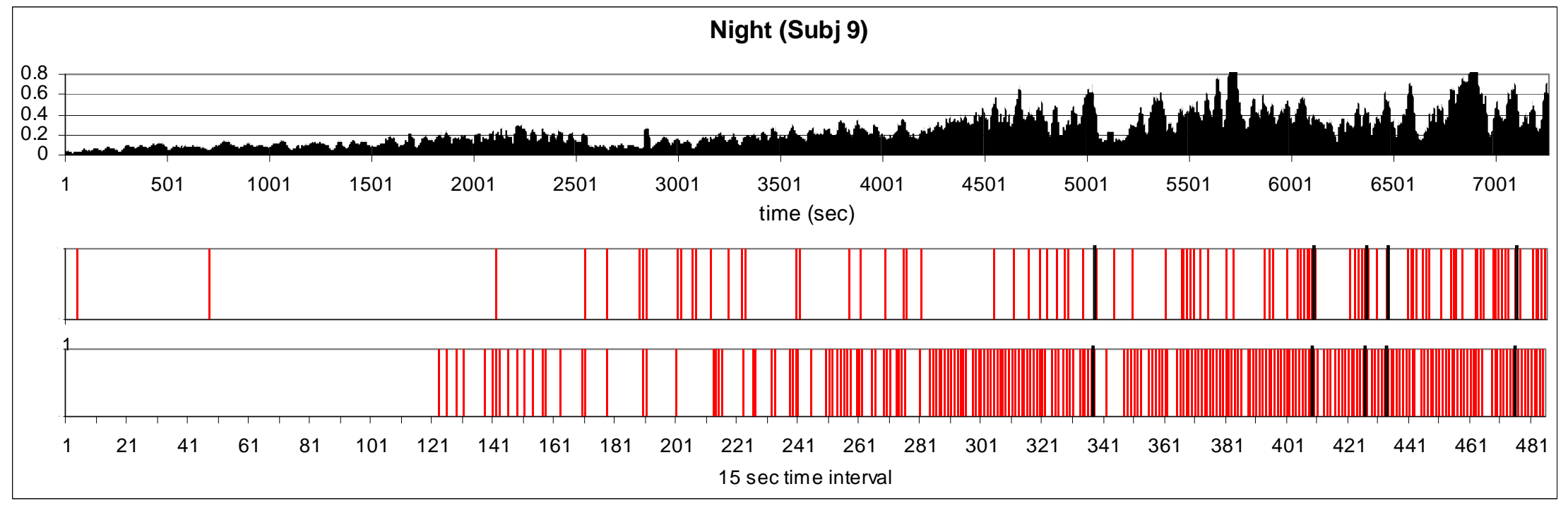

Figure 5.11: ANN output for subject 9 


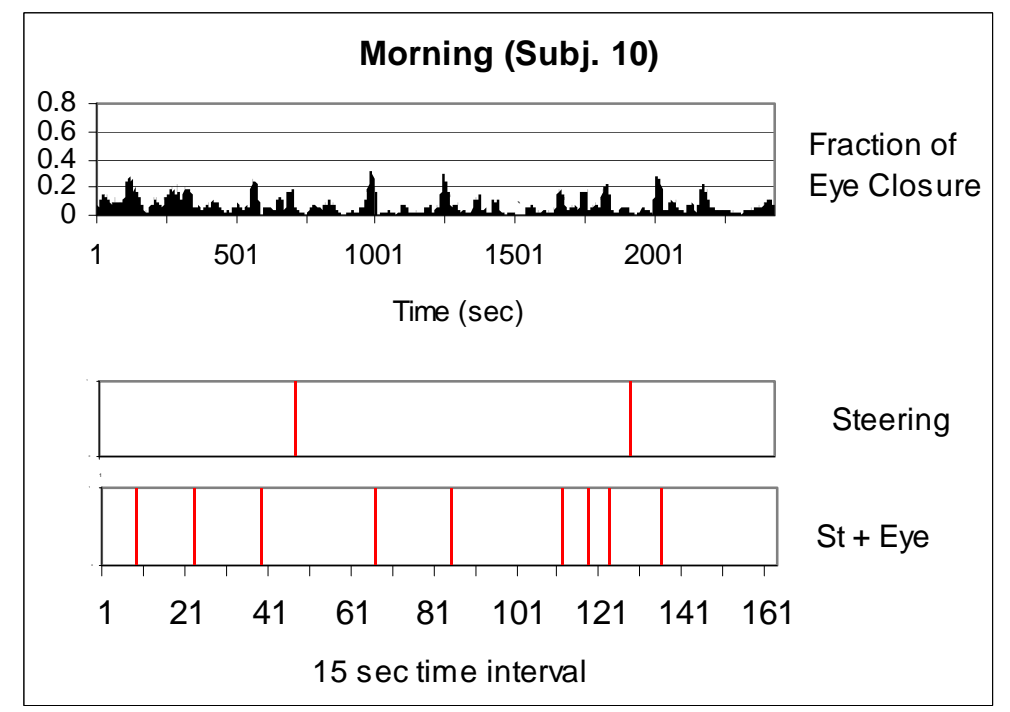

The first graph shows the fraction of the time that the eye was closed during a 15 -second time interval. The second and third graph shows the ANN output for the steering only model and combined steering and eye model respectively. Each bar represents a 15 -second time interval that was classified as 'Drowsy' by the ANN.

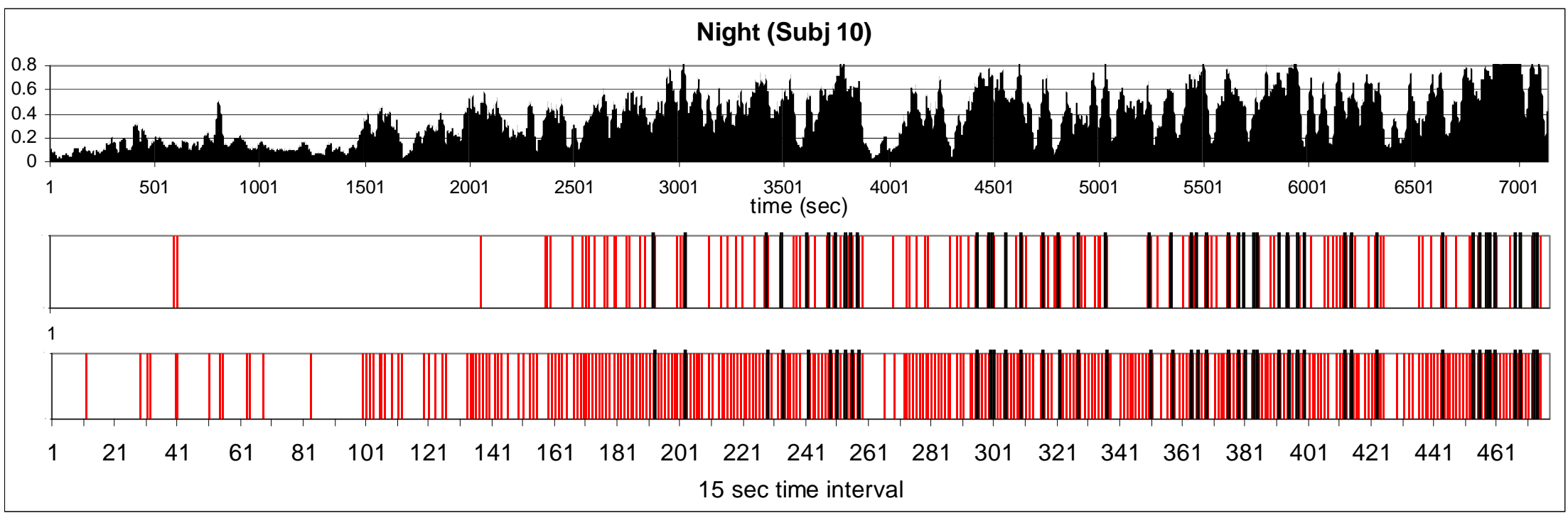

Figure 5.12: ANN output for subject 10 


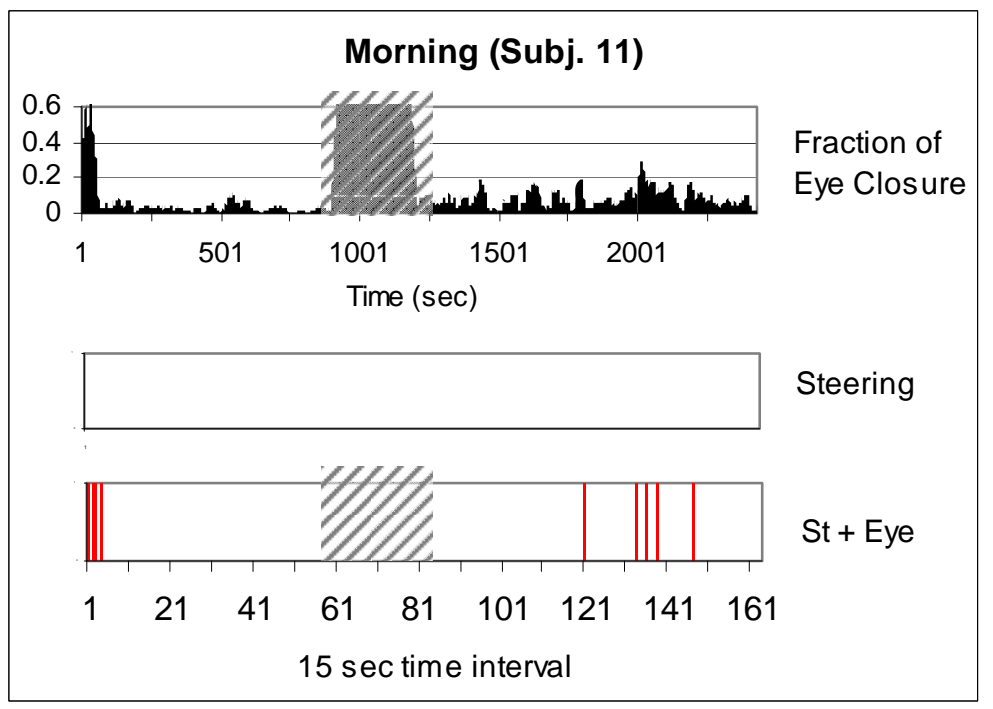

The first graph shows the fraction of the time that the eye was closed during a 15-second time interval. The second and third graph shows the ANN output for the steering only model and combined steering and eye model respectively. Each bar represents a 15 -second time interval that was classified as 'Drowsy' by the ANN.

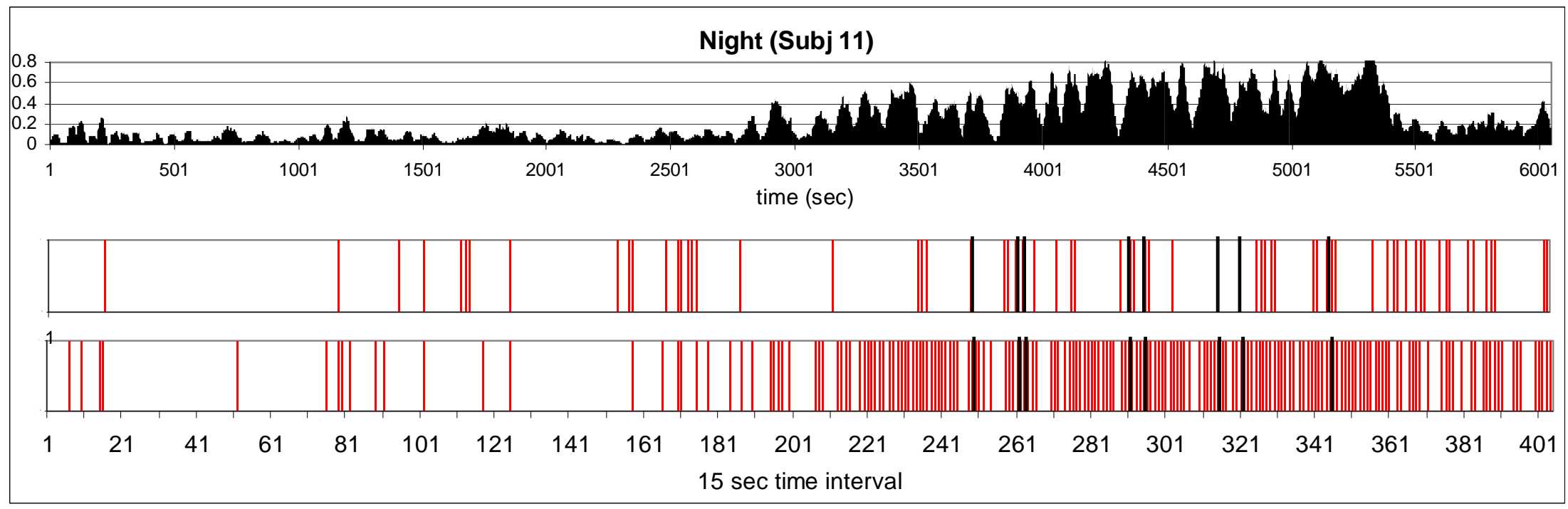

Figure 5.13: ANN output for subject 11 


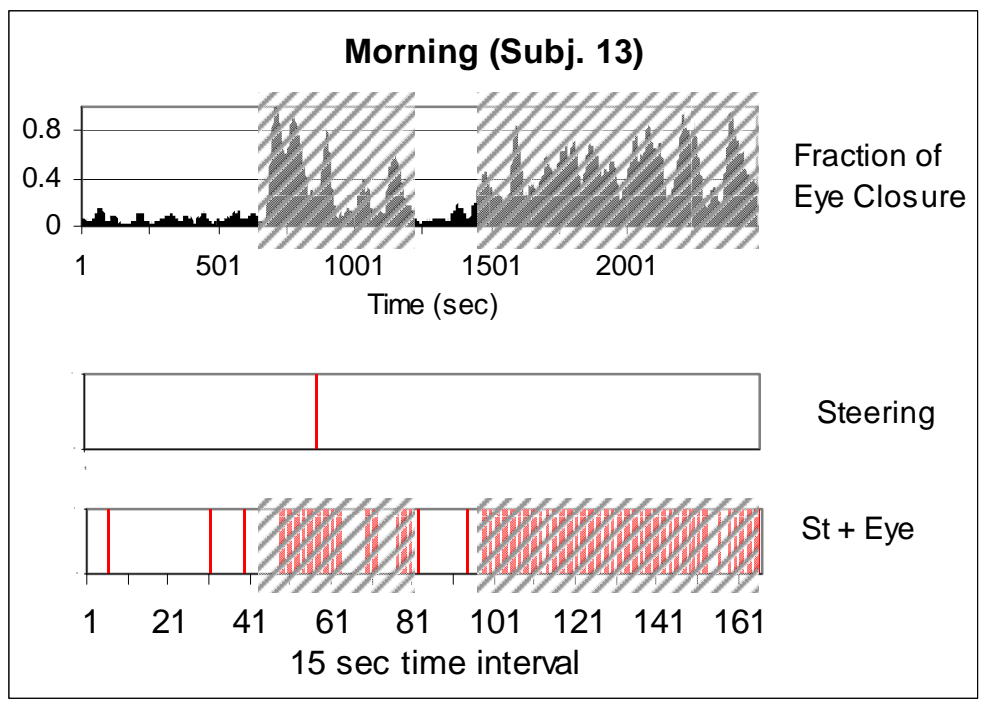

The first graph shows the fraction of the time that the eye was closed during a 15 -second time interval. The second and third graph shows the ANN output for the steering only model and combined steering and eye model respectively. Each bar represents a 15 -second time interval that was classified as 'Drowsy' by the ANN.

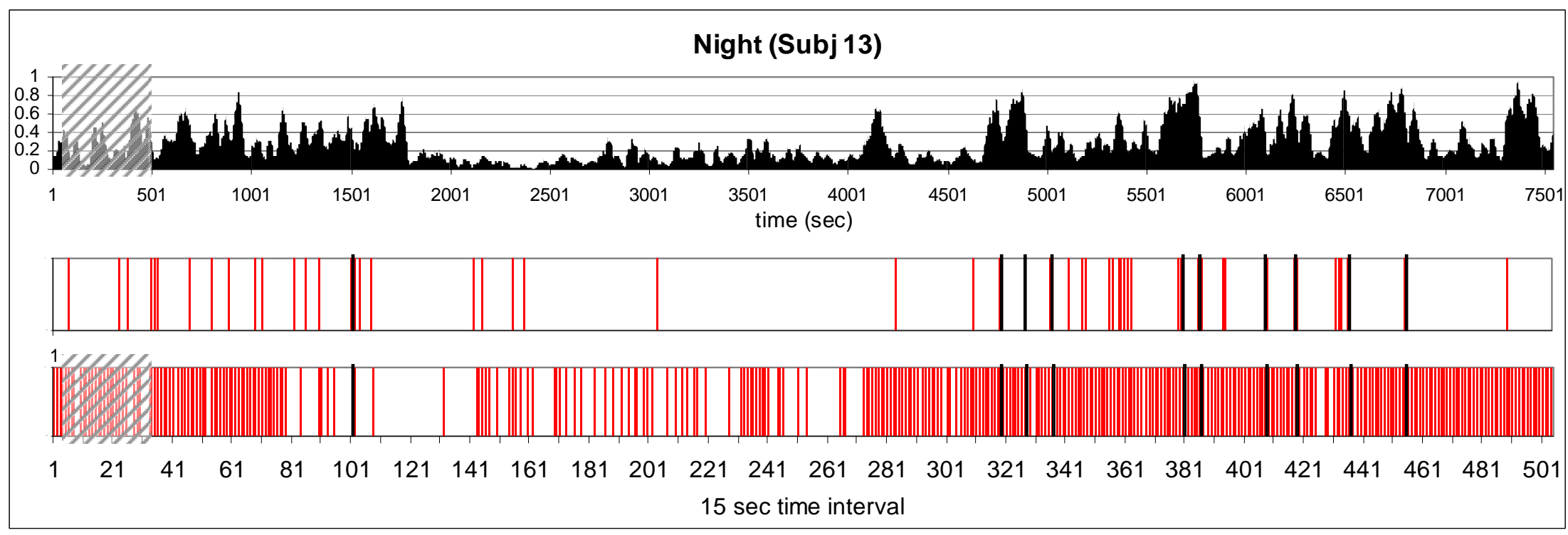

Figure 5.14: ANN output for subject 13 


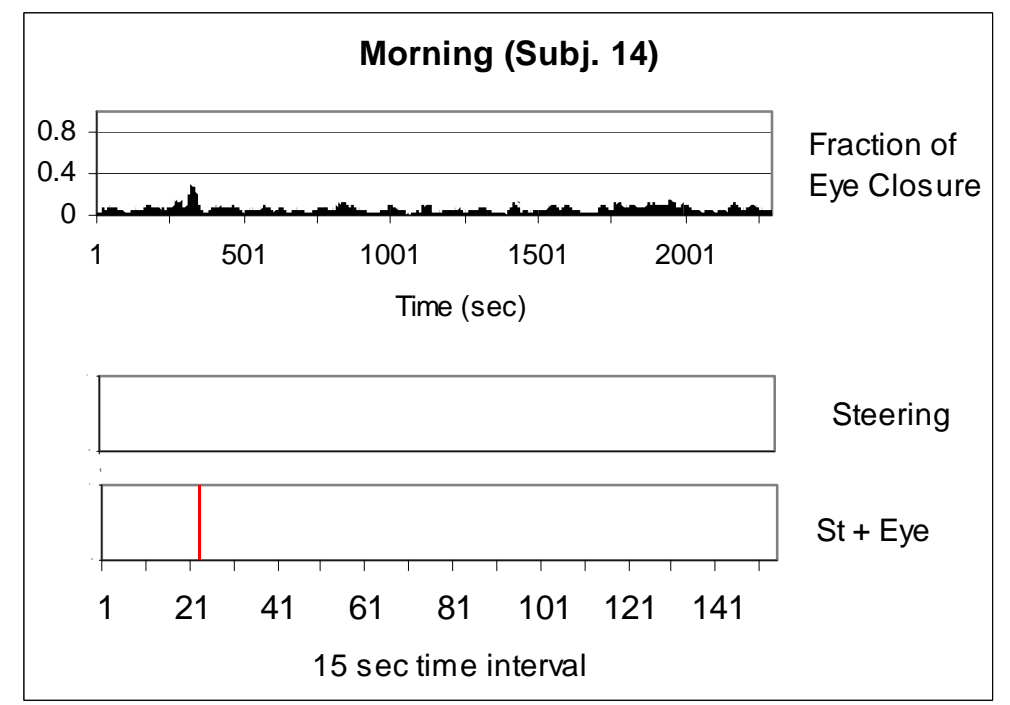

The first graph shows the fraction of the time that the eye was closed during a 15 -second time interval. The second and third graph shows the ANN output for the steering only model and combined steering and eye model respectively. Each bar represents a 15 -second time interval that was classified as 'Drowsy' by the ANN.

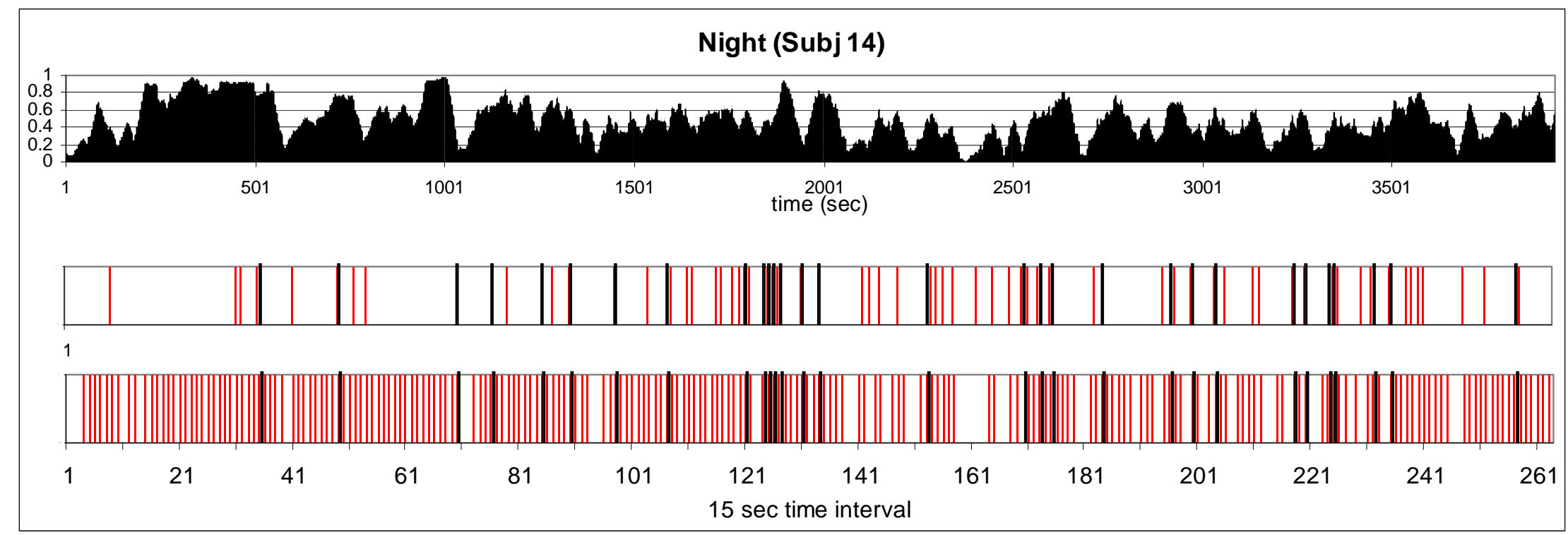

Figure 5.15: ANN output for subject 14 


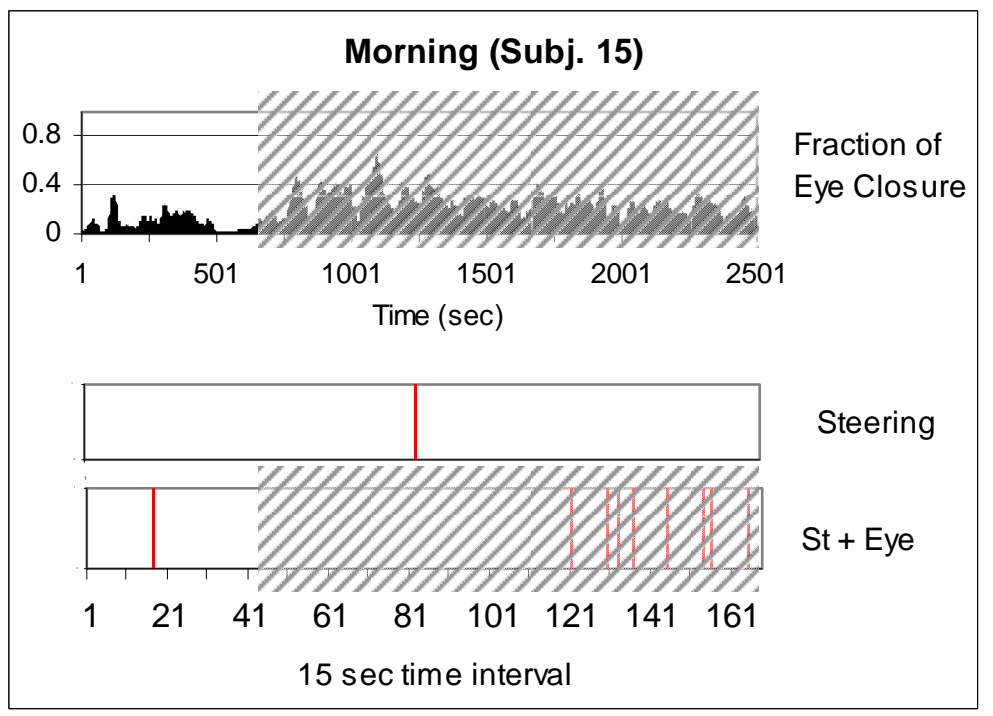

The first graph shows the fraction of the time that the eye was closed during a 15 -second time interval. The second and third graph shows the ANN output for the steering only model and combined steering and eye model respectively. Each bar represents a 15 -second time interval that was classified as 'Drowsy' by the ANN.

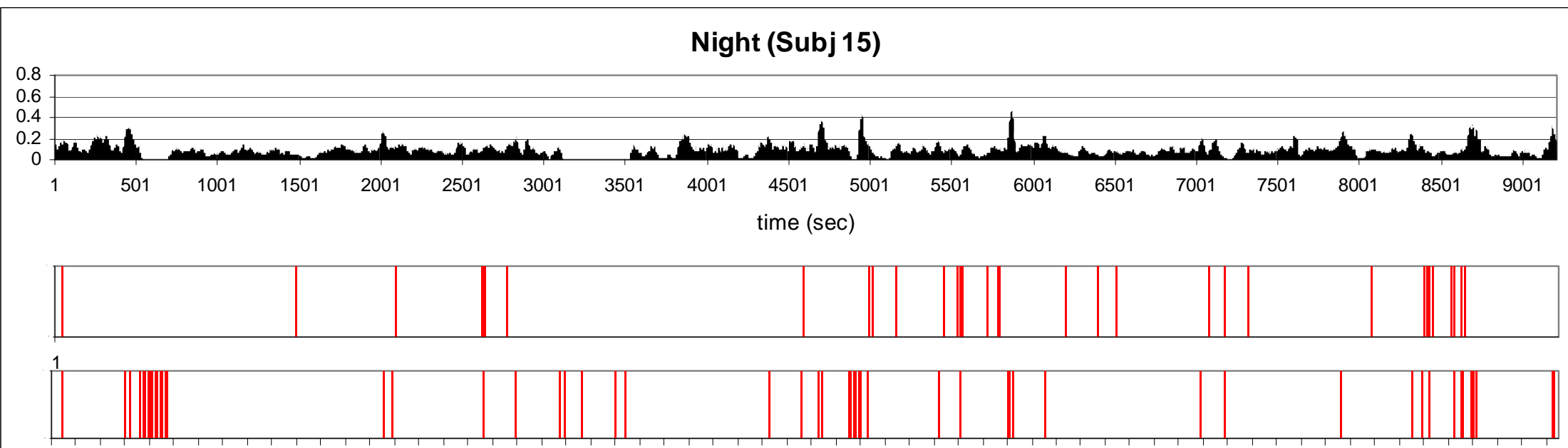

1214416181101121141161181201221241261281301321341361381401421441461481501521541561581601 $15 \mathrm{sec}$ time interval

Figure 5.16: ANN output for subject 15 


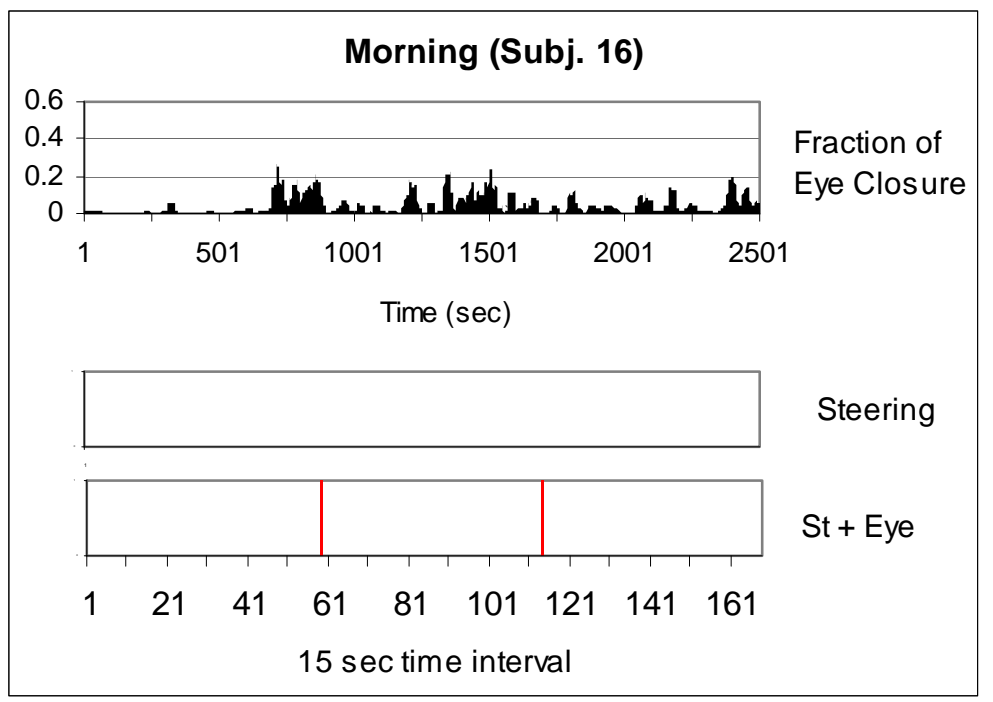

The first graph shows the fraction of the time that the eye was closed during a 15 -second time interval. The second and third graph shows the ANN output for the steering only model and combined steering and eye model respectively. Each bar represents a 15 -second time interval that was classified as 'Drowsy' by the ANN.

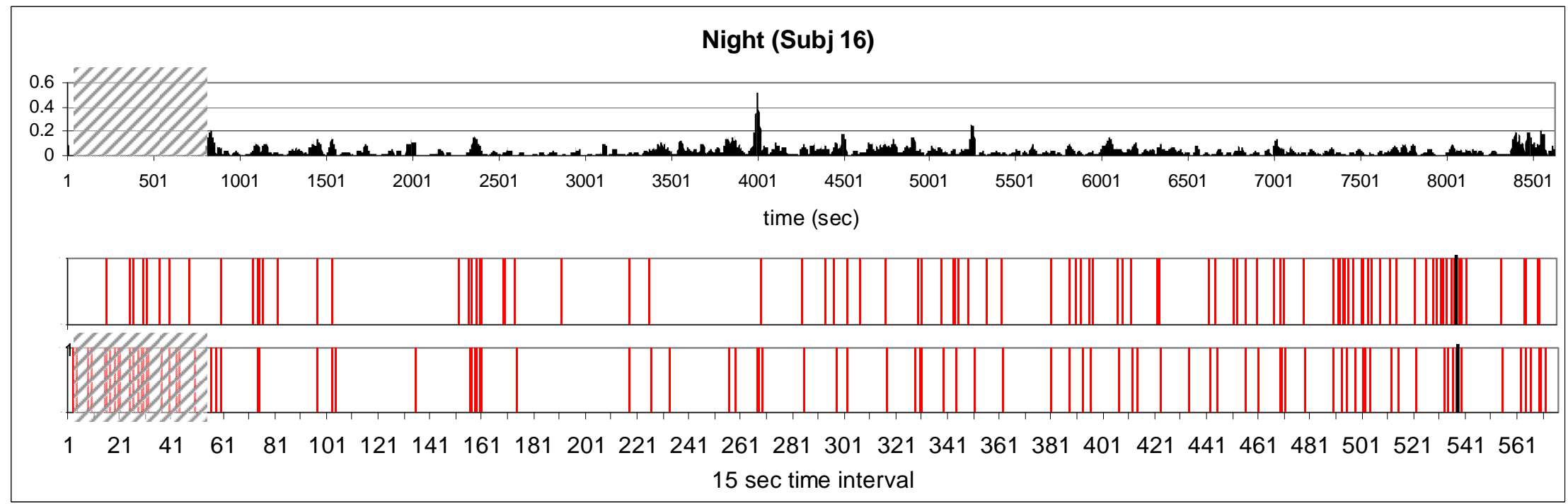

Figure 5.17: ANN output for subject 16 


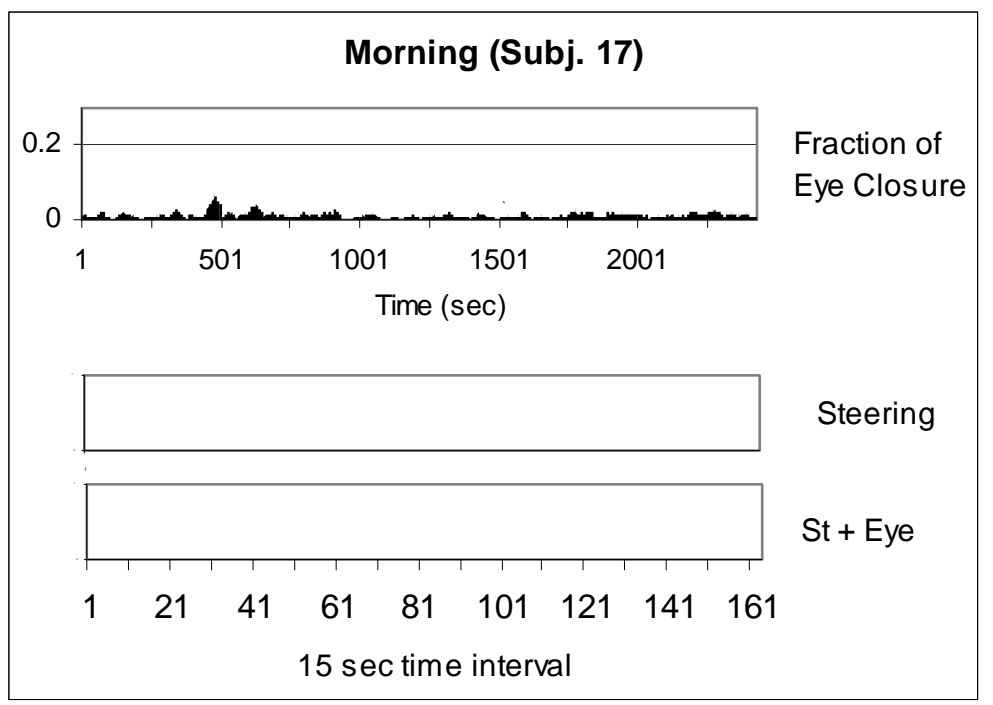

The first graph shows the fraction of the time that the eye was closed during a 15 -second time interval. The second and third graph shows the ANN output for the steering only model and combined steering and eye model respectively. Each bar represents a 15 -second time interval that was classified as 'Drowsy' by the ANN.

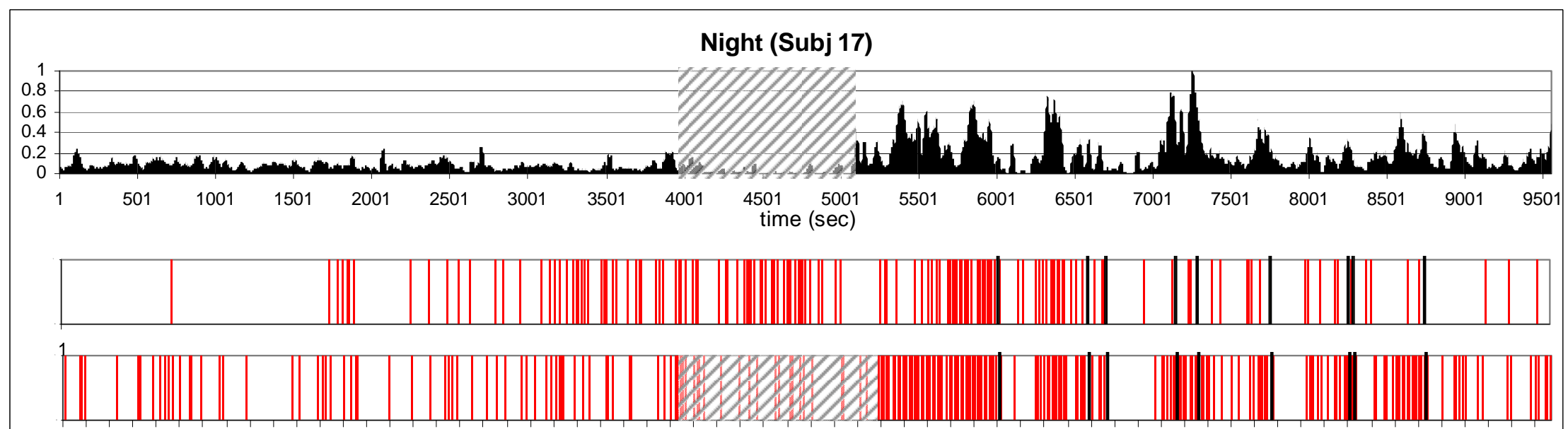

121416181101121141161181201221241261281301321341361381401421441461481501521541561581601621 15 sec time interval

Figure 5.18: ANN output for subject 17 


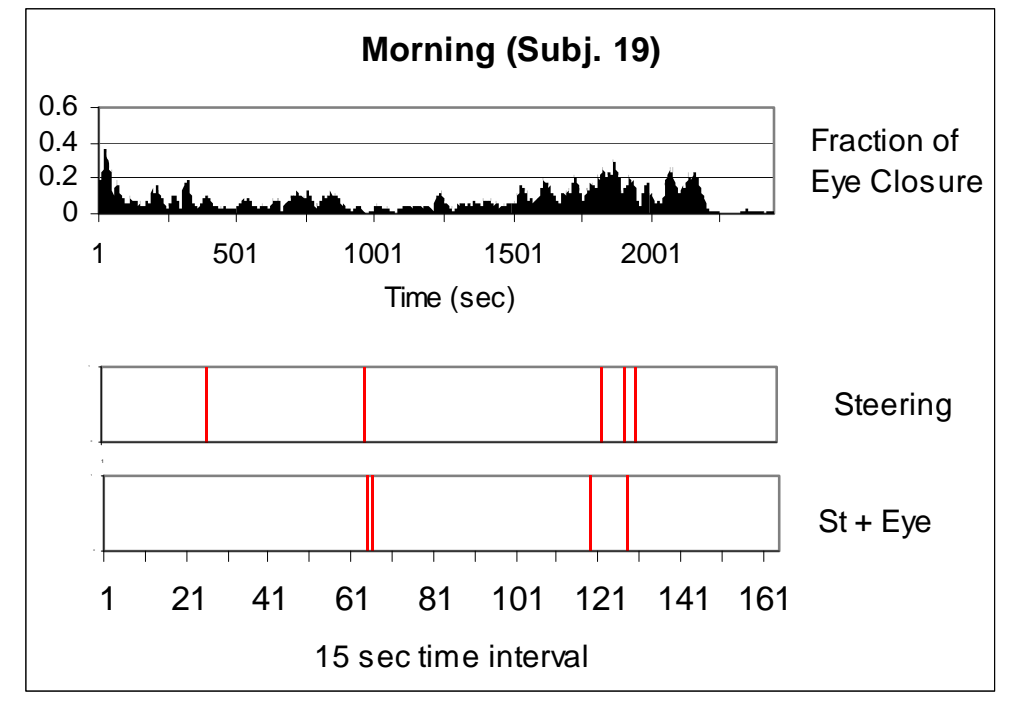

The first graph shows the fraction of the time that the eye was closed during a 15 -second time interval. The second and third graph shows the ANN output for the steering only model and combined steering and eye model respectively. Each bar represents a 15 -second time interval that was classified as 'Drowsy' by the ANN.

\section{Night (Subj 19)}

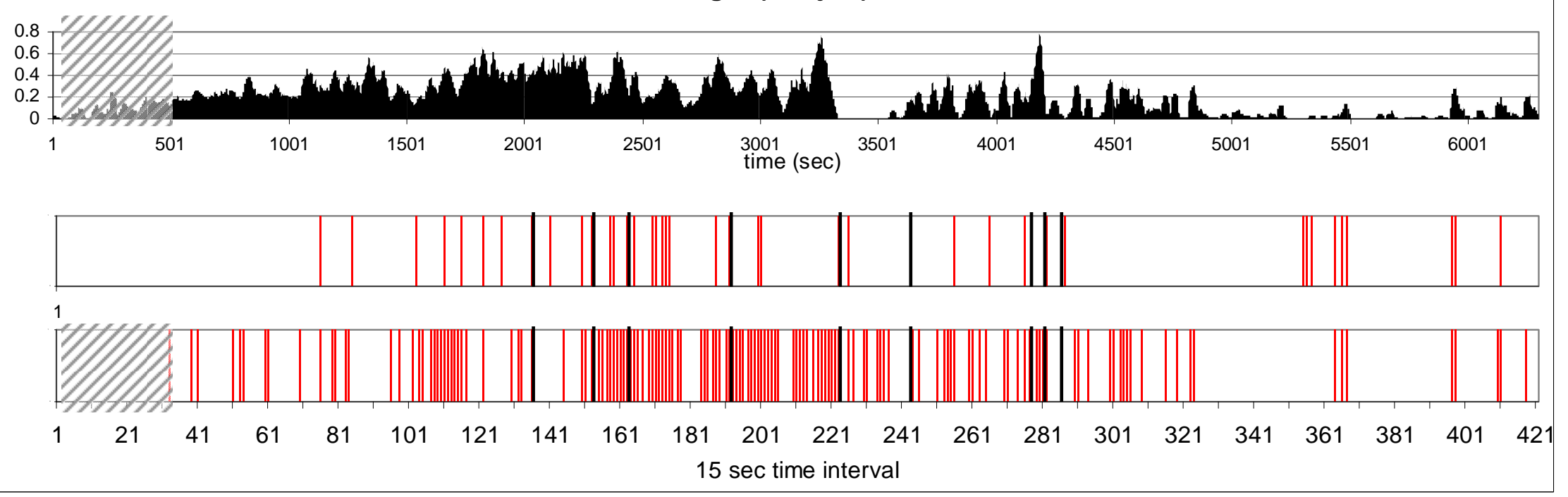

Figure 5.19: ANN output for subject 19 


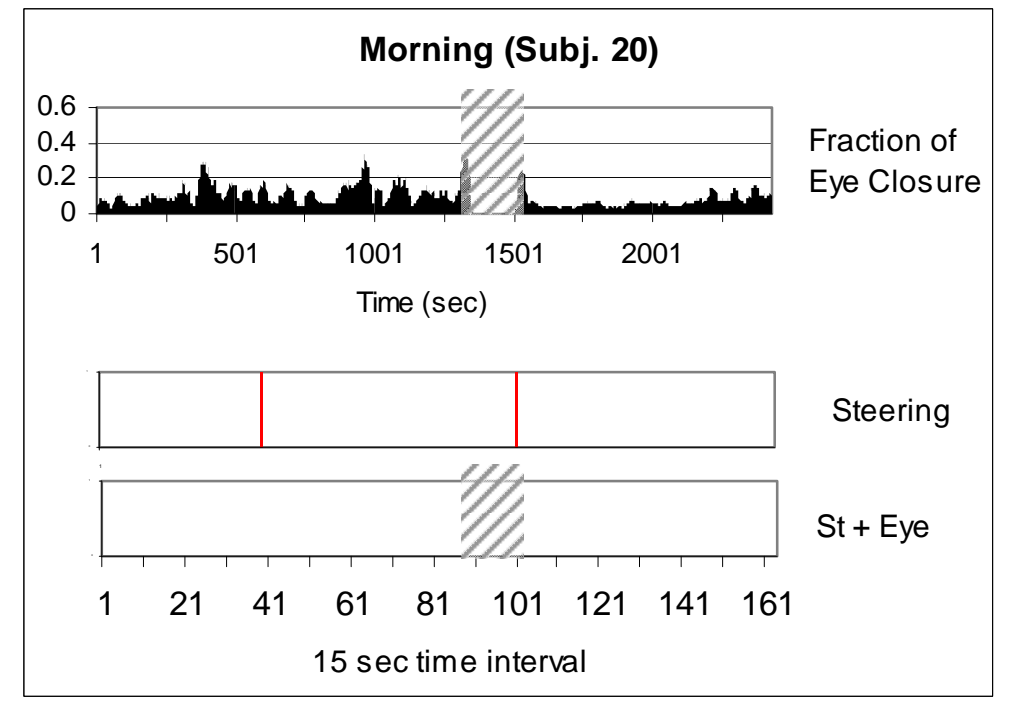

The first graph shows the fraction of the time that the eye was closed during a 15 -second time interval. The second and third graph shows the ANN output for the steering only model and combined steering and eye model respectively. Each bar represents a 15 -second time interval that was classified as 'Drowsy' by the ANN.

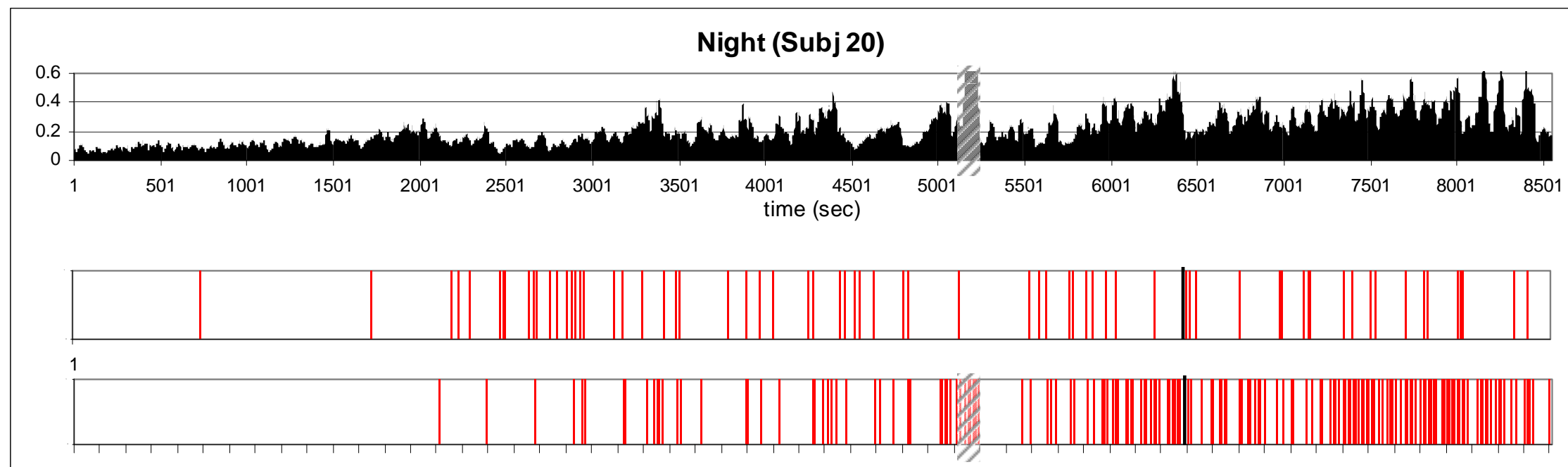

$1 \quad 2144161 \quad 81101121141161181201221241261281301321341361381401421441461481501521541561$ $15 \mathrm{sec}$ time interval

Figure 5.20: ANN output for subject 20 
The previous figures show that during the morning session most of the time the ANN output consists of awake intervals. The ANN also output few false alarms (i.e., intervals in the morning session were classified as drowsy). During the night session, a much higher number of intervals were classified as drowsy. In general, this trend is true for both ANN models.

There are very few false alarms in the steering only model output. On the average, only $1.5 \%$ of the morning data was classified as drowsy, less than three for close to 50 miles of driving. This is in a range which is quite manageable. If the system is set such that the actual alarm will be issued only if there are two or more consecutive 'Drowsy' outputs, this problem can be completely eliminated without sacrificing the system detection performance. For the night session, $16 \%$ of the steering only ANN output was classified as drowsy, which is not equally spread along the whole session but rather clustered in certain regions. Figure 5.21 shows this classification for the steering only ANN model.

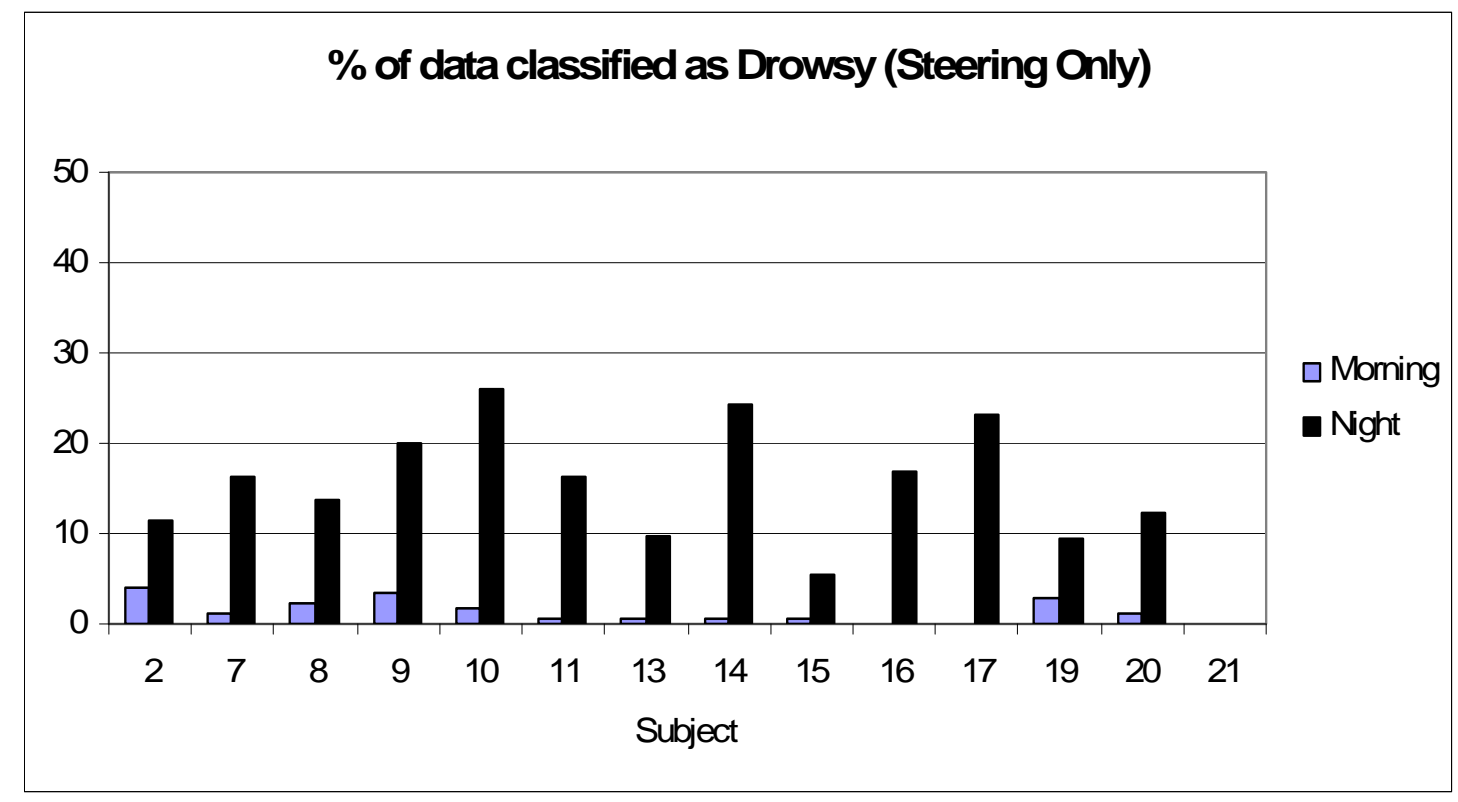

Figure 5.21: Percentage of 'Drowsy' intervals in morning versus night sessions (steering only)

When eye closure data was added to the model, it played a dominant role as is clear from Figures 5.8 - 5.20. The output of the ANN model that includes both steering and eye data shows a higher number of false alarms (i.e., intervals in the morning session that were classified as drowsy). Also, a much higher number of night session data was classified as drowsy. Figure 5.22 shows the percentage of ANN output that is classified as drowsy during both the morning and night sessions. On average, 12\% of morning session and $42 \%$ of night session data was classified as drowsy. 


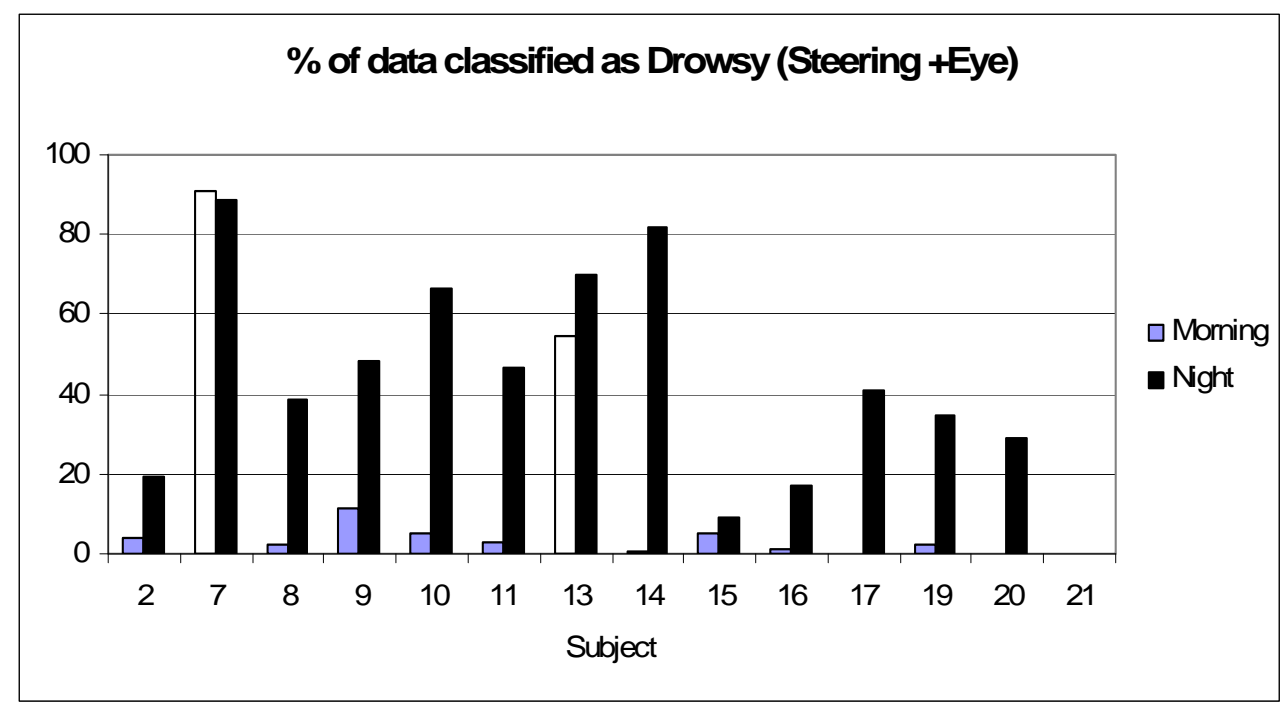

Figure 5.22: Percentage of 'Drowsy' in morning versus night sessions (steering+eye)

As explained in the description of data collection in Chapter 4 of this report, there were problems with capturing eye closure data for some subjects. The eye tracking system was not able to properly track the pupil diameter due to the type of glasses subjects were wearing. Also, data from the night session of subject, 10 and 14 recorded unusual amount of eye closures due to system misalignment by the subjects when they scratched their face or rubbed their eyes. If this data is removed, the percentage of false alarms drops from $12 \%$ to $3 \%$ and the drowsy intervals for night session from $42 \%$ to $30 \%$. These high percentages are a consequence of improper data recording or limitation of the technology and not of efficiency of the ANN model. For subjects whose eye data was recorded properly, the ANN output was very similar to that of the steering model.

Except for the subjects mentioned above, the output of both the ANN models correlates very well with the eye closure data. Figures $5.8-5.20$ show that although there is no one to one correlation in all areas of high closure activity, the ANN output of both ANN models shows dense clusters of drowsy output every time there is a high intensity of eye closures.

Figure 5.23 shows the average five minute PERCLOS measures for all drivers. Subjects whose eye data was properly recorded show an increase in average PERCLOS from $30 \%$ to $300 \%$ between morning and night sessions. Subjects 15 and 16 actually show a decrease in the average values of this measure but a look at the full data show short durations of high eye closures followed by long durations of low closures. The morning session was shorter than night session, which caused the average to fall. 


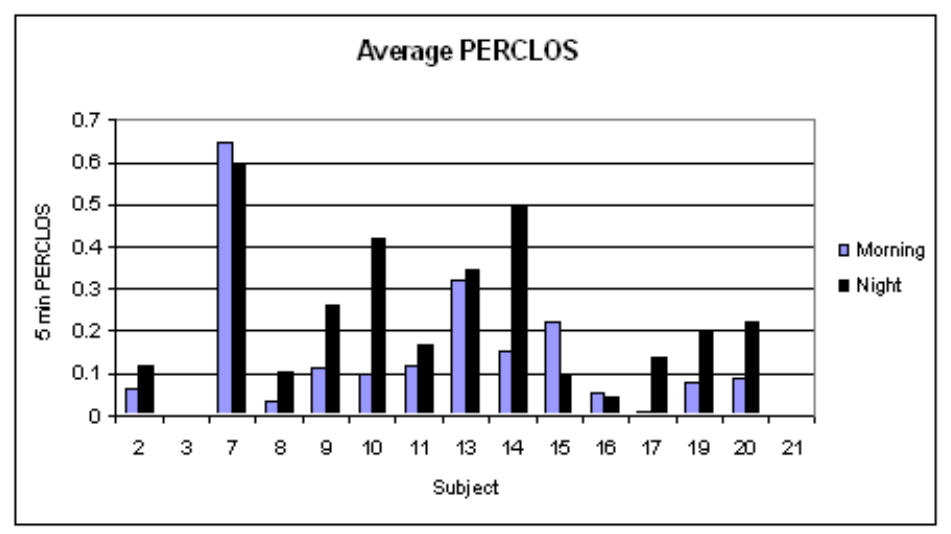

Figure 5.23: Average five-minute PERCLOS

Figure 5.24 shows a comparison of PERCLOS and ANN output of the steering model for six subjects. There is no one to one correlation between the two but it can be clearly seen that a segment of high eye closure activity is either followed or preceded by clusters of drowsy warnings.

In conclusion, the eye closures provide a good measure of driver drowsiness and can be used as a detection method. Systems used for eye closure monitoring do not work properly in all situations. The problems with the accurate detection of eye closures arise for a variety of reasons. It is highly dependent on individual behavior, face and eye structure, glasses, lighting conditions, and the system being used. It works for some individuals while completely failing for others. The technology is still not up to the task of detecting eye closures under all driving conditions unobtrusively. The eye tracking systems fails during the time when it is critically needed (i.e., when the driver is drowsy and is moving his head or rubbing his eyes). Unless these problems are solved, the model based only on eye closures will not be sufficient in itself.

Eye closure data dominate the model output in a combined steering and eye closure system. It is because of this dominance that the combined model did not perform well for some drivers due to the shortcomings in an eye monitoring system but the steering model performed well in a majority of cases. 


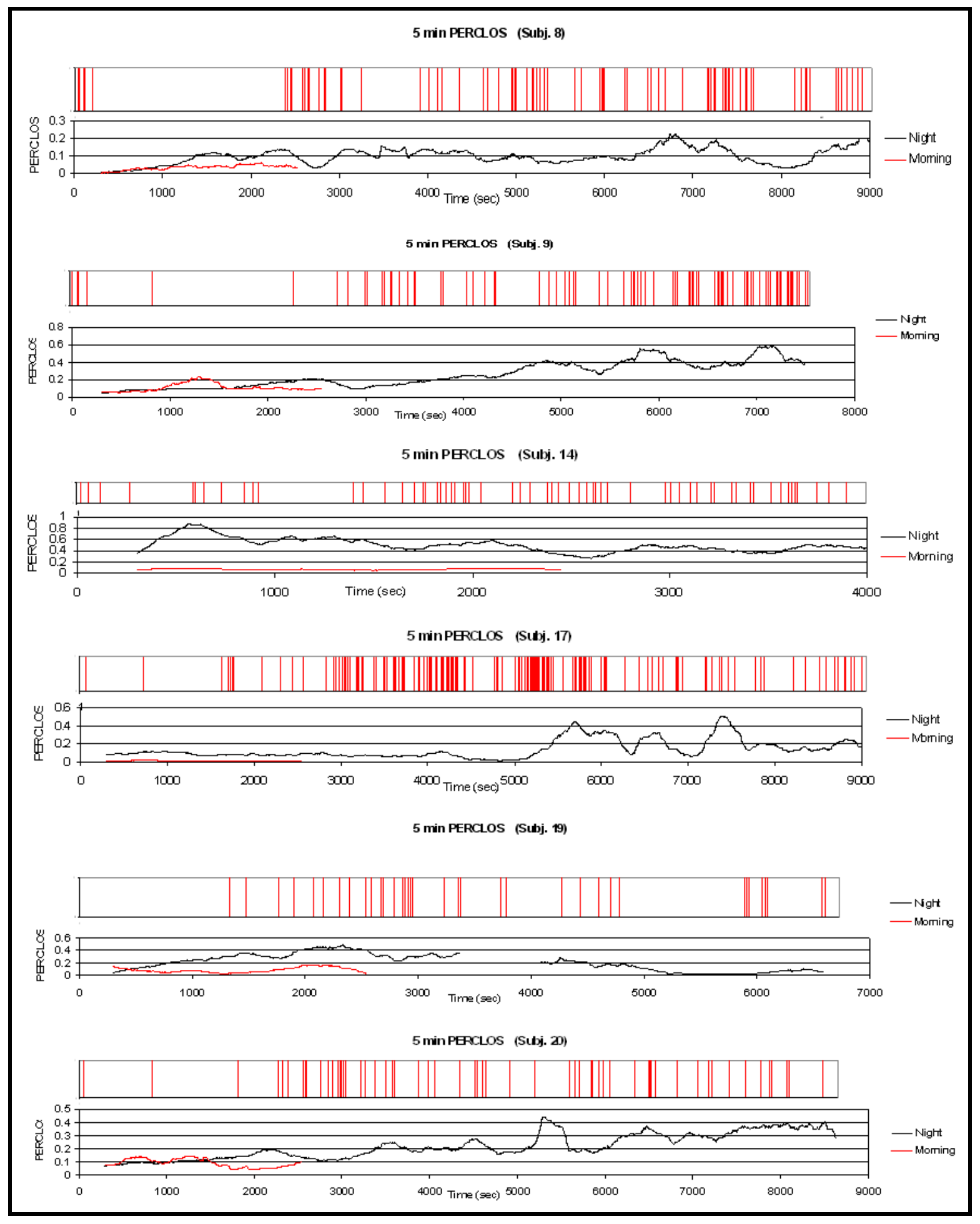

Figure 5.24: Comparison of ANN output with PERCLOS measure 


\section{CRASH PREDICTION RESULTS}

The ability of the drowsy driver detection system to predict crashes is perhaps the most important evaluation criterion. As indicated in the data analysis of the previous chapter, collisions are highly correlated with drowsiness. Thus, warnings issued before a collision can be seen as legitimate warnings as opposed to false alarms. Moreover, the primary purpose of the system is to prevent collisions arising from drowsiness. Thus, the most important assessment metric for a drowsiness detection system is the ability to issue a warning in a timely fashion before an accident is caused by driver fatigue. An analysis of the accidents in the simulation system indicates that this system is able to predict all of the initial crashes that occurred during the experiment.

During the experiment, drivers fell asleep while driving and crashed the vehicle by colliding with other vehicles, departing the roadway, or running into a barrier. In Figures $5.8-5.20$, these crashes are indicated by a black line. There were a total of 130 crashes recorded.

To further investigate the system detection performance during the early stages of drowsiness (i.e., at drowsiness onset), the research team looked at the 5 minutes preceding the two first crashes of each driver. Only the first two crashes were examined, because the driver may remain drowsy through multiple crashes. Thus, they may be little or no steering activity in between sequential later crashes. In reality, the driver would be unlikely to continue driving after experiencing one crash. However, to expand the sample size of the data, the first two crashes were considered.

When the detection system is coupled with a warning system, detailed analysis must be done to evaluate the extent to which fatigued drivers may use the warning system to enable them to continue driving. This concern, as well as other issues that arise with a warning system, is discussed in detail in the next chapter.

An evaluation of the steering-only ANN shows very good performance under the crash prediction metric. The system issued a warning in a timely fashion for $100 \%$ of the first two crashes experienced by any of the subjects. For all of these crashes, the model based on steering data only output at least one warning in the 5 minutes preceding the crash. On average, 5.6 warnings were issued during the 5 minutes preceding the crash. The first warning was issued on average 3 minutes and 56 seconds prior to the crash and the final warning was issued on average 16 seconds before the crash.

\section{SUMMARY AND COMPARISON WITH PREVIOUS DATA}

In conclusion, the performance of the drowsy driver detection systems for truck drivers was acceptable. The performance of the system was similar to previous systems that detected drowsiness in car drivers. The truck driver fatigue detection systems performed extremely well in the most important evaluation metric, timely crash prediction. 
Results of the car simulator study are provided in Appendix A. A comparison of the two studies shows that the results are very similar. The two experimental conditions were not the same and differed in the type of simulator and driver (subject) population, although the amount of sleep deprivation were the same. Table 5.20 shows the results for both of these studies. The percentage accuracy during training decreased slightly for the truck study. The number of false alarms increased slightly for the truck study.

Table 5.20: Performance comparison of truck and car fatigue detection systems

\begin{tabular}{|c|c|c|c|c|}
\hline \multirow{2}{*}{} & \multicolumn{2}{|c|}{ Truck Study } & \multicolumn{2}{c|}{ Car Study } \\
\cline { 2 - 5 } & Steering & Steering+Eye & Steering & Steering+Eye \\
\hline False Alarms & $14 \%$ & $9 \%$ & $13 \%$ & $7 \%$ \\
\hline Accuracy & $85 \%$ & $88 \%$ & $86 \%$ & $92 \%$ \\
\hline $\begin{array}{c}\text { Crash Prediction } \\
\left(1^{\text {st }} \text { crash) }\right.\end{array}$ & $100 \%$ & $100 \%$ & $100 \%$ & $100 \%$ \\
\hline
\end{tabular}

Table 5.20 also shows that the system was able to predict $100 \%$ of the first crashes for all of the subjects. (Note: After the first crash in the simulator, drivers continue driving and subsequent crashes were possible.) Moreover, these collisions were detected in a timely fashion with an average of 5.6 warnings issued in the 5 minutes prior to a collision. The first of these warnings was issued on average, 3 minutes and 56 seconds prior to the crash. Consequently, this detection system holds the promise of safer roadways when coupled with a warning system.

The steering wheel signal was also visually observed to detect any signal degradation prior to the first crash for each subject. Table 5.21 shows the comparison of PERCLOS, steering wheel angle signal observation, and ANN steering detection algorithm results for each subject. The subjects showed signal degradation anywhere from 12 to 1 minute prior to their first crashes. Moreover, ten out of eleven drivers, who experienced crashes, had steady or flatten steering signal (phase 2) phenomenon right before crashes. 
Table 5.21: Summary of Steering Angle Signal Data Observation and ANN Results for the $1^{\text {st }}$ Crash

\begin{tabular}{|c|c|c|c|c|c|c|c|c|c|c|}
\hline \multirow[b]{3}{*}{ Subject } & \multicolumn{8}{|c|}{ Data/Experiment Observation } & \multirow{2}{*}{\multicolumn{2}{|c|}{$\begin{array}{c}\text { ANN Detection Algorithm } \\
\text { Time of detection/Warning before } \\
\text { crash (mm:ss) }\end{array}$}} \\
\hline & \multirow{2}{*}{$\begin{array}{c}\text { First } \\
\text { Crash } \\
\text { Time } \\
\text { (Min:Sec) }\end{array}$} & \multirow{2}{*}{$\begin{array}{c}\text { Comment on } \\
\text { Eye } \\
\text { Any Doze } \\
\text { Off over } 3 \\
\text { min before } \\
\text { crash }\end{array}$} & \multicolumn{3}{|c|}{ PERCLOS } & \multicolumn{3}{|c|}{ Steering } & & \\
\hline & & & $\begin{array}{l}1 \mathrm{~min} \\
\text { before } \\
\text { crash }\end{array}$ & $\begin{array}{l}3 \text { min } \\
\text { before } \\
\text { crash }\end{array}$ & $\begin{array}{l}5 \text { min } \\
\text { before } \\
\text { crash }\end{array}$ & $\begin{array}{c}\text { signal } \\
\text { degradation } \\
\text { detectable by } \\
\text { human } \\
\text { inspection } \\
\end{array}$ & $\begin{array}{c}\text { Steady at } \\
\text { Crash Point }\end{array}$ & $\begin{array}{l}\text { Min before } \\
\text { crash } \\
\text { (Min:Sec) }\end{array}$ & $\begin{array}{l}\text { Farthest } \\
\text { warning } \\
\text { (Min:Sec) }\end{array}$ & $\begin{array}{l}\text { Closest } \\
\text { warning } \\
\text { (Min:Sec) }\end{array}$ \\
\hline 2 & No Crash & & & & & & & & & \\
\hline 7 & $36: 47$ & Yes & $\mathrm{N} / \mathrm{A}$ & $\mathrm{N} / \mathrm{A}$ & $\mathrm{N} / \mathrm{A}$ & Yes & Steady & $7: 15$ & $3: 45$ & $0: 15$ \\
\hline 8 & $148: 36$ & Yes & $20 \%$ & $18 \%$ & $16 \%$ & Yes & Steady & $5: 00$ & $2: 15$ & $0: 15$ \\
\hline 9 & $87: 45$ & Yes & $64 \%$ & $42 \%$ & $41 \%$ & Yes & Steady & $7: 00$ & 8:00 & $0: 45$ \\
\hline 10 & $51: 31$ & Yes & $44 \%$ & $44 \%$ & $43 \%$ & Yes & Steady & 12:00 & $6: 25$ & $0: 30$ \\
\hline 11 & $65: 33$ & Yes & $31 \%$ & $33 \%$ & $35 \%$ & Yes & Steady & $3: 30$ & $3: 30$ & 3:00 \\
\hline 13 & $38: 55$ & Yes & $12 \%$ & $12 \%$ & $12 \%$ & Yes & Steady & 9:00 & $2: 15$ & $2: 45$ \\
\hline 14 & $12: 00$ & Yes & $85 \%$ & $88 \%$ & $86 \%$ & Yes & Steady & $1: 20$ & $1: 00$ & $0: 45$ \\
\hline 15 & No Crash & & & & & & & & & \\
\hline 16 & $137: 44$ & Yes & $6 \%$ & $3 \%$ & $4 \%$ & Yes & Not Steady & $3: 00$ & $3: 45$ & $0: 15$ \\
\hline 17 & $103: 47$ & Yes & $28 \%$ & $40 \%$ & $32 \%$ & Yes & Steady & $10: 00$ & $8: 45$ & $0: 15$ \\
\hline 19 & $37: 20$ & Yes & $33 \%$ & $39 \%$ & $39 \%$ & Yes & Steady & $8: 00$ & $2: 15$ & $2: 15$ \\
\hline 20 & $110: 46$ & Yes & $53 \%$ & $41 \%$ & $37 \%$ & Yes & Steady & $15: 00$ & $2: 30$ & $2: 30$ \\
\hline Average & & & $34 \%$ & $32 \%$ & $31 \%$ & & & $6: 36$ & $4: 03$ & $1: 13$ \\
\hline
\end{tabular}




\section{DRIVER WARNING}

The drowsiness detection system analyzes data for a specific time interval and then classifies that interval as wake or drowsy (in previous experiments the onset of crash was considered a definite drowsy state). However, when and how should a system warn a driver about the hazardous situation is another area of driver/human factors research? To effectively inform the driver of his drowsiness state and prevent him from potentially hazardous driving situations, a driver's assistance system needs to be coupled with the drowsiness detection system and integrated in the vehicle cabin. The design and integration of a warning system is directly related and dependent on the warning algorithm. The timing, intensity, and nature of the warning system to be integrated with the detection algorithm are described. This effort builds upon the existing data and knowledge of FMCSA in this field. This research describes the challenges involved in such a warning system. Specifically, this chapter:

- Explores different aspects of a driver alarm system

- Explores all the available and under development systems

- Defines design guidelines for a driver drowsiness warning system

Although extensive research has already focused on warning systems for collision avoidance, lane departure avoidance, and speed adaptation, warning systems for driver drowsiness mitigation have received less attention. Given the human factors issues and technical challenges involved in this type of warning systems, more research is needed to address the remaining efficiency challenges and potential drivers' acceptance issues.

Different levels of persistence and intervention have been identified for in-vehicle drivers' assistance systems. Persistence can range from voluntary systems, which can be disabled by the driver, to mandatory systems, which are always on. Devices can vary by their level of intervention and include advisory, warning, recording, and automatic control systems. Advisory systems provide information to the driver such as his/her current drowsiness state. Warning systems provide alarms when the system determines that immediate driver action is needed to avoid a potentially hazardous situation. Recording systems record drivers' behavior and driving performance information for review by drivers, fleet operators, vehicle owners, or law enforcement authorities. Systems with automatic control intervention can, for example, use braking forces control to slow the vehicle down and eventually bring it to stop.

For driver drowsiness applications, unless the system knows the vehicle position, orientation, and kinematics in real-time during driving, the system should not take control of the vehicle. A drowsiness assistance system should therefore be either an advisory or warning system, whose role is to redirect drowsy drivers' attention to the driving task, or a recording system for post-driving evaluation of the drivers' fatigue level during their shifts. 


\section{WARNING SYSTEMS CHALLENGES}

The main role of in-vehicle warning systems for driver drowsiness mitigation is to redirect drowsy drivers' attention to the driving task. Because unexpected effects due to a sudden warning alarm can cause undesired drivers' reaction and lead to risky driving situations, the warning system should be capable of breaking driver's drowsiness in an effective manner without startling the driver (Vallet et al, 1993; Haworth and Vulcan, 1991).

In the interaction between the driver and the automated warning system, both parties are capable of error. A warning system should be able to generate response for optimum system effectiveness and driver interaction. The following considerations need to be adequately addressed if the system is to achieve effective safety improvements: system effectiveness, user acceptance and trust, issuance of false alarms, training issues, and issues of warning understanding.

\section{System Effectiveness}

Various measures related to the driver's drowsiness could be used to evaluate the effectiveness of a warning system. A comparison of driving performance (e.g., the number of crashes or lane departures avoided, standard deviation of the vehicle lateral position, etc.) and driver's physical and physiological variables (e.g., driver's eye activity, EEG, heart rate, etc.) with and without the warning system can help evaluate the warning system effectiveness for driver's drowsiness mitigation. However, accurate evaluation of a driver drowsiness warning system would require testing and comparing such variables under identical levels of driver drowsiness. Since a perfect measure for drowsiness is not available, some variability during the testing of the system efficiency may be introduced.

\section{User Acceptance and Trust}

Previous research has identified driver's trust in automated in-vehicle warning systems as a critical factor determining system effectiveness. User interaction with the system is, therefore, highly determined by his level of trust in the system (Itoh et al, 1999; Lee et al, 1998; Lee and Moray 1992; Muir 1994).

Driver reactions to a warning system can vary significantly for different drivers. An ideal drowsiness warning system should be able to take individual differences into account when generating a signal. This adaptability of the system to drivers' reaction and preferences can, for example, be envisaged by graded levels in the warning signal.

Issues of drivers ignoring warnings have also been reported in the literature. Uaneras et al (2005) indicated that one third of subjects responding to alarms from a back-up warning system heard the warning, but decided to ignore it because they did not perceive any imminent danger. 
User acceptance can be determined both by subjective questionnaires addressed to the drivers or by monitoring drivers' use of the system. Drivers, who reject or are annoyed by the system, may ignore the system warning signals or simply switch the system off.

\section{Issuance of False Alarms}

The initial consideration for setting the decision threshold of an automated warning system is the cost of a false negative (missed alarms) versus that of a false positive alarm (Parasuraman et al, 1997).

\section{False positives}

False alarms can lead to users' decreased trust in the system and delayed response. Gupta et al (2002) showed that drivers trust low sensitivity warning systems more than high sensitivity warning systems.

Enriquez and MacLean (2004) showed that false positives alarms have a deleterious effect on driver's ability to interact effectively with a haptic warning system. Excessive number of false positives affects driver's trust in the system. False negatives (missed alarms) showed no influence on driver's ability to use the warning signal. Bliss and Acton (2003) also observed that alarm reliability level significantly affects drivers' reaction to a collision warning system.

Other studies have shown that the number of false alarms is not necessarily detrimental to the system/driver performance up to some point. Dingus et al (1997) observed that distrust in a collision warning system due to an excessive number of false alarms occurs when a $60 \%$ rate of false alarm is reached. Although they mentioned that drivers were somewhat affected by the false alarms, Ben-Yaacov et al (2002) found similar results. Both concluded that the warning system does not necessarily need to be perfect for the driver to adopt safer driving strategies.

CISR believes that an excessive number of false alarms are not as problematic for drowsiness warning system as for other types of driver's assistance systems. Where a false collision warning alarm can lead to a driver's sudden braking and create a hazardous driving situation, a false drowsiness warning would not trigger any inappropriate reaction from an alert driver. However, if drowsiness false alarms are not safety critical, they can

probably contribute to driver's annoyance if they are too frequent and may lead the driver to switch the system off.

\section{False negatives}

Missed alarms in the driver assistance system - like a drowsy driver warning system can lead to hazardous driving situations and most probably to crash. It is, therefore, safety critical that the number of missed alarms nears zero. A compromise between the number of false positive and false negative alarms needs to be found when assessing the warning system sensitivity. 


\section{Training Issues}

Proper training for a warning method can help drivers to better react to the warning signals. Adequate mental processing of a warning can be significantly improved by training. Drivers' decisions and actions following a warning signal can be sped up and automated if they have previously learned how to handle the signal information.

The benefits gained from training may depend on individual differences. For example, working memory capacity for memorizing warning icons has been shown to differ significantly between drivers of different age groups (Lesch, 2003). Although this study only investigated subjects' capacity to understand and memorize visual warning symbols, if such results should prove to be extendable to other types of warning signals, the issue of individual driver differences in analyzing a warning signal should be considered when designing a warning system for driver drowsiness.

\section{Issues of Warning Understanding}

Ease of warning meaning recognition is also important (Suzuki and Jansson, 2003). Drivers' mental model for responding to the warning stimulus should effectively interpret the real conditions of the vehicle. If this is achieved, the drivers will respond in a correct manner to the warning. Otherwise, drivers can misunderstand the warning and adopt an even riskier behavior. Meredith and Edworthy (1995) showed that ambiguous meaning of alerts could undermine systems performance and increase workload.

\section{WARNING STRATEGIES}

Various strategies can be envisaged for alerting the driver of his/her drowsiness state. The following questions should guide the design of an effective drowsiness warning system:

- What information to provide to the drowsy driver (i.e., nature and content of the warning message)?

- When to provide this information to the driver (i.e., timing and sequence of the alert)?

- How to convey this information to the driver (i.e., modality of the warning signal)?

\section{Warning Content}

A warning must attract the driver's attention, convey the correct message, and suggest the corrective action to be taken. (Bekiaris and Nikolaou, 2004)

A driver drowsiness warning system should be able to present accurate information to the driver about his/her drowsiness level. Excessive discrepancies between the driver's understanding of the alarm and the actual driving situations may impair the system effectiveness. In his urgency mapping principle, Edworthy (1994) advocates that the urgency of the situation should match the perceived urgency of the alerts. 
A simple drowsiness warning system should be limited to informing the driver about his/her status. More advanced systems that include situation awareness capabilities can possibly advise the driver of the appropriate corrective action to take given the current driving conditions. Such driver support systems would consider the vehicle kinematics parameters and surrounding environment to work in a situation-specific manner (Onken, 1994).

Ideally, drivers should not use the warning system as a way to drive longer when fatigued, but should understand from the message that they must bring the vehicle to a stop to get some rest. The warning content should be clear enough that no driver misinterpretation is possible, but should also be kept simple to avoid excessive attention from drivers.

\section{Type of Warning}

Driver sensitivity to warning signals can be very different. Some drivers may understand a warning immediately and, therefore, be very quickly annoyed by repeated warnings, while other drivers may need to be warned several times before they respond.

Graded warning, as compared with single-stage warning, can be a simple way to adapt warning to individual differences. A system able to account for the drivers' response to a warning would be suitable for all types of drivers. Such a system would stop alerting highly sensitive drivers early because they already responded to the warning, while continuing to alert less sensitive drivers until they respond to the warning. Horowitz et al (1992) also underlined the importance of warning adaptability to individual behaviors. However, the authors believe that the adjustment of the warning frequency by the driver himself, needs further analysis as drivers may not necessarily be able to objectively determine the warning setting that can optimize their interaction with the system.

Graded warning was also shown to improve driver's understanding of warning and was preferred by drivers over single warning, at least for visual displays (McGehee et al, 1994). Gupta et al (2002) reported that graded warnings of an imminent driving hazard based on urgency of the situation resulted in improved drivers' vehicle control.

Bekiaris and Nikolaou (2004) recommend two levels of warning for cautionary and imminent cases. Cautionary warning should be generated in the case of a hazardous driving condition requiring no immediate action from the driver. Imminent warning should be triggered when immediate response from the driver is necessary to avoid a crash. In a comprehensive literature overview of in-vehicle warning systems, NHTSA recommends variable-intensity warning displays for driver drowsiness warning (COMSIS, 1993a, b).

\section{Warning Timing}

The determination of the alarm timing is crucial for the alarm effectiveness (Janssen and Nilsson, 1993). A poorly timed warning may undermine driver safety (McGehee et al, 2002). An alert issued too early may be ignored by drivers if they are unable to perceive 
the cause of warning, and an alert issued too late may be ineffective or disrupt the ongoing driver's action (Lee et al, 2002).

Drivers may judge whether an alarm is trustworthy or not according to its timing independent of its validity (Abe and Richardson, 2004). Early timing can be associated with nuisance and false alarms. This can influence driver perception of alarm validity and reliability with consequent effects on system acceptance and effectiveness.

If a warning is issued early or just after driver's response has been initiated, it acts to speed up the driver's response. However, if the alert signal is issued during the driver's response planning or when he/she is in the middle of his response maneuver, it may interfere with the response decision or execution and can be viewed as unhelpful and annoying (Brown et al, 2001, McGehee et al, 2002). Late alarms can also lead to decreased drivers' trust in the warning and, therefore, degrade warning effectiveness.

Alarm timing, like the type of warning, ideally would adapt to individual drivers. For example, when defining an alarm's timing, drivers' behavior inputs (i.e., reaction time) should ideally be taken into account (Kiefer, 2000).

\section{Warning Modality}

Independent of the warning mode used, the warning signal should be capable of overcoming sleep inertia but should not induce a startled reaction of the driver (Tepas and Paley, 1992). Various modes of warning have already been studied for in-vehicle driver's assistance systems like collision and lane departure avoidance or speed adaptation systems. All methods of warning have been proven to be effective to some degree depending on their intended application. However, an objective evaluation and comparative effectiveness of these solutions for driver drowsiness warning is not yet available.

Existing warning solutions can be classified in the following categories: visual warnings, auditory warnings, and haptic warnings. For multi-modal warning systems, the warning sequence must also be taken into consideration.

\section{Visual Warnings}

Visual information can be displayed by in-vehicle monitors, head-up-displays, icons on instrument panel and rear and side view mirrors, etc. Various intensities, colors, and warning messages and signs can be envisaged. However, visual display is not an appropriate warning mode to alert a drowsy driver, who may sleep through the warning. In addition, since driving is primarily a visual based effort, visual displays may draw the driver's attention away from the driving task more than other warning modes.

However, visual displays have proven effectiveness in providing the driver with detailed information about the driving situation (e.g., headway distance, time-to-collision, vehicle lateral position in lane, etc). This type of warning could be used as a secondary warning to help the driver take the adequate driving actions, once he/she has been alerted by a primary warning. 


\section{Auditory Warnings}

Tone and voice/speech messages can be used to convey information to the drivers about an imminent driving hazard. Tones, beeps, rumble strip sounds, etc. were shown to be effective in attracting drivers' attention and enabling them to quickly respond to critical situations, where immediate response is needed (Hirst and Graham, 1997; Ross et al, 1996; Horowitz et al, 1992). However, they can not convey detailed information to drivers about the hazardous driving situation.

Different intensities of acoustic stimulus may be needed to arouse drivers from sleep. An ideal drowsiness warning system should accommodate these differences either by proper calibration to each individual driver before using the system or through a learning capability that automatically adjusts to each driver's sensitivity to alarms. Bekiaris and Nikolaou (2004) highlighted the need for a drowsiness warning system to be adjustable in presence, intensity, and type of warning to individual drivers' preferences. Green et al (1995) recommends that an auditory warning be 15 decibels above the masked threshold of the vehicle cabin sound environment. A maximum absolute intensity of $115 \mathrm{db}$ was suggested as intense sounds can induce drivers' annoyance and workload. Ross et al (1996) suggested that no more than three tones be generated inside the vehicle to avoid driver confusion.

Speech and voice messages can be used as secondary warnings to provide alerted drivers with more insight about the driving conditions and actions to be taken. Speech messages should be short and their meaning obvious to avoid excessive attentional demand. Fahey et al (1995) found that a speaking rate of 150 words/minute was appropriate for speech messages.

Lloyd et al (1999) identified the advantages and disadvantages of different tone and voice warning modes, as shown in Table 6.1.

Table 6.1: Tone and voice warning messages from Lloyd et al (1999)

\begin{tabular}{|c|c|}
\hline \multicolumn{2}{|c|}{ Warning Tone } \\
\hline $\begin{array}{l}\text { - Omni-directional; orienting stimulus } \\
\text { - Under normal conditions, demand less } \\
\text { - } \quad \text { Prtention than voice } \\
\text { - } a l, 1994 \text { ) } \\
\text { - } \text { Language independent } \\
\text { Auditory icons which match a driver's mental } \\
\text { responses (Graham and Mitchell, 1994) }\end{array}$ & $\begin{array}{l}\text { Difficult to accommodate } \\
\text { hearing-impaired drivers } \\
\text { - Unable to convey detailed } \\
\text { information } \\
\text { - Signal detection problem under } \\
\text { high ambient noise conditions } \\
\text { - Could cause unwanted startled } \\
\text { responses } \\
\text { - Integration with other in- } \\
\text { vehicle devices could lead to } \\
\text { warning cacophony }\end{array}$ \\
\hline
\end{tabular}




\begin{tabular}{|l|l|}
\hline \multicolumn{2}{|c|}{ Voice Warning } \\
\hline - Omni-directional; orienting stimulus & - Difficult to accommodate hearing- \\
- Processed faster than visual stimuli & impaired drivers \\
- Language dependent \\
- Able to convey detailed information & - Signal detection problem under high \\
- Speech may be more effective in high & ambient noise conditions \\
stress situations because speech meaning & - Could cause unwanted startled \\
is over-learned (Edman, 1982) & responses \\
& - Annoying if unnecessary \\
& - Integration with other in-vehicle devices \\
& could distract the driver with abundance \\
& of verbal messages \\
& - May demand more attention than tone \\
\hline
\end{tabular}

Lee and Patterson (1993) indicated that spatial auditory warnings can help drivers locate an imminent danger and, consequently, enhance their corrective response. Spatialized sound alerts have also been shown to speed up a driver's reaction to an imminent driving hazard, (Hoshino et al, 2002). However, the effectiveness of specialized sound warnings for drowsy drivers has not yet been evaluated.

\section{Haptic Warnings}

Existing literature indicated that haptic displays produce less mental workload on the driver and possibly shorter reaction times. Vibrations in the driver's seat, seat belt (Bekiaris and Nikolaou 2004), steering column (Naab and Reichart, 1994; Isomoto et al, 1995), throttle pedal (Janssen et al, 1994), or brake pedal (Lloyd et al, 1999) can be used to alert the driver of an imminent driving hazard. Previous research has consistently reported the potential for haptic feedback to provide information to drivers that enhances performance (Payandeh et al, 2002; Steele et al, 2001).

Lerner et al (1996) found high drivers' acceptance levels and high effectiveness rates of a safety belt vibration system for drowsy driver warnings. A vibration frequency between 100 and $300 \mathrm{~Hz}$ was found to be adequate for optimum driver's response.

Lloyd et al (1999) mentioned that haptic cues present many advantages as they are quickly perceived by drivers - hence, reducing their reaction time - and can be used to cause an oriented response of the drivers if the stimulus is sufficiently intense. To achieve proper and fast reaction of the drivers to the haptic warning, Lloyd states that the warning should be transmitted through the same device (i.e., pedal, steering wheel) that drivers would use to respond to the hazardous situation.

Although they found that warning modality had little effect on driver performance, Lee et al (2004) reported that haptic warnings were preferred by drivers to auditory warnings on several dimensions including trust, overall benefit to driving, and annoyance. 
The main disadvantage of haptic warnings is that they are unable to convey detailed information to drivers. Drivers alerted by a haptic warning could benefit from a secondary warning (e.g., speech message) to better inform them of the current driving situations and to advise them of the correct actions to take.

\section{Warning Sequence}

Depending on the goal of the display, the optimum warning modality can be different. Previous research agreed on the safety potential of generating two stages of alert for drowsy driver warning. Auditory and haptic displays are probably more advantageous in imminent warning situations in which the driver is drowsy. This type of warning will redirect his attention to the driving task. A second stage of warning can then be generated to inform the driver that the alarm relates to his drowsiness state or provide $\mathrm{him} /$ her with information on current driving conditions. Speech messages could be used for this purpose (Bekiaris and Nikolaou, 2004).

Bekiaris and Nikolaou also underlined the need for maintaining drivers' alertness after warning. Various perspectives can be envisaged here:

- The warning system could be supplemented by a driver's stimulating system (e.g., providing the driver with blasts of cold air, release of various scents, for example peppermint, smell of coffee, etc.).

- The warning should be clear enough that the driver understands that he/she should pull over and get some rest. A system that would allow drivers to drive longer in advanced fatigue states would create a higher volume of drowsiness occurrences and, therefore, fail in meeting any safety enhancing objectives.

- The warning system could communicate with the outside world and send a signal to the monitoring center of a commercial fleet, or call a preset phone number or the police, or flash lights and blow horn to inform the others of the danger.

\section{REMAINING ISSUES AND RESEARCH OPPORTUNITIES}

\section{Driver Behavioral Adaptations}

The effect of drivers' behavioral adaptations after extended exposure to the system should also be considered before a drowsiness warning system is integrated in a real vehicle. Adverse effects can potentially be experienced including:

- Drivers rely too much on the system and drive in unusually advanced fatigue states thinking that the system would alert them if necessary

- Drivers get used to the system and pay less attention to the warnings

- Drivers persevere in driving after a warning and are susceptible to get drowsy again (Haworth and Vulcan, 1991) 
Previous studies on vigilance and information acquisition capability proved that subjects' acquisition rates can decline significantly after few minutes on task. Information detection can fluctuate over time independently of subjects' alertness/drowsiness level (Makeig and Inlow, 1993).

\section{Public Attitudes}

Other issues will also have to be addressed before wide commercial availability of a drowsiness warning system including public attitudes towards the system: willingness to pay for a drowsiness warning system, levels of effective use of the system in the case that drivers have the option of switching the system off, etc.

\section{Interaction with Other Safety Systems}

As other in-vehicle driver's assistance systems are also being developed and will soon be available in real vehicles, potential issues of interference between different systems will need to be analyzed. For example, in the case of a drowsy driver drifting out of his traveling lane, alerts from both a lane departure warning system and a drowsiness warning system could be triggered simultaneously. This could cause a driver's misunderstanding or confusion and prevent him from taking the appropriate driving action. Finally, an assessment of effective reduction of crashes related to drivers' drowsiness would be needed to objectively evaluate the system effectiveness.

\section{Evaluation of a Driver Drowsiness Warning System in a Driving Simulator}

Experimental tests should be conducted before a driver drowsiness warning system can be widely deployed. The CISR truck driving simulator could be used to evaluate the benefits of a drowsiness warning system: overall system effectiveness, driver acceptance, driver behavioral adaptation to the developed system, evolution of driver's driving and resting strategies and other safety enhancing effects, etc. Subjective and objective evaluation techniques could be used to study the performance of a new system. Comparative evaluation of various warning systems for drowsiness mitigation could also be envisaged. 


\section{CONCLUSIONS AND FUTURE WORK}

This report described the successful tests of a truck driver drowsiness detection system. The hypothesis underlying this system is that fatigued drivers exhibit different steering patterns than non-drowsy drivers. Previous tests of this system demonstrated the effectiveness of this system for car drivers. Differences in car and truck driving, including the professional experience of truck drivers, the different dynamics of trucks, and the different feel of steering were amply apparent in the setup of a truck driving simulator laboratory. However, this project demonstrated that these differences have a minimal impact on the performance of the drowsy driver detection system.

After conducting a literature review of relevant studies in fatigue, drowsy driver detection systems, and vehicle-based warning systems, a truck driving simulator was constructed for the experiments that assessed the effectiveness of the drowsy driver detection system. The fixed base simulator included a 135 degree field of view forward projection system; projection systems for both side mirrors; a realistic sound system; realistic feels for the brake, acceleration, and clutch pedals; a gearbox; and a complete data recording system including full range of control inputs using optical encoders, vehicle kinematics, eye monitoring, and video recording from multiple camera angles. The simulator was validated by both truck drivers and experts in truck simulators.

Once the simulator had been constructed and validated, the drowsy driver experiment was conducted. Fourteen subjects, who were truck drivers with valid commercial drivers licenses, participated in the experiment which consisted of three driving sessions for each subject. First, the subjects participated in a practice session, which lasted approximately one hour, in which they became accustomed to driving the simulator. Sometime later, usually the next day, the subject would then participate in a one-hour morning session, which was held between 8:30 and 9:30 am. The purpose of the morning session was to establish a baseline of predominantly non-drowsy steering behavior for each subject. During the day, the subject was instructed to avoid napping and excessive caffeine consumption. That night, a member of the research team would drive the subject from their abode to the simulator. The subject would then participate in night driving session starting around 1:30 am, with each session lasting for approximately 2 hours.

The data generated from these experiments was analyzed thoroughly to evaluate inputs for a drowsy driver detection system and performance metrics for the system. This analysis showed a correlation between high amplitude steering corrections and drowsiness. This analysis also indicated that the crashes are strongly correlated with drowsiness. Due to this correlation and the primary purpose of the drowsy driver warning systems, collision avoidance, the ability of the drowsiness detection system to detect drowsiness in a timely fashion prior to a collision was established as the most important evaluation criterion.

Although there was a strong correlation between drowsiness and steering activity, the relationship between these two variables is complex and non-linear, and varies from driver to driver. This complexity and variability precluded the development of a precise model to detect driver drowsiness based on steering activity. On the other hand, 
Artificial Neural Networks (ANNs), with their ability to learn these types of relationships, are a natural approach for this type of system.

Central to the success of an ANN is a data preprocessing stage in which the data are transformed to maximize the ability of the ANN to learn the desired relationship. The drowsy driver detection system developed in this project uses a unique data preprocessing step that discretizes the steering activity in fifteen-second intervals. This discretization enables the ANN to distinguish between low amplitude steering corrections in nondrowsy driving and the larger amplitude steering corrections that characterize drowsy driving.

The performance of both the steering-only and steering-with-eye-tracking ANNs was similar to previous systems that detected drowsiness in car drivers, despite the differences in the experimental conditions for the two studies. These differences included the amount of sleep deprivation, the type of simulator, and the driver population.

The effectiveness of the drowsy driver detection algorithm provides evidence that it would be an important component of a drowsy driver prevention system. This system would incorporate countermeasures, for example, warning systems that minimize the severity of crashes and reduce injuries and fatalities in highway crashes involving trucks. This report presented design guidelines for such a warning system. The timing, intensity, and nature of the warning system to be integrated with the detection algorithm were discussed.

This project lays the foundation for future work in the development and deployment of a drowsy driver detection system. The primary areas for future work are the development and testing of prototype detection systems and the integration of a warning system into the drowsy driver detection system.

Given the success of the drowsy driver detection system in the simulator experiments, the next step in the evaluation of the system is the development of a prototype system that can be deployed in an actual vehicle. The prototype system will include both sensors and a computing system. Since the system relies only on the steering signal for input, the sensor requirements are minimal. This prototype system would then be used to evaluate the system effectiveness, first in track tests, and then in field operational tests. The purpose of these tests is to evaluate the effectiveness of the detection system, rather than a combination of a detection and warning system.

Central to the success of the drowsy driver detection system is the preprocessing step that discretizes the steering signal. Since this algorithm has proven relatively successful on three sets of simulation data with three different steering sensitivities, it is likely that this preprocessing step will enable the detection system to perform well in real word tests. Currently, the ranges in the discretization step are determined offline. When creating the prototype system, one future challenge will be implementing the algorithm online so that the ranges for the discretization are determined automatically.

Based on the design guidelines developed in Chapter 6, the warning interface for the drowsy driver detection system should be developed. Simulator tests should be 
conducted to evaluate the most effective warning modality, timing sequence, and warning level for safely and effectively warning drowsy drivers. After these initial simulatorbased tests, track tests and field operational tests would then be needed to fully evaluate the warning systems. 


\section{APPENDIX A. RESULTS FROM PREVIOUS ANN MODELS}

CISR conducted driving simulator experiments using drivers under sleep deprived conditions to collect data. This data was used to develop models for detecting drowsy driving. Results of this study are presented here. The data preprocessing technique and the training and testing parameters were the same as those described in Chapter 4.

\section{EXPERIMENTAL PROTOCOL}

The experiment was conducted in the CISR car driving simulator laboratory. Twelve subjects with age ranging from 22 to 42 years participated in the study. Most were recruited from the GWU student population. During the experiment, subjects drove the car simulator in a rural highway driving scenario two times. Table A.1 shows the schedule and amount of sleep deprivation for the two sessions.

Table A.1: Amount of Sleep Deprivation

\begin{tabular}{|c|c|c|}
\hline & Session I & Session II \\
\hline $\begin{array}{c}\text { Amount of Continuous } \\
\text { Wakefulness }\end{array}$ & $1-2$ hours & $18-21$ hours \\
\hline Time Schedule & $8: 30 \mathrm{am}-10 \mathrm{am}$ & $1: 00 \mathrm{am}-3: 00 \mathrm{am}$ \\
\hline
\end{tabular}

Data colleted during the experiment was preprocessed and used to train an Artificial Neural Network for detecting steering behavior representing drowsy driving. Data processing techniques are the same as those described in Chapter 4 of this report.

\section{ANN MODELS}

Two ANN models were developed. Each was trained and tested using different type of data - a steering only model and a steering plus eye model. Training, testing, and results of these models are discussed here.

\section{ANN TRAINING (STEERING)}

The shortest experimental session for which data was recorded was 20 minutes (i.e., eighty 15 second time intervals). To balance the data, only the first 80 intervals from the morning session and the last 80 intervals from the night session were used. Data from 10 subjects, comprising a total of 1,600 driving intervals, was randomized and then divided into three separate files: 
- Training file, which includes a set of 750 input-output exemplar vectors

- Cross validation file, which includes a set of 100 input-output exemplar vectors

- Testing file, which includes a set of 750 input-output exemplar vectors

These files contain data in the form of input vectors $\boldsymbol{X}(\boldsymbol{n})$ and output vectors $\boldsymbol{D}(\boldsymbol{n})$, each representing 15 seconds of driving. The ANN performance during training is shown in Figure A.1. The smooth drop in MSE shows that the ANN performed very well.

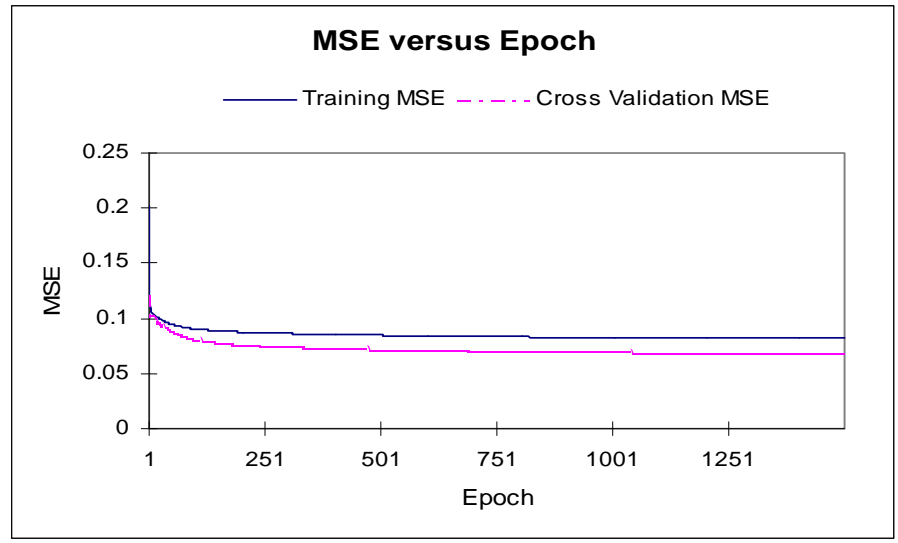

Figure A.1: ANN training performance

\section{ANN TESTING (STEERING)}

Table A. 2 shows ANN performance during testing. The ANN correctly identified 318 out of a total of 371 drowsy intervals ( $86 \%$ accuracy) while 53 intervals were misclassified. The ANN also correctly identified 332 wake intervals out of a total of 379 wake intervals ( $88 \%$ accuracy).

Table A.2: ANN test results (steering)

\begin{tabular}{|c|c|c|}
\hline \multirow{2}{*}{ OUTPUT } & \multicolumn{2}{|c|}{ DESIRED } \\
\cline { 2 - 3 } & WAKE & DROWSY \\
\hline WAKE & 332 & 53 \\
\hline DROWSY & 47 & 318 \\
\hline \% CORRECT & 88 & 86 \\
\hline
\end{tabular}




\section{CRASH PREDICTION}

During the experiment, drivers fell asleep while driving and crashed the vehicle by colliding with another vehicle or with a barrier. The network identified sleep patterns before the crash in all the cases (i.e., an accuracy of 100 percent). The first bar graph in Figures A.2 - A.5 is the ANN (steering angle only) output for all subjects. It can be seen that the network predicted drowsy driving before the crash occurred in all the cases.

\section{TESTING ALL SUBJECTS}

Figures A.2 - A.5 show ANN output of CISR data for all the subjects.

- The first bar graph is output of the ANN based on the steering-only data model.

- The second bar graph shows driver eye activity. Each bar represents the fraction of time when the eye was closed in that particular 15 second time interval.

- The third bar graph is the output of the ANN, using both eye and steering angle data.

Figures A.2 - A.5 show the ANN output, for both morning and night driving sessions, of each subject. Observation of these figures shows that in the majority of cases, there are very few drowsy patterns during the morning driving sessions. During the night driving sessions, when the drivers were highly sleep deprived, there are a large number of drowsy patterns, indicating driver sleepiness. On the average, the ANN classified 9 percent of the total morning driving intervals as drowsy while for the night driving this increased to 50 percent. Table A. 3 shows these percentages for individual drivers.

Table A.3: Percentage of drowsy intervals identified by ANN

\begin{tabular}{|l|c|c|c|c|c|c|c|c|c|c|c|}
\hline \multirow{2}{*}{$\begin{array}{l}\text { Driving } \\
\text { Session }\end{array}$} & \multicolumn{10}{|c|}{ Subject Number } \\
\cline { 2 - 13 } & 1 & 2 & 3 & 4 & 5 & 6 & 7 & 8 & 9 & 10 & 11 \\
\hline Morning & $7.62 \%$ & $6.5 \%$ & $13.8 \%$ & $12.7 \%$ & $10.5 \%$ & $9.76 \%$ & $13.0 \%$ & $3.2 \%$ & $13.8 \%$ & $13.8 \%$ & $4.0 \%$ \\
\hline Night & $36.7 \%$ & $48.2 \%$ & N/A & $37.5 \%$ & $50.2 \%$ & $50.8 \%$ & $62.4 \%$ & $61.4 \%$ & $56.8 \%$ & $54.0 \%$ & $43.4 \%$ \\
\hline
\end{tabular}



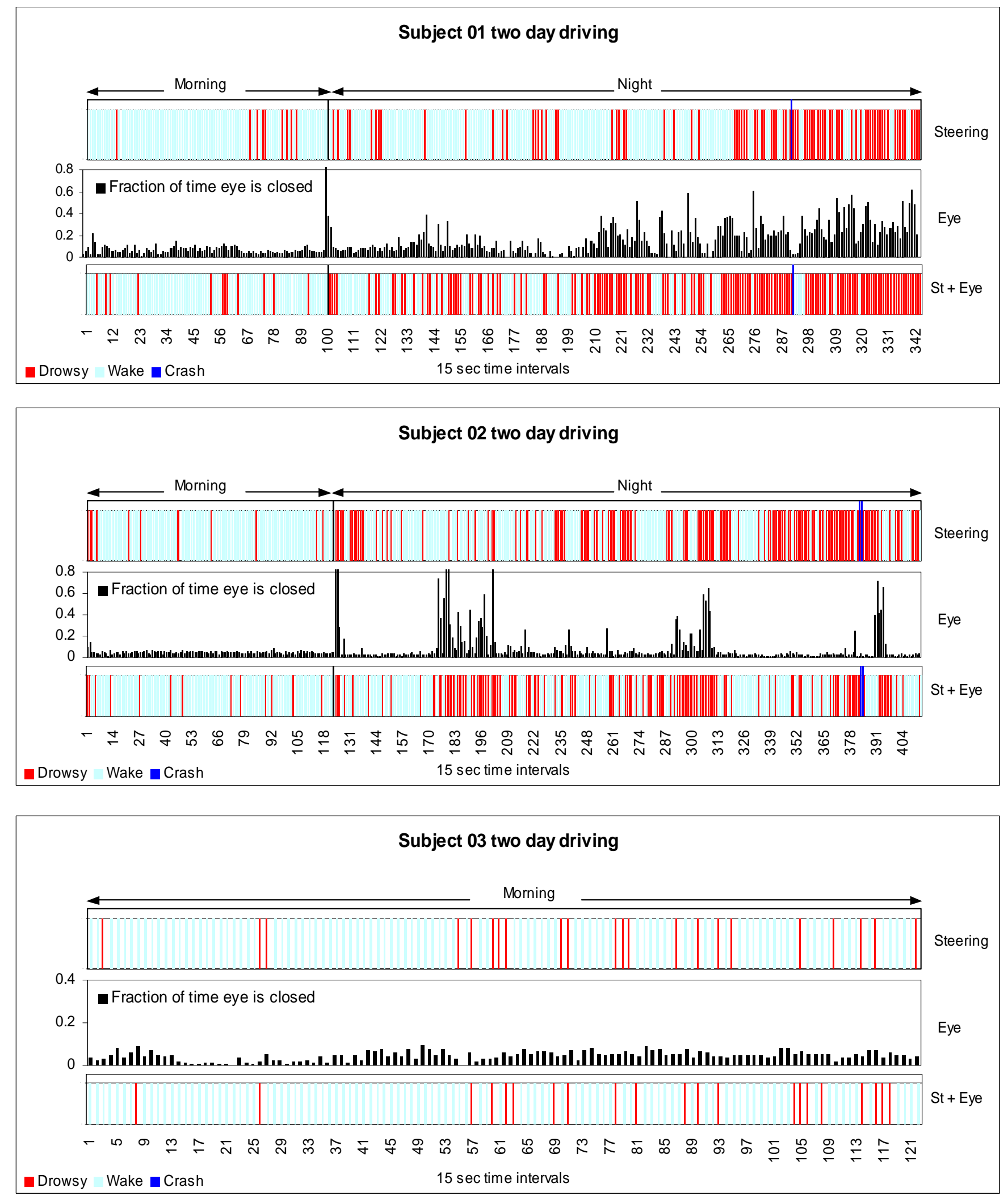

Figure A.2: ANN output for subjects 1 to 3 

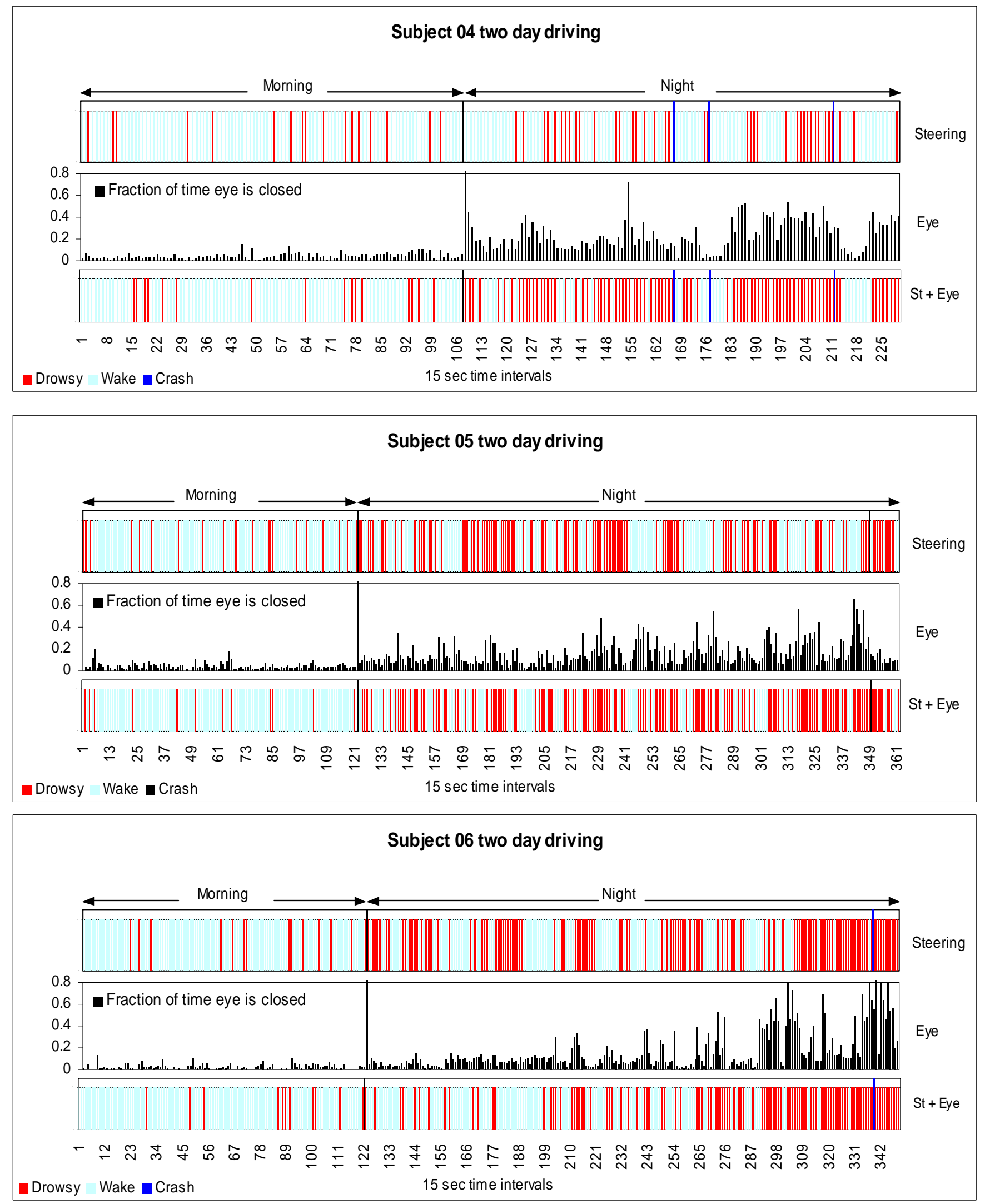

Figure A.3: ANN output for subjects 4 to 6 

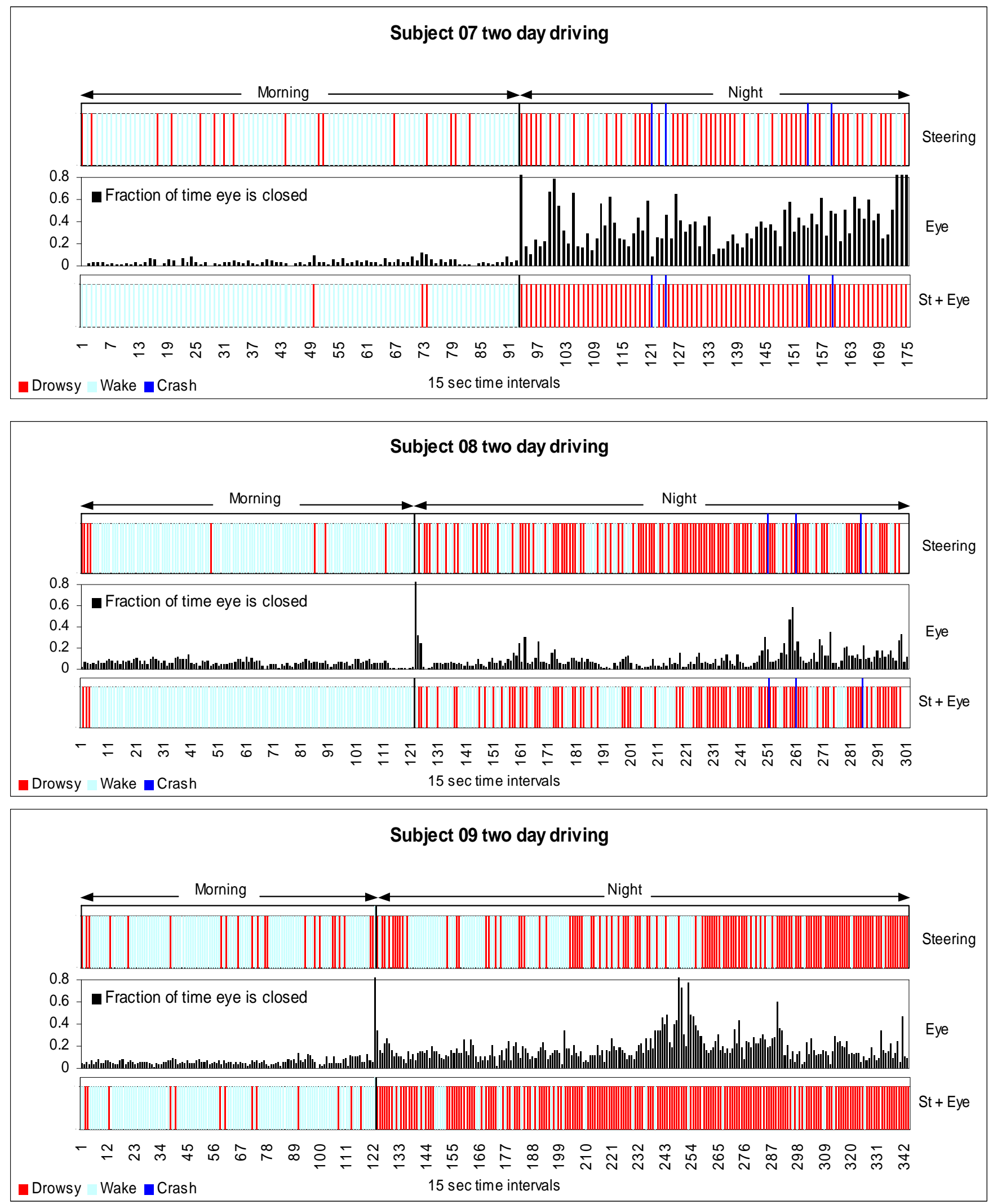

Figure A.4: ANN output for subjects 7 to 9 


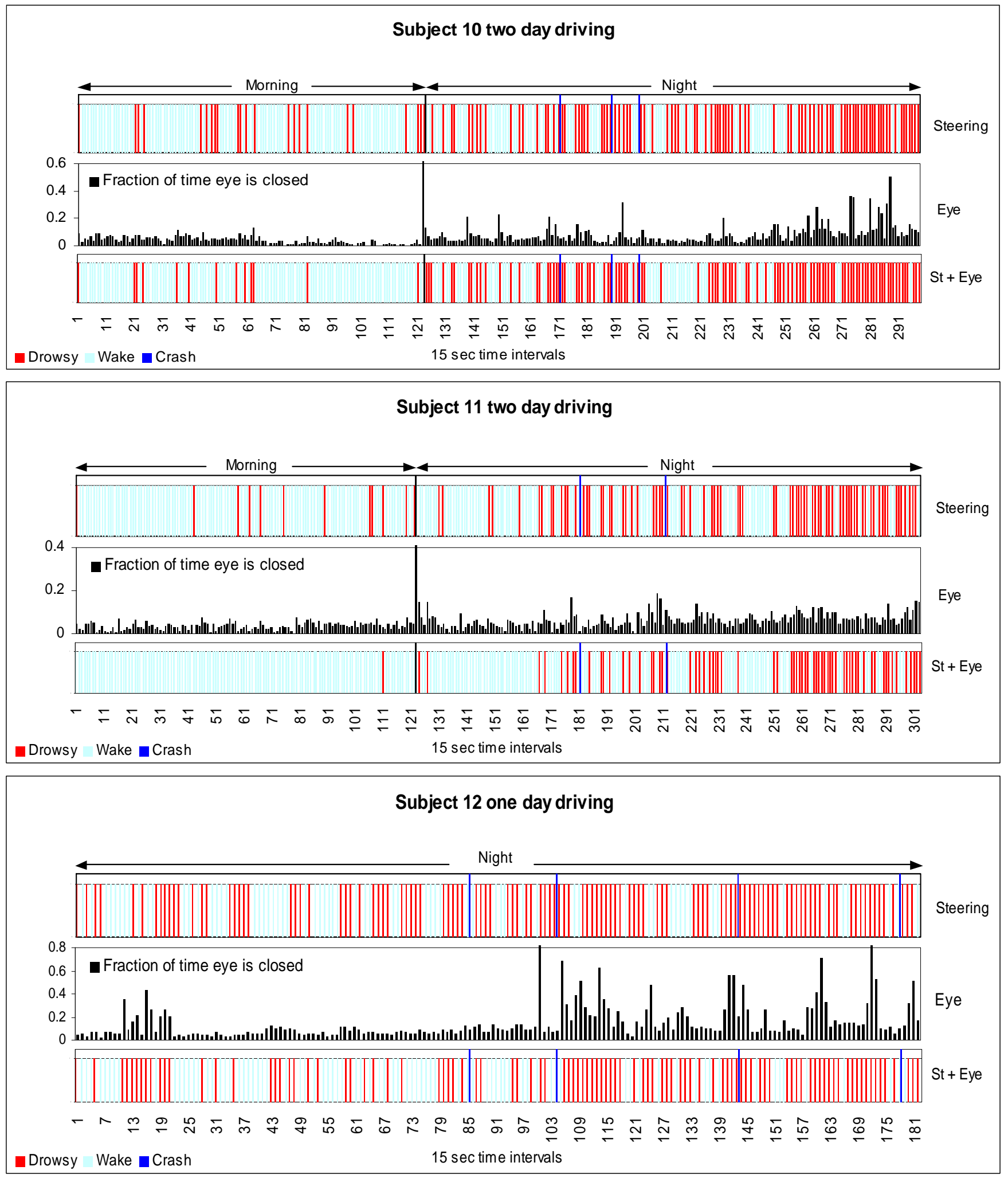

Figure A.5: ANN output for subjects 10 to 12 


\section{COMPARISON WITH PERCLOS MODEL}

PERCLOS is the percentage of time the eye is more then $80 \%$ closed. Although PERCLOS is one of the widely accepted measures in the scientific community for drowsiness detection, it has the following limitations:

- PERCLOS predictions are good only when using large time intervals

- PERCLOS does not take into account variability in eye blinking behavior between individuals

- PERCLOS measures usually require obtrusive in-vehicle equipment

- An eye tracker system can present reliability and measuring robustness problems (see section on Eye Closure Data in Chapter 4)

Although a direct interval-by-interval comparison is not possible due to these limitations, a comparison with PERCLOS data calculated over 6-minute intervals shows good correlation. In more than $80 \%$ of the PERCLOS six minute drowsy intervals, the ANN output shows one or more 15 second drowsy intervals. Figure A.6 shows the PERCLOS and ANN output for three drivers.

\section{COMBINED ARTIFICIAL NEURAL NETWORK MODEL}

This ANN model has was trained on data from both eyes and steering. The design and architecture of the ANN was similar to the one explained in Chapter 4 with the following architecture:

- The input layer has 12 neurons corresponding to the input vector $\boldsymbol{X}(\boldsymbol{n})$

- The hidden layer has 27 neurons 

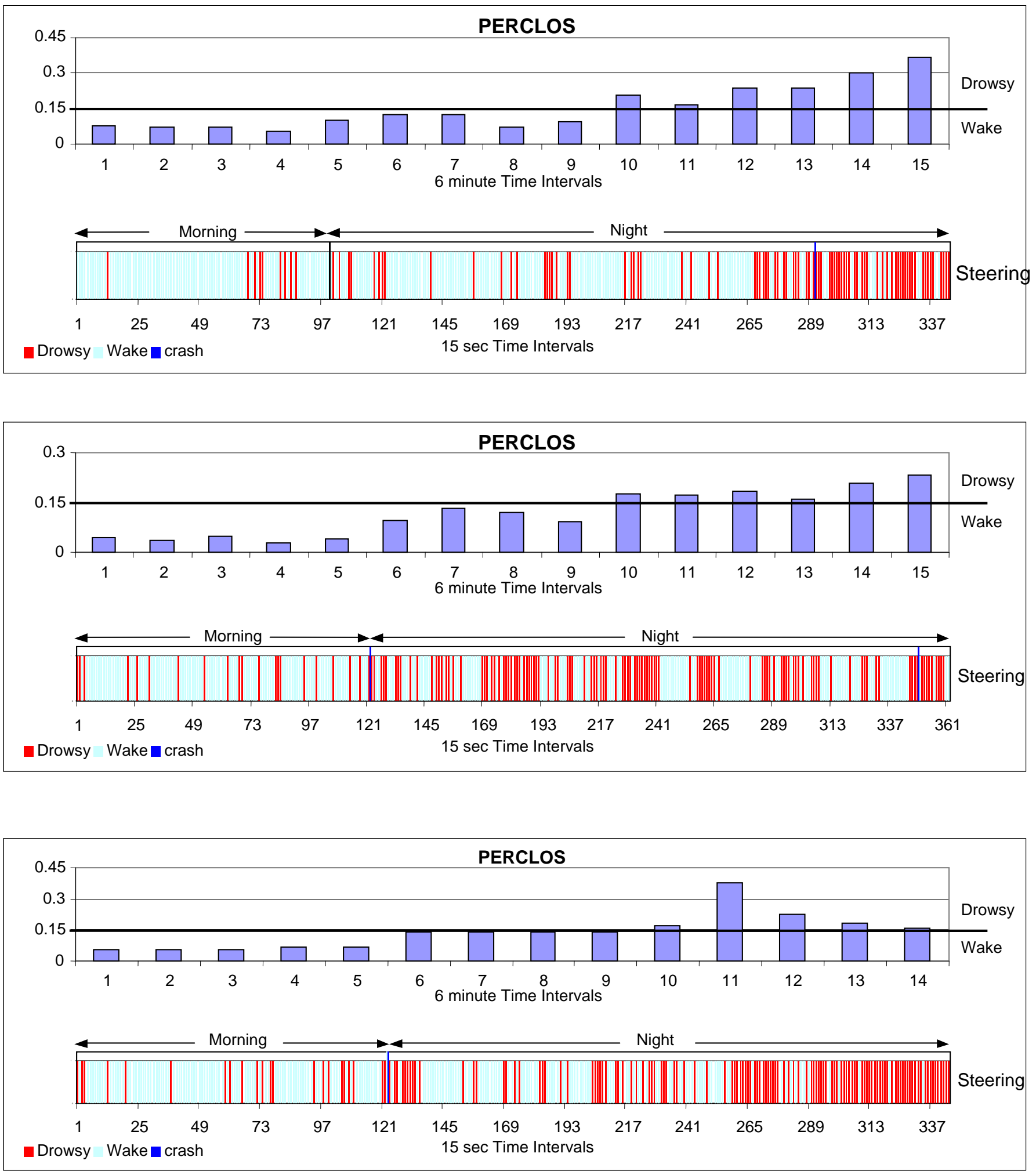

Figure A.6: Comparison with PERCLOS (6-min time intervals) 


\section{ANN TRAINING (STEERING + EYE)}

Data from 10 subjects, which make a total of 1,600 driving intervals (as previously explained), was randomized and then divided into three separate files:

- Training file, which includes a set of 900 input-output exemplar vectors

- Cross validation file, which includes a set of 200 input-output exemplar vectors

- Testing file, which includes a set of 500 input-output exemplar vectors

These files contain input vectors $\boldsymbol{X}(\boldsymbol{n})$ and output vectors $\boldsymbol{D}(\boldsymbol{n})$, each representing 15 seconds of driving. Table A.4 and Figure A.7 show a portion of the input file.

Table A.4: ANN performance during training (steering+eye)

\begin{tabular}{|c|c|c|}
\hline Best Networks & Training & Cross Validation \\
\hline Epoch \# & 1,000 & 764 \\
\hline Minimum MSE & 0.096 & 0.0773 \\
\hline Final MSE & 0.096 & 0.078 \\
\hline
\end{tabular}

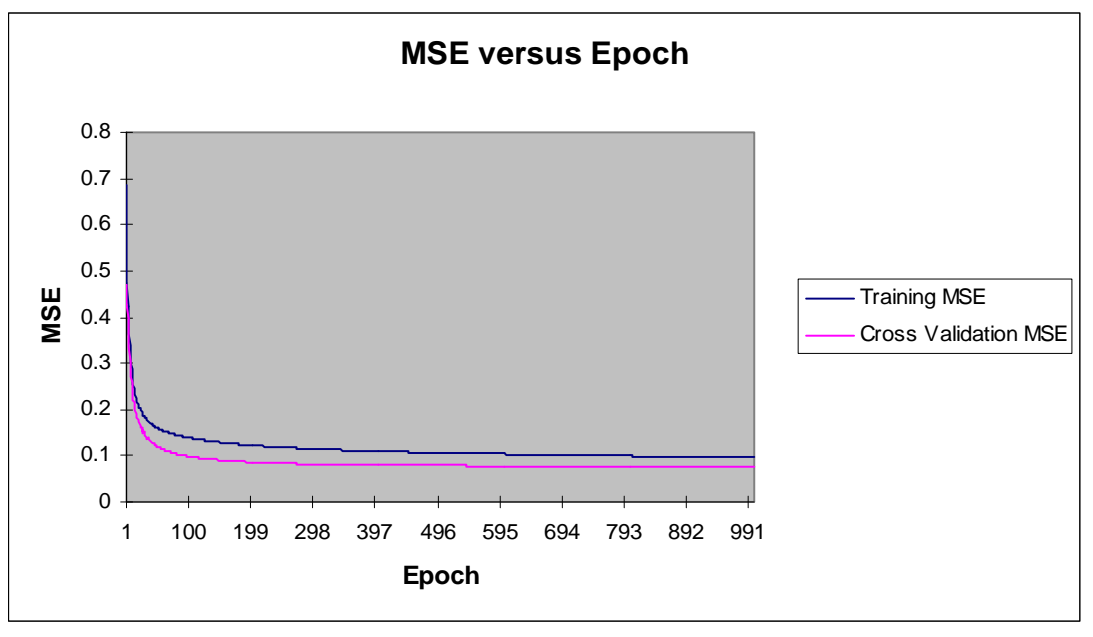

Figure A.7: ANN training performance 


\section{ANN TESTING (STEERING + EYE)}

The network classified the test data into wake and drowsy driving intervals with an accuracy of more than $92 \%$. This classification is shown in Table A.5.

Table A.5: Test results (steering+eye)

\begin{tabular}{|c|c|c|}
\hline \multirow{2}{*}{ OUTPUT } & \multicolumn{2}{|c|}{ DESIRED } \\
\cline { 2 - 3 } & WAKE & DROWSY \\
\hline WAKE & 179 & 16 \\
\hline DROWSY & 14 & 191 \\
\hline \% CORRECT & 93 & 92.3 \\
\hline
\end{tabular}

The network correctly identified 191 out of a total of 207 drowsy intervals, an accuracy of $92.4 \%$. There were 14 "false alarms", intervals that were in wake class but were misclassified as drowsy. Also, 16 intervals that were in the drowsy class were misclassified by the network as wake.

\section{CRASH PREDICTION (STEERING + EYE)}

All drivers who completed the night driving session, with exception of subject 09 , fell asleep while driving and crashed the vehicle one or more times. The network identified sleep patterns before the crash with an accuracy of 100 percent. Figures A. 2 - A.5 show that in all these cases, the ANN is continuously identifying drowsy intervals before a crash.

\section{TESTING ALL SUBJECTS (STEERING + EYE)}

Data from both morning and night experimental driving sessions of all the subjects was presented to the ANN, one subject at a time. The ANN output, for both morning and night experimental driving sessions, of all the subjects is shown in Figures A.2 - A.5. These figures show that during the morning driving sessions the ANN identified very few drowsy intervals and, therefore, few false alarms. There are a total of 75 false alarms (i.e., $6 \%$ of the total morning driving intervals). During the night driving sessions, when the drivers were sleep deprived, the ANN identified the majority of the intervals as drowsy. As shown in Table A.6, The ANN identified 60 percent of the total night driving intervals as drowsy. 
Table A.6: Percentage of drowsy intervals identified by ANN

\begin{tabular}{|l|c|c|c|c|c|c|c|c|c|c|c|}
\hline \multirow{3}{*}{ Session } & \multicolumn{10}{|c|}{ Subject Number } \\
\cline { 2 - 12 } & $\mathbf{1}$ & $\mathbf{2}$ & $\mathbf{3}$ & $\mathbf{4}$ & $\mathbf{5}$ & $\mathbf{6}$ & $\mathbf{7}$ & $\mathbf{8}$ & $\mathbf{9}$ & $\mathbf{1 0}$ & $\mathbf{1 1}$ \\
\hline Morning & $9.5 \%$ & $9.8 \%$ & $12.2 \%$ & $12.7 \%$ & $8.1 \%$ & $5.7 \%$ & $2.0 \%$ & $1.6 \%$ & $8.1 \%$ & $6.5 \%$ & $0.0 \%$ \\
\hline Night & $60.4 \%$ & $47.2 \%$ & N/A & $73.3 \%$ & $63.0 \%$ & $47.1 \%$ & $97.6 \%$ & $52.8 \%$ & $85.0 \%$ & $57.2 \%$ & $41.2 \%$ \\
\hline
\end{tabular}

Although the eye closure data was used in training the ANN, a comparison of the ANN output and PERCLOS show a high correlation. In Figure A.8, each bar represents the percentage of those driving intervals, which were classified as drowsy by the ANN and also for which the value of the corresponding eye closure interval is above the drowsy threshold. The threshold value used here is the value of PERCLOS that is $30 \%$ higher then the average value of the morning driving session.

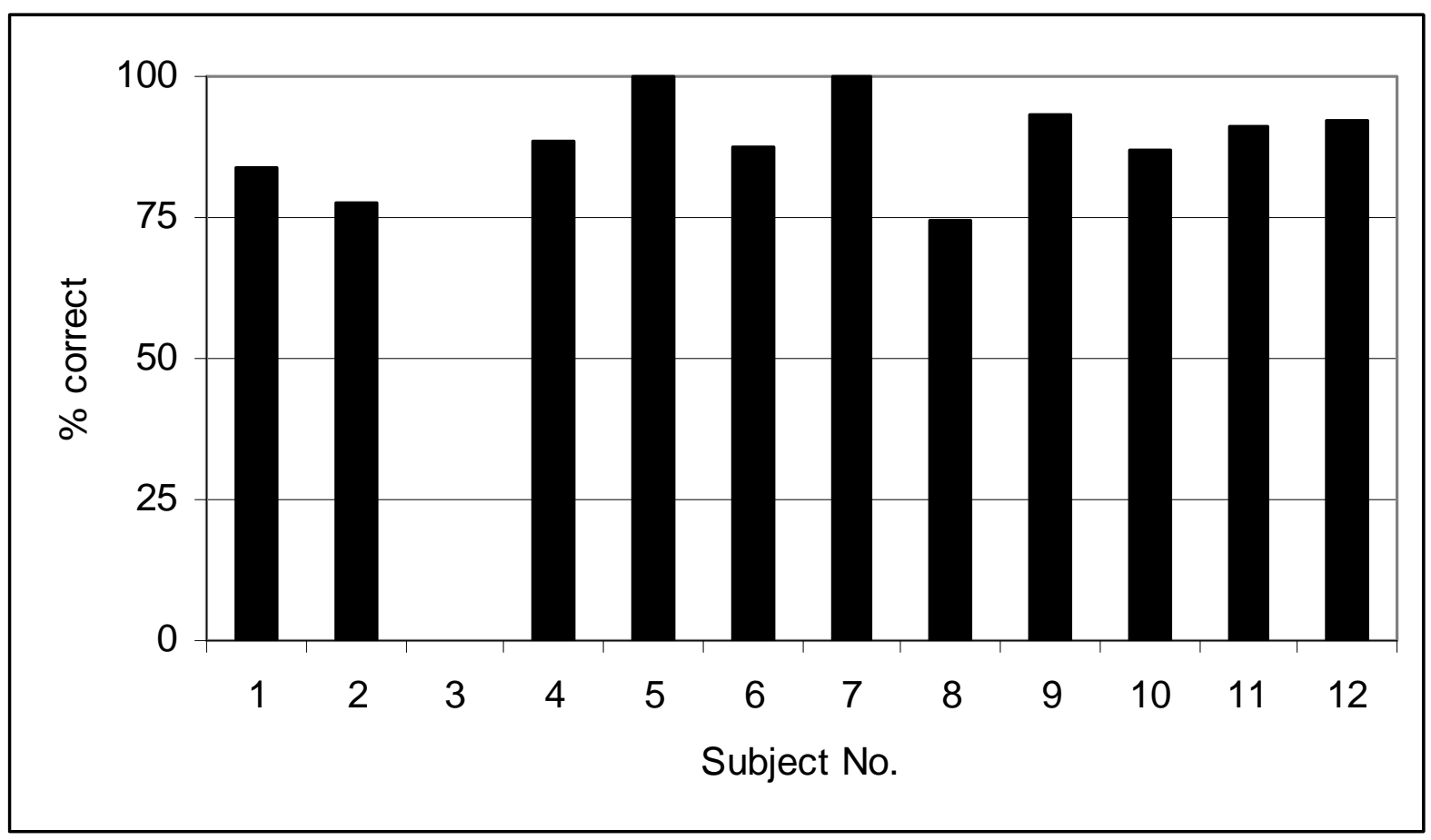

Figure A.8: PERCLOS comparison 


\section{APPENDIX B. DESIGN, FABRICATION, AND ADAPTATION OF HARDWARE ELEMENTS FOR CISR TRUCK SIMULATOR}

This appendix provides a detailed technical specification of the CISR Truck Simulator. Specific items that are discussed include the steering algorithm, the steering system mounting, the truck cabin preparation, the display screens, the gearbox mounting, the truck cabin pedals, the cabin adaptation and instrument interfacing, the sound systems, and the driving scenario development.

\section{STEERING ALGORITHM/MODEL}

The steering system is based on the INRETS haptic steering feedback algorithm. The Mohellebi and Espié steering algorithm is based on models used in robotic tele-operation. This model considers that the torque generated on the steering shaft is equivalent to the torque generated on the wheel pivot. Also, taking into account the induced rolling effect, the wheel torque is given by:

$$
\Gamma_{\text {wheel }}=a M \gamma-b M g \theta
$$

where $g$ is the gravity, $M$ is the vehicle mass, $a$ and $b$ are vehicle specific constants, $\theta$ is the roll angle, and $\gamma$ is the lateral acceleration. The steering shaft torque is then given by:

$$
\Gamma_{\text {shaft }}=\frac{\Gamma_{\text {wheel }}-K_{\alpha} \alpha}{G}
$$

where $\alpha$ is the steering angle, $K_{\alpha}$ is the steering shaft linkage stiffness, and $G$ is the gear reduction ratio (approximately 20 ).

The adopted closed loop controller is shown in Figure B.1. $\alpha_{d}$ is the desired steering angle, $\tau_{\mathrm{d}}$ is the human applied steering torque, and $\tau_{\mathrm{s}}$ is the motor measured torque.

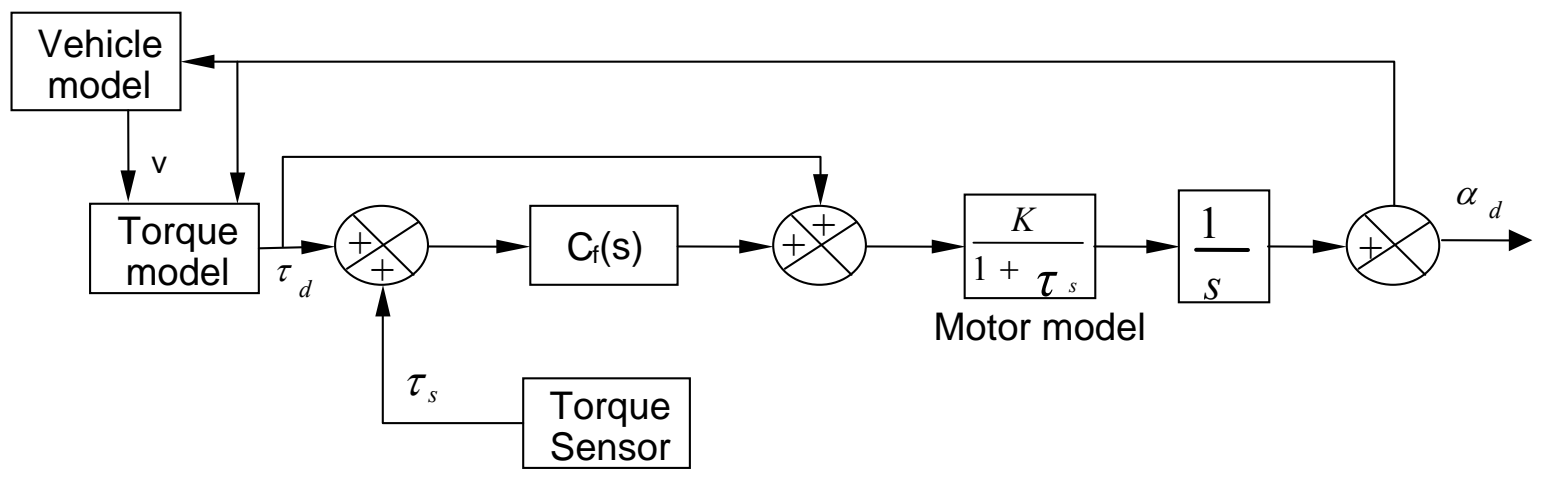

Figure B.1: Closed-loop controller 


\section{STEERING SYSTEM MOUNTING}

In order to increase the realism inside the truck cabin, only the original components of the truck steering system are utilized. The original steering wheel, steering shaft, universal joints, and mountings are used. Additional steering hardware is installed in the front under the hood area, not visible to the driver. A steel frame was designed and fabricated to hold the steering hardware. This support plate was carefully designed to hold the steering shaft at the proper angle aligned with the steering. This assembly is designed to provide flexibility for adjusting the angle and positioning of the hardware. When the whole system was finely adjusted and tested, it was welded together and then to the truck cabin body. Figure B.2 shows details of the steering assembly, the support steel frame attached to the truck cabin, and the steering shaft connection elements.

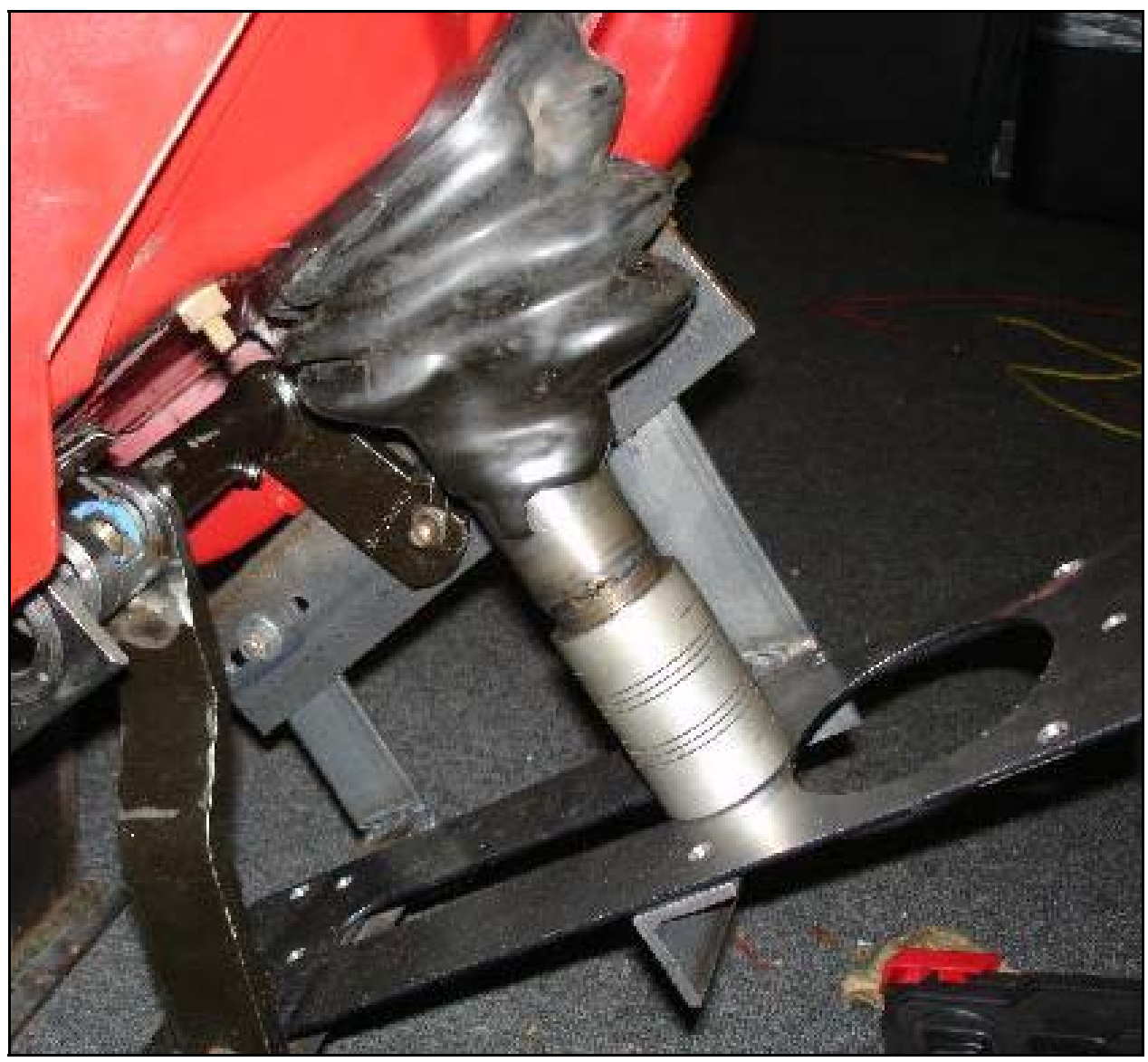

Figure B.2: Steering assembly, steering system mounting 


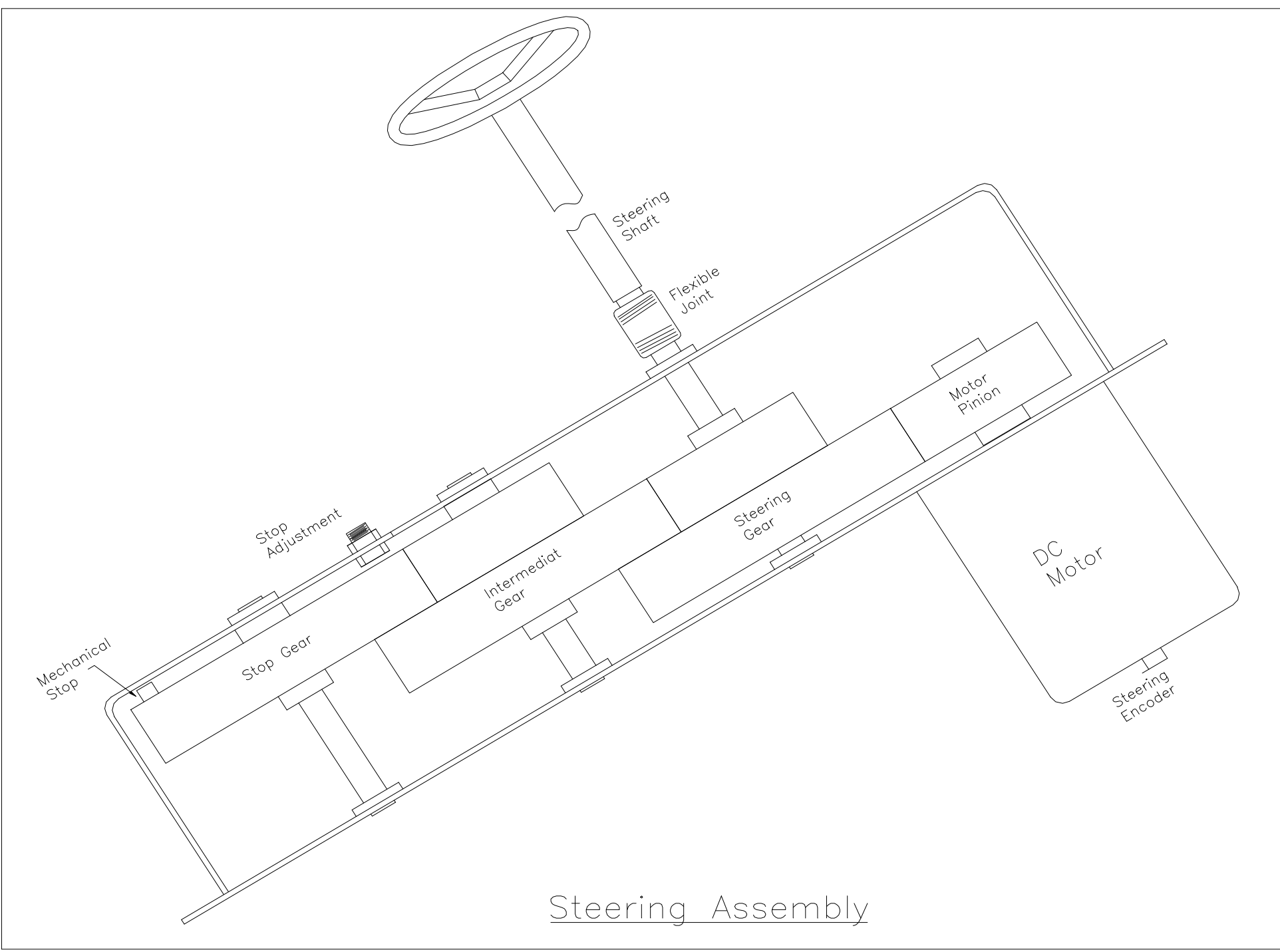

Figure B.2: (continued) Steering assembly, steering system mounting frames and detail of steering shaft connection 


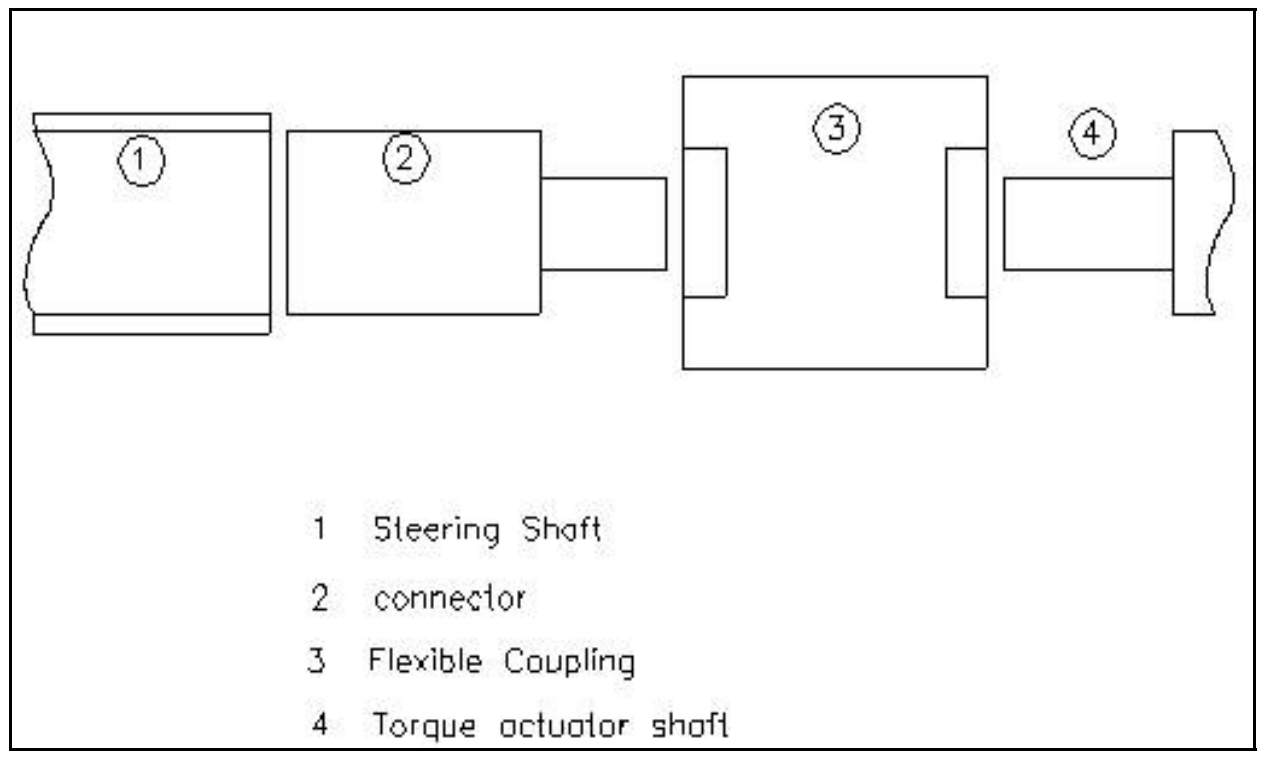

Figure B.2 (continued): Steering assembly, steering system mounting frames and detail of steering shaft connection

\section{TRUCK CABIN PREPARATION}

The truck cabin and its hood were acquired from Pennsylvanian-based William Cohen \& Sons Inc. The cabin and hood was shipped to the GWU campus in Ashburn, VA, see Figure B.3.

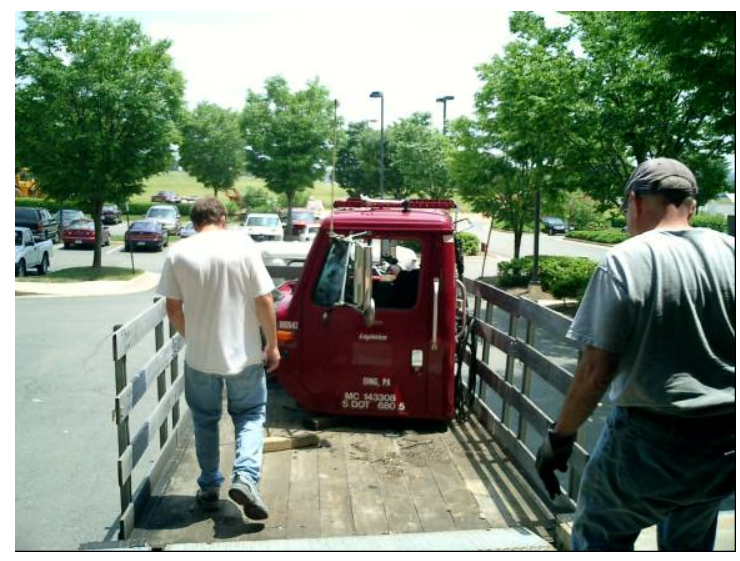

Figure B.3: Truck cabin being delivered

The truck cabin was temporary placed on four adjustable jacks to be able to fine-tune its final location and height. This low-cost solution provided both a sturdy foundation for the truck cabin and some flexibility in its height adjustment. CISR then designed and fabricated a rectangular steel frame base for the truck cabin after all the fixed position 
was finalized. Two longitudinal channel steel sections were connected across by two right angle steel sections, which were supported by four steel legs. The whole assembly was bolted to the truck cabin. This arrangement provided durable support for the cabin and other equipment, including the gearbox and steering torque system. Figure B.4 shows the cabin mounted on temporary adjustable jacks and the drawing of the finalized cabin base/foundation.
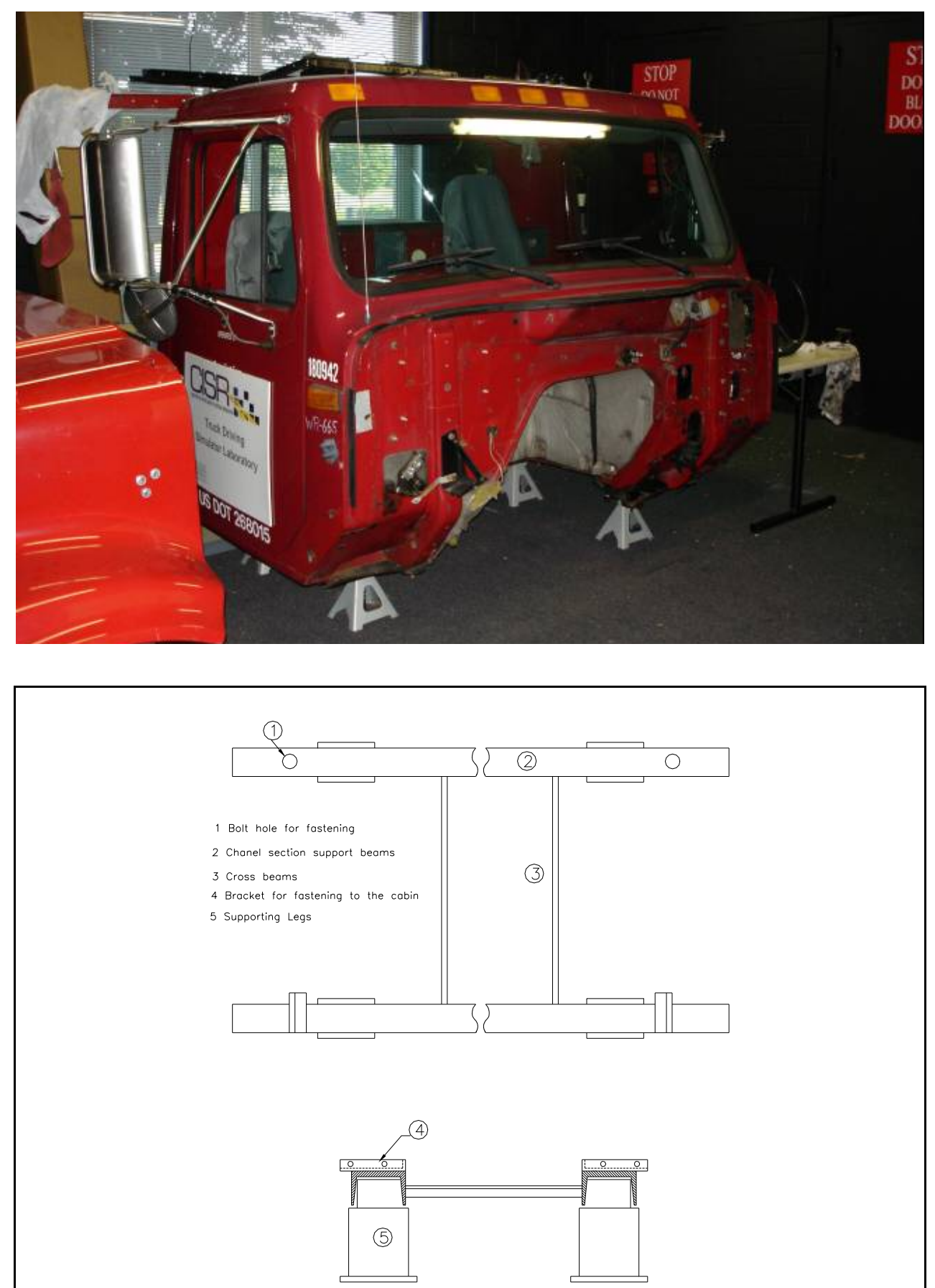

Figure B.4: Truck cabin on temporary jacks and final support base 
Most of the simulator control elements, such as the steering feedback system, brake and clutch position sensors, data acquisition system, etc., are located in the front area under the hood. To make these elements more easily accessible, CISR designed and fabricated a movable platform to mount the hood.

The cabin was then prepared for instrumentation and integration. The inside of the cabin was entirely disassembled, all the unwanted material was striped off, and all components were cleaned, labeled, and reintegrated to the cabin, as shown in Figure B.5.
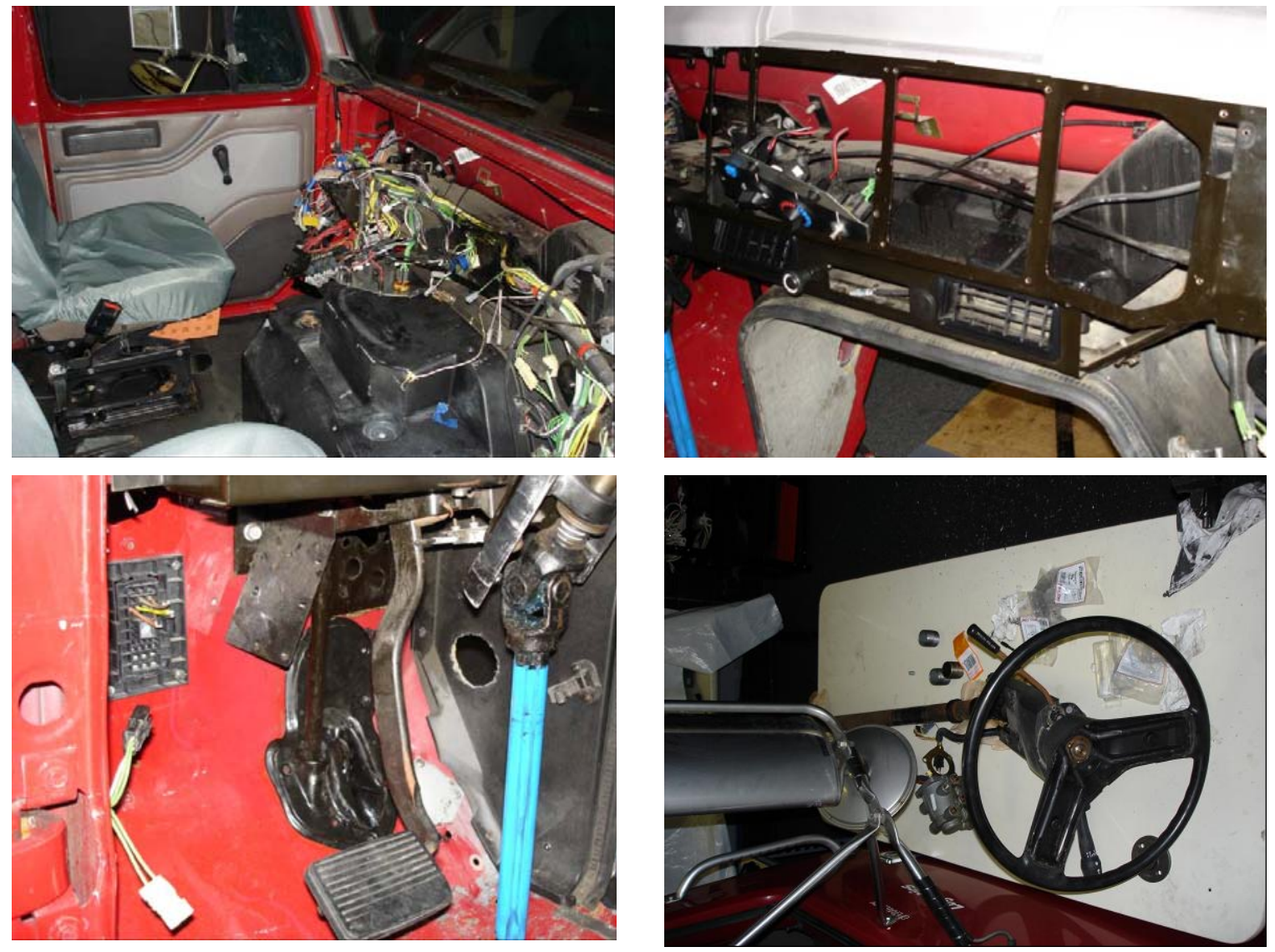

Figure B.5: Dashboard wiring, auxiliary controls removed, pedals disassembled, and steering components removed

\section{DISPLAY SCREENS}

For the forward projection system, CISR designed three flat screens. The front projected images can be up to $9 \mathrm{ft}$ wide by $6 \mathrm{ft}$ high. To allow some flexibility in the image height adjustment, each front screen is $9 \mathrm{ft}$ wide by $6.5 \mathrm{ft}$ high. When placed together in the available $19.5 \mathrm{ft}$ wide room, they provide a field-of-view of 135 degrees. 
These screens were custom-designed by CISR and were fabricated in-house. Each of these three screens consists of the following parts:

- Supporting Frame: The dimensions of the frame are $13 \mathrm{ft}$ high and $6 \mathrm{ft}$ wide and is fabricated using steel pipes and the frame is secured to the wall by adjustable steel fasteners. The frame is mounted on cast iron flanges secured to the ground. Specially designed aluminum blocks are attached to accommodate the screen, which is placed at a height of $3 \mathrm{ft}$ from the ground.

- Screen Frame: Figure B.6 shows the detail design of the screen frame. The rectangular screen frame is fabricated with rectangular steel tube sections. These sections were cut to size, drilled, and fastened to the aluminum blocks in the supporting steel frame. Tubular sections provide a very rigid and lightweight design.

- Screen Fabric: A special screen fabric was wrapped around the screen frame to provide a flat surface for display. This fabric is custom-ordered to fit the screen dimensions and has a white mat finish and a non-reflective surface. Small gage wire rope is used to wrap the fabric around the frame. The whole design is such that only the front white surface is visible to the viewer; everything else is behind the screen.

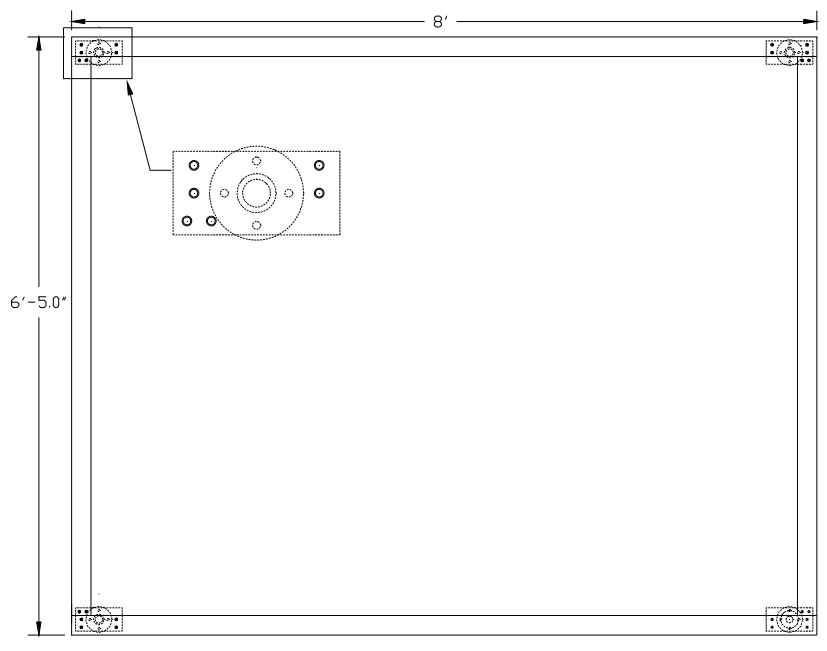

Figure B.6: Front projection screen

Figure B.7 shows the completed frame structures in final location. 

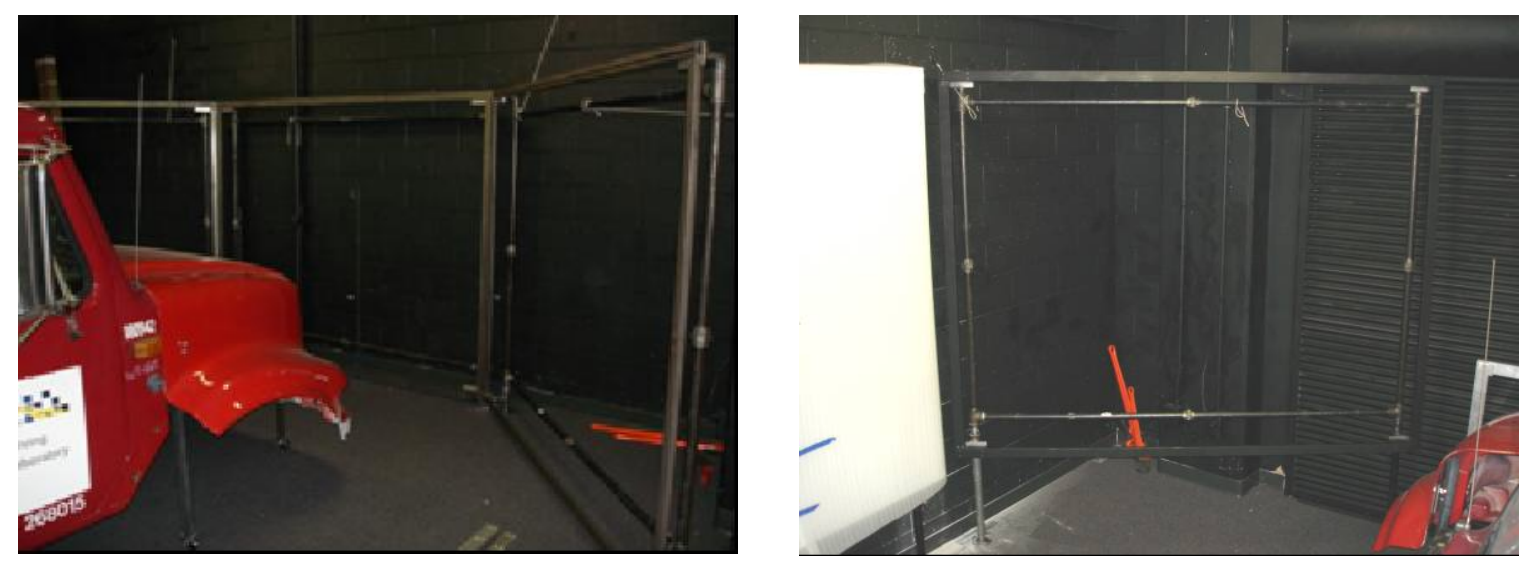

Figure B.7: Front screens frame structure and support

The design used for rearview mirrors is similar to those for the forward projection screens. The overall dimension of these screens is $4 \mathrm{ft}$ wide by $4 \mathrm{ft}$ high. The frames are made out of aluminum, directly hung from the top at two points, and do not require an additional supporting frame. Steel beams initially used for the truck roof equipments (i.e., horn, lights, etc.), were modified to provide adapted support to hang the rear screens' frames. The special fabric for these screens allows for both front and back projection. Due to light construction and easy mounting they are ideal for use in such an environment. Figure B.8 shows the completed screen.

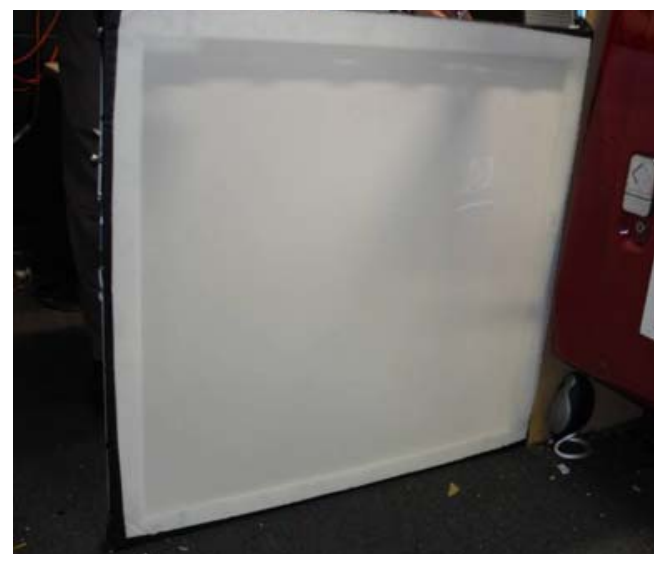

Figure B.8: Rear screens

\section{GEARBOX MOUNTING}

To mount the gearbox system in the right position, CISR designed and fabricated a steel frame and housing. Many considerations were taken into account to make sure that any modification did not change the original character of the truck cabin interior. Figure B.9 

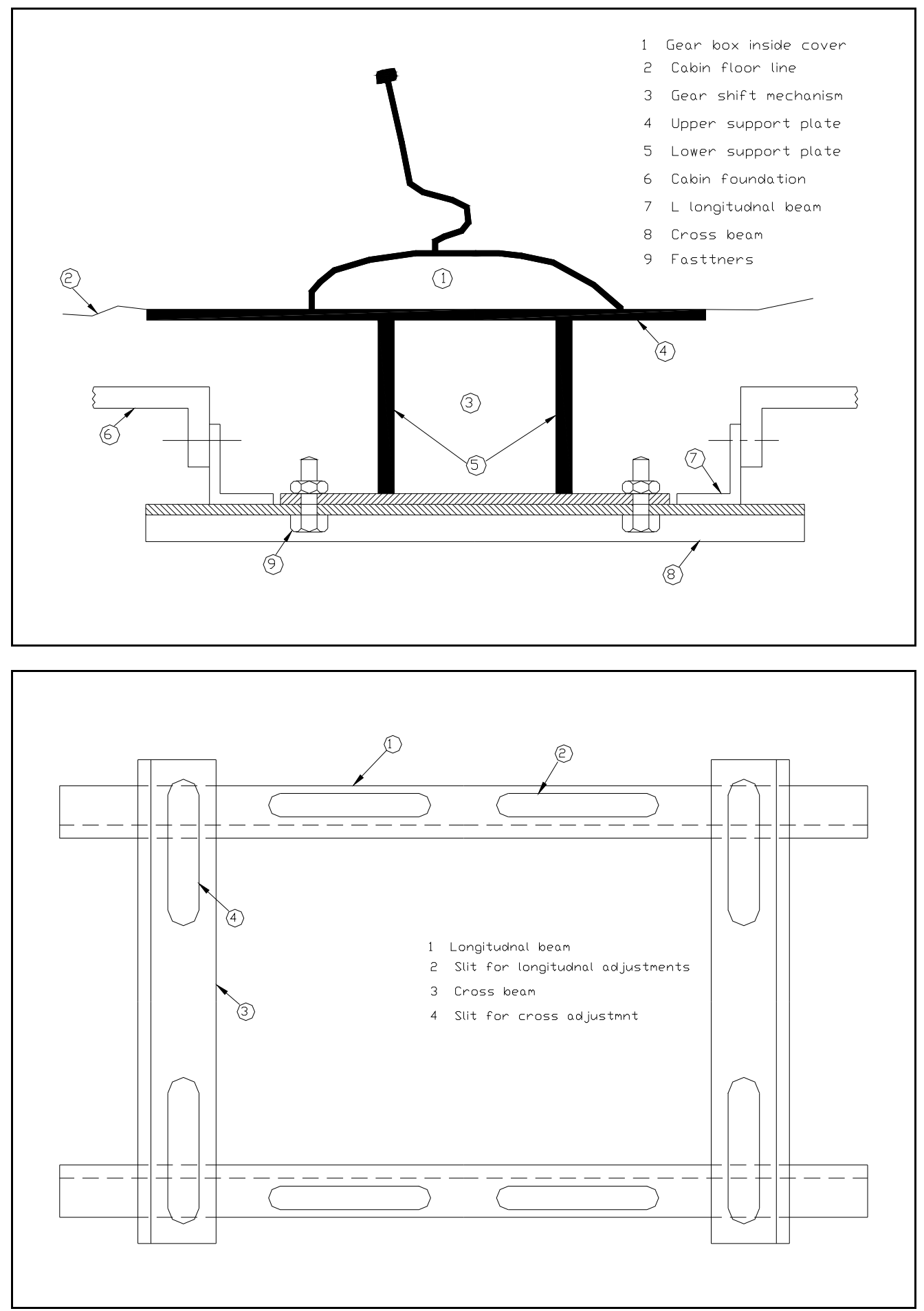

FigureB.9: Final assembly and mounting frame for the gearbox system

shows the detailed design of the mounting frame. A rectangular frame was constructed using four right angle steel sections. Two longitudinal sections were welded to the cabin support frame. The other two sections were bolted to the lower support plate of the gearbox. These two sections were then bolted to the welded sections. Longitudinal rectangular slots were machined into all the four sections instead of drilling round holes. 
This provides a mechanism for adjusting the position of the gearbox inside the cabin. The whole frame was lowered so that the top plate is at the same level with the cabin floor.

The gear change lever (stick) was modified to adapt to the truck cabin configuration. It was redesigned by changing the angle and length, for easy reach by the driver. The final position of the shift lever was determined through subjective evaluation by various CISR members. Because of the adjustable design of the mounting frame, it was possible to fine-tune the final position of the stick. The original cabin floor had to be cut to fit the new gearbox assembly.

In the real truck, clutch and brake pedals were connected to hydraulic systems for controlling gear shifting and braking. In the driving simulator, these hydraulic elements were removed and replaced with a different mechanism that reproduces the feel of the pedals. Simple passive systems, based on spring load and small hydraulic resistance, were designed, and these systems produced good results once calibrated. The design, modification, and operation of the new simulator pedal systems are discussed in the following sections.

\section{Brake Pedal}

The original brake pedal of the truck was modified. Figure B.10 details the construction of the new brake pedal pressure feel system. The braking pressure or feeling in the pedal is created through a combination of hydraulic and spring mechanism. Pressing on the foot pedal (1) causes the roller (3) to press against the rubber housing (4). The compression spring (5) and piston (6) creates the resistance force against the pedal movement. The initial pressure feel is generated by the spring (3), further movement of the pedal causes the rubber housing to press against the hydraulic cylinder. This combination of spring and hydraulic pressure creates a very realistic feeling in the pedal. The hydraulic cylinder from the truck original braking system is used for a more realistic feeling.

The pedal motion is transmitted to the controller card through an optical encoder. Figure B.11 shows a detailed diagram of the encoder mounting mechanism. The pivot shaft (2) and pedal hub (3) are joined together by retaining pins (6). The pivot shaft (2) is supported at two points by steel bearings housed in the mounting bracket. The encoder (7) is screwed to support plate (5), which is welded to the mounting bracket. The encoder shaft is connected to the pivot shaft through a flexible coupling (4). Pushing on the foot pedal (1) causes the pivot shaft to rotate, which in turn causes the encoder to rotate, allowing for the detection of movement in the pedal. 


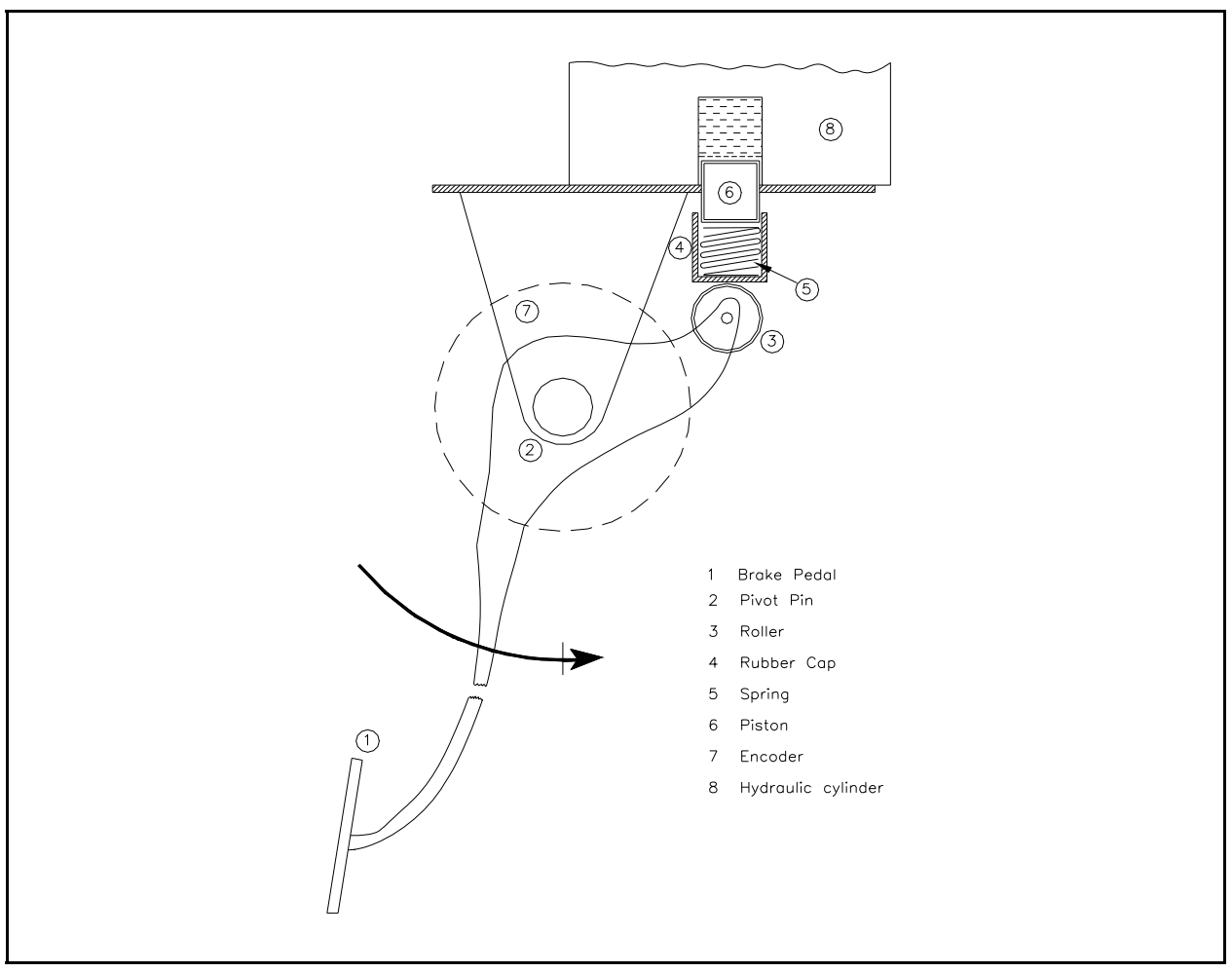

Figure B.10: Modified brake pedal

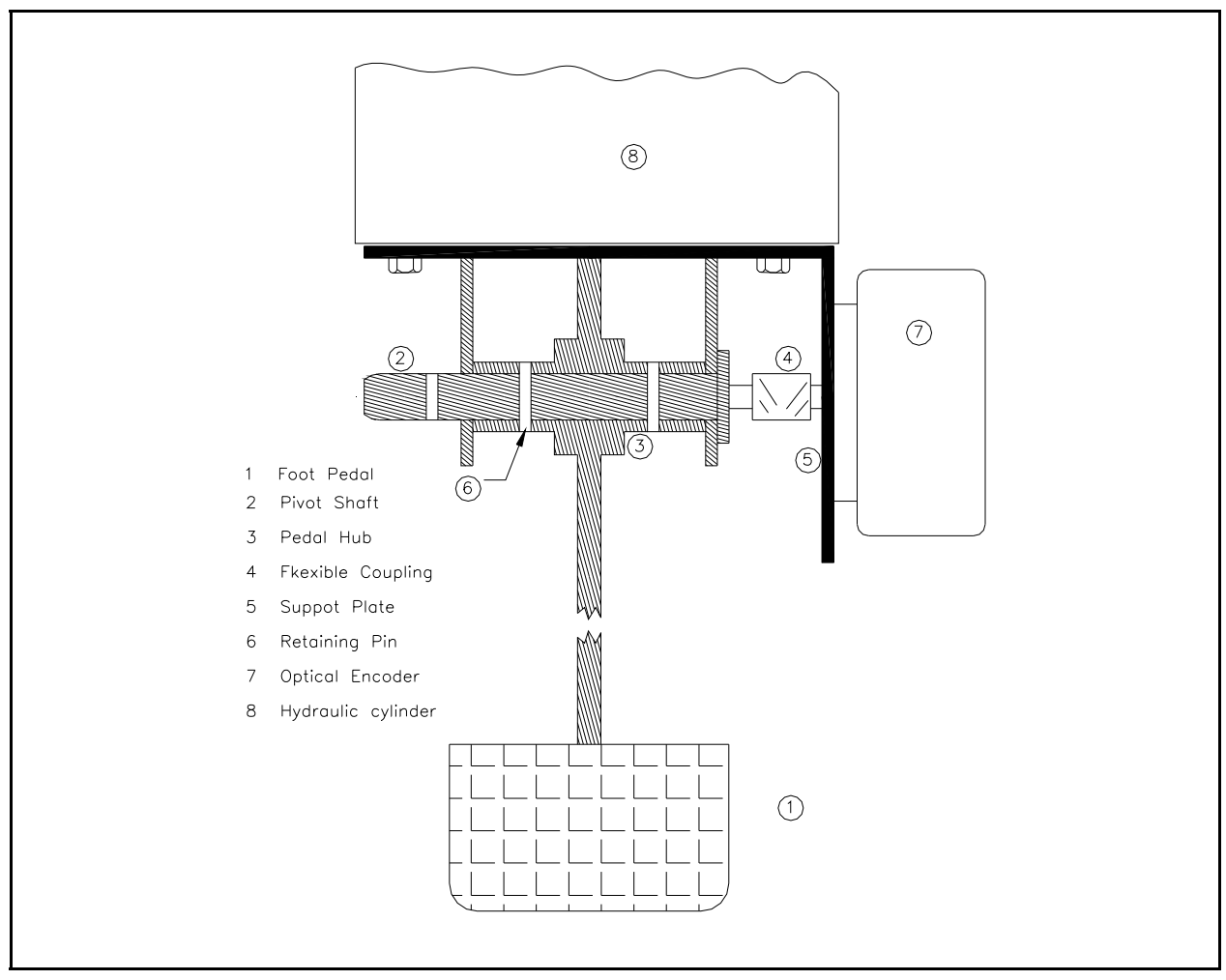

Figure B.11: Brake pedal encoder support assembly 


\section{Clutch Pedal}

The clutch pedal and its mountings inside the cabin are all original. The modifications and encoder housing are located outside the cabin in the front firewall area. Figure B.12 depicts the construction and operation of the new clutch pedal pressure feel system. The feeling of clutch action in the pedal is created through a spring lever mechanism.

Pressing on the foot pedal (1), which swivels on pivot pin (2), causes a connecting rod (6) to raise the crank lever (4). This motion turns the two crank levers (4) in counterclockwise direction and pulls against the extension spring (8). This spring pressure creates the resistance or load in the pedal.

Figure B.13 shows a detailed diagram of the encoder mounting mechanism. Pressing the clutch pedal causes hollow tube (8) to rotate about pivot shaft (2). A hollow cylindrical connector (6), which is welded to a hollow tube (8), transmits this rotary motion to the encoder (7) through a flexible connector (1). A support bracket (3) holds the encoder at one end of the tube (8). The other end of the hollow tube is bolted to the truck cabin (5). Pushing on the foot pedal causes the pivot shaft to rotate, which in turn, causes the encoder to rotate, allowing for the detection of movement in the pedal.

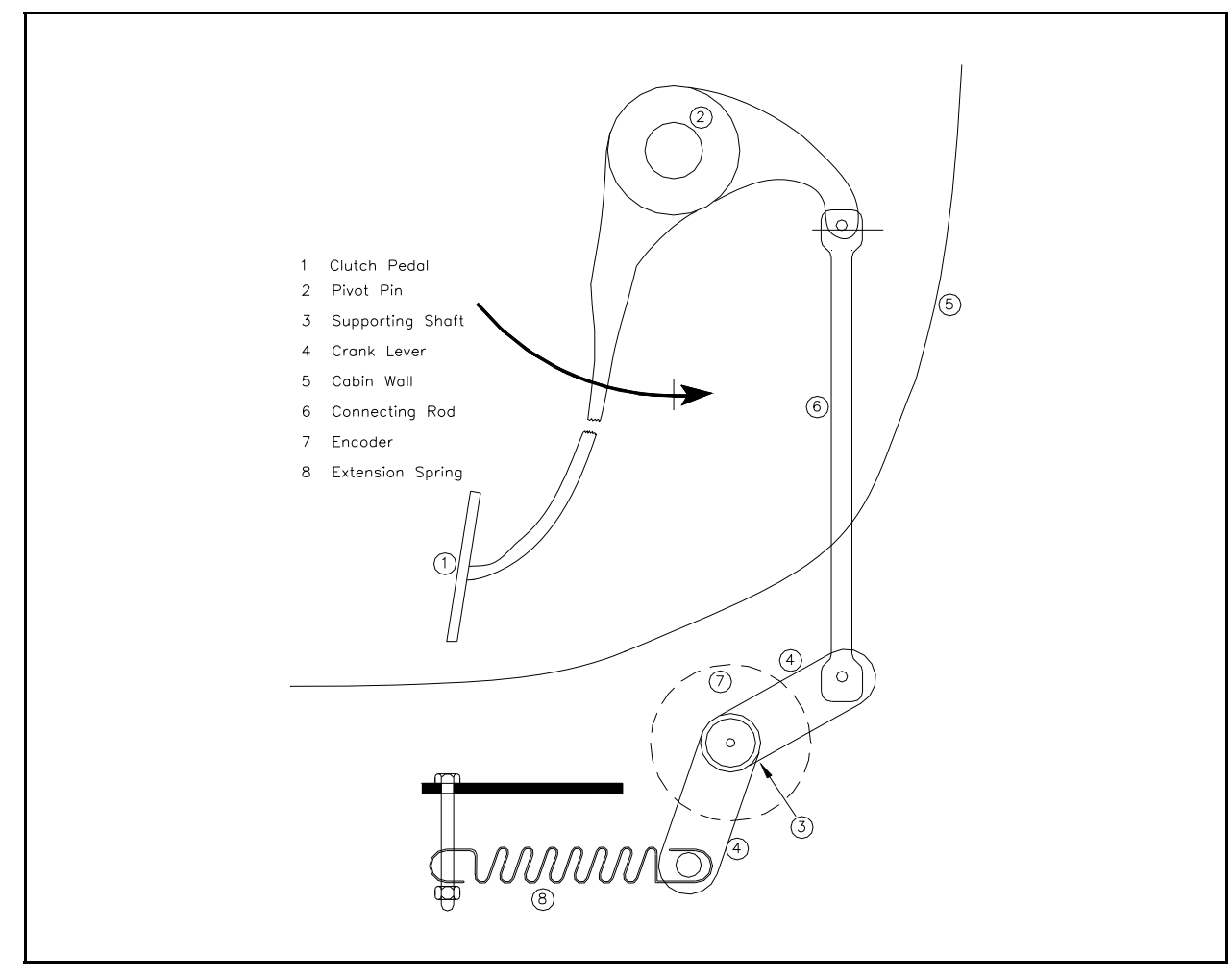

Figure B.12: Modified clutch pedal 


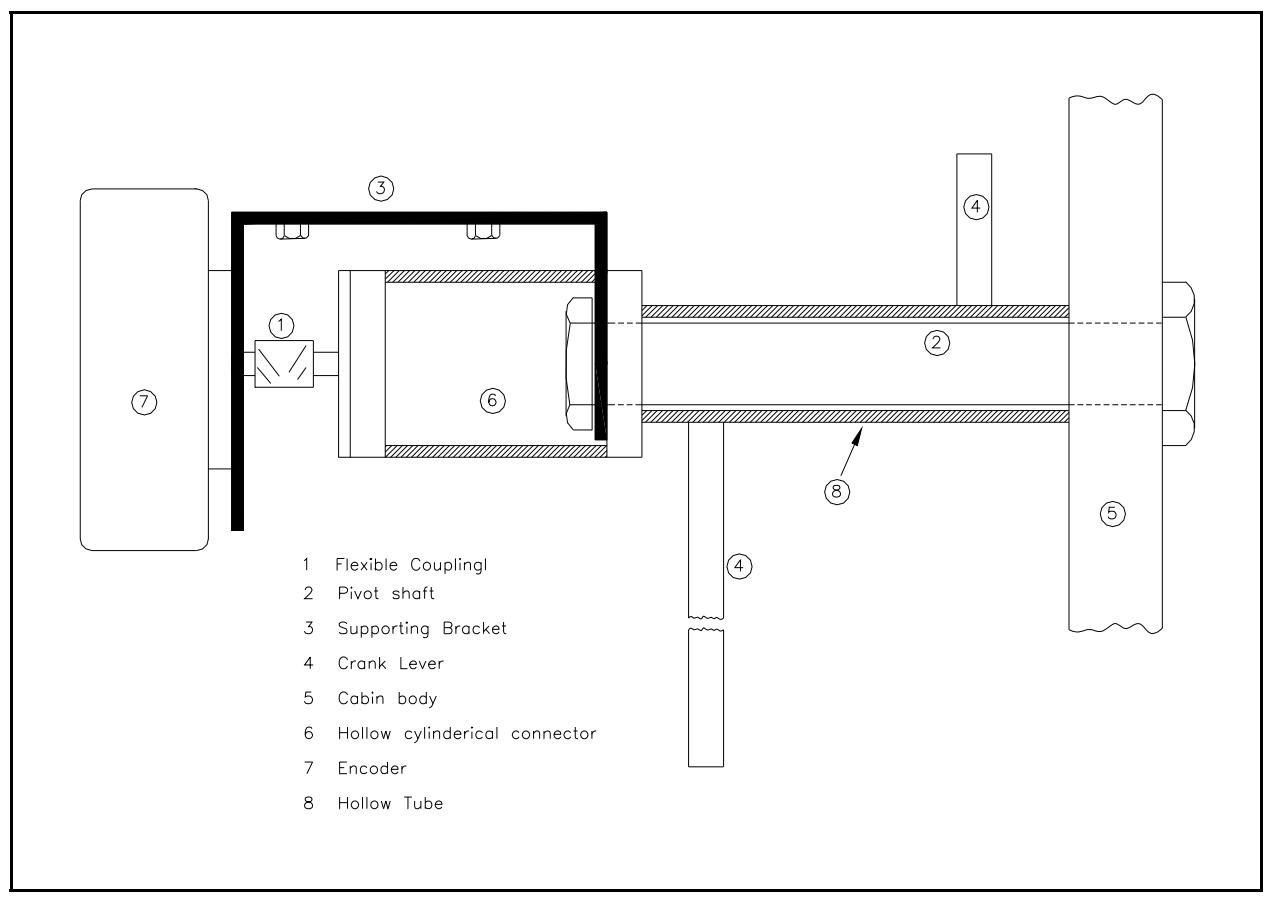

Figure B.13: Clutch pedal encoder mounting assembly

\section{Throttle Pedal}

The throttle pedal resistance mechanism is generated with the original truck throttle pedal system. Figure B.14 shows the working of the throttle pedal pressure feel system and the

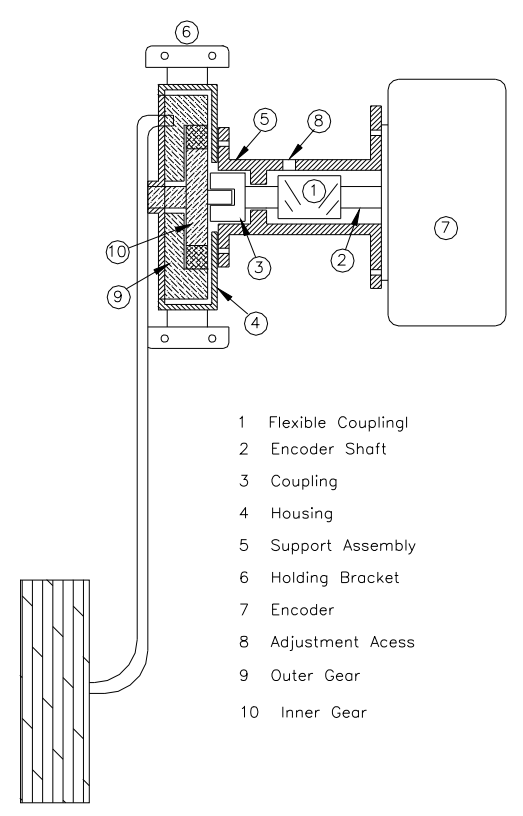

Figure B.14: Throttle pedal and encoder assembly 
throttle encoder mountings. A torsion spring and gear mechanism produces resistance in the pedal. Pressing the throttle pedal will turn a spring-loaded plastic outer gear (9) that meshes with inner gear/sector (10). This gear and spring mechanism is enclosed by an aluminum housing (4). Circular motion of the inner gear is transmitted to the encoder (7) through a small aluminum coupling (3) and a flexible coupling (1). A support assembly (5) supports the encoder and both couplings. The whole assembly is attached to the cabin through a steel bracket (6).

\section{CABIN ADAPTATION AND INSTRUMENT INTERFACES}

Gauges (speedometer and tachometer gauges), instruments (turning indicators, vehicle lights, start key), controls (pedal optical encoders), and switches (cabin dome-light, radio, etc.) of the truck cabin were connected to the simulation software system for real-time operation.

To prevent potential voltage losses in the optical encoders and connecting wires, a voltage adaptation layer was designed. This unit also enables secure wiring between vehicle cabin digital signals and inputs of the CEGI card, as shown Figure B.15.
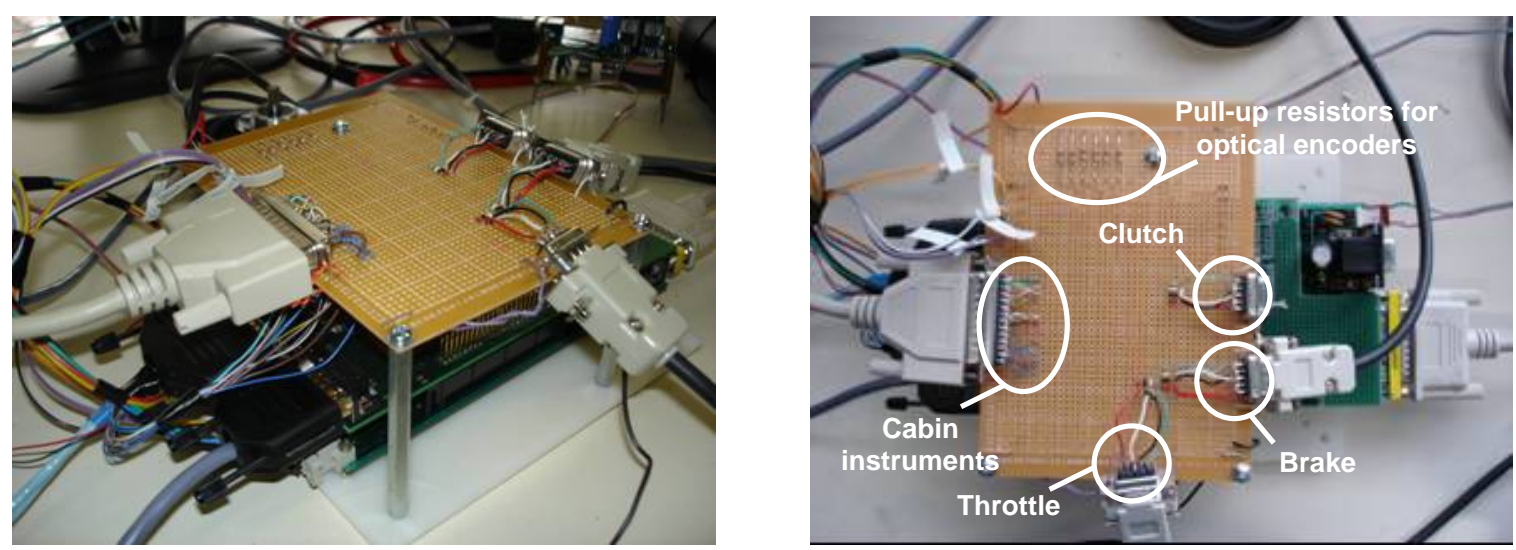

Figure B.15: Connection card for pedals optical encoders and cabin digital inputs

Because the CEGI card is operating under 5 volts, an additional layer of adaptation was required to manage the 12 volt cabin instrument, including the dashboard indicators and warnings, cabin dome light, radio, etc. A relay card was designed to switch from 5 volts to 12 volts as illustrated in Figure B.16. 

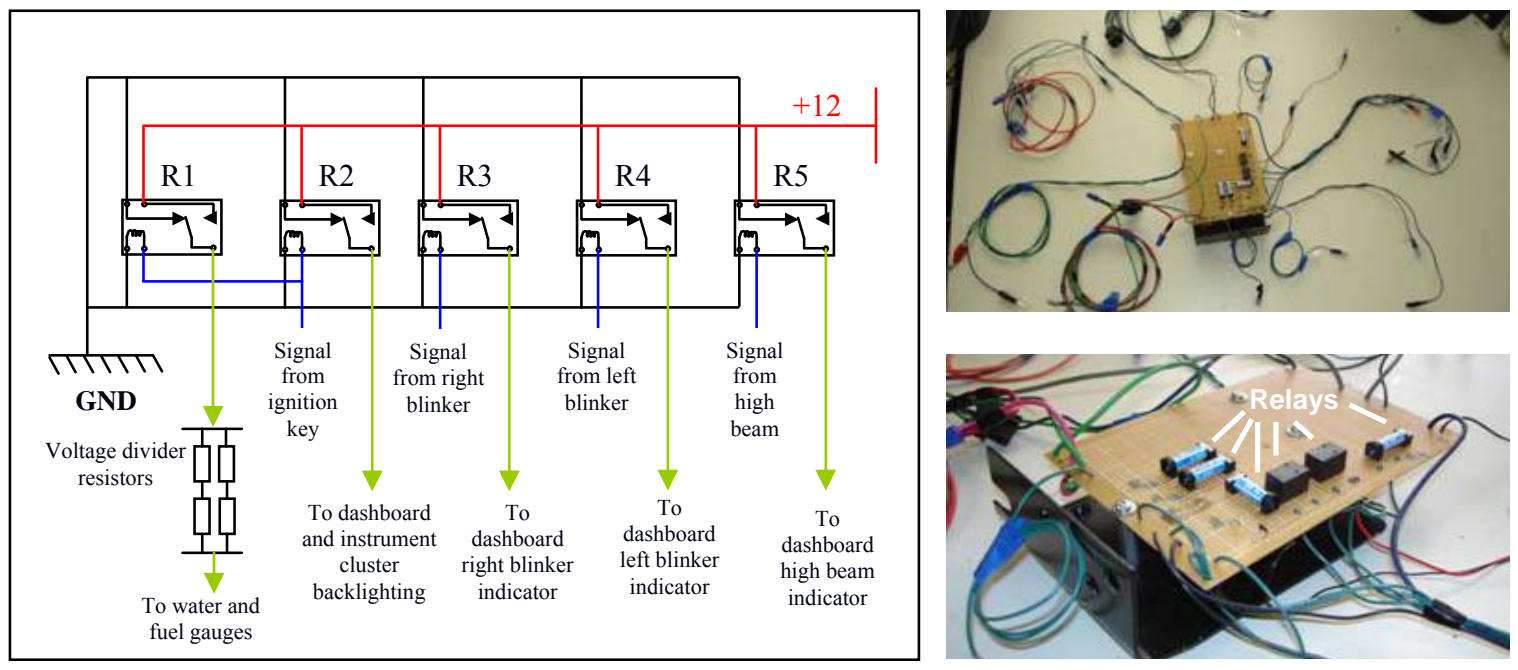

Figure B.16: Circuit diagram of the cabin relay card and finalized electronic unit

\section{SOUND SYSTEM}

Sound plays an important role in the realism of driving simulation. Wind and engine noise contribute to fatigue in drivers who have logged many hours. Sirens and horns can divert driver's attention away from the task at hand. Traffic noise can also affect a driver's state of being and decision-making. Squealing tires are indicators that the car is being pushed towards its handling limits. The CISR simulator sound system is capable of generating both the traffic sounds (i.e., other vehicle sounds, engine/acceleration sound, braking noise, etc.) and the vibrations transmitted to the vehicle cabin during driving.

The human brain is able to determine the source of a sound it perceives by analyzing the input it receives from each ear. To create an immersive experience, sounds in the virtual environment must replicate this physical phenomenon. That is, they must be given a sense of locality. To do this, the simulation sound generator must manipulate the speaker outputs to trick the brain into perceiving that the sound is coming from a specific location in space.

A multi-channel sound system composed of five speakers and a subwoofer is strategically placed in the cabin to optimize the global sound rendering. The subwoofer is attached to a steel plate, which is bolted to the truck cabin to create vibrations in the cabin during simulations, see Figure B.17. 


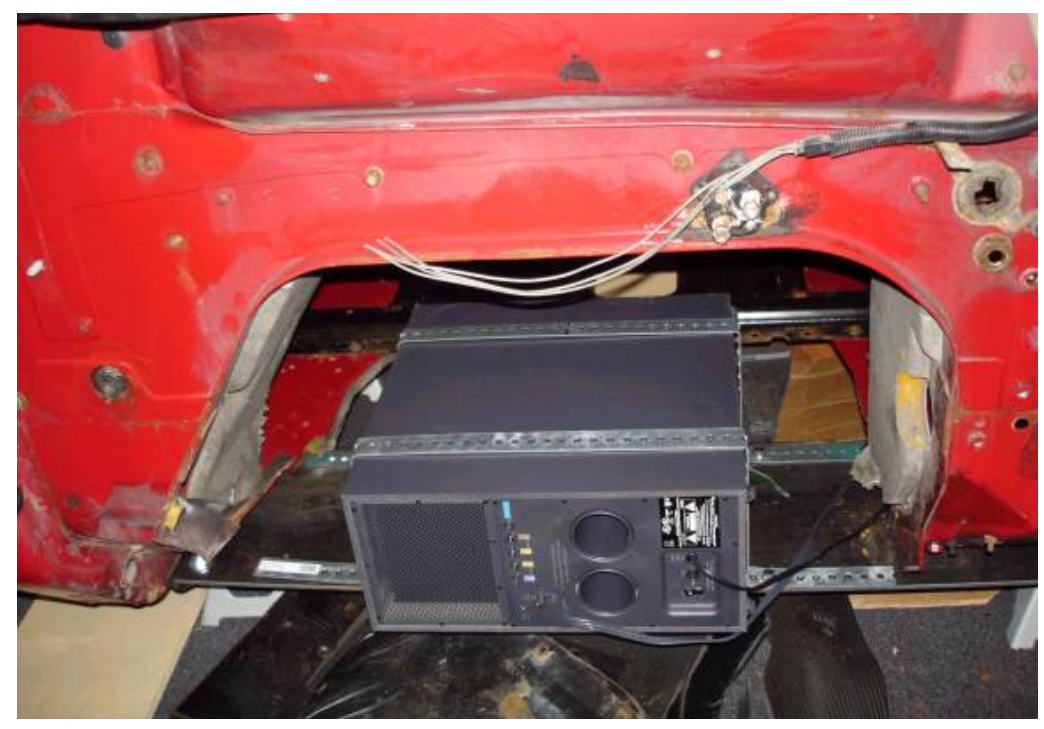

Figure B.17: Subwoofer in place of the engine

\section{CREATION OF THE DRIVING SCENARIO}

The research team at CISR has developed a driving scenario representing a monotonous driving environment that is likely to induce drivers' boredom and drowsiness. This scenario includes realistic sounds, visual effects, and other pre-defined events regarding road traffic and traffic controls. The driving scenario included both the geometric data for a section of I-70 and the highway signs and other 3 dimensional objects on this section of roadway.

\section{Interstate I-70 Geometric Data}

The road network selected for the driving scenario of the drowsiness experiments is based on a section of I-70 in Kansas. The research team acquired construction drawings from Kansas DOT and extracted the following alignment and marking data (complete geometric data is provided in Table). The codes in this table include:

\begin{tabular}{|c|c|c|}
\hline $\mathrm{BVC}$ & $=$ & Beginning of vertical curve \\
\hline PI & $=$ & Point of intersection \\
\hline EVC & $=$ & End of vertical section \\
\hline LVC & $=$ & Length of vertical Curve \\
\hline $\mathrm{BC}$ & $=$ & Beginning of curve \\
\hline $\mathrm{EC}$ & $=$ & End of curve \\
\hline$\Delta$ & $=$ & Deflection angle (degree, min, sec) \\
\hline $\operatorname{Rad}$ & $=$ & Radius of horizontal curve (ft) \\
\hline
\end{tabular}




$\begin{array}{lll}\text { Tan } & = & \text { Length of tangent }(\mathrm{ft}) \\ \mathrm{LN} & = & \text { Length of horizontal curve }(\mathrm{ft}) \\ \mathrm{R} & = & \text { Right hand curve } \\ \mathrm{L} & = & \text { Left hand curve }\end{array}$

The roadway marking diagram is shown in Figure B.18. Other data for this roadway includes the following:

\begin{tabular}{|c|c|c|}
\hline Number of lanes & $=$ & Two in each direction \\
\hline Lane width & $=$ & $3.6 \mathrm{~m}(12 \mathrm{ft})$ \\
\hline Right Shoulder width & $=$ & $3 \mathrm{~m}$ \\
\hline
\end{tabular}

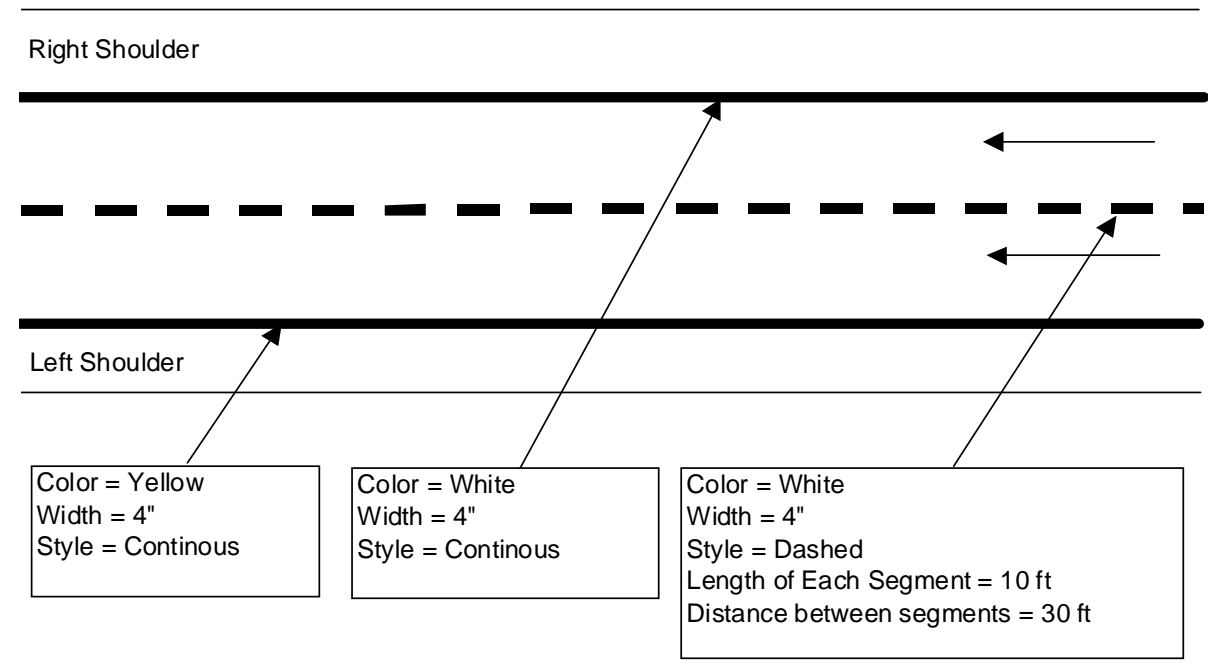

Figure B.18: Roadway marking

A three-dimensional road geometry was created based on this geometric data. Figure B.19 shows the original geometric data on the left, a view of the modeled roadway terrain in the middle, and an overview of the model of the entire terrain on the right.
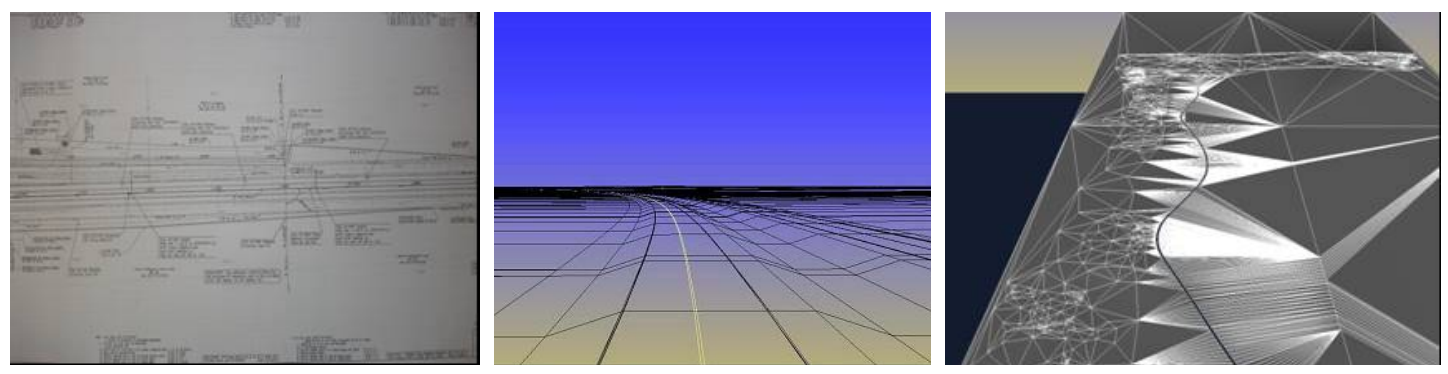

Figure B.19: I-70 cross section drawings and corresponding simulation geometry created using SGI OpenGL Performer 


\section{Highway Signs and Other Roadway Scenery}

To complete the driving environment a variety of 3 dimensional objects were designed and placed on the roadway geometry, including:

- $\quad$ Roadside objects, such as villages, trees, bushes, shown in Figure B.20

- Traffic signs, shown in Figure B.21

- The driving scene background, shown in Figure B.22

- Typical U.S. vehicles were created by INRETS based on pictures provided by CISR and integrated to the driving scenario, shown in Figures B.23 and B.24
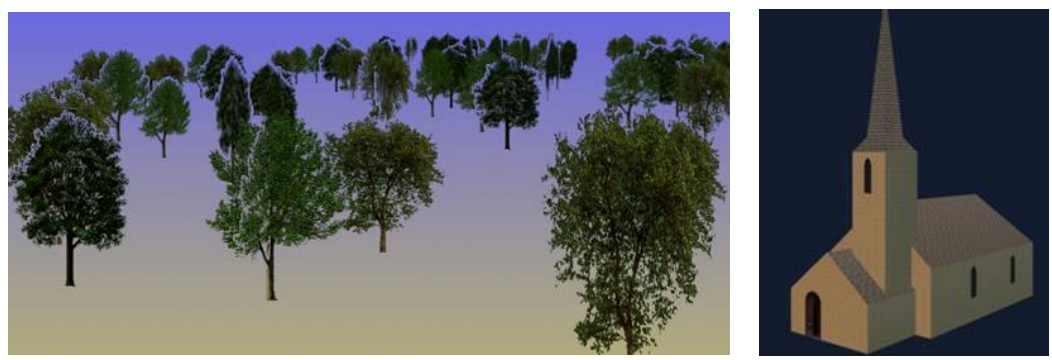

Figure B.20: 3D roadside elements included in the driving scenario
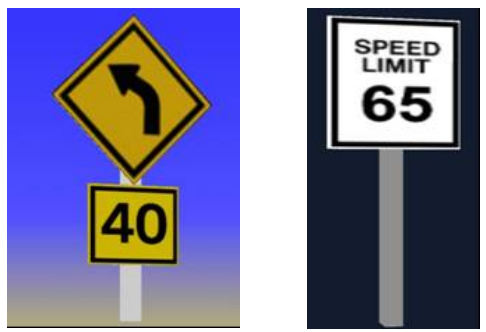

Figure B.21: Traffic signs placed on the roadway network

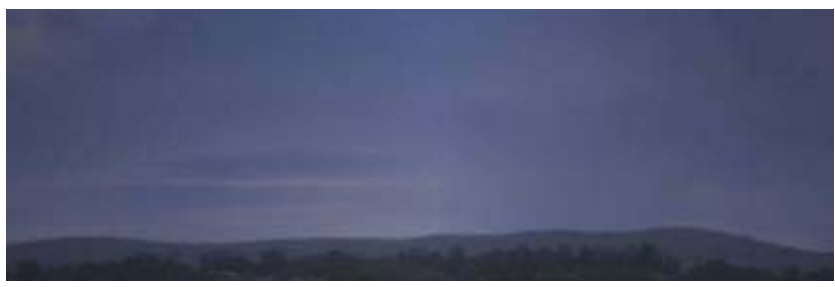

Figure B.22: Driving scene background 

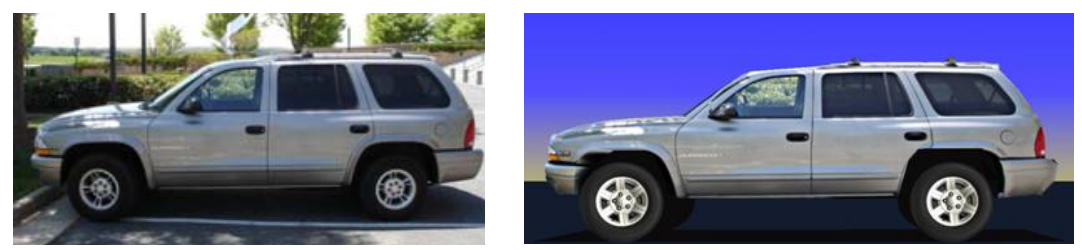

Figure B.23: Picture and corresponding 3D model of a Dodge Durango
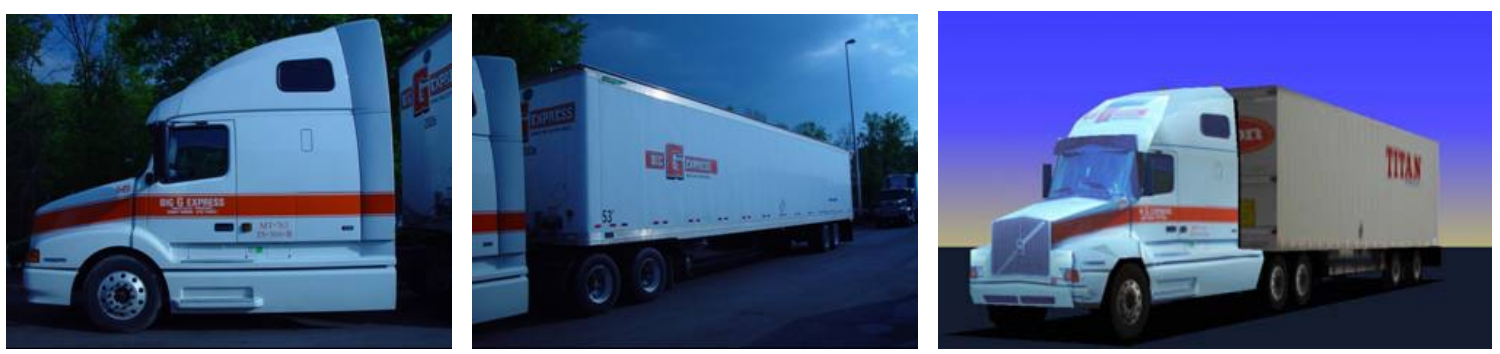

Figure B.24: Pictures and corresponding 3D model of a Century tractor semi-trailer 
Table B.1: Geometric design data for selected portion of I-70

\begin{tabular}{|c|c|c|c|c|c|c|c|c|c|c|c|c|c|c|}
\hline \multicolumn{2}{|c|}{ STATION } & \multicolumn{5}{|c|}{ VERTICAL CURVE } & \multicolumn{8}{|c|}{ HORIZONTAL CURVE } \\
\hline FT & $\underline{\text { Mile }}$ & GR(\%) & $\underline{\text { BVC }}$ & $\underline{\mathbf{P I}}$ & $\underline{\text { EVC }}$ & $\underline{\operatorname{LVC}(\mathrm{ft})}$ & $\underline{\mathrm{BC}}$ & $\underline{\mathbf{P I}}$ & $\underline{E C}$ & $\Delta(\mathbf{D M S})$ & Rad(ft) & $\underline{\operatorname{Tan}(f t)}$ & $\underline{\mathbf{L N}}$ (ft) & $\underline{\text { Dir }}$ \\
\hline Sec 1 & & & & & & & & & & & & & & \\
\hline 0 & 0.000 & -0.05 & & & & & & & & & & & & \\
\hline 1070 & 0.203 & 0.89 & 1070 & 1220 & 1370 & 300 & & & & & & & & \\
\hline 3500 & 0.663 & -2.13 & 3500 & 4350 & 5200 & 1700 & & & & & & & & \\
\hline 5407 & 1.024 & & & & & & 5407 & 5770 & 6128 & $14,25,46$ & 2864.79 & 362.66 & 721 & $\mathrm{R}$ \\
\hline 5290 & 1.002 & -0.04 & 5290 & 5640 & 5990 & 700 & & & & & & & & \\
\hline 11044 & 2.092 & & & & & & 11044 & 11898 & 12703.7 & $33,11,36$ & 2864.79 & 853.85 & 1659.7 & $\mathrm{~L}$ \\
\hline 12077 & 2.287 & 0.62 & 12077 & 12377 & 12677 & 600 & & & & & & & & \\
\hline 14204 & 2.690 & 3.05 & 14204 & 14655 & 15104 & 900 & & & & & & & & \\
\hline 15875 & 3.007 & -2.54 & 15875 & 16915 & 17955 & 2080 & & & & & & & & \\
\hline 17515 & 3.317 & & & & & & 17515 & 18174 & 18829.24 & $5,15,25$ & 14323.9 & 657.6 & 1314.24 & $\mathrm{R}$ \\
\hline 18182 & 3.444 & -0.45 & 18182 & 18532 & 18882 & 700 & & & & & & & & \\
\hline
\end{tabular}




\begin{tabular}{|c|c|c|c|c|c|c|c|c|c|c|c|c|c|c|}
\hline \multicolumn{2}{|c|}{ STATION } & \multicolumn{5}{|c|}{ VERTICAL CURVE } & \multicolumn{8}{|c|}{ HORIZONTAL CURVE } \\
\hline FT & $\underline{\text { Mile }}$ & $\underline{\text { GR(\%) }}$ & $\underline{\text { BVC }}$ & $\underline{\mathbf{P I}}$ & $\underline{\text { EVC }}$ & $\underline{\operatorname{LVC}(\mathrm{ft})}$ & $\underline{\mathrm{BC}}$ & $\underline{\mathbf{P I}}$ & $\underline{\mathbf{E C}}$ & $\underline{\Delta(\mathrm{DMS})}$ & $\operatorname{Rad}(f t)$ & $\underline{\operatorname{Tan}(\mathbf{f t})}$ & $\underline{\underline{L N}}(\mathbf{f t})$ & $\underline{\text { Dir }}$ \\
\hline 19609 & 3.714 & & & & & & 19609 & 21624 & 23615 & $14,41,22$ & 15626 & 2014 & 4006 & $\mathrm{R}$ \\
\hline 21990 & 4.165 & -0.56 & 21990 & 22137 & 22290 & 300 & & & & & & & & \\
\hline 23300 & 4.413 & & & & & & & & & & & & & \\
\hline $\operatorname{Sec} 2$ & & & & & & & & & & & & & & \\
\hline 23600 & 4.470 & & & & & & 23600 & 25244.25 & 26888.5 & 22,05 & 8526.86 & 1664.94 & 3288.5 & $\mathrm{R}$ \\
\hline 25300 & 4.792 & -1.2 & 25300 & 25700 & 26100 & 800 & & & & & & & & \\
\hline 26750 & 5.066 & -0.3 & 26750 & 27050 & 27350 & 600 & & & & & & & & \\
\hline 30700 & 5.814 & 0.3 & 30700 & 31000 & 31300 & 600 & & & & & & & & \\
\hline 31600 & 5.985 & 4 & 31600 & 32000 & 32400 & 800 & & & & & & & & \\
\hline 32129 & 6.085 & & & & & & 32129 & 34573.4 & 37017.8 & $45,31,56$ & 5771.58 & 2601.86 & 4888.8 & $\mathrm{~L}$ \\
\hline 33850 & 6.411 & -3.27 & 33850 & 35200 & 36550 & 2700 & & & & & & & & \\
\hline 38350 & 7.263 & -0.24 & 38350 & 38700 & 39050 & 700 & & & & & & & & \\
\hline 41100 & 7.784 & 2.05 & 41100 & 41700 & 42300 & 1200 & & & & & & & & \\
\hline
\end{tabular}




\begin{tabular}{|c|c|c|c|c|c|c|c|c|c|c|c|c|c|c|}
\hline \multicolumn{2}{|c|}{ STATION } & \multicolumn{5}{|c|}{ VERTICAL CURVE } & \multicolumn{8}{|c|}{ HORIZONTAL CURVE } \\
\hline FT & $\underline{\text { Mile }}$ & $\underline{\text { GR(\%) }}$ & $\underline{\text { BVC }}$ & $\underline{\mathbf{P I}}$ & $\underline{\text { EVC }}$ & $\underline{\operatorname{LVC}(\mathrm{ft})}$ & $\underline{\mathrm{BC}}$ & $\underline{\mathbf{P I}}$ & $\underline{\text { EC }}$ & $\underline{\Delta(\mathrm{DMS})}$ & $\operatorname{Rad}(f t)$ & $\underline{\operatorname{Tan}(\mathbf{f t})}$ & $\underline{\mathbf{L N}}(\mathbf{f t})$ & $\underline{\text { Dir }}$ \\
\hline 42700 & 8.087 & 3.98 & 42700 & 43000 & 43300 & 600 & & & & & & & & \\
\hline 46450 & 8.797 & 0.25 & 46450 & 47200 & 47950 & 1500 & & & & & & & & \\
\hline 48600 & 9.205 & 3.92 & 48600 & 49000 & 49400 & 800 & & & & & & & & \\
\hline 49850 & 9.441 & -0.25 & 49850 & 50850 & 51850 & 2000 & & & & & & & & \\
\hline 50399 & 9.545 & & & & & & 50399 & 51647.4 & 52895.8 & $4,09,41$ & 34377.47 & 1248.97 & 2496.8 & $\mathrm{~L}$ \\
\hline 52350 & 9.915 & -1.66 & 52350 & 52750 & 53150 & 800 & & & & & & & & \\
\hline 55550 & 10.521 & -3.47 & 55550 & 56000 & 56450 & 900 & & & & & & & & \\
\hline 60200 & 11.402 & -0.83 & 60200 & 60700 & 61200 & 1000 & & & & & & & & \\
\hline 62300 & 11.799 & 0 & 62300 & 62600 & 62900 & 600 & & & & & & & & \\
\hline 63377 & 12.003 & 3.84 & 63377 & 63839.5 & 64302 & 925 & & & & & & & & \\
\hline 64400 & 12.197 & & & & & & & & & & & & & \\
\hline $\operatorname{Sec} 3$ & & & & & & & & & & & & & & \\
\hline 65729 & 12.449 & -1 & 65729 & 66454 & 67179 & 1450 & & & & & & & & \\
\hline
\end{tabular}




\begin{tabular}{|c|c|c|c|c|c|c|c|c|c|c|c|c|c|c|}
\hline \multicolumn{2}{|c|}{ STATION } & \multicolumn{5}{|c|}{ VERTICAL CURVE } & \multicolumn{8}{|c|}{ HORIZONTAL CURVE } \\
\hline FT & $\underline{\text { Mile }}$ & GR(\%) & $\underline{\text { BVC }}$ & $\underline{\mathbf{P I}}$ & $\underline{\text { EVC }}$ & $\underline{\operatorname{LVC}(\mathbf{f t})}$ & $\underline{\mathrm{BC}}$ & $\underline{\mathbf{P I}}$ & $\underline{\mathrm{EC}}$ & $\Delta(\mathbf{D M S})$ & Rad(ft) & $\underline{\operatorname{Tan}(f t)}$ & $\underline{\mathbf{L N}}(\mathbf{f t})$ & $\underline{\text { Dir }}$ \\
\hline 66512 & 12.597 & & & & & & 66512 & 67278.74 & 68045.48 & $11,33,54$ & 7597 & 769.35 & 1533.48 & $\mathrm{~L}$ \\
\hline 68004 & 12.880 & 0.24 & 68004 & 68304 & 68604 & 600 & & & & & & & & \\
\hline 71204 & 13.486 & 2.76 & 71204 & 71504 & 71804 & 600 & & & & & & & & \\
\hline 71663 & 13.573 & & & & & & 71663 & 72730 & 73797 & $10,37,45$ & 11503.9 & 1070 & 2134 & $\mathrm{R}$ \\
\hline 73829 & 13.983 & 0.84 & 73829 & 74129 & 74429 & 600 & & & & & & & & \\
\hline 75904 & 14.376 & 2.44 & 75904 & 76204 & 76504 & 600 & & & & & & & & \\
\hline 79861 & 15.125 & & & & & & 79861 & 81383.5 & 82906 & $10,07,36$ & 17231 & 1526 & 3045 & $\mathrm{~L}$ \\
\hline 80629 & 15.271 & -3.56 & 80629 & 81504 & 82379 & 1750 & & & & & & & & \\
\hline 83829 & 15.877 & -0.2 & 83829 & 84129 & 84429 & 600 & & & & & & & & \\
\hline 85204 & 16.137 & 0.24 & 85204 & 85354 & 85504 & 300 & & & & & & & & \\
\hline 87204 & 16.516 & 3.04 & 87204 & 87504 & 87804 & 600 & & & & & & & & \\
\hline 89229 & 16.899 & 0.56 & 89229 & 89604 & 89979 & 750 & & & & & & & & \\
\hline 89914 & 17.029 & & & & & & 89914 & 91085 & 92256 & $7,49,45$ & 17145 & 1173.2 & 2342 & $\mathrm{R}$ \\
\hline
\end{tabular}




\begin{tabular}{|c|c|c|c|c|c|c|c|c|c|c|c|c|c|c|}
\hline \multicolumn{2}{|c|}{ STATION } & \multicolumn{5}{|c|}{ VERTICAL CURVE } & \multicolumn{8}{|c|}{ HORIZONTAL CURVE } \\
\hline FT & $\underline{\text { Mile }}$ & $\underline{\text { GR(\%) }}$ & $\underline{\text { BVC }}$ & $\underline{\mathbf{P I}}$ & $\underline{\text { EVC }}$ & $\underline{\operatorname{LVC}(\mathrm{ft})}$ & $\underline{\mathrm{BC}}$ & $\underline{\mathbf{P I}}$ & $\underline{\mathrm{EC}}$ & $\underline{\Delta(\mathrm{DMS})}$ & $\operatorname{Rad}(f t)$ & $\underline{\operatorname{Tan}(f t)}$ & $\underline{\underline{L N}}(\mathbf{f t})$ & $\underline{\text { Dir }}$ \\
\hline 91814 & 17.389 & 2.92 & 91814 & 92114 & 92414 & 600 & & & & & & & & \\
\hline 93014 & 17.616 & 0 & 93014 & 93564 & 94114 & 1100 & & & & & & & & \\
\hline 94604 & 17.917 & -2.16 & 94604 & 95004 & 95404 & 800 & & & & & & & & \\
\hline 98060 & 18.572 & & & & & & 98060 & 99586.5 & 101113 & $15,19,32$ & 11414 & 1535 & 3053 & $\mathrm{R}$ \\
\hline 99079 & 18.765 & 1 & 99079 & 99379 & 99679 & 600 & & & & & & & & \\
\hline 100454 & 19.025 & -0.68 & 100454 & 100804 & 101154 & 700 & & & & & & & & \\
\hline 101834 & 19.287 & -3.96 & 101834 & 102434 & 103034 & 1200 & & & & & & & & \\
\hline 105171 & 19.919 & & & & & & & & & & & & & \\
\hline $\operatorname{Sec} 4$ & & & & & & & & & & & & & & \\
\hline 105469 & 19.975 & -1.98 & 105469 & 105794 & 106119 & 650 & & & & & & & & \\
\hline 106971 & 20.260 & -3.84 & 106971 & 107361 & 107751 & 780 & & & & & & & & \\
\hline 108841 & 20.614 & 1.81 & 108841 & 109266 & 109691 & 850 & & & & & & & & \\
\hline 109991 & 20.832 & -4 & 109991 & 111071 & 112151 & 2160 & & & & & & & & \\
\hline
\end{tabular}




\begin{tabular}{|c|c|c|c|c|c|c|c|c|c|c|c|c|c|c|}
\hline \multicolumn{2}{|c|}{ STATION } & \multicolumn{5}{|c|}{ VERTICAL CURVE } & \multicolumn{8}{|c|}{ HORIZONTAL CURVE } \\
\hline FT & $\underline{\text { Mile }}$ & $\underline{\text { GR(\%) }}$ & $\underline{\text { BVC }}$ & $\underline{\mathbf{P I}}$ & $\underline{\text { EVC }}$ & $\underline{\operatorname{LVC}(\mathrm{ft})}$ & $\underline{\mathrm{BC}}$ & $\underline{\mathbf{P I}}$ & $\underline{\text { EC }}$ & $\underline{\Delta(\mathrm{DMS})}$ & $\operatorname{Rad}(f t)$ & $\underline{\operatorname{Tan}(f t)}$ & $\underline{\underline{L N}}(\mathbf{f t})$ & $\underline{\text { Dir }}$ \\
\hline 113371 & 21.472 & 0.28 & 113371 & 113731 & 114091 & 720 & & & & & & & & \\
\hline 114321 & 21.652 & 0.12 & 114321 & 114485 & 114649 & 328 & & & & & & & & \\
\hline 116421 & 22.049 & 0 & 116421 & 116583 & 116746 & 325 & & & & & & & & \\
\hline 118199 & 22.386 & 1.88 & 118199 & 118494 & 118789 & 590 & & & & & & & & \\
\hline 119511 & 22.635 & 0.29 & 119511 & 119901 & 120291 & 780 & & & & & & & & \\
\hline 121891 & 23.085 & 2.07 & 121891 & 122151 & 122411 & 520 & & & & & & & & \\
\hline 125221 & 23.716 & -3.57 & 125221 & 126271 & 127321 & 2100 & & & & & & & & \\
\hline 128271 & 24.294 & -2.268 & 128271 & 128466 & 128661 & 390 & & & & & & & & \\
\hline 130671 & 24.748 & 0.542 & 130671 & 130931 & 131191 & 520 & & & & & & & & \\
\hline 132165 & 25.031 & & & & & & & & & & & & & \\
\hline $\operatorname{Sec} 5$ & & & & & & & & & & & & & & \\
\hline 132834 & 25.158 & & & & & & 132834.3 & 132994.3 & 133154.2 & $1,7,4.3$ & 16404.1 & 160.0 & 319.9 & $\mathrm{~L}$ \\
\hline 133743 & 25.330 & -2.969 & 133743 & 134393 & 135043 & 1300 & & & & & & & & \\
\hline
\end{tabular}




\begin{tabular}{|c|c|c|c|c|c|c|c|c|c|c|c|c|c|c|}
\hline \multicolumn{2}{|c|}{ STATION } & \multicolumn{5}{|c|}{ VERTICAL CURVE } & \multicolumn{8}{|c|}{ HORIZONTAL CURVE } \\
\hline FT & $\underline{\text { Mile }}$ & $\underline{\text { GR(\%) }}$ & $\underline{\text { BVC }}$ & $\underline{\mathbf{P I}}$ & $\underline{\text { EVC }}$ & $\underline{\operatorname{LVC}(\mathrm{ft})}$ & $\underline{\mathrm{BC}}$ & $\underline{\mathbf{P I}}$ & $\underline{\text { EC }}$ & $\underline{\Delta(\mathrm{DMS})}$ & $\operatorname{Rad}(f t)$ & $\underline{\operatorname{Tan}(f t)}$ & $\underline{\underline{L N}}(\mathbf{f t})$ & $\underline{\text { Dir }}$ \\
\hline 136000 & 25.758 & -3.561 & 136000 & 136195 & 136390 & 390 & & & & & & & & \\
\hline 137840 & 26.106 & -1.493 & 137840 & 138035 & 138230 & 390 & & & & & & & & \\
\hline 139305 & 26.384 & 0.977 & 139305 & 139492 & 139680 & 375 & & & & & & & & \\
\hline 140990 & 26.703 & 1.75 & 140990 & 141615 & 142240 & 1250 & & & & & & & & \\
\hline 142730 & 27.032 & 0.356 & 142730 & 142925 & 143120 & 390 & & & & & & & & \\
\hline 144435 & 27.355 & -2.004 & 144435 & 144960 & 145485 & 1050 & & & & & & & & \\
\hline 146167 & 27.683 & & & & & & 146167.6 & 147228.3 & 148289 & $1,24,54$ & 26182 & 1059.7 & 2121.3 & $\mathrm{R}$ \\
\hline 146255 & 27.700 & -1.158 & 146255 & 146450 & 146645 & 390 & & & & & & & & \\
\hline 147255 & 27.889 & -1.92 & 147255 & 147450 & 147645 & 390 & & & & & & & & \\
\hline 148115 & 28.052 & 0.32 & 148115 & 148632 & 149150 & 1035 & & & & & & & & \\
\hline 149980 & 28.405 & -0.24 & 149980 & 150275 & 150570 & 590 & & & & & & & & \\
\hline 153275 & 29.029 & -0.712 & 153275 & 153570 & 153865 & 590 & & & & & & & & \\
\hline 155145 & 29.384 & -0.235 & 155145 & 155440 & 155735 & 590 & & & & & & & & \\
\hline
\end{tabular}




\begin{tabular}{|c|c|c|c|c|c|c|c|c|c|c|c|c|c|c|}
\hline \multicolumn{2}{|c|}{ STATION } & \multicolumn{5}{|c|}{ VERTICAL CURVE } & \multicolumn{8}{|c|}{ HORIZONTAL CURVE } \\
\hline FT & $\underline{\text { Mile }}$ & $\underline{\text { GR(\%) }}$ & $\underline{\text { BVC }}$ & $\underline{\mathbf{P I}}$ & $\underline{\text { EVC }}$ & $\underline{\operatorname{LVC}(\mathrm{ft})}$ & $\underline{\mathrm{BC}}$ & $\underline{\mathbf{P I}}$ & $\underline{\mathrm{EC}}$ & $\underline{\Delta(\mathrm{DMS})}$ & $\operatorname{Rad}(f t)$ & $\underline{\operatorname{Tan}(f t)}$ & $\underline{\underline{L N}}(\mathbf{f t})$ & $\underline{\text { Dir }}$ \\
\hline 157475 & 29.825 & 0 & 157475 & 157750 & 158025 & 550 & & & & & & & & \\
\hline 160452 & 30.389 & & & & & & & & & & & & & \\
\hline $\operatorname{Sec} 6$ & & & & & & & & & & & & & & \\
\hline 160727 & 30.441 & & & & & & 160727.6 & 162026.3 & 163325 & $25,47,08$ & 5770.9 & 1320.8 & 2597.4 & $\mathrm{~L}$ \\
\hline 161977 & 30.677 & 2.608 & 161977 & 162417 & 162857 & 880 & & & & & & & & \\
\hline 162892 & 30.851 & 0.436 & 162892 & 163217 & 163542 & 650 & & & & & & & & \\
\hline 163582 & 30.981 & -1.52 & 163582 & 163857 & 164132 & 550 & & & & & & & & \\
\hline 164602 & 31.175 & 3.88 & 164602 & 164902 & 165202 & 600 & & & & & & & & \\
\hline 167217 & 31.670 & & & & & & 167217.1 & 168470.3 & 169723.5 & $25,14,56$ & 5687.6 & 1273.8 & 2506.3 & $\mathrm{R}$ \\
\hline 169566 & 32.115 & -1.108 & 169566 & 169891 & 170216 & 650 & & & & & & & & \\
\hline 171052 & 32.396 & -1.203 & 171052 & 171217 & 171382 & 330 & & & & & & & & \\
\hline 172352 & 32.642 & -0.503 & 172352 & 172677 & 173002 & 650 & & & & & & & & \\
\hline 174177 & 32.988 & 1.278 & 174177 & 174677 & 175177 & 1000 & & & & & & & & \\
\hline
\end{tabular}




\begin{tabular}{|c|c|c|c|c|c|c|c|c|c|c|c|c|c|c|}
\hline \multicolumn{2}{|c|}{ STATION } & \multicolumn{5}{|c|}{ VERTICAL CURVE } & \multicolumn{8}{|c|}{ HORIZONTAL CURVE } \\
\hline FT & $\underline{\text { Mile }}$ & $\underline{\mathrm{GR}(\%)}$ & $\underline{\text { BVC }}$ & $\underline{\mathbf{P I}}$ & $\underline{\text { EVC }}$ & $\underline{\operatorname{LVC}(\mathrm{ft})}$ & $\underline{\mathrm{BC}}$ & $\underline{\mathbf{P I}}$ & $\underline{\mathrm{EC}}$ & $\underline{\Delta(\mathrm{DMS})}$ & $\operatorname{Rad}(f t)$ & $\underline{\operatorname{Tan}(f t)}$ & $\underline{\underline{L N}}(\mathbf{f t})$ & $\underline{\text { Dir }}$ \\
\hline 175152 & 33.173 & 1.739 & 175152 & 175447 & 175742 & 590 & & & & & & & & \\
\hline 176052 & 33.343 & 0.312 & 176052 & 176282 & 176512 & 460 & & & & & & & & \\
\hline 177784 & 33.671 & & & & & & 177784.7 & 178250.6 & 178716.4 & $1,33,16$ & 34333.9 & 465.7 & 931.7 & $\mathrm{R}$ \\
\hline 174682 & 33.084 & 4 & 174682 & 175142 & 175602 & 920 & & & & & & & & \\
\hline 180052 & 34.101 & -4.7 & 180052 & 181262 & 182472 & 2420 & & & & & & & & \\
\hline 180717 & 34.227 & & & & & & 180717.7 & 181193.5 & 181669.2 & $0,57,14$ & 5733.5 & 477.29 & 951.44 & $\mathrm{R}$ \\
\hline 183352 & 34.726 & -0.185 & 183352 & 183812 & 184272 & 920 & & & & & & & & \\
\hline 185302 & 35.095 & -1.08 & 185302 & 185597 & 185892 & 590 & & & & & & & & \\
\hline 188402 & 35.682 & 2.78 & 188402 & 188892 & 189382 & 980 & & & & & & & & \\
\hline 189402 & 35.872 & 0.364 & 189402 & 190052 & 190702 & 1300 & & & & & & & & \\
\hline 191152 & 36.203 & 2.775 & 191152 & 191802 & 192452 & 1300 & & & & & & & & \\
\hline 195517 & 37.030 & & & & & & 195517.6 & 195906.1 & 196294.5 & $0,43,39$ & 61186.6 & 388.46 & 776.90 & $\mathrm{~L}$ \\
\hline 194702 & 36.875 & -3.44 & 194702 & 195767 & 196832 & 2130 & & & & & & & & \\
\hline
\end{tabular}




\begin{tabular}{|c|c|c|c|c|c|c|c|c|c|c|c|c|c|c|}
\hline \multicolumn{2}{|c|}{ STATION } & \multicolumn{5}{|c|}{ VERTICAL CURVE } & \multicolumn{8}{|c|}{ HORIZONTAL CURVE } \\
\hline FT & $\underline{\text { Mile }}$ & $\underline{\text { GR(\%) }}$ & $\underline{\text { BVC }}$ & $\underline{\mathbf{P I}}$ & $\underline{\text { EVC }}$ & $\underline{\operatorname{LVC}(\mathrm{ft})}$ & $\underline{\mathrm{BC}}$ & $\underline{\mathbf{P I}}$ & $\underline{\text { EC }}$ & $\underline{\Delta(\mathrm{DMS})}$ & $\operatorname{Rad}(f t)$ & $\underline{\operatorname{Tan}(f t)}$ & $\underline{\mathbf{L N}}(\mathbf{f t})$ & $\underline{\text { Dir }}$ \\
\hline 199752 & 37.832 & 4.108 & 199752 & 200472 & 201192 & 1440 & & & & & & & & \\
\hline 201202 & 38.106 & -4.487 & 201202 & 202677 & 204152 & 2950 & & & & & & & & \\
\hline 204177 & 38.670 & 1.38 & 204177 & 204734 & 205292 & 1115 & & & & & & & & \\
\hline 206002 & 39.016 & -2.35 & 206002 & 206652 & 207302 & 1300 & & & & & & & & \\
\hline 207623 & 39.323 & & & & & & & & & & & & & \\
\hline Sec 7 & & & & & & & & & & & & & & \\
\hline 209023 & 39.588 & 4.39 & 1400 & 2250 & 3100 & 1700 & & & & & & & & \\
\hline 210973 & 39.957 & -1.459 & 3350 & 4325 & 5300 & 1950 & & & & & & & & \\
\hline 211190 & 39.998 & & & & & & 211190.2 & 211731.5 & 212272.9 & $2,42,26$ & 22916.6 & 541.33 & 1082.67 & $\mathrm{~L}$ \\
\hline 212984 & 40.338 & & & & & & 212984.8 & 213660.6 & 214336.5 & $2,42,35$ & 28608.9 & 676.50 & 1351.70 & $\mathrm{R}$ \\
\hline 213423 & 40.421 & -0.354 & 213423 & 213536 & 213648.9 & 225 & & & & & & & & \\
\hline 215673 & 40.847 & 0.754 & 215673 & 215836 & 215998.9 & 325 & & & & & & & & \\
\hline 217623 & 41.217 & 3.26 & 217623 & 217948 & 218273.9 & 650 & & & & & & & & \\
\hline
\end{tabular}




\begin{tabular}{|c|c|c|c|c|c|c|c|c|c|c|c|c|c|c|}
\hline \multicolumn{2}{|c|}{ STATION } & \multicolumn{5}{|c|}{ VERTICAL CURVE } & \multicolumn{8}{|c|}{ HORIZONTAL CURVE } \\
\hline FT & $\underline{\text { Mile }}$ & $\underline{\mathrm{GR}(\%)}$ & $\underline{\text { BVC }}$ & $\underline{\mathbf{P I}}$ & $\underline{\text { EVC }}$ & $\underline{\operatorname{LVC}(\mathrm{ft})}$ & $\underline{\mathrm{BC}}$ & $\underline{\mathbf{P I}}$ & $\underline{\text { EC }}$ & $\underline{\Delta(\mathrm{DMS})}$ & $\operatorname{Rad}(f t)$ & $\underline{\operatorname{Tan}(f t)}$ & $\underline{\underline{L N}}(\mathbf{f t})$ & $\underline{\text { Dir }}$ \\
\hline 218773 & 41.434 & -1.69 & 218773 & 219973 & 221173.9 & 2400 & & & & & & & & \\
\hline 221223 & 41.898 & -0.612 & 221223 & 221373 & 221523.9 & 300 & & & & & & & & \\
\hline 223723 & 42.372 & 0.469 & 223723 & 223923 & 224123.9 & 400 & & & & & & & & \\
\hline 225223 & 42.656 & -3.046 & 225223 & 226098 & 226973.9 & 1750 & & & & & & & & \\
\hline 227873 & 43.158 & 1.575 & 227873 & 228436 & 228998.9 & 1125 & & & & & & & & \\
\hline 229228 & 43.414 & & & & & & & & & & & & & \\
\hline 229228 & 43.414 & & & & & & & & & & & & & \\
\hline 231078 & 43.765 & 0.598 & 231078 & 231328 & 231578 & 500 & & & & & & & & \\
\hline 235278 & 44.560 & -0.683 & 235278 & 235603 & 235928 & 650 & & & & & & & & \\
\hline 236878 & 44.863 & -2.016 & 236878 & 237340 & 237803 & 925 & & & & & & & & \\
\hline 237020 & 44.890 & & & & & & 237020 & 237233.2 & 237446.5 & $0,25,34$ & 57332.6 & 212.92 & 426.50 & $\mathrm{~L}$ \\
\hline 241528 & 45.744 & 2.023 & 241528 & 241965 & 242403 & 875 & & & & & & & & \\
\hline 246728 & 46.729 & -1.535 & 246728 & 247603 & 248478 & 1750 & & & & & & & & \\
\hline
\end{tabular}




\begin{tabular}{|c|c|c|c|c|c|c|c|c|c|c|c|c|c|c|}
\hline \multicolumn{2}{|c|}{ STATION } & \multicolumn{5}{|c|}{ VERTICAL CURVE } & \multicolumn{8}{|c|}{ HORIZONTAL CURVE } \\
\hline FT & $\underline{\text { Mile }}$ & $\underline{\text { GR(\%) }}$ & $\underline{\text { BVC }}$ & $\underline{\mathbf{P I}}$ & $\underline{\text { EVC }}$ & $\underline{\operatorname{LVC}(\mathrm{ft})}$ & $\underline{\mathrm{BC}}$ & $\underline{\mathbf{P I}}$ & $\underline{\mathrm{EC}}$ & $\underline{\Delta(\mathrm{DMS})}$ & $\operatorname{Rad}(f t)$ & $\underline{\operatorname{Tan}(f t)}$ & $\underline{\underline{L N}}(\mathbf{f t})$ & $\underline{\text { Dir }}$ \\
\hline 247380 & 46.852 & & & & & & 247380.9 & 247661.4 & 247941.9 & $2,47,40$ & 11496 & 280.44 & 561.02 & $\mathrm{~L}$ \\
\hline 248834 & 47.128 & & & & & & 248834.3 & 249280.5 & 249726.7 & $4,28,52$ & 11417.3 & 446.19 & 892.38 & $\mathrm{R}$ \\
\hline 249678 & 47.288 & -2.873 & 249678 & 250103 & 250528 & 850 & & & & & & & & \\
\hline 251429 & 47.619 & & & & & & & & & & & & & \\
\hline $\operatorname{Sec} 8$ & & & & & & & & & & & & & & \\
\hline 252879 & 47.894 & 1.04 & 252879 & 253179 & 253479 & 600 & & & & & & & & \\
\hline 254729 & 48.244 & 0 & 254729 & 255029 & 255329 & 600 & & & & & & & & \\
\hline 255929 & 48.471 & 0.68 & 255929 & 256229 & 256529 & 600 & & & & & & & & \\
\hline 258157 & 48.893 & & & & & & 258157 & 260462 & 262767 & $28,25,47$ & 9291 & 2353 & 4610 & $\mathrm{~L}$ \\
\hline 258779 & 49.011 & -1.36 & 258779 & 259379 & 259979 & 1200 & & & & & & & & \\
\hline 260464 & 49.330 & 3.2 & 260464 & 261094 & 261724 & 1260 & & & & & & & & \\
\hline 264242 & 50.046 & & & & & & 264242.6 & 265280.1 & 266317.6 & $20,45,09$ & 5729 & 1049 & 2075 & $\mathrm{R}$ \\
\hline 264529 & 50.100 & -1.04 & 264529 & 265529 & 266529 & 2000 & & & & & & & & \\
\hline
\end{tabular}




\begin{tabular}{|c|c|c|c|c|c|c|c|c|c|c|c|c|c|c|}
\hline \multicolumn{2}{|c|}{ STATION } & \multicolumn{5}{|c|}{ VERTICAL CURVE } & \multicolumn{8}{|c|}{ HORIZONTAL CURVE } \\
\hline FT & $\underline{\text { Mile }}$ & $\underline{\text { GR(\%) }}$ & $\underline{\text { BVC }}$ & $\underline{\mathbf{P I}}$ & $\underline{\text { EVC }}$ & $\underline{\operatorname{LVC}(\mathrm{ft})}$ & $\underline{\mathrm{BC}}$ & $\underline{\mathbf{P I}}$ & $\underline{\text { EC }}$ & $\underline{\Delta(\mathrm{DMS})}$ & $\operatorname{Rad}(f t)$ & $\underline{\operatorname{Tan}(\mathbf{f t})}$ & $\underline{\mathbf{L N}}(\mathbf{f t})$ & $\underline{\text { Dir }}$ \\
\hline 266879 & 50.545 & 2.16 & 266879 & 267229 & 267579 & 700 & & & & & & & & \\
\hline 268102 & 50.777 & & & & & & 268102 & 268510.5 & 268919 & $8,10,33$ & 5729 & 409 & 817 & $\mathrm{R}$ \\
\hline 268879 & 50.924 & -1.96 & 268879 & 269679 & 270479 & 1600 & & & & & & & & \\
\hline 270734 & 51.275 & & & & & & 270734 & 271306.9 & 271879.7 & $22,54,50$ & 2864 & 580 & 1145.7 & $\mathrm{R}$ \\
\hline 272265 & 51.565 & -1.68 & 272265 & 272465 & 272665 & 400 & & & & & & & & \\
\hline 272703 & 51.648 & & & & & & 272703 & 273176.5 & 273650 & $18,57,29$ & 2864 & 478 & 947 & $\mathrm{~L}$ \\
\hline 273429 & 51.786 & 1.36 & 273429 & 273779 & 274129 & 700 & & & & & & & & \\
\hline 274579 & 52.004 & -4.52 & 274579 & 275579 & 276579 & 2000 & & & & & & & & \\
\hline 276975 & 52.457 & & & & & & 276975 & 277517.5 & 278060 & $03,37,04$ & 17188 & 542 & 1085 & $\mathrm{~L}$ \\
\hline 277309 & 52.521 & -1.02 & 277309 & 277669 & 278029 & 720 & & & & & & & & \\
\hline 277758 & 52.606 & & & & & & & & & & & & & \\
\hline
\end{tabular}




\section{LIST OF REFERENCES}

Abe, G., and Richardson, J. (2004). "The Effect of Alarm Timing on Driver Behavior: An Investigation of Differences in Driver Trust and Response to Alarms According to Alarm Timing." Transportation Research Part F, 7, 307-322.

Akerstedt, T., and Gillberg, M. (1990). "Subjective and Objective Sleepiness in the Active Individual.” International Journal of Neuroscience, 52, 29-37.

Akerstedt, T., and Kecklund, G. (1994)., "Work Hours, Sleepiness and Accidents." Karolinska Institute (Stress Research Report No 248), Stockholm. Sweden.

Artaud, P., Planque, S., Lavergne, C., Cara, H., De Lepine, P., Tarriere, C., and Gueguen, C. "An On-board System for Detecting Lapses of Alertness in Car Driving." Proceedings of the 14th International Conference on Enhanced Safety of Vehicles, Munich, Germany.

Bekiaris, E. D., and Nikolaou, S. I. (2004). "Towards the Development of Design Guidelines Handbook for Driver Hypovigilance Detection and Warning - The AWAKE Approach.” Hellenic Institute of Transport, Greece.

Ben-Yaacov, A., Maltz, M., and Shinar, D. (2002). "Effects of an In-vehicle Collision Avoidance Warning System on Short-and Long-Term Driving Performance." Human Factors, 44(2), 335.

Bliss, J. P., and Acton, S. A. (2003). "Alarm Mistrust in Automobiles: How Collision Alarm Reliability Effects Driving.” Applied Ergonomics, (34), 499-509.

Braver, E., Preusser, C., Preusser, D., Baum, H., Beilock, R., and Ulmer, R. (1992). "Long Hours and Fatigue: A Study of Tractor-Trailer Drivers." Journal of Public Health Policy, 13(3), 341-366.

Brown, I. D. (1966). "Effects of Prolonged Driving Upon Driving Skills and Performance of a Subsidiary Task." Industrial Medicine and Surgery, 35, 760-765.

Brown, I. D. (1997). "Prospects for Technological Countermeasures Against Driver Fatigue." Accident Analysis and Prevention, 29.

Brown, T. L., Lee, J. D., and Hoffman, J. "The Effect of Rear-End Collision Warnings on On-Going Response." 45th Annual Meeting of the Human Factors and Ergonomics Society, Minneapolis, MN.

Campagne, A., Pebayle, T., and Muzet, A. (2004). "Correlation Between Driving Errors and Vigilance Level: Influence of the Driver Age." Physiology and Behavior, 80, 515-524.

Carskadon, M., and Dement, W. (1981). "Cumulative Effects of Sleep Restrictions on Daytime Sleepiness.” Psychology, 18, 107-118. 
Chaput, D., Petit, C., Planque, S., and Tarrière, C. (1990). "Un système embarqué de détection de l'hypovigilance." Journées d'études: le maintien de la vigilance dans les Transports. Lyon, France, INRETS, Lyon, France.

Chen, L., and Ulsoy, G. "Experimental validation of a robust steering assist controller on a driving simulator." American Control Conference, Anchorage, AK.

COMSIS Corporation. (1993a). "Human Factors Guidelines for Crash Avoidance Warning Devices.” NHTSA Contract No. DTNH22-91-C-07004, Washington, DC.

COMSIS Corporation. (1993b). "In-Vehicle Crash Avoidance Warnings: Major Research Needs." NHTSA Contract No. DTNH22-91-C-07004, Washington, DC.

Desmond, P. A., and Matthews, G. (1996). "Task-Induced Fatigue Effects on Simulated Driving Performance.” Vision in Vehicles VI, A. G. Gale, ed., North-Holland, Amsterdam.

Dinges, D. F. (1995). "An Overview of Sleepiness and Accidents." Journal of Sleep Research, 2 (supplement), 4-14.

Dingus, T. A., Hardee, L., and Wierwille, W. W. (1985). "Development of Impaired Driver Detection Measures." Department of Industrial Engineering and Operations Research, Virginia Polytechnic Institute and State University, (Departmental Report 8504), Blacksburg, VA.

Dingus, T. A., McGehee, D. V., Manakkal, N., Jahns, S. K., Carney, C., and Hankey, J. M. (1997). "Human Factors Field Evaluation of Automotive Headway Maintenance/Collision Warning Devices." Human Factors, 39(2), 216.

Dureman, E., and Boden, C. (1972). "Fatigue in Simulated Car Driving." Present Technological Status of Detecting Drowsy Driving Patterns. Jidosha Gijutsu, Y. Seko, ed., Central Research Institute, Nissan Motor Company, 547-554.

Edman, T. R. "Human Factors Guidelines for the Use of Synthetic Speech Devices." Proceedings of the Human Factors Society 26th Annual Meeting, Santa Monica, CA, 212-216.

Edworthy, J. (1994). "The Design and Implementation of Nonverbal Auditory Warnings.” Applied Ergonomics, 25(4), 202-210.

Elling, M., and Sherman, P. "Evaluation of Steering Wheel Measures for Drowsy Drivers." 27th ISATA, Aachen, Germany, 207-214.

Enriquez, M., and MacLean, K. E. "Impact of Haptic Warning Signal Reliability in a Time-and-Safety-Critical Task." IEEE 12th International Symposium on Haptic Interfaces for Virtual Environment and Teleoperator Systems (HAPTICS 2004), Chicago, IL. 
Erwin, C. W. (1976). "Studies of Drowsiness: Final Report." The National Driving Center, Durham, NC.

Espié, S., Mohellebi, H., and Kheddar, A. "A High Performance/Low-Cost Mini Driving Simulator Alternative for Human Factor Studies.” 2003 DSC North America, Dearborn, MI.

Fahey, S. E., and Wierwille, W. W. (1995). "Advisory and Alarm Stimuli Optimization for a Drowsy Driver Detection System." Semi-Annual Report, Research on Vehicle-Based Driver Status/Performance Monitoring: Seventh Semi-Annual Research Report, NHTSA, DOT HS 808 299, Washington, DC.

Fell, D. (1994). "Safety Update: Problem Definition and Countermeasure Summary: Fatigue." New South Wales Road Safety Bureau, RUS No. 5.

Fujioka, T., Shirano, Y., and Matsushita, A. "Driver's Behavior Under Steering Assist Control System." IEEE Conference on Intelligent Transportation Systems, Tokyo, Japan, 246-251.

Fukuda, J., Akutsu, E., and Aoki, K. (1995). "Estimation of Driver's Drowsiness Level Using Interval of Steering Adjustment for Lane Keeping." JSAE Review, Society of Automotive Engineers of Japan, 16(2), 197-199.

Gander, P., and James, I. (1999). "Investigating Fatigue in Truck Crashes.” Wellington School of Medicine and Commercial Vehicle Investigation, New Zealand.

Glaser, R. A., and Fisher, D. L. "Driving simulators: Are they valid test environments for ITS development?" Proceedings of the Human Factors and Ergonomics Society 41st Annual Meeting, Albuquerque, NM.

Godthelp, J. (1985). "Precognitive Control: Open and Closed Loop Steering in a Lane Change Manoeuvre." Ergonomics, 28, 1419-1438.

Graham, R., and Mitchell, V. A. "An Experimental Study into the Ability of Drivers To Assimilate and Retain In-Vehicle Traffic Information." Vehicle Navigation and Information Systems Conference, Yokohama, Japan.

Green, P., Levison, W., Paelke, G., and Serafin, C. (1995). "Preliminary Human Factors Design Guidelines for Driver Information Systems." Federal Highway Administration, Publication No. FHWA-RD-94-087, Washington, DC.

Gruening, J., Bernard, J., Clover, C., and Hoffmeister, K. (1998). "Driving Simulation.” SAE Special Publications: Vehicle Dynamics and Simulation, 1361(980223), 4959.

Gupta, N., Bisantz, A. M., and Singh, T. (2002). "The Effects of Adverse Condition Warning System Characteristics on Driver Performance: An Investigation of Alarm Signal Type and Threshold Level." Behavior and Information Technology, 21(4), 235-248. 
Haider, E., and Rohmert, W. (1976). "Blink Frequency during Four Hours of Simulated Truck Driving." European Journal of Applied Psychology, 35, 137-147.

Hamouda, G., and Saccomono, F. "Neural Network Model for Truck Driver Fatigue Accident Detection." Canadian Conference on Electrical and Computer Engineering, Montreal, Canada, 362-365.

Harris, W., and et al (1972). "A Study of the Relationships Among Fatigue, Hours of Service, and Safety of Operations of Truck and Bus Drivers." U.S. Department of Transportation BMCS-RD-71-2, Washington, DC.

Haworth, N. L., and Vulcan, P. "Testing of Commercially-Available Fatigue Monitors." Le Maintien de la Vigilance dans les Transports, Transports et Communication, Caen: Pardigme.

Haykin, S. (1994). Neural Networks: A Comprehensive Foundation, Prentice Hall, Englewood Cliffs, NJ.

Hertz, R. P. (1998). “Tractor-Trailer Driver Fatality: The Role of Non-Connective Rest in a Sleeper Berth." Accident Analysis and Prevention, 20(6), 429-431.

Hirst, S., and Graham, R. (1997). "The Format and Presentation of Collision Warnings." Ergonomics and Safety Intelligent Driver Interfaces, Y. I. Noy, ed., NJ, 203-219.

Hogema, J. H. (1997). “Compensation for Delay in the Visual Display of a Driving Simulator.” Simulation, 69(1), 27-34.

Horne, J. A., and Reyner, L. A. (1995). "Sleep related vehicle accidents." British Medical Journal, 310(6979), 565-567.

Horowitz, A. D., and Dingus, T. D. "Warning Signal Design: A Key Human Factors Issue in an In-vehicle Front-to-rear-end Collision Warning System." Proceedings of the Human Factors Society 36th Annual Meeting, Atlanta, GA.

Hoshino, H., Kojima, S., Uchiyama, Y., and Hongo, T. (2002). "Evaluation of Effects on Improvement in a Driver's reaction by Spatial Warning Sounds." IEICE Transactions on Information and Systems, E85-D(11), 1793-1800.

Howe, J. G., Rupp, M. Y., Jang, B.-C., Woodburn, C. M., Guenther, D. A., and Heydinger, G. J. (1997). "Improving Steering Feel for the National Advanced Driving Simulator." SAE Special Publications: Research into Vehicle Dynamics and Simulation, 1228(970567), 135-145.

Huang, R. S., Kuo, C. J., Tsai, L. L., and Chen, O. "EEG Pattern Recognition - Arousal States Detection and Classification." IEEE International Conference on Neural Networks, Washington, DC.

Hulbert, S. (1972). “Effects of Driver Fatigue.” Human Factors in Highway Traffic Safety Research, T. W. Forbes, ed., Wiley and Sons, NY. 
Isomoto, K., Niibe, T., Suetomi, T., and Butsuen, T. "Development of a Lane-keeping System for Lane Departure Avoidance." 2nd World Congress on ITS, Yokohama, Japan.

Itoh, M., Abe, G., and Tanaka, K. "Trust in and Use of Automation: Their Dependence on Occurrence Patterns of Malfunctions." IEEE International Conference on System, Man, and Cybernetics, Tokyo, Japan, 715-720.

Jang, B. C. (1996). “A Mathematical Model of a Power Steering System for Implementation in a Driving Simulator," The Ohio State University.

Janssen, W., and Nilsson, L. (1993). “Behavioral Effects of Driver Support.” Driving Future Vehicle, A. M. Parkes and S. Fransen, eds., Taylor and Francis, London, England, 147-155.

Janssen, W., and Thomas, H. "In-Vehicle Collision Avoidance Support under Adverse Visibility Conditions." Proceedings of the International Ergonomics Association, Toronto, Canada, 179-181.

Jovanis, P., Kaneko, T., and Lin, T. "Exploratory Analysis of Motor Carrier Accident Risk and Daily Driving Patterns." 70th Annual Meeting of Transportation Research Board, Washington, DC.

Kahneman, D. (1973). Attention and Effort, Prentice Hall, Englewood Cliffs, NJ.

Kiefer, R. J. "Developing a Forward Collision Warning System Timing and Interface Approach by Placing Drivers in Realistic Rear-end Crash Situations." IEA 2000/HFES 2000 Congress, San Diego, CA.

Kim, J.-Y., Park, M.-Y., and Park, C.-S. "Psycho-physiological Responses Reflecting Driver's Emotional Reaction to Interior Noise during Simulated Driving." Proceedings of the XIVth Triennial Congress of the International Ergonomics Association and 44th Annual Meeting of the Human Factors and Ergonomics Association, 'Ergonomics for the New Millennium', San Diego, CA.

Lavie, P. (1986). 'Ultrashort sleep-waking schedule, III. 'Gates' and 'Forbidden Zones' for Sleep.” Electroencephalography and Clinical Neurophysiology, 63, 414-425.

Lee, G. C. H., Yoo, Y., and Jones, S. (1997). "Investigation of Driving Performance, Vection, Postural Sway, and Simulator Sickness in a Fixed-based Driving Simulator." Computers \& Industrial Engineering, 33(3-4), 533-536.

Lee, J., and Moray, N. (1992). "Trust, Control Strategy and Allocation of Function in Human-machine Systems.” Ergonomics, 35(10), 1243-1270.

Lee, J. D., Hoffman, J., and Hayes, E. "Collision Warning Design to Mitigate Driver Distraction." Proceedings of the SIGCHI conference on human factors in computing systems (CHI'04), Vienna, Austria. 
Lee, J. D., and Kantowitz, B. H. (1998). "Perceptual and Cognitive Aspects of Intelligent Transportation Systems." Human Factors in Intelligent Transportation Systems, W. Barfield and T. A. Dingus, eds., Lawrence Erlbaum Associates, Mahwah, NJ, 31-54.

Lee, J. D., McGehee, D. V., Brown, T. L., and Reyes, M. L. (2002). "Collision Warning Timing, Driver Distraction, and Driver Response to Imminent rear-End Collision in a High-Fidelity Driving Simulator." Human Factors, 44(2), 314.

Lee, M. D., and Patterson, R. W. (1993). "The Application of Three-dimensional Auditory Displays to Aircraft Cockpits: User requirements, Technology Assessments, and Operational Recommendations." Lockheed Corporation.

Lerner, N. D., Kotwal, B. M., Lyons, R. D., and et al (1996). "Preliminary Human Factors Guidelines for Crash Avoidance Warning Devices.” NHTSA report DOT 808 342, Washington, DC.

Lesch, M. F. (2003). "Comprehension and Memory for Warning Symbols: Age-related Differences and Impact of Training." Journal of Safety Research, 34.

Levine, O. H., and Mourant, R. R. "Effect of Visual Display Parameters on Driving Performance in a Virtual Environments Driving Simulator." Proceedings of the 40th Human Factors and Ergonomics Society, Philadelphia, PA, 1136-1140.

Lin, D., Jovanis, P., and Yang, C. (1994). "Time of Day Models of Motor Carrier Accident Risk." Transportation Research Record, 1457.

Lin, J. J.-W., Duh, H. B. L., Parker, D. E., Abi-Rached, H., and Furness, T. A. "Effects of Field of View on Presence, Enjoyment, Memory, and Simulator Sickness in a Virtual Environment." IEEE Virtual Reality Annual International Symposium, Orlando, FL, 164-171.

Liu, A., and Chang, S. "Force Feedback in a Stationary Driving Simulator." IEEE International Conference on Systems, Man and Cybernetics, Vancouver, Canada, 1711-1716.

Lloyd, M. M., Wilson, G. D., Nowak, C. J., and Bittner Jr., A. C. (1999). "Brake Pulsing as Haptic Warning for an Intersection Collision Avoidance Countermeasure." Transportation Research Record, 1694, 34-41.

Mackie, R., and Miller, C. (1978). "Effects of Hours of Service, Regularity of Schedules and Cargo Loading on Truck and Bus Driving Fatigue." Coleta, C, Technical Report No. 1765-F.

Mackie, R., and Wylie, C. D. "Countermeasures To Loss Of Alertness In Motor Vehicle Drivers: A Taxonomy And Evolution." Proceedings of the Human Factors Society 35th Annual Meeting, San Francisco, CA, 1149-1153.

Mackie, R. R., and O'Hanlon, J. F. (1977). "A Study of the Combined Effects of Extended Driving and Heat Stress on Driver Arousal and Performance." 
Vigilance: Theory, Operational Performance and Physiological Correlates, R. R. Mackie, ed., Plenum Press, NY.

Makeig, S., and Inlow, M. (1993). "Lapses in Alertness: Coherence of Fluctuations in Performance and EEG Spectrum." Electroencephalography and Clinical Neurophysiology, 86, 23-35.

Mast, T., Jones, H., and Heimstra, N. (1989). "Effects of Fatigue on Performance in a Driving Device. Highway Research Record.” Driver Fatigue Research: Development of Methodology, Haworth, Vulcan, Triggs, and Fildes, eds., Accident Research Center, Monash University Australia.

McBain, W. (1970). “Arousal, Monotony, and Accidents in Line Driving." Journal of Applied Psychology, 54, 509-519.

McCartt, A., Rohrbaugh, J., Hammer, M., and Fuller, S. (2000). "Factors Associated With Falling Asleep at the Wheel Among Long-Distance Truck Drivers." Accident Analysis and Prevention, 32, 493-504.

McCartt, T., Ribner, S., Pack, A., and Hammer, M. (1996). "The Scope and Nature of the Drowsy Driving Problem in the New York State." Accident Analysis and Prevention, 28, 511-517.

McGehee, D. V., Brown, T. L., Lee, J. D., and Wilson, T. B. (2002). "Effect of Warning Timing on Collision Avoidance Behavior in a Stationary Lead Vehicle Scenario." Transportation Research Record, 1803(02-3746), 1-7.

McGehee, D. V., Dingus, T. D., and Horowitz, A. D. “An Experimental Field Test of Automotive Headway Maintenance/Collision Warning Visual Displays." Proceedings of the Human Factors and Ergonomics Society 38th Annual Meeting, Nashville, TN.

Meredith, C., and Edworthy, J. (1995). "Are There Too Many Alarms in the IntensiveCare Unit: An Overview of the Problems." Journal of Advanced Nursing, 21(1), $15-20$.

Mitler, M., Miller, J., Lipsitz, J., Walsh, K., and Wylie, C. (1997). "The Sleep of LongHaul Truck Drivers.” New England Journal of Medicine, 337 (11), 755-761.

Miyamoto, T., Momiyama, F., and Fujioka, T. (1991). "Steer-restoring Torque Controlled Driving Simulator for Developing Steering Road Feel." SAE Special Publications: Commercial Vehicle Suspensions, Steering Systems, and Traction, 892, 83-88.

Mourant, R. R., and Thattacherry, T. R. "Simulator Sickness in a Virtual Environments Driving Simulator." The XIVth Triennial Congress of the International Ergonomics Association and 44th Annual Meeting of the Human Factors and Ergonomics Association, 'Ergonomics for the New Millennium', San Diego, CA, 534-537. 
Muir, B. M. (1994). "Trust in Automation: Part I. Theoretical Issues in the Study of Trust and Human Interaction in Automated Systems." Ergonomics, 37(11), 19051922.

Naab, K., and Reichart, G. "Driver Assistance Systems for Lateral and Longitudinal Vehicle Guidance: Heading Control and Active Cruise Support." AVEC '94 Symposium, Tsukuba, Japan, 449-454.

Naitoh, P. (1992). "Minimal Sleep to Maintain Performance: the Search for Sleep Quantum in Sustained Operations." Why We Nap: Evolution, Chronobiology, and Functions of Polyphasic and Ultrashort Sleep, C. Stampi, ed., Birkhauser, Boston, MA, 199-216.

Nilsson, L. "Behavioral Research in an Advanced Driving Simulator: Experiences of the VTI System." Proceedings of the Human Factors and Ergonomics Society, Seattle, WA.

O'Hanlon, J. F. (1978). "What is the Extent of the Driving Fatigue Problem?" Driving Fatigue in Road Traffic Accidents, Commission of the European Communities Report No.EUR6065EN, 19-25, Brussels, Belgium.

Ogawa, K., and Shimotani, M. (1997). "Drowsiness Detection System." Mitsubishi Electric Advance, 78, 13-16.

Onken, R. "DAISY: An Adaptive, Knowledge-Based Driver Monitoring and Warning System.'" IEEE Vehicle Navigation and Information Conference, Yokohama, Japan.

Pack, A. L., Pack, A. M., Rodgman, E., Cucchiara, A., Dinges, D. F., and Schwab, C. W. (1995). "Characteristics of Crashes Attributed to the Driver Having Fallen Asleep." Accident Analysis and Prevention, 27(6), 769-775.

Parasuraman, R., Hancock, P. A., and Olofinboba, O. (1997). “Alarm Effectiveness in Driver-Centred Collision Warning Systems.” Ergonomics, 40(3), 390-399.

Payandeh, S., and Stanisic, Z. "On Application of Virtual Fixtures as an Aid for Telemanipulation and Traning.” IEEE VR2002, Haptics Symposium, Orlando, FL.

Pilutti, T., and Ulsoy, G. "Identification of Driver State for Lane-Keeping Tasks: Experimental Results." The American Control Conference, Seattle, WA, 16671671.

Reymond, G., Kemeny, A., Droulez, J., and Berthoz, A. (2001). "Role of Lateral Acceleration in Curve Driving: Driver Model and Experiments on a Real Vehicle and a Driving Simulator." Human Factors, 43(3), 483-495.

Riemersama, J. B., Sanders, A. F., Wildervack, C., and Gaillard, A. W. (1977).

"Performance Decrement During Prolonged Night Driving." Vigilance: Theory, Operational Performance and Physiological Correlates, R. R. Makie, ed., Plenum Press, NY. 
Ross, T., Mitland, K., Fuchs, M., Pauzie, A., Engert, A., Duncan, B., Vaughan, G., Vernet, M., Peters, H., Burnett, G., and May, A. (1996). "HARDIE Design Guidelines Handbook: Human Factors Guidelines for Information Presentation." ATT Systems, Commission of the European Communities, Luxembourg.

Ryder, J., Malin, S., and Kinsley, C. (1981). "The Effects of Fatigue and Alcohol on Highway Safety.” NHTSA Report No. DOT-HS-805-854, Washington, DC.

Safford, R., and Rockwell, T. H. (1967). "Performance Decrement in Twenty Four Hour Driving." Highway Research Record, 163, 68-79.

Sagberg, F. (1999). "Road Accidents Caused by Drivers Falling Asleep.” Accident Analysis and Prevention, 31, 639-649.

Sayed, R., and Eskandarian, A. (2001). "Unobtrusive Drowsiness Detection by Neural Network Learning of Driver Steering." Journal of Automobile Engineering, 215(D9), 969-975.

Seki, M., Shimotani, M., and Nishida, M. (1998). "Study of Blink Detection Using Bright Pupils.” JSAE Review, Society of Automotive Engineers of Japan, 19(1), 58-60.

Seko, Y. (1984). "Present Technological Status of Detecting Drowsy Driving Patterns." Jidosha Gijutsu, 30(5).

Shafer, J. H. "The Decline of Fatigue Related Accidents on NYS Thruway.” Proceedings of the Highway Safety Forum on Fatigue, Sleep Disorders and Traffic Safety, Albany, NY.

Siegmund, G. P., King, D. J., and Mumford, D. K. (1996). "Correlation of Steering Behavior with Heavy-truck Driver Fatigue." SAE Special Publications, 1190, 1738.

Skipper, J. H., Wierwille, W., and Hardee, L. (1984). "An Investigation of Low Level Stimulus Induced Measures of Driver Drowsiness." Virginia Polytechnic Institute and State University IEOR Department Report \#8402, Blacksburg, VA.

Steele, M., and Gillespie, B. "Shared Control between Human and Machine." Proceedings of the 45th Annual Meeting of the Human Factors and Ergonomics Society, Minneapolis, MN.

Stein, A. C. (1995). "Detecting Fatigued Drivers with Vehicle Simulators.” Driver Impairment, Driver Fatigue and Driving Simulation, L. Hartley, ed., Taylor \& Francis, Bristol, PA, 133-150.

Stoohs, R., Guilleminault, C., and Dement, W. (1993). "Sleep Apnea and Hypertension in Commercial Truck Drivers.” Sleep, 16, S11-S14.

Stutts, J., Wilkins, J., S., O., and Vaughn, B. (2003). "Driver Risk Factors for SleepRelated Crashes." Accident Analysis and Prevention, 35, 321-331. 
Sudarsan, S. P., Cohen, C. J., Du, L. Q., Cobb, P. N., Yager, E. S., and Jacobus, C. J. "Influence of Video Characteristics of Simulator Images on Remote Driving Performance." IEEE International Conference on Systems, Man and Cybernetics, Orlando, FL, 1062-1066.

Suzuki, K., and Jansson, H. (2003). “An Analysis of Driver's Steering Behavior During Auditory or Haptic Warnings for the Designing of Lane Departure Warning System." JSAE Review, Society of Automotive Engineers of Japan, 24, 65-70.

Sweeney, M., Ellingstad, V., Mayer, D., Eastwood, M., Weinstein, E., and Loeb, B. "The Need for Sleep: Discriminating between Fatigue-related and Non-Fatigue Related Truck Accidents." The Human Factors and Ergonomics Society Annual Meeting, San Diego, CA, 1122-1126.

Tepas, D. I., and Paley, M. J. "Managing Alertness and Fatigue in Advanced IVHS." The IVHS America Annual Meeting, Washington, DC, 785-789.

Thiffault, P., and Bergeron, J. (2003a). "Monotony of Road Environment and Driver Fatigue: A Simulator Study." Accident Analysis and Prevention, 35.

Thiffault, P., and Bergeron, J. (2003b). "Fatigue and Individual Differences in Monotonous Simulated Driving." Personality and Individual Differences, 34, 159-176.

Tilley, D. H., Erwin, C. W., and Gianturco, D. T. "Drowsiness and Driving: Preliminary Report of a Population Survey." Society of Automotive Engineers International Automotive Engineering Congress, Detroit, MI.

Torsvall, L., and Akerstedt, T. (1987). "Sleepiness on the Job: Continuously Measured EEG in Train Drivers.” Electroencephalography and Clinical Neurophysiology, $66,502-511$.

U.S. NTSB (1990). "Fatigue, Alcohol, Other Drugs, and Medical Factors in Fatal-to-theDriver Heavy Truck Crashes, Volume 1.” NTSB Safety Study NTSB/SS-90-01, Washington, DC.

Uaneras, R. E., Green, C. A., Kiefer, R. J., Chundnik, W. J., Altan, O. D., and Singer, J. P. (2005). "Design and Evaluation of a Prototype Rear Obstacle Detection and Driver Warning System.” Human Factors, 47(1), 199-215.

Ueno, H., Kaneda, M., and Tsukino, M. "Development of Drowsiness Detection System." IEEE Vehicle Navigation and Information Systems Conference, Yokohama, Japan, 15-20.

Vallet, M., Fakhar, S., Olivier, D., and Baez, D. "Detection and Control of the Degree of Vigilance of Drivers." 13th International Conference on Experimental Safety Vehicles, Paris, France. 
Verwey, W. B., and Zaidel, D. M. (2000). "Predicting Drowsiness Accidents from Personal Attributes, Eye Blinks and Ongoing Driving Behaviour." Personality and Individual Differences, 28, 123-142.

Wang, J. S., and Knipling, R. R. (1994). "Single Vehicle Roadway Departure Crashes: Problem Size Assessment and Statistical Description." U.S. Department of Transportation, National Highway Traffic Safety Administration, Washington, DC.

Weber, J. W., Mullins, C. A., Schumacher, P. W., and Wright, C. D. “A Systems Approach to the Development of an Integrated Collision Avoidance Vehicle." The Vehicle Navigation and Information Systems Conference, Yokohama, Japan, 431434.

Wierwille, W. W., and Ellsworth, L. A. (1994). "Evaluation of Driver Drowsiness by Trained Raters." Accident Analysis and Prevention, 26(5), 571.

Wierwille, W. W., Ellsworth, L. A., Wreggit, S. S., Fairbanks, R. J., and Kirn, C. L. (1994). "Research on Vehicle-Based Driver Status/Performance Monitoring: Development, Validation, and Refinement of Algorithms for Detection of Driver Drowsiness." NHTSA Final Report: DOT HS 808 247, 1994, Washington, DC.

Wierwille, W. W., Wreggit, S. S., and Mitchell, M. W. (1992). "Research on Vehicle Based Driver Status/Performance Monitoring. First Semi-Annual Research Report.” NHTSA Cooperative Agreement Number DTNH 22-91-Y-07266, Washington, DC.

Wilkinson, T., Edwards, S., and Haines, E. (1966). "Performance Following a Night of Reduced Sleep." Psychonomic Science, 5, 471-472.

Wylie, C. D., Shultz, T., Miller, J. C., Mitler, M. M., and Makie, R. R. (1996). "Commercial Motor Vehicle Driver Fatigue and Alertness Study, Project Report." Federal Highway Administration, DOT Report No. FHWA-MC-97-002, Washington, DC.

Yabuta, K., Iizuka, H., Yanagishima, T., Kataoka, Y., and Seno, T. "The Development of Drowsiness Warning Devices." Proceedings of the 10th International technical Conference on Experimental Safety Vehicles, Washington, DC.

Young, T., Blustein, J., Finn, L., and Palta, M. (1997). "Sleep-Disordered Breathing and Motor Vehicle Accidents in a Population-Based Sample of Employed Adults." Sleep, 20(8), 608-613. 